\title{
SURFACE EFFECTS IN SEgMENTEd Silicon SENSORS
}

\author{
Dissertation \\ zur Erlangung des Doktorgrades \\ an der Fakultät für Mathematik, Informatik \\ und Naturwissenschaften
}

Fachbereich Physik

der Universität Hamburg

\author{
vorgelegt von \\ IOANNIS KOPSALIS \\ geboren am 17.08.1990 \\ aus Agrinio, Griechenland
}

Hamburg

2017 
Gutachter der Dissertation:

Mitglieder der Prüfungskommission:

Datum der Disputation: Vorsitzender des Prüfungskommission: Vorsitzender des Promotionsausschusses: Dekan der MIN Fakultät: Leiter des Department Physik:
Prof. Dr. ERIKA GARUTTI

Prof. DR. Robert KLANNER

PROF. DR. ERIKA GARUTTI

PROF. DR. ROBERT KLANNER

PROF. DR. HEINZ GRAAFSMA

PROF. DR. GÜNTER SIGL

DR. GEORG STEINBRÜCK

16.02.2017

PROF. DR. GÜNTER SIGL

Prof. DR. WOLFGANG HANSEN

Prof. Dr. HeINRICH GraEnER

Prof. DR. MichaEl PotTHOFF 


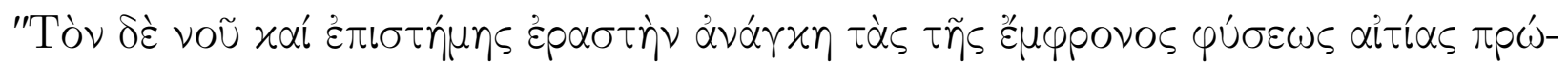

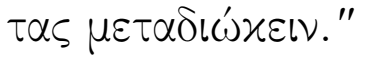

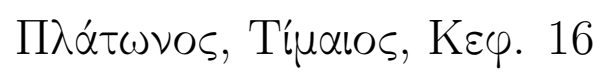

"The lover of reason and knowledge must first seek for the causes which belong to the rational order."

Plato, Timaeus, Ch. 16 
To my parents... 


\section{Abstract}

Silicon detectors in Photon Science and Particle Physics require silicon sensors with very demanding specifications. New accelerators like the European X-ray Free Electron Laser (EuXFEL) and the High Luminosity upgrade of the Large Hadron Collider (HL-LHC), pose new challenges for silicon sensors, especially with respect to radiation hardness. High radiation doses and fluences damage the silicon crystal and the $\mathrm{SiO}_{2}$ layers at the surface, thus changing the sensor properties and limiting their life time. Non-Ionizing Energy Loss (NIEL) of incident particles causes silicon crystal damage. Ionizing Energy Loss (IEL) of incident particles increases the densities of oxide charge and interface traps in the $\mathrm{SiO}_{2}$ and at the $\mathrm{Si}-\mathrm{SiO}_{2}$ interface.

In this thesis the surface radiation damage of the $\mathrm{Si}_{-} \mathrm{SiO}_{2}$ system on high-ohmic $\mathrm{Si}$ has been investigated using circular MOSFETs biased in accumulation and inversion at an electric field in the $\mathrm{SiO}_{2}$ of about $500 \mathrm{kV} / \mathrm{cm}$. The MOSFETs have been irradiated by X-rays from an X-ray tube to a dose of about $17 \mathrm{kGy}\left(\mathrm{SiO}_{2}\right)$ in different irradiation steps. Before and after each irradiation step, the gate voltage has been cycled from inversion to accumulation conditions and back. From the dependence of the drain-source current on gate voltage the threshold voltage of the MOSFET and the hole and electron mobility at the $\mathrm{Si}_{-} \mathrm{SiO}_{2}$ interface were determined. In addition, from the measured drain-source current the change of the oxide charge density during irradiation has been determined. The interface trap density and the oxide charge has been determined separately using the subthreshold current technique based on the Brews charge sheet model which has been applied for first time on MOSFETs built on high-ohmic Si. The results show a significant field-direction dependence of the surface radiation parameters. The extracted parameters and the acquired knowledge can be used to improve simulations of the surface radiation damage of silicon sensors. 



\section{Kurzfassung}

Siliziumdetektoren für die Forschung mit Photonen und für die Teilchenphysik benötigen Siliziumsensoren, die sehr hohe Anforderungen erfüllen müssen. Neue Beschleuniger, wie der European X-ray FreeElectron Laser (EuXFEL) und das High Luminosity-Upgrade des Large Hadron Collider (HL-LHC), stellen insbesondere im Hinblick auf Strahlungshärte neue Herausforderungen für Siliziumsensoren dar. Hohe Strahlungsdosen und -flüsse schädigen den Siliziumkristall sowie die $\mathrm{SiO}_{2}$-Schichten an der Oberfläche, wodurch sich die Sensoreigenschaften ändern und ihre Lebensdauer beschränkt wird. Nicht-ionisierender Energieverlust (NIEL) der einfallenden Teilchen verursacht Schäden im Siliziumkristall. Ionisierender En-

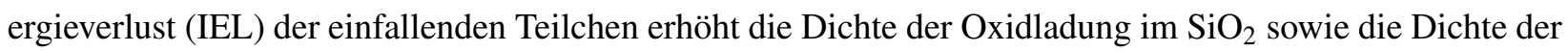
Grenzflächenhaftstellen am $\mathrm{Si}_{-} \mathrm{SiO}_{2}$ Übergang.

In dieser Arbeit wurde die Schädigung der Oberfläche des $\mathrm{Si}_{-} \mathrm{SiO}_{2}$-Systems von hochohmigem Si unter Verwendung kreisförmiger MOSFETs, die in Akkumulation und Inversion bei einem elektrischen Feld im $\mathrm{SiO}_{2}$ von etwa $500 \mathrm{kV} / \mathrm{cm}$ vorgespannt sind, untersucht. Die MOSFETs wurden mit Röntgenstrahlen aus einer Röntgenröhre bis zu einer Dosis von etwa $17 \mathrm{kGy}\left(\mathrm{SiO}_{2}\right)$ in verschiedenen Bestrahlungsschritten bestrahlt. Vor und nach jedem Bestrahlungsschritt wurde die Gate-Spannung von Inversion zu Akkumulation und zurück durchlaufen. Aus der Abhängigkeit des Drain-Source-Stroms von der Gate-Spannung wurden die Schwellenspannung des MOSFET sowie die Löcher- und Elektronenbeweglichkeit an der $\mathrm{Si}_{-} \mathrm{SiO}_{2}$ Grenzfläche bestimmt. Zusätzlich wurde aus dem gemessenen Drain-Source-Strom die Änderung der Oxidladungsdichte während der Bestrahlung bestimmt. Die Dichte der Grenzflächenhaftstellen und die Dichte der Oxidladung wurden separat bestimmt. Hierzu wurde die subthreshold current Technik, basierend auf dem Brewsschen charge sheet Modell, zum ersten Mal auf MOSFETs angewandt, die aus hochohmigem Si hergestellt wurden. Die Ergebnisse zeigen eine signifikante Feldrichtungsabhängigkeit der Parameter der Oberflächenstrahlungsschädigung. Die gewonnenen Parameter und das erworbene Wissen können zur Verbesserung der Simulation der Oberflächenstrahlungsschädigung von Siliziumsensoren verwendet werden. 



\section{Contents}

$\begin{array}{ll}\text { Abstract } & \text { v } \\ \text { v }\end{array}$

$\begin{array}{lll}\text { Kurzfassung } & \text { vii }\end{array}$

Contents $\quad$ ix

1 Introduction $\quad 1$

1.1 The sensor design projects: AGIPD and CMS Phase II pixel upgrade . . . . . . . . . . . . . 1

1.1.1 The AGIPD silicon pixel sensor . . . . . . . . . . . . . . . . . . 1

1.1.2 The CMS Phase II pixel upgrade . . . . . . . . . . . . . . . . . . . 3

1.2 Surface effects in segmented silicon sensors . . . . . . . . . . . . . . . . . . . 4

1.2.1 Surface parameters and TCAD simulations . . . . . . . . . . . . . . 6

1.2.2 Impact of surface effects on sensors . . . . . . . . . . . . . . . . . . . 7

1.3 Motivation and structure of this thesis . . . . . . . . . . . . . . . . 9

2 Physics of basic semiconductor and device theory 11

2.1 Metal Oxide Semiconductor MOS structures . . . . . . . . . . . . . . . . . . . . 11

2.1 .1 MOS Capacitor . . . . . . . . . . . . . . . . . . . 11

2.1.2 MOS Field Effect Transistor MOSFET . . . . . . . . . . . . . . . . . . . . 20

3 MOSFET structures and operation 3

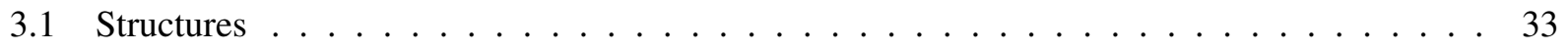

3.1.1 Drain-source current characteristics . . . . . . . . . . . . . . . . . 34

3.2 Length and width definitions . . . . . . . . . . . . . . . . . . . . . . 35

3.2.1 Series resistance and channel length . . . . . . . . . . . . . . . . . 35

3.3 Summary ............................... . . . . . . . . . . . . .

4 Basic mechanisms of radiation effects and the $\mathrm{Si}-\mathrm{SiO}_{2}$ system 41

4.1 Fundamentals of radiation environments and sources . . . . . . . . . . . . . . . . 41

4.1 Radiation sources . . . . . . . . . . . . . . . . . . . 41

4.1.2 Interaction of radiation with matter . . . . . . . . . . . . . . 41

4.2 Properties of $\mathrm{SiO}_{2}$ and the $\mathrm{Si}_{-} \mathrm{SiO}_{2}$ interface . . . . . . . . . . . . . . . . . 46

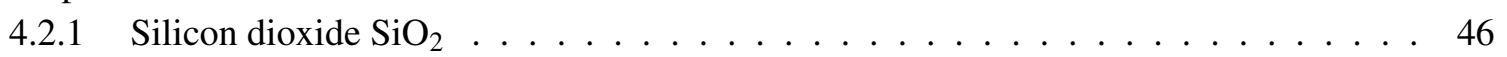

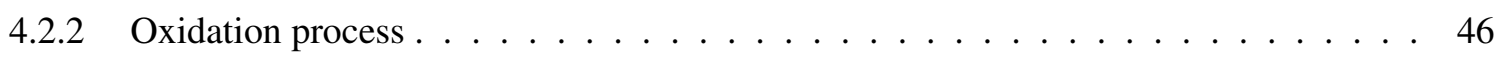

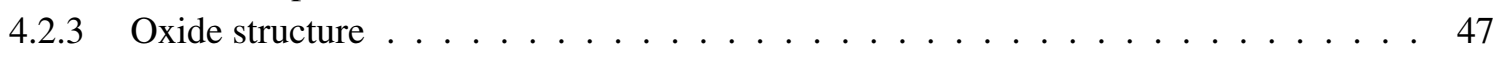

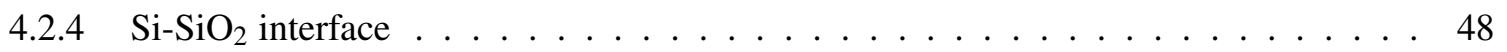

4.2.5 Nomenclature of oxide charges . . . . . . . . . . . . . . . . 50

4.3 Mechanisms of the build-up of radiation-induced surface damage . . . . . . . . . . . . 52

4.3.1 Charge generation and recombination $\mathrm{in}_{\mathrm{SiO}_{2}} \ldots \ldots \ldots \ldots 5$ 
4.3.2 Hole transport in $\mathrm{SiO}_{2} \ldots \ldots \ldots \ldots \ldots \ldots \ldots \ldots$

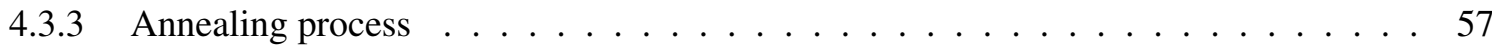

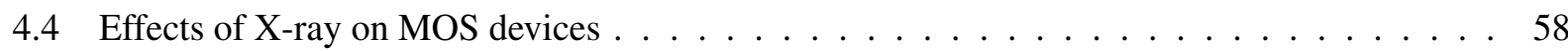

4.4.1 Radiation-Induced Charge Neutralization-RICN effect . . . . . . . . . . . . . . 58

4.4 .2 Rebound effect . . . . . . . . . . . . . . . . . . . 59

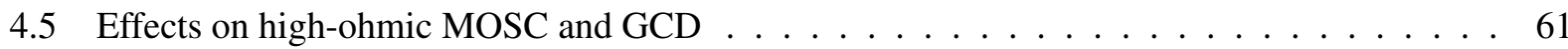

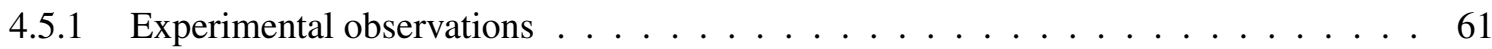

4.5.2 Electric field dependence of $N_{o x}^{e f f}$ on high-ohmic MOSC . . . . . . . . . . 63

$5 \quad$ X-ray dose and $E_{\text {field }}$ dependence of $N_{\text {ox }}^{\text {eff }} \quad \mathbf{6 7}$

5.1 High-ohmic p- and n-MOSFETs irradiated with $E_{\text {field }} \ldots \ldots \ldots$. . . . . . . . 67

5.1.1 The p-MOSFET irradiated with $E_{\text {field }}$ pointing from the $\mathrm{Si}$ into the $\mathrm{SiO}_{2} \ldots \ldots$. . . 67

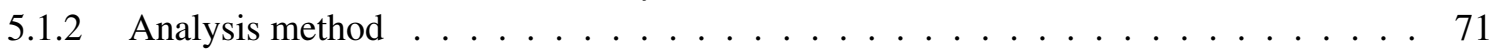

5.1.3 Effective oxide charge density $N_{o x}^{e f f} \ldots \ldots \ldots \ldots \ldots \ldots \ldots$. . . . . . . . . . . 74

5.1.4 The p-MOSFET irradiated with $E_{\text {field }}$ pointing from the $\mathrm{SiO}_{2}$ into the $\mathrm{Si} \quad \ldots \ldots$

5.1.5 The n-MOSFET irradiated with $E_{\text {field }}$ pointing from the $\mathrm{Si}$ into the $\mathrm{SiO}_{2} \quad \ldots \ldots$. . . 82

5.1.6 The n-MOSFET irradiated with $E_{\text {field }}$ pointing from the $\mathrm{SiO}_{2}$ into the $\mathrm{Si} \ldots \ldots 7$

5.1 .7 Comparison . . . . . . . . . . . . . . . . . . . . 95

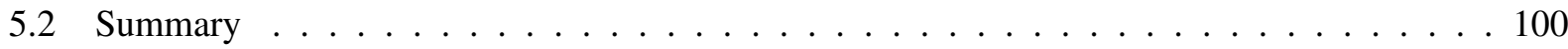

6 Subthreshold current technique $\quad 101$

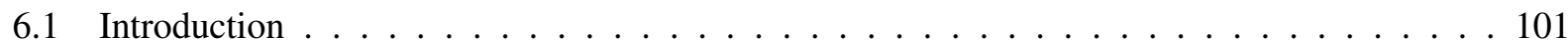

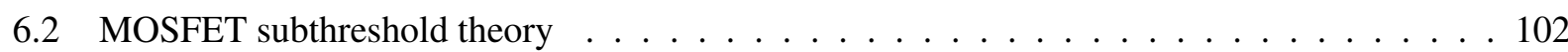

6.2.1 Theoretical MOSFET weak inversion channel current . . . . . . . . . . . . . . . . 102

6.2.2 Subthreshold charge separation technique . . . . . . . . . . . . . . . . 103

6.3 Discussion of variables and assumptions in the subthreshold charge separation technique . . 106

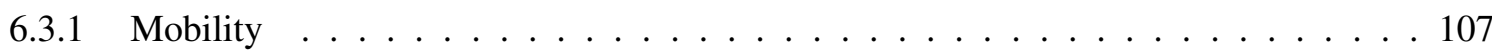

6.3 .2 Doping . . . . . . . . . . . . . . . . . . . . . . 109

6.3.3 Interface and trapped charge assumptions . . . . . . . . . . . . . . . . . . . . . . . . . . . . . . . . . .

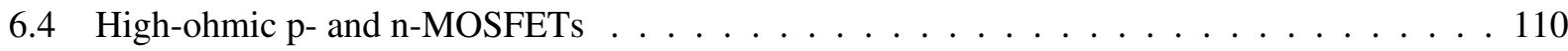

6.4.1 The p-MOSFET irradiated with $E_{\text {field }}$ pointing from the $\mathrm{Si}$ into the $\mathrm{SiO}_{2} \ldots \ldots \ldots$

6.4.2 The p-MOSFET irradiated with $E_{\text {field }}$ pointing from the $\mathrm{SiO}_{2}$ into the $\mathrm{Si} \ldots \ldots$

6.4.3 The n-MOSFET irradiated with $E_{\text {field }}$ pointing from the $\mathrm{Si}$ into the $\mathrm{SiO}_{2} \ldots \ldots \ldots$

6.4.4 The n-MOSFET irradiated with $E_{\text {field }}$ pointing from the $\mathrm{SiO}_{2}$ into the $\mathrm{Si} \ldots \ldots$

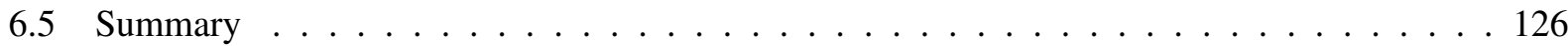

7 Gate Controlled Diode measurements on MOSFETs 127

7.1 Surface current . . . . . . . . . . . . . . . . . . . . . . . . 127

7.1.1 Before X-ray irradiation . . . . . . . . . . . . . . . . . . . . . 129

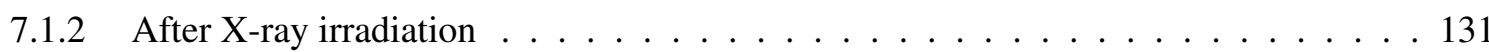

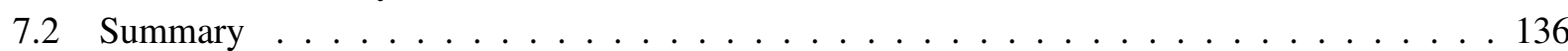

$8 \quad$ Annealing study of oxide charge and interface traps 137

8.1 The p-MOSFET irradiated with $E_{\text {field }}$ pointing from the $\mathrm{Si}$ into the $\mathrm{SiO}_{2} \ldots \ldots \ldots$. . . . 137

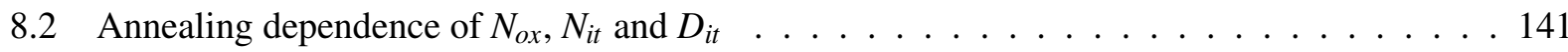

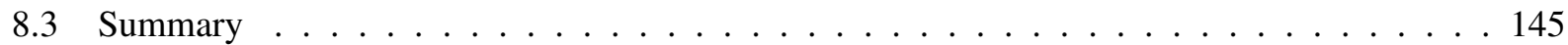

9 Critical evaluation of the subthreshold current technique 147

9.1 Evaluation for different drain-source voltage and ramp rate . . . . . . . . . . . . . . 147 
9.2 Evaluation of midgap and subthreshold swing method . . . . . . . . . . . . . . 151

9.2.1 The effect of charge lateral nonuniformities . . . . . . . . . . . . . 151

9.2.2 Comparison between midgap and subthreshold swing method . . . . . . . . . 152

9.3 Surface current contribution on the subthreshold curve . . . . . . . . . . . . . . . . 153

$\begin{array}{ll}10 \text { Summary and conclusions } & 157\end{array}$

$\begin{array}{ll}\text { Bibliography } & \text { i }\end{array}$

$\begin{array}{ll}\text { List of publications } & \text { xv }\end{array}$

$\begin{array}{ll}\text { Acknowledgements } & \text { xvii }\end{array}$

Declaration of Authorship $\quad$ xix 



\section{Introduction}

\subsection{The sensor design projects: AGIPD and CMS Phase II pixel upgrade}

\subsubsection{The AGIPD silicon pixel sensor}

The European X-ray Free-Electron Laser (EuXFEL) [1], currently being constructed at DESY, Hamburg and planned to be operational for users in 2017 , will deliver 27,000 fully coherent, high brilliance X-ray pulses per second with duration less than $100 \mathrm{fs}$ and time separation of $220 \mathrm{~ns}$. The unique features of the $\mathrm{X}$-ray beam pose major challenges for detectors used at the EuXFEL for imaging experiments, as shown in Fig. 1.1. A dynamic range of $0,1, \ldots$, up to more than $10^{4}$ photons per pulse, a frame rate of $4.5 \mathrm{MHz}$, and in particular a radiation tolerance of the senors for X-ray doses up to 1 GGy for 3 years of operation [2].

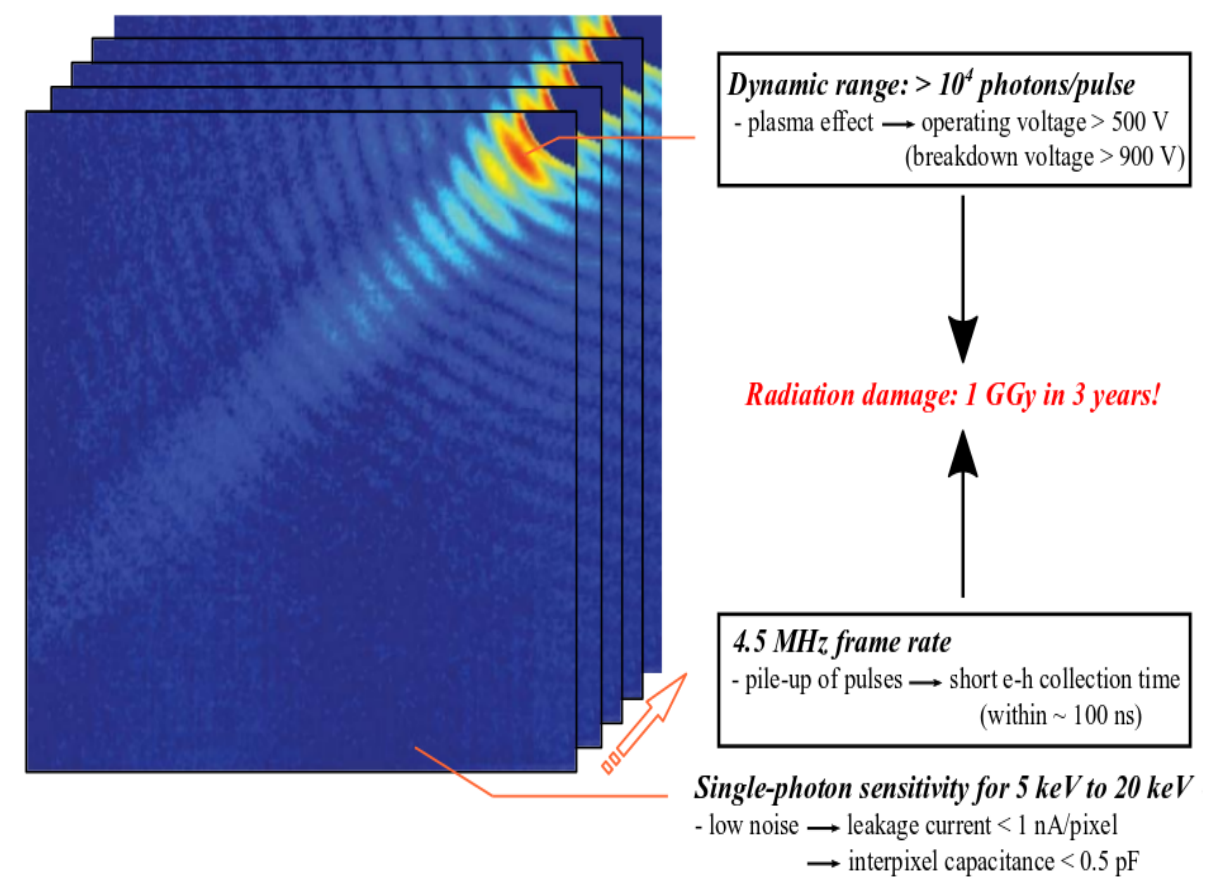

Figure 1.1: Challenges of silicon detectors used for imaging experiments at the EuXFEL. Figure adapted from $[3,4]$.

One of the detectors under development at the EuXFEL is the Adaptive Gain Integrating Pixel Detector (AGIPD) [5]. It is a hybrid detector system with 1 Mega Pixels, each of $200 \times 200 \mu \mathrm{m}^{2}$. The AGIPD detector consists of 16 modules, each with 16 ASICs bump-bonded to one $\mathrm{p}^{+} \mathrm{n}$ silicon pixel sensor. The 
sensor, Fig. 1.2, has been designed according to the optimized pixel and guard ring geometries and technological processes based on SYNOPSIS TCAD simulations $[4,6,7]$ with radiation damage related parameters implemented $[2,8]$.
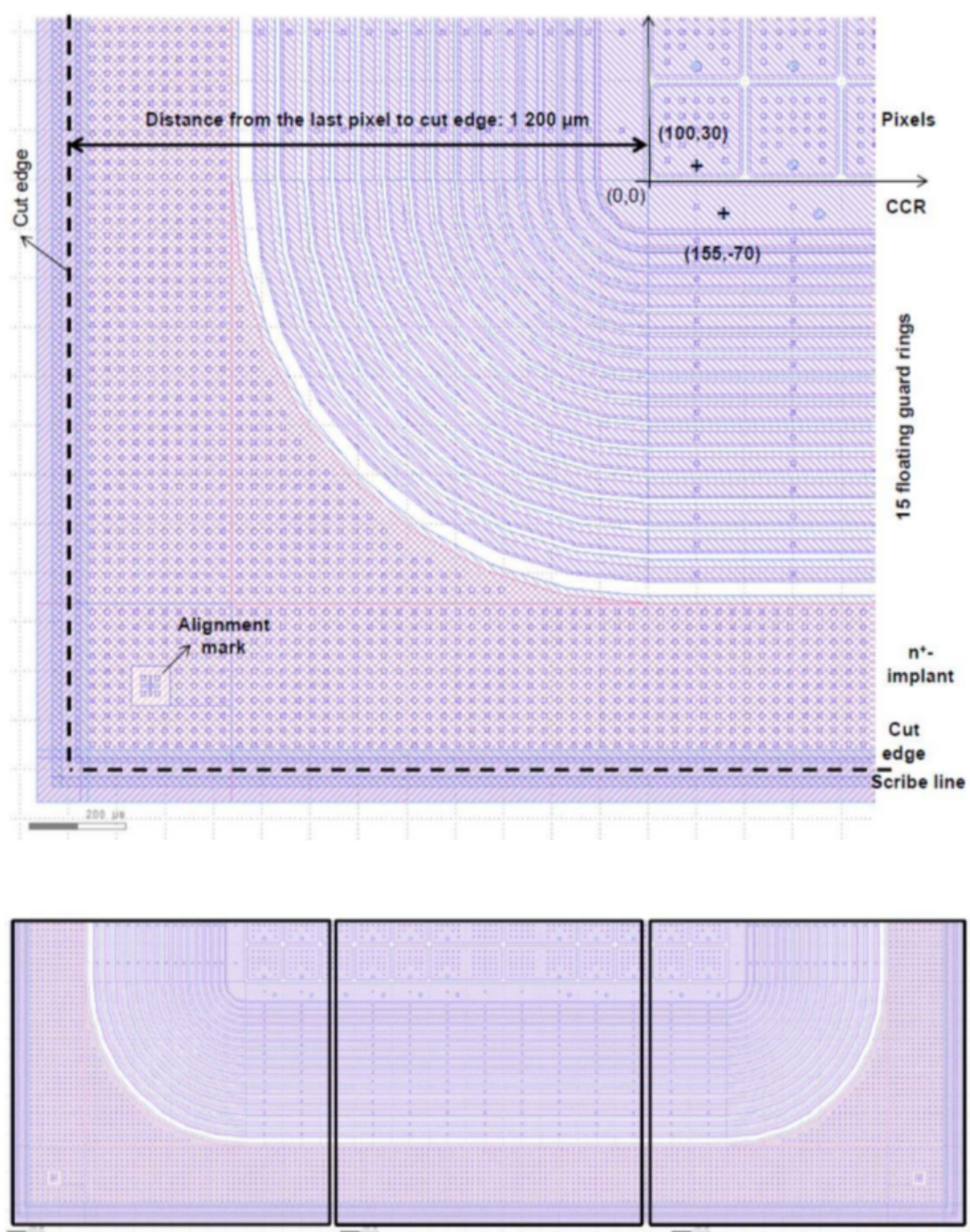

Figure 1.2: Layout and dimensions of the guard ring structure of the AGIPD sensor. Top: Layout and dimensions of the guard ring structure at the left lower corner of the sensor. Bottom: The guard ring structures at the left, middle and right lower corners of the sensor. Figure taken from $[4,8]$.

The sensors have been produced by SINTEF [9]. The basic properties of the silicon wafers have been obtained from electrical measurements on test structures. In order to demonstrate the radiation hardness of the AGIPD sensor, mini sensors with the same pixel and guard ring designs as the AGIPD and test structures have been irradiated up to $10 \mathrm{MGy}$ at the beamline P11 of PETRA III at DESY. From the test structures, the oxide charge density, $N_{o x}$, and the surface current density, $J_{\text {surf }}$, have been extracted as a function of X-ray dose. A saturation of $N_{o x}$ and $J_{\text {surf }}$ has been found between $100 \mathrm{kGy}$ and $1 \mathrm{MGy}$ and the saturation values are smaller than those from test structures produced by other vendors. From mini sensors, no breakdown has been found up to $900 \mathrm{~V}$ after irradiation and all electrical properties, in particular the leakage current and inter-pixel capacitance, are well within specifications [2]. 


\subsubsection{The CMS Phase II pixel upgrade}

The CMS experiment [10] intends to exchange the pixel detector for the high luminosity phase of the Large Hadron Collider (HL-LHC) at CERN. Therefore, a large R\&D effort has been started in order to develop silicon sensors capable of withstanding the expected high radiation damage. The targeted integrated luminosity of $3000 \mathrm{fb}^{-1}$, estimated for 10 years of operation at the design center-of-mass energy of $14 \mathrm{TeV}$, translates into an equivalent NIEL (Non-Ionizing Energy Loss) of $2 \cdot 10^{16} \mathrm{n}_{e q} \cdot \mathrm{cm}^{-2}$ and an IEL (Ionizing Energy Loss) dose in the $\mathrm{SiO}_{2}$ of $10 \mathrm{MGy}$ at the expected position of the innermost pixel detector layer [11]. The layout for the new CMS tracker, Fig. 1.3, is based on requirements to maintain efficient tracking under high luminosity conditions $[10,12,13]$. Outer tracker layers with modules consisting of two back-to-back strip sensors ( $2 \mathrm{~S}$ modules) are shown in red $(\mathrm{r}>60 \mathrm{~cm})$, layers with modules consisting of a macro pixel and a strip sensor (PS modules) are shown in blue $(20 \mathrm{~cm}<\mathrm{r}<60 \mathrm{~cm}$ ). The pixel detector, depicted in light blue and yellow, consists of four barrel layers $(\mathrm{r}<20 \mathrm{~cm})$ and 11 discs. The pixel Phase II barrel detector will cover approximately the same volume as the pixel Phase I detector.

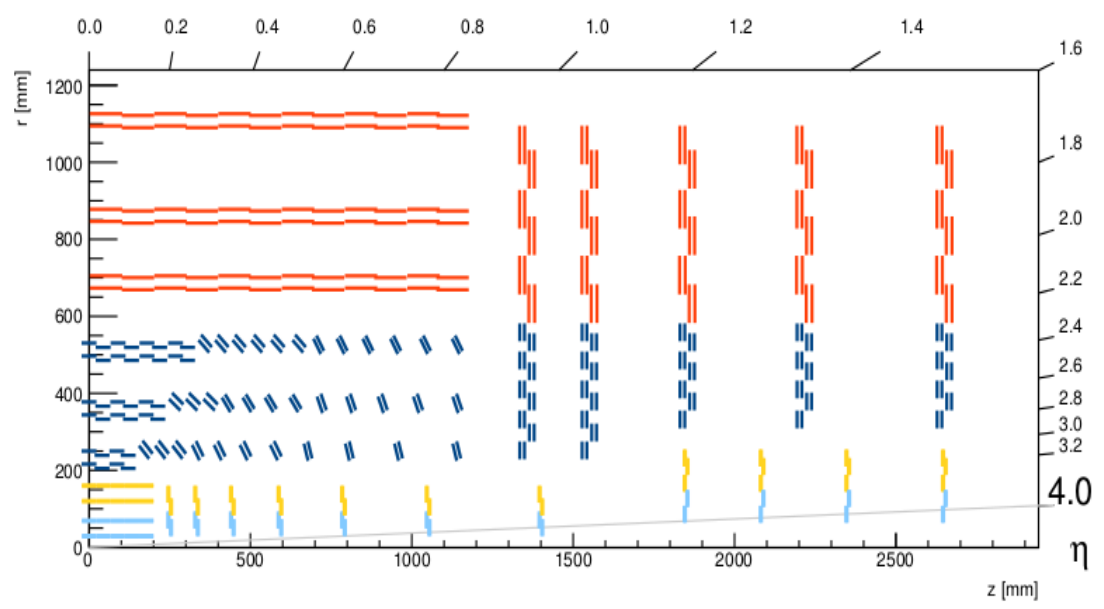

Figure 1.3: Layout of the CMS tracking system for the HL-LHC. Figure taken from [11].

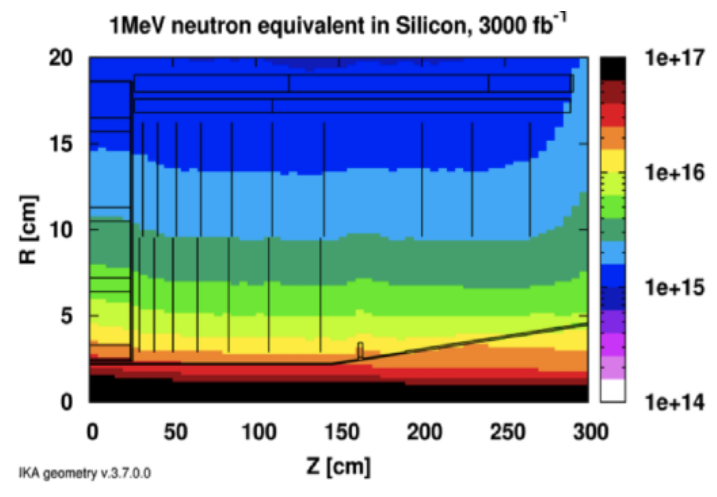

(a)

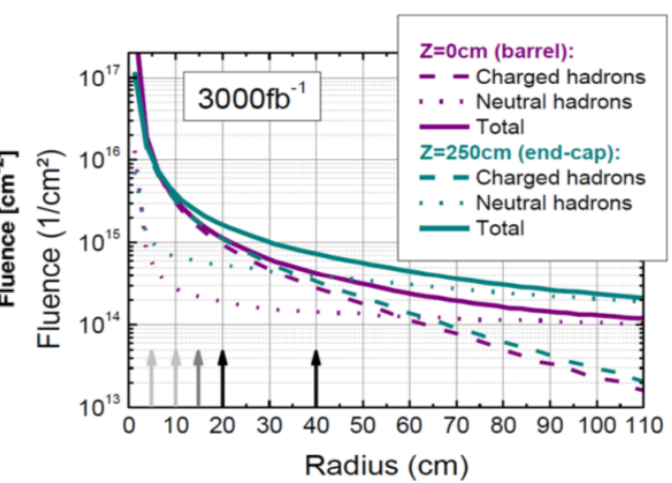

(b)

Figure 1.4: (a) Map of the expected particle fluence in the pixel volume, expressed in terms of $1 \mathrm{MeV}$ neutron equivalent $\left(\mathrm{n}_{e q}\right)$ fluence [10] and (b) Expected fluence in the CMS tracker [12] corresponding to an integrated luminosity of $3000 \mathrm{fb}^{-1}$. 
The expected particle fluence in the pixel volume, is presented in Fig. 1.4 (a). At the inner radius of the outer tracker, silicon modules are exposed to a mix of neutrons and charged particles (mainly pions) corresponding to $\mathrm{n}_{e q}$ fluences up to $1.5 \cdot 10^{15} \mathrm{~cm}^{-2}$, as shown in Fig. 1.4 (b). The corresponding ionizing dose at a radius of $20 \mathrm{~cm}$ is approximately $700 \mathrm{kGy}$. The operational properties of silicon sensors are strongly compromised at such fluences and the CMS Collaboration [10] performs studies to identify an optimal choice of sensor material and to optimize the sensor design $[12,14,15]$. In addition, due to the increased luminosity the cell size of future HL-LHC pixel sensors has to be downscaled to an area of about $2500 \mu \mathrm{m}^{2}$. Candidate pixel sizes under investigation are $25 \times 100 \mu \mathrm{m}^{2}$ and $50 \times 50 \mu \mathrm{m}^{2}$. Compared to the current CMS barrel pixel design, these dimensions allow little space for design choices and a biasing scheme like a punch through dot with a bias rail, shown in Fig. 1.5 (a). The CMS Collaboration has initiated a production of planar $\mathrm{n}^{+} \mathrm{p}$ pixel sensors on 6" wafers with a variety of different geometries, pixel isolation technologies (p-spray, p-stop), biasing schemes (common punch through: punch through biasing shared by four adjacent pixels) and bump bond patterns [13]. An example of the structures implemented is shown in Fig. 1.5 (b).

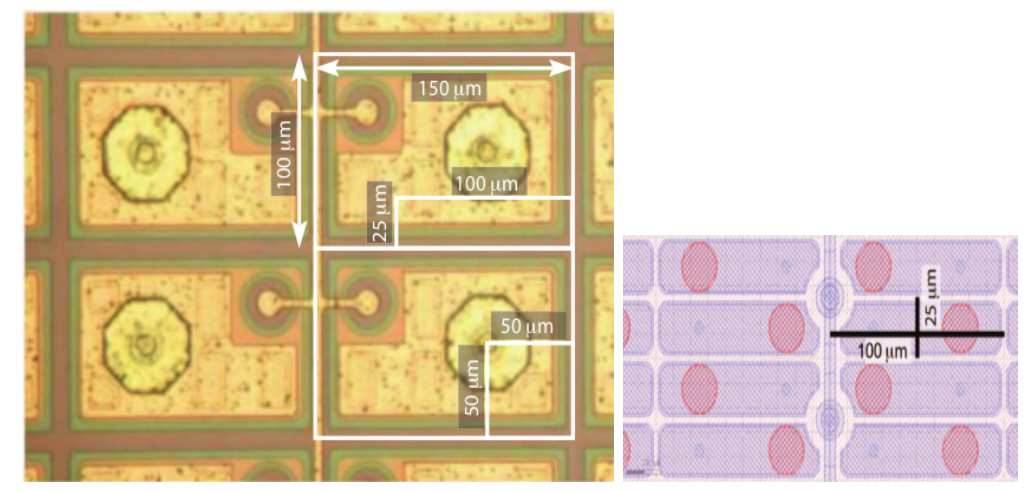

(a)

(b)

Figure 1.5: (a) Illustration of the required pixel dimensions of future CMS pixel sensors compared to the current CMS barrel pixel design and (b) Layout of $25 \times 100 \mu \mathrm{m}^{2}$ pixel cells with common punch through and p-spray pixel isolation. Figures taken from [13].

\subsection{Surface effects in segmented silicon sensors}

The voltage stability, charge collection properties and dark current of segmented silicon sensors are influenced by the charge and potential distributions on the sensor surface, the charge distribution in the oxide and passivation layers, and by $\mathrm{Si}_{-} \mathrm{SiO}_{2}$ interface states [16-21]. To better understand these phenomena, measurements on test structures and sensors before and after X-ray irradiation, and TCAD simulations including surface and interface effects are performed.

In particular the breakdown behavior, dark current and charge collection efficiency of segmented silicon sensors depends on the properties of the insulating layers and the electric boundary conditions on the sensor surface. These parameters can be measured and implemented in TCAD simulations [6]. Examples are given of the successful optimization of a pixel sensor for X-ray doses up to $1 \mathrm{GGy}$ [4] and of the explanation of the dose and humidity dependent charge collection efficiency observed for a silicon strip sensor [22-24]. They 
demonstrate that TCAD simulations with realistic parameters can explain surprising observations and avoid design mistakes.

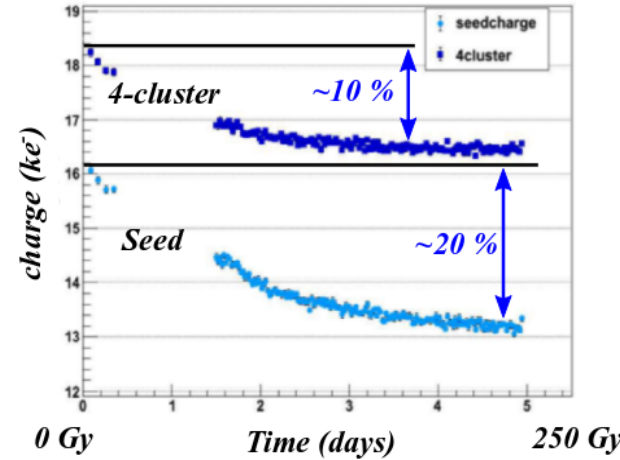

(a)

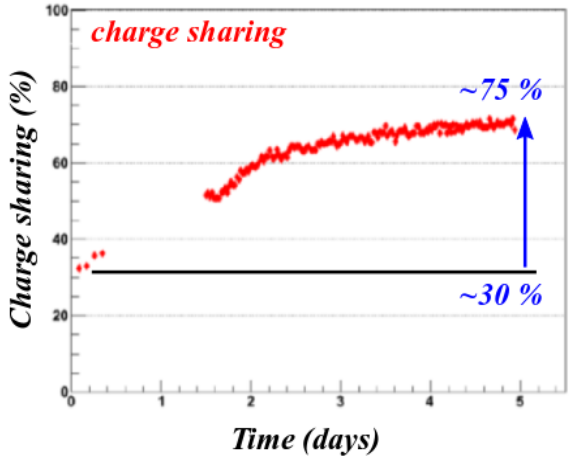

(b)

Figure 1.6: (a) Median of the $\mathrm{PH}(4-$ cluster) and $\mathrm{PH}($ seed) distributions as a function of time and (b) The corresponding time dependence for charge sharing. Figures taken from [14, 25].

An example from Refs. [14, 25], Fig. 1.6 (a) shows the time dependence of the median values of the pulseheight distributions of the 4-strip clusters, $\mathrm{PH}$ (4-cluster), and the corresponding pulse-height distributions for the seed clusters, PH(seed). Fig. 1.6 (b) shows the charge sharing. The measured data are referred to non-hadron irradiated p-spray strip sensor built on Float-Zone silicon, biased at $600 \mathrm{~V}$ during irradiation with a $\beta$ source. The initial dose is $0 \mathrm{~Gy}$ and the dose after 5 days, $250 \mathrm{~Gy}$. In order to explain the above observation, TCAD simulations were performed to calculate the electric field in the sensor. Fig. 1.7, shows

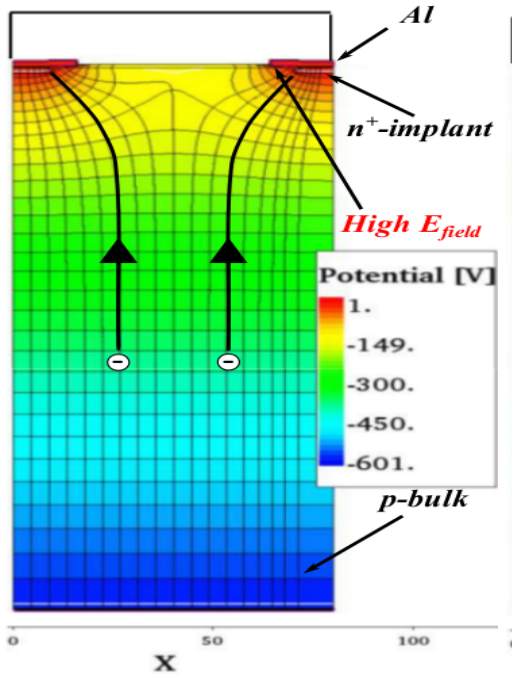

(a)

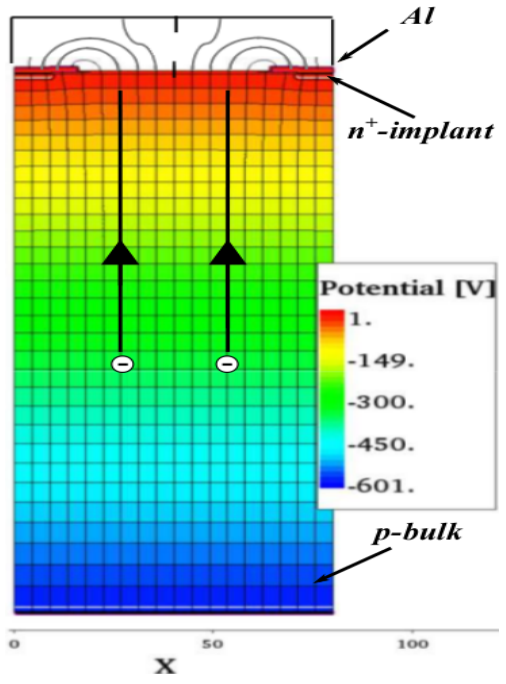

(b)

Figure 1.7: Simulated potential and electric field distributions for a p-spray strip sensor for oxide charge density of (a) $10^{10} \mathrm{~cm}^{-2}$ and (b) $5 \cdot 10^{11} \mathrm{~cm}^{-2}$. Figures taken from $[14,25]$.

the electric field stream lines and the electric potential for a sensor of thickness of $200 \mu \mathrm{m}$, bulk p doping of $3.7 \cdot 10^{12} \mathrm{~cm}^{-3}$ and a p-spray dopant concentration of $2 \cdot 10^{11} \mathrm{~cm}^{-2}$, at a bias voltage of $600 \mathrm{~V}$ for two 
values of oxide charge density at the $\mathrm{Si}-\mathrm{SiO}_{2}$ interface. For the simulation with $N_{o x}=10^{10} \mathrm{~cm}^{-2}$, Fig. 1.7 (a), and in particular air boundary condition, most field lines originate at the readout strips. So for particle at normal incidence, all generated electrons will reach a single readout strip, except from a small effect due to charge diffusion [25]. For the simulation with $N_{o x}=5 \cdot 10^{11} \mathrm{~cm}^{-2}$, Fig. 1.7 (b), also with air boundary condition, the field distribution is very different, as the positive oxide charge density, $N_{o x}$, is bigger than the negative charge density of the p-spray doping, which results in an approximately constant potential at the $\mathrm{Si}-\mathrm{SiO}_{2}$ interface. The electric field component parallel to the interface is small, and electrons, which reach the $\mathrm{Si}_{-} \mathrm{SiO}_{2}$ interface within the typical charge collection time of a few nanoseconds, will drift to the readout strips on much longer time scales.

\subsubsection{Surface parameters and TCAD simulations}

Parameters for TCAD simulations The parameters, relevant for surface effects, and the electrical boundary conditions for simulations, are presented in Fig. 1.8 which shows the cross section of a $\mathrm{p}^{+} \mathrm{n}$ strip sensor [16].

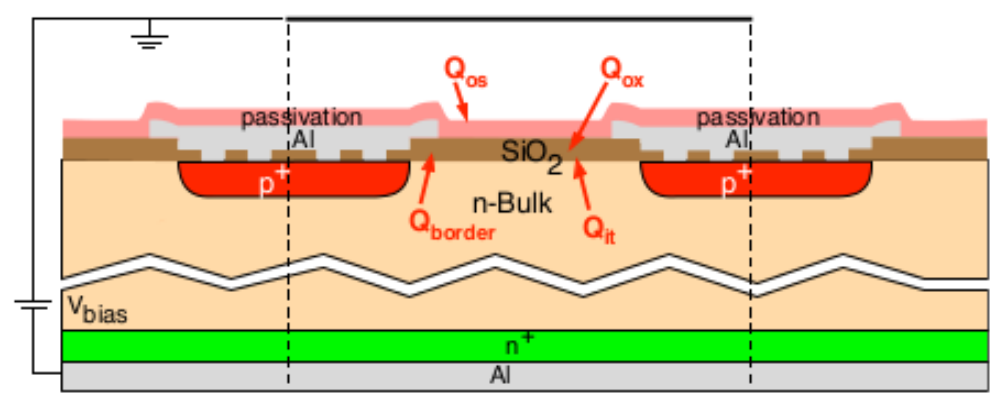

Figure 1.8: Cross section of a strip sensor explaining the parameters relevant for surface effects. Figure taken from [16].

1. The outer-surface charge density, $Q_{o s}$, which can exhibit a time dependence, if the outer-surface resistivity is high.

2. The oxide charge density, $Q_{o x}$, which depends on technology, crystal orientation, dose of ionizing radiation, and the electric field during irradiation.

3 . The border trap charge density, $Q_{b o r d e r}$, which depends on technology and ionizing dose. Border traps are located in the $\mathrm{SiO}_{2}$ within a few $\mathrm{nm}$ of the $\mathrm{Si}_{-} \mathrm{SiO}_{2}$ interface and exchange charges with the $\mathrm{Si}$ with time constants, which can be seconds and even longer.

4. The charged $\mathrm{Si}_{-} \mathrm{SiO}_{2}$ interface trap density, $Q_{i t}$, which depends on technology, ionizing dose and the Fermi energy at the interface.

5. The electrical boundary conditions: Bias voltage at the back side, ground potential on a plane at 0.2 $\mathrm{mm}$ distance parallel to the sensor surface (solid line), and Neumann conditions (air) at the boundaries connecting the centers of the strips with the back plane (dashed lines).

The charge densities depend on position, if the electric field during irradiation or operation is not uniform. So far in the simulations uniform charge densities have been assumed. In previous studies on high-ohmic detector-grade Si $[2,8,26] \mathrm{C}-\mathrm{V}$ measurements on MOS capacitors, without bias voltage during irradiation, from four vendors have been used to determine the effective oxide charge density $N_{o x}^{\text {eff }}=\left(Q_{o x}+Q_{b o r d e r}+\right.$ 
$\left.Q_{i t}\right) / q_{0}$ for ionization doses up to $1 \mathrm{GGy}$. It has been found that before irradiation $N_{o x} \approx 10^{10} \mathrm{~cm}^{-2}$ for $\mathrm{Si}$ with $<100>$ crystal orientation, and $\approx 10^{11} \mathrm{~cm}^{-2}$ for $<111>$. For doses above $10-100 \mathrm{kGy}$, depending on the vendor, $N_{o x}$ saturates between 2 and $8 \cdot 10^{12} \mathrm{~cm}^{-2}$. In addition, the dose dependence of the surface generation current density, $J_{\text {surf }}$, which is related to $Q_{i t}$, has been measured. Before irradiation $J_{\text {surf }}$ is a few $\mathrm{nA} / \mathrm{cm}^{2}$, increases to $\approx 9 \mu \mathrm{A} / \mathrm{cm}^{2}$ at a dose of about $10 \mathrm{kGy}$, and then decreases.

\subsubsection{Impact of surface effects on sensors}

Oxide charge and breakdown voltage The AGIPD sensor $[4,7]$ is an example for the successful optimization of a $500 \mu \mathrm{m}$ thick $\mathrm{p}^{+} \mathrm{n}$ pixel sensor for X-ray doses up to 1 GGy at high operating voltages [16]. Fig. 1.9 (a) shows the voltage dependence of the current measured in the current-collection ring, which surrounds the pixels, for X-ray doses between 0 and $100 \mathrm{MGy}$ for a sensor produced by SINTEF with standard technology. The breakdown voltage decreases from about $900 \mathrm{~V}$ to about $220 \mathrm{~V}$. TCAD simulations explain the cause. For high oxide charges and the standard $\mathrm{SiO}_{2}$ thickness of $700 \mathrm{~nm}$, there is a single narrow highfield peak at the corner of the implantation. Reducing the oxide thickness to $250 \mathrm{~nm}$ results in two field peaks. One at the corner of the implantation and one below the edge of the metal overlap. As a result, the predicted breakdown voltage for $N_{o x}=3 \cdot 10^{12} \mathrm{~cm}^{-2}$ exceeds $1000 \mathrm{~V}$. The sensor has been produced with the optimized $\mathrm{SiO}_{2}$ thickness of $250 \mathrm{~nm}$, and Fig.1.9 (b) shows that the breakdown voltage exceeds $900 \mathrm{~V}$ [7]. The increase in current for the non-irradiated sensor at $800 \mathrm{~V}$ is due to the depletion region reaching the cut edge. It disappears once the sensor has been exposed to a dose of a few $100 \mathrm{~Gy}$, and thus does not present a problem. The observed current is dominated by the surface generation current and is correctly predicted using the surface generation current density measured using Gate Controlled Diodes [8].

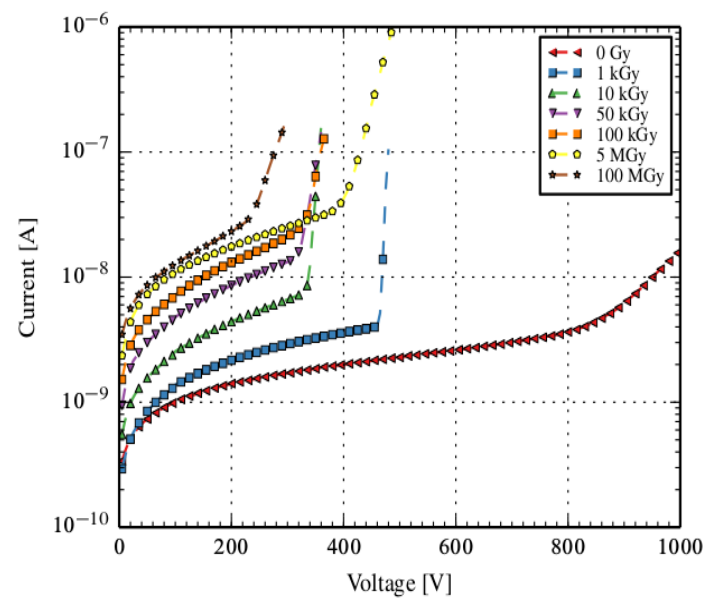

(a)

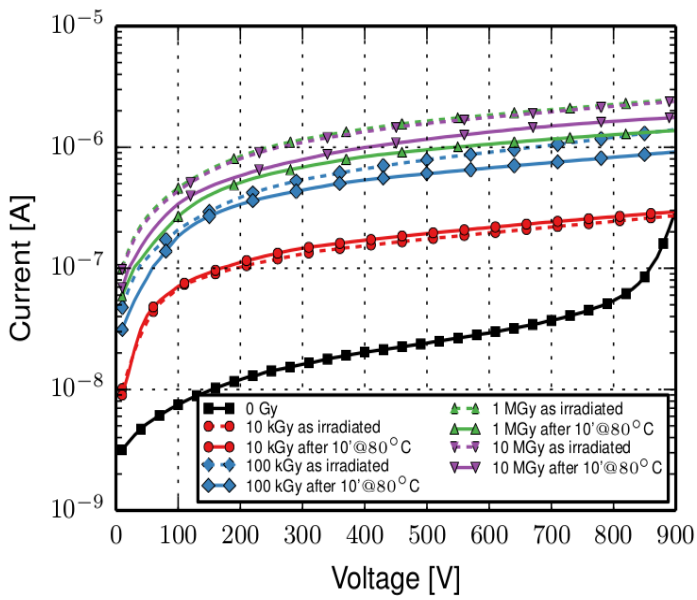

(b)

Figure 1.9: Current voltage characteristics for a pixel sensor for different X-ray doses (a) Standard, and (b) Optimized SINTEF technology. Figures taken from $[4,16]$.

Outer-surface and charge collection In Ref. [23,24] it is reported that, after changing the bias voltage, the charge collection of Si strip sensors changes with time constants, which depend on the relative humidity, $R H$. Fig. 1.10 shows an example. The voltage on a $\mathrm{p}^{+} \mathrm{n}$ Si strip sensor was changed from $500 \mathrm{~V}$ to $200 \mathrm{~V}$, 
and the charge collection for $e h$ pairs, produced by laser light of $670 \mathrm{~nm}$ wavelength injected in-between the read-out strips, has been measured at $20{ }^{\circ} \mathrm{C}$ and $R H \approx 78 \%$, and $<1 \%$.

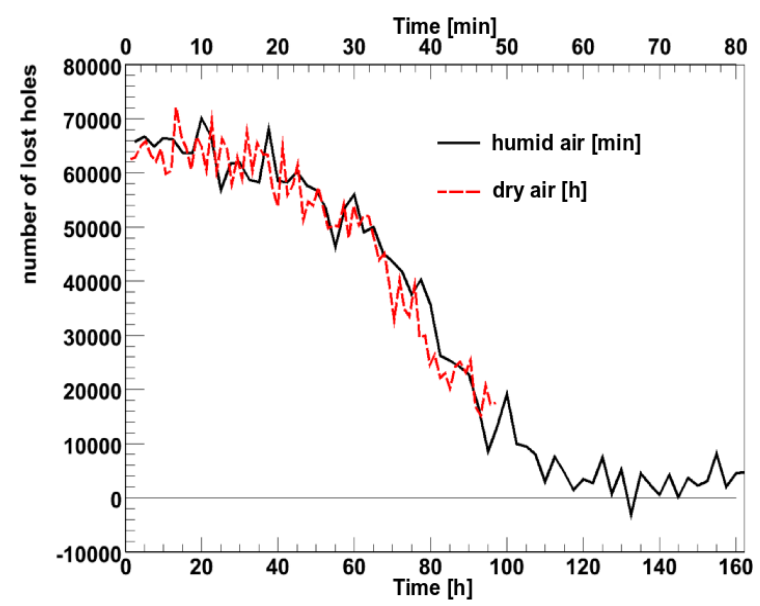

Figure 1.10: Number of "lost" holes versus time for a $\mathrm{p}^{+} \mathrm{n}$ Si strip sensor illuminated with $670 \mathrm{~nm}$ light, after changing the bias voltage from $500 \mathrm{~V}$ to $200 \mathrm{~V}$ for relative humidities of $78 \%$ and $<1 \%$. Figure taken from $[16,24]$.

At $670 \mathrm{~nm}$ the light-attenuation length in $\mathrm{Si}$ is about $3 \mu \mathrm{m}$, and the charge collection is sensitive to the electric field close to the $\mathrm{Si}_{-} \mathrm{SiO}_{2}$ interface. In Fig. 1.10, the time dependence of the number of holes "lost", i.e not recorded as signal in the readout strips within $50 \mathrm{~ns}$, is shown. The time scale on top in minutes is for $R H=78 \%$, and the one at the bottom in hours for $R H<1 \%$. The shape of the curves are similar, however the time constants differ by a factor $\approx 120$. In order to understand this effect the outer-surface resistivity has been determined using the circular Gate Controlled Diode described in Refs. [8, 16], irradiated by X-rays to a dose of 1 GGy. Fig. 1.11 (a) shows $I_{G C D}\left(V_{\text {gate }}\right)$, the current-voltage characteristics, when changing the gate voltage from inversion via depletion to accumulation.

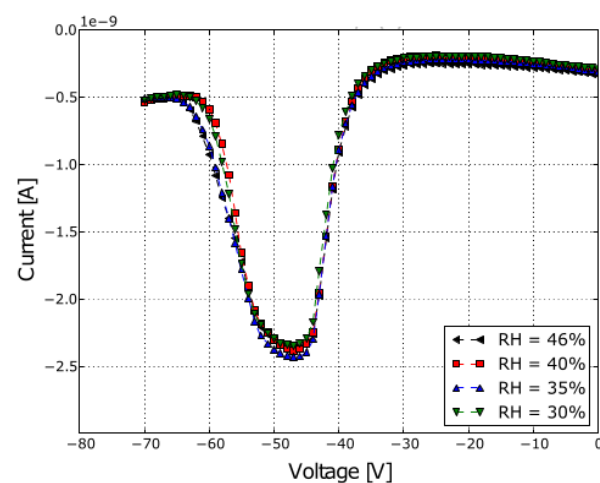

(a)

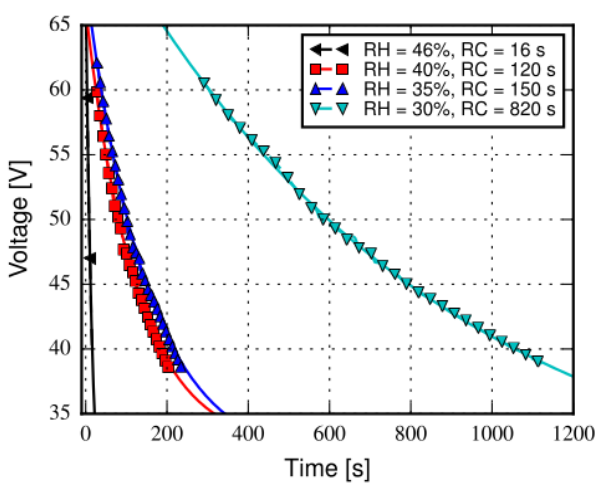

(b)

Figure 1.11: Measurement of the outer-surface resistivity as a function of relative humidity using a GCD, (a) $I_{G C D}$ as a function of $V_{\text {gate }}$ and (b) $V_{\text {gate }}$ as a function of time after the gate was disconnected from the voltage source. Figures taken from [16].

The current-peak is due to the surface-generation current, $I_{\text {surf }}$, from the radiation damaged $\mathrm{Si}_{-} \mathrm{SiO}_{2}$ interface. Then the GCD was biased in inversion, the gate contact disconnected and the time dependence $I_{G C D}(t)$ measured for $R H$ values between $30 \%$ and $46 \%$. Using $I_{G C D}\left(V_{\text {gate }}\right), V_{\text {gate }}(t)$ is derived and shown in 
Fig. 1.11 (b). An exponential dependence is found with time constants varying between $16 \mathrm{~s}$ at $R H=46 \%$ and $820 \mathrm{~s}$ at $R H=30 \%$. The corresponding surface resistivities, $R_{\square}$, are $1.3 \cdot 10^{15} \Omega$ and $65 \cdot 10^{15} \Omega$. At lower $R H$ values, the $R_{\square}$ values are too high, to be determined with this method. To investigate the impact of the outer-resistivity on the charge collection of Si strip sensors, in the TCAD simulation a high-resistivity layer was put on top of the $\mathrm{SiO}_{2}$ layer, the bias voltage ramped from 0 to $-600 \mathrm{~V}$, and the time dependence of the potential simulated. Fig. 1.12 shows the potential and the field lines $60 \mathrm{~s}$ and $120 \mathrm{~min}$ after reaching $-600 \mathrm{~V}$. For the simulation a p-spray isolation of $5 \cdot 10^{11} \mathrm{~cm}^{-2}$, and an effective oxide charge density of $10^{10} \mathrm{~cm}^{-2}$ has been assumed.

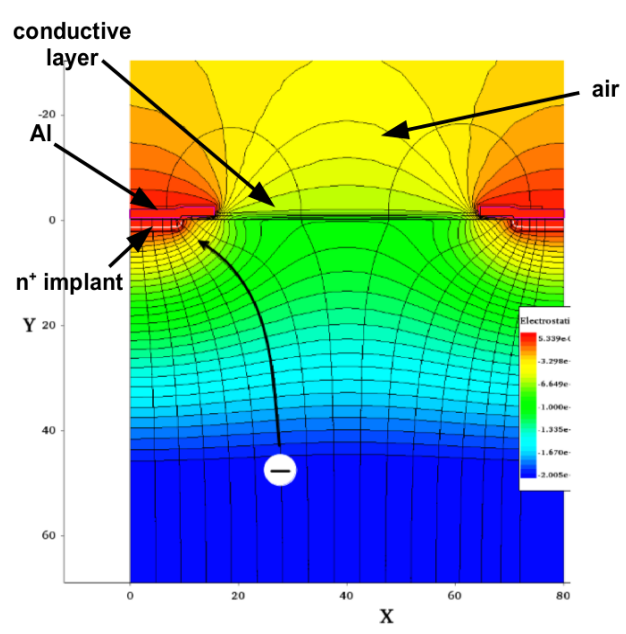

(a)

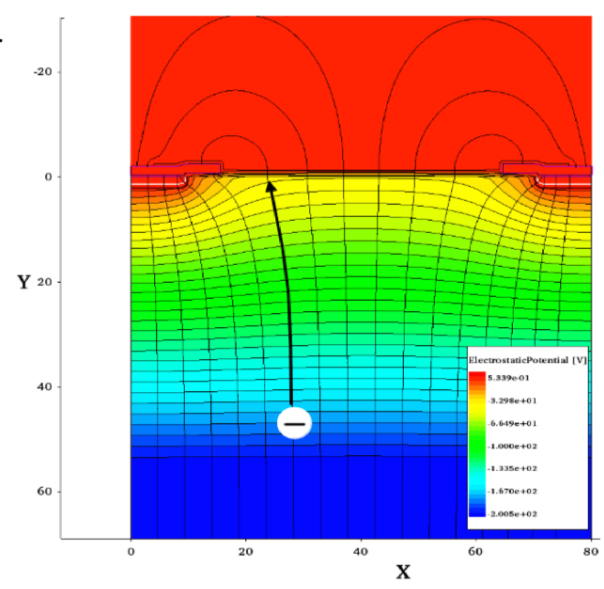

(b)

Figure 1.12: Simulated potential and field-lines in an $n^{+} \mathrm{p}$ silicon strip sensor with outer-surface resistivity $R_{\square}=7 \cdot 10^{14} \Omega$ for (a) 60 seconds, and (b) 120 min after reaching the bias voltage of $-600 \mathrm{~V}$. Figures taken from $[16,27]$.

Whereas after $60 \mathrm{~s}$ practically all field lines in the Si originate at the strip implantations, after 120 min most field lines cross the $\mathrm{Si}_{-} \mathrm{SiO}_{2}$ interface. Thus, immediately after biasing, there are no charges on the outer surface and the electric field has a component along the outer surface, which causes a rearrangement of the surface charges until an equilibrium is reached. The corresponding time constant is proportional to $R_{\square}$, which has a strong humidity dependence.

\subsection{Motivation and structure of this thesis}

So far in the simulations, the effects of surface radiation damage have been parametrized by a uniform and time independent effective oxide charge density, $Q_{o x}^{e f f}=q_{0} N_{o x}^{e f f}$, and a surface current generation density $J_{\text {surf }}$. This is only a first approximation, as the oxide charge density by ionizing radiation depends on the electric field, and the charge state of the border and interface traps on the field and on the Fermi level at the $\mathrm{Si}-\mathrm{SiO}_{2}$ interface. In addition, possible short time effects during irradiation have not been considered. In the present thesis mainly are presented:

1. Effects during and shortly after X-ray irradiation. 
2. The dependence of $N_{o x}^{e f f}$ on the direction of the field at the $\mathrm{Si}_{-} \mathrm{SiO}_{2}$ interface using $\mathrm{p}$ - and n-MOSFETs.

3. The charging and discharging of border traps when the field direction is reversed.

4. An attempt to separate $Q_{o x}$ and $Q_{i t}$ and to determine $D_{i t}$, the energy distribution of the interface traps in the Si bandgap using the subthreshold current technique based on the Brews charge sheet model of the long channel MOSFET.

5. The annealing dependence of the above quantities.

The results presented in this thesis can be used to improve the simulation of segmented silicon sensors by taking into account the field dependence of the surface radiation damage. In addition information is produced to estimate short- and long-term effects after changing the biasing of segmented silicon sensors. 


\section{Physics of basic semiconductor and device theory}

\subsection{Metal Oxide Semiconductor MOS structures}

This chapter summarizes the physics of the MOS structures, MOS Capacitor (MOSC) and MOS Field Effect Transistor (MOSFET). The MOSC is a good introduction to the MOSFET because it allows the action of the gate electrode to be analyzed without the complications of current flow $[28,29]$.

\subsubsection{MOS Capacitor}

The MOSC consists of a silicon substrate with a thermally oxidized silicon dioxide surface. On the top of the silicon dioxide is the conducting Al metal as shown in Fig. 2.1.

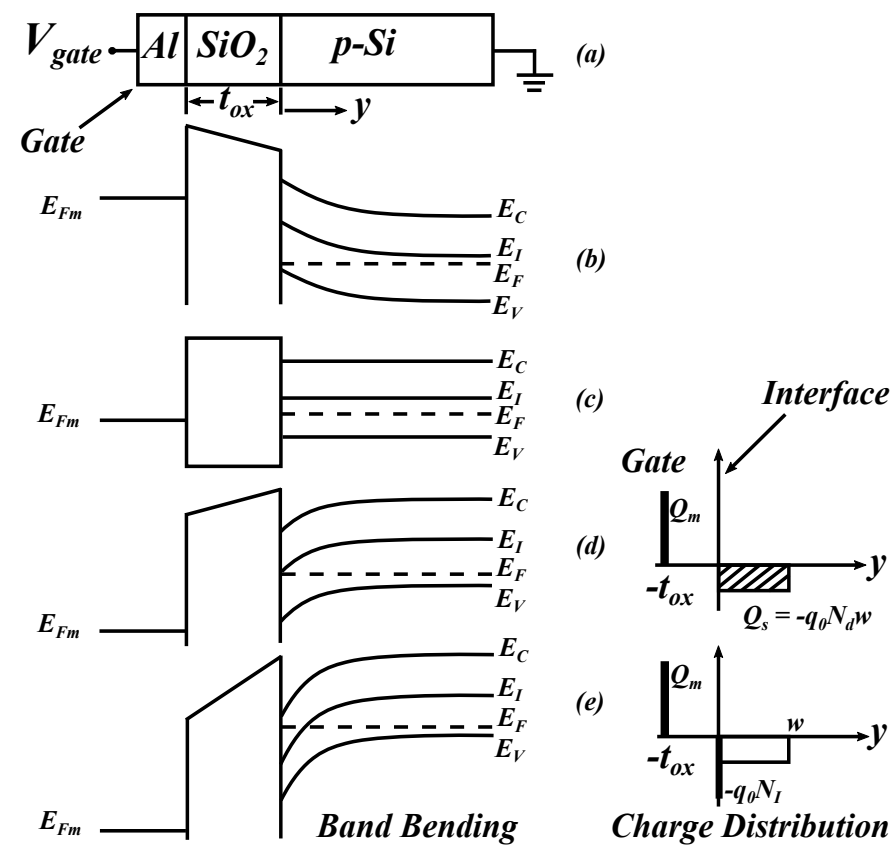

Figure 2.1: Band bending and charge distribution in the MOS Capacitor for various biases: (a) structure, (b) accumulation, (c) flatbands, (d) depletion, (e) inversion. $E_{C}=$ conduction band, $E_{V}=$ valence band, $E_{I}=$ intrinsic level, $E_{F}=$ Fermi level of silicon, and $E_{F m}=$ Fermi level of gate. Figure adapted from [28].

The MOSC is always in thermal equilibrium, even with dc bias applied [30]. The bias regimes and the simplified solution of Poisson's equation to determine band bending and carrier densities are presented. 
Considering a p-type substrate $\mathrm{Si}$ the majority carriers are holes and the Fermi level is in the lower half of the bandgap. Four different situations exist depending upon the bias applied to the gate.

Accumulation If a negative bias is applied to the gate, electron energies are raised in the metal relative to the substrate. The Fermi level $E_{F m}$ of the metal lies above its equilibrium position. Also negative $V_{\text {gate }}$ results in a negative charge on the gate, so a positive charge is induced at the silicon surface. For p-type silicon (p-Si) holes are accumulated at the surface. As the hole concentration increases at the surface, the difference between the intrinsic level $E_{I}$ and the Fermi level $E_{F}$ increases resulting in the bands bending upwards as shown in Fig. 2.1 (b).

Flatbands At flatbands the silicon bands are flat, right up to the $\mathrm{Si}_{-} \mathrm{SiO}_{2}$ interface, Fig. 2.1 (c). The silicon is neutral everywhere, because the holes $(+)$ exactly balance the acceptor dopant ions $(-)$. The gate bias for which flatbands occurs is called the flatband voltage, $V_{f b}$.

Depletion If a positive bias is applied to the gate, then the positive holes are repelled from the silicon surface. The energy bands bend down, representing the energy levels as (e.g for the conduction band)

$$
E_{c}(y) / q_{0}=E_{c}(\infty) / q_{0}-\phi(y)
$$

where $E_{c}(y)$ is the conduction band edge and $\phi(y)$ is the band bending at position $y$ from the interface. Because the holes are depleted near the interface, there is a region containing primarily acceptor dopant ions, $N_{d}$ per unit volume, as shown in Fig. 2.1 (d). The band bending potential, $\phi(y)$ then satisfies Poisson's equation in the depletion approximation:

$$
\begin{aligned}
\frac{d^{2} \phi}{d y^{2}} & =\frac{q_{0} N_{d}(y)}{\varepsilon_{S i} \varepsilon_{0}}, & 0<y<w \\
& =0, & w<y
\end{aligned}
$$

Where $\varepsilon_{S i}=11.9$ is the Si dielectric constant and $\varepsilon_{0}=8.85 \cdot 10^{-14} \mathrm{~F} / \mathrm{cm}$ is the permittivity of empty space. If the dopant ions are uniformly distributed so $N_{d}$ is independent of y, then Eq. 2.2 can be integrated to yield

$$
\phi(y)=\phi_{s}(1-y / w)^{2}
$$

where $\phi_{s}$ is the value of $\phi(y)$ at $y=0$ and $\mathrm{w}$ is the depletion width, the depth to which the hole density is negligible. The first and second derivatives of Eq. 2.3 are written

$$
\frac{d \phi}{d y}=2 \phi_{s}(1-y / w)(-1 / w)
$$




$$
\begin{aligned}
\frac{d^{2} \phi}{d y^{2}} & =\frac{2 \phi_{s}}{w^{2}} \\
& =\frac{q_{0} N_{d}}{\varepsilon_{S i} \varepsilon_{0}}
\end{aligned}
$$

To simplify, $\beta=q_{0} / k T$ is the reciprocal of the thermal voltage, $k T / q_{0}(\approx 0.025 \mathrm{~V}$ at $290 \mathrm{~K})$. So $w$ is calculated from

$$
w=\sqrt{2} L_{D}\left(\beta \phi_{s}\right)^{1 / 2}
$$

where $L_{D}$ is the bulk Debye length

$$
L_{D}=\sqrt{\frac{\varepsilon_{S i} \varepsilon_{0}}{\beta q_{0} N_{d}}} .
$$

In particular Eq. 2.4 shows that the field at the depletion edge $(y=w)$ vanishes. It is expected because there is no charge in the region $y>w$. This bulk region is in the flatband configuration where the hole density equals the acceptor density.

Inversion If a large enough positive bias is applied to the gate, then the conduction band edge, $E_{c}(0)$, approaches the Fermi level, $E_{F}$, in the vicinity of the $\mathrm{Si}_{-} \mathrm{SiO}_{2}$ interface. When this happens electrons appear in the immediate vicinity of the interface, in an inversion layer, as shown in Fig. 2.1 (e). Once the inversion layer forms, additional positive bias on the gate simply draws more electrons into the inversion layer. The negatively charged electrons balance the additional positive gate charge, and the depletion width, $w$, no longer increases because the band bending $\phi_{s}$ in Eq. 2.6 becomes pinned or clamped. Consequently, $w$ also becomes pinned at the value it held just before the inversion layer formed. To treat the inversion layer electrons they have to be included in the Poisson Eq. 2.2. The hole density is

$$
p=n_{i} e^{\left(-\beta \phi+\beta \phi_{b}\right)}
$$

where $n_{i}=$ the carrier density in intrinsic material $\left(\approx 10^{10}\right.$ carriers per $\mathrm{cm}^{2}$ at $\left.290 \mathrm{~K}\right)$ and $\phi_{b}$ is the Fermi level in electron volts $(\mathrm{eV}): \phi_{b}=E_{F} / q_{0}$. The law of mass action implies that at thermal equilibrium

$$
p n=n_{i}^{2}
$$

Using Eqs. 2.8 and 2.9 the electron density is

$$
n=n_{i} e^{\left(\beta \phi-\beta \phi_{b}\right)} .
$$

At flatbands, in the neutral silicon $p=N_{d}$, using Eq. 2.9 the $\phi_{b}$ is calculated from 


$$
\beta \phi_{b}=\ln \left(N_{d} / n_{i}\right)
$$

The electron density in Eq. 2.10 gives an additional term in the Poisson Eq. 2.2,

$$
\begin{aligned}
\frac{d^{2} \phi}{d y^{2}} & =\frac{q_{0} N_{d}(y)}{\varepsilon_{S i} \varepsilon_{0}}+\frac{q_{0} n_{i}}{\varepsilon_{S i} \varepsilon_{0}} e^{\left(\beta \phi-\beta \phi_{b}\right)}, & 0<y<w \\
& =0, & y>w
\end{aligned}
$$

For uniform doping, multiply by $(d \phi / d y)$ and use the identity

$$
\frac{1}{2} \frac{d}{d y}\left(\frac{d \phi}{d y}\right)^{2}=\left(\frac{d \phi}{d y}\right)\left(\frac{d^{2} \phi}{d y^{2}}\right)
$$

Then Eq. 2.12 becomes

$$
\frac{1}{2} \frac{d}{d y}\left(\frac{d \phi}{d y}\right)^{2}=\frac{1}{\left(\beta L_{D}\right)^{2}} \frac{d}{d y}\left[\beta \phi+\left(n_{i} / N_{d}\right)^{2} e^{(\beta \phi)}\right]
$$

Integrating from the interface, $y=0$, to the depletion edge, $y=w$

$$
\left(\frac{d \phi}{d x}\right)_{w}^{2}-\left(\frac{d \phi}{d x}\right)_{0}^{2}=\frac{1}{\left(\beta L_{D}^{2}\right)}\left[\beta \phi+\left(n_{i} / N_{d}\right)^{2} e^{(\beta \phi)}\right]_{0}^{w}
$$

Now at $y=w$ both $\phi$ and $(d \phi / d y)$ vanish. At $y=0, \phi=\phi_{s}$. So far, Eq. 2.15 becomes

$$
-(d \phi / d y)_{0}=\sqrt{2}\left(\beta L_{D}\right)^{-1}\left\{\beta \phi_{s}+\left(n_{i} / N_{d}\right)^{2}\left[e^{\left(\beta \phi_{s}\right)}-1\right]\right\}^{1 / 2}
$$

The inversion layer carrier density per unit area, $N_{I}$, can be calculated by integrating Eq. 2.10. Then the potential is needed as a function of distance. To proceed from Eq. 2.16 to find the potential requires a numerical integration. According to Ref. [28] numerical integration shows that the minority carriers in the inversion layer are very close to the interface, probably within $30-300 \AA$, depending on gate bias. Using an approximation that the inversion layer is a charge sheet of infinitesimal thickness, the $N_{I}$ can be calculated easily using Eq. 2.16. The total silicon charge per unit area is $Q_{s}$, the charge in the depletion is $\left(-q_{0} N_{d} w\right)$ [the charge per unit volume, $\left(-q_{0} N_{d}\right)$, times $w$ the depletion width]. Then the difference, total charge less dopant ion charge, is the charge due to inversion layer electron density per unit area, $-q_{0} N_{I}$ :

$$
-q_{0} N_{I}=Q_{s}-\left(-q_{0} N_{d} w\right)
$$

The width $w$ is calculated from Eq. 2.6 and $Q_{s}$ is found from Eq. 2.16 using Gauss's law, which states that the field times the dielectric permittivity is equal to the charge giving rise to this field 


$$
Q_{s}=-\left(\varepsilon_{S i} \varepsilon_{0} / L_{D}\right)\left(k T / q_{0}\right) \sqrt{2}\left\{\beta \phi_{s}+\left(n_{i} / N_{d}\right)^{2}\left[e^{\left(\beta \phi_{s}\right)}-1\right]\right\}^{1 / 2}
$$

For convenience the (-1) after $e^{\left(\beta \phi_{s}\right)}$ can be omitted because the exponential is so large in strong inversion that $(-1)$ makes no difference, and in weak inversion $\left(n_{i} / N_{d}^{2}\right)$ is so small that the entire minority carrier term can be dropped. So far using the charge sheet approximation the $N_{I}$ is calculated from

$$
N_{I} \cong N_{d} \sqrt{2} L_{D}\left\{\left[\beta \phi_{s}+\left(n_{i} / N_{d}\right)^{2} e^{\left(\beta \phi_{s}\right)}\right]^{1 / 2}-\left[\beta \phi_{s}\right]^{1 / 2}\right\}
$$

Using Eq. 2.19 the inversion layer carrier density per unit area $N_{I}$, as a function of band bending is shown in Fig. 2.2.

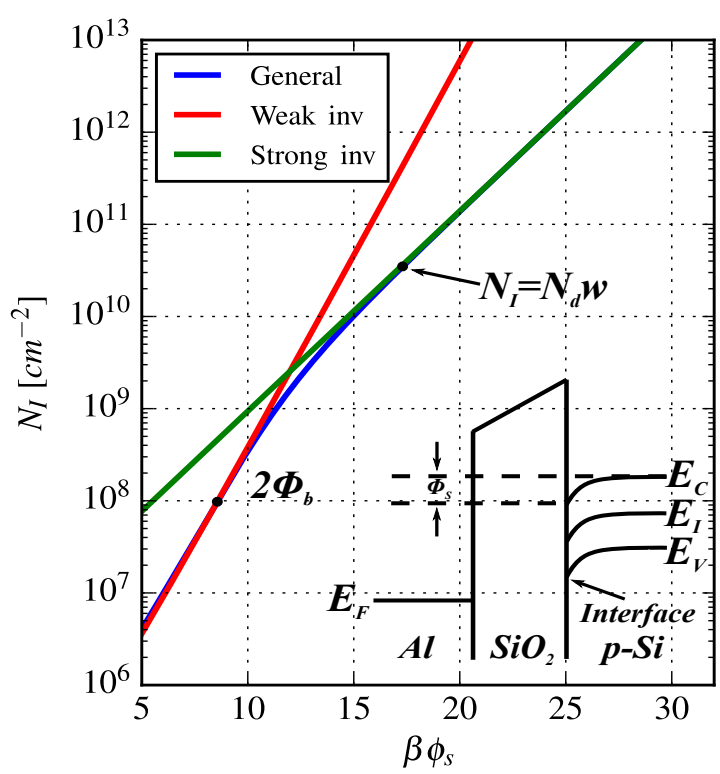

Figure 2.2: Inversion layer carrier density per unit area $N_{I}$ as a function of band bending $\phi_{s}$. The red line is for weak inversion, the green line is for strong inversion, and the blue line is the general case.

The figure presents two regimes, for $N_{d}=4 \cdot 10^{11} \mathrm{~cm}^{-3}$ :

i) Weak inversion. In the weak inversion regime, $N_{I}$ is small compared to the depletion layer charge per unit area. The square root in Eq. 2.19 is expanded treating $\left(n_{i} / N_{d}\right)^{2} e^{\left(\beta \phi_{s}\right)}$ small compared to $\beta \phi_{s}$. Using the expansion

$$
(1+x)^{1 / 2} \approx 1+(1 / 2) x+\ldots
$$

the $N_{I}$ in weak inversion is written

$$
N_{I} \approx N_{d} L_{D}\left(n_{i} / N_{d}\right)^{2} e^{\left(\beta \phi_{s}\right)} /\left(2 \beta \phi_{s}\right)^{1 / 2}
$$

ii) Strong inversion. In strong inversion, $N_{I}$ is very large compared to the depletion layer charge per unit area. The exponential in Eq. 2.19 is large compared to $\beta \phi_{s}$ and $N_{I}$ in strong inversion becomes 


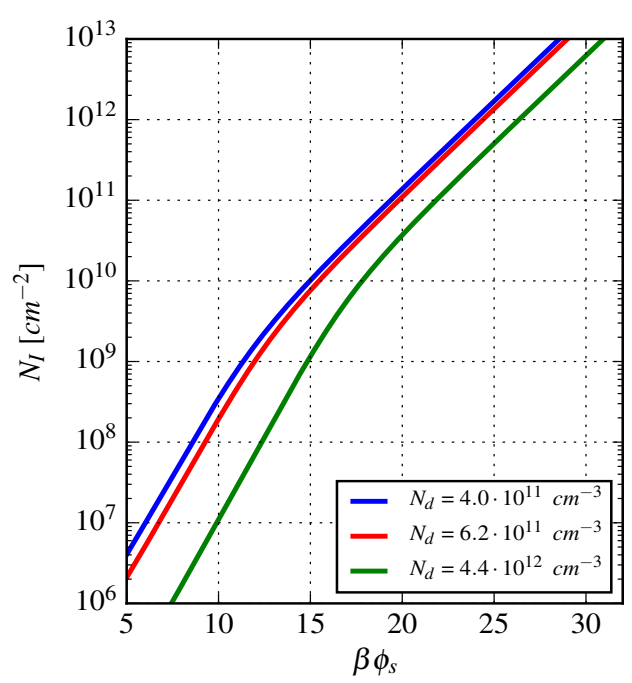

Figure 2.3: Inversion layer carrier density per unit area $N_{I}$ as a function of band bending $\phi_{s}$ for three different doping concentration values $N_{d}$.

$$
N_{I} \approx \sqrt{2} L_{D} n_{i} e^{\left(\beta \phi_{s} / 2\right)}
$$

The inversion layer carrier density per unit area $N_{I}$ as a function of band bending $\phi_{s}$, for typical doping concentrations $N_{d}$, commonly used for silicon sensors, is presented in Fig. 2.3. The increase of $N_{d}$, shifts the strong inversion condition to higher $\phi_{s}$ values.

Gate bias dependence of band bending In inversion the band bending $\phi_{s}$ becomes pinned, independent of gate bias. According to electrostatics, the electric field times the dielectric permittivity is continuous at the $\mathrm{Si}-\mathrm{SiO}_{2}$ interface. The electric field at the silicon side of the interface is given by Eq. 2.16 and on the oxide side of the interface is given by Eq. 2.23

$$
E_{o x}=\left(V_{\text {gate }}-\phi_{s}\right) / t_{o x}
$$

where $V_{\text {gate }}$ is the gate bias, $\phi_{s}$ the band bending and $t_{o x}$ the oxide thickness. Continuity of displacement across the interface (i.e., of the product of field times dielectric permittivity), using Eqs. 2.16 and 2.23, provides

$$
C_{o x}\left(V_{\text {gate }}-\phi_{s}\right)=\sqrt{2}\left(k T / q_{0}\right) C_{f b, S i}\left(\beta \phi_{s}+\left(n_{i} / N_{d}\right)^{2}\left[e^{\left(\beta \phi_{s}\right)}-1\right]\right)^{1 / 2}
$$

where the definition for the oxide capacitance per unit area, $C_{o x}$, is

$$
C_{o x}=\varepsilon_{o x} \varepsilon_{0} / t_{o x}
$$

and for $C_{f b, S i}$ 


$$
C_{f b, S i}=\varepsilon_{S i} \varepsilon_{0} / L_{D}
$$

is the capacitance per unit area of the silicon portion of the MOS capacitor at flatbands. The $C_{f b, S i}$ is related to screening at the silicon surface by majority carriers, for example in response to a small charge uniformly distributed on the gate. From Eq. 2.24 can be calculated the relation between gate bias and band bending. At low gate biases $\phi_{s}$ increases rapidly with gate bias. At larger gate biases $\phi_{s}$ hardly changes with gate bias, $\phi_{s}$ become pinned. The pinning occurs when strong inversion sets in, the point where $N_{I}=N_{d} w$ (inversion layer charge equals depletion layer charge). The change in behavior of $\phi_{s}$ as a function of $V_{\text {gate }}$ is close to this condition, Fig. 2.4. Also shown as a dot the condition

$$
\phi_{s}=2 \phi_{b}
$$

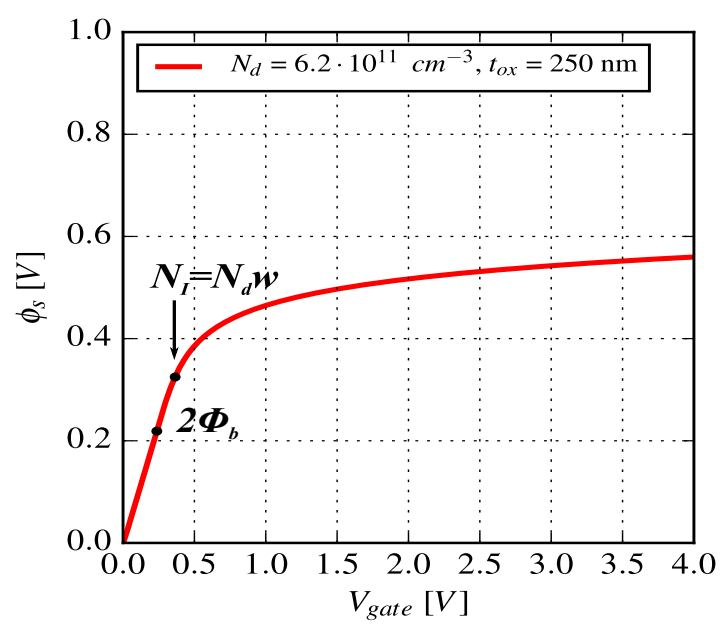

Figure 2.4: Band bending $\phi_{s}$ as a function of gate voltage $V_{\text {gate }}$. The arrow illustrates the condition $N_{I}=N_{d} w$. The dot indicates $\phi_{s}=2 \phi_{b}$.

The $V_{\text {gate }}$ dependence as a function of $\phi_{s}$, for doping concentrations $N_{d}$ and oxide thicknesses $t_{o x}$ commonly used in the design of silicon sensors, is presented in Fig. 2.5. It is clear, that the transition occurs near the Lindner potential $\phi_{L}$ [31] which marks the value of $\phi_{s}$ at which the depletion layer charge and the minority carriers make equal contributions to the field. The condition in Eq. 2.27 is near the condition $N_{I}=N_{d} w$ and referred as threshold condition (the condition which divides strong inversion layers from weak inversion layers). The corresponding gate bias is called threshold voltage, $V_{t h}$, and from Eqs. 2.24 and 2.11 is extracted

$$
V_{t h}=2 \phi_{b}+\sqrt{2}\left(k T / q_{0}\right)\left(C_{f b} / C_{o x}\right)\left(2 \beta \phi_{b}\right)^{1 / 2}
$$

Capacitance of the MOS structure The capacitance of any system is the ratio of the variation in charge to the variation in small signal applied voltage. The capacitance in the silicon bulk below the gate is calculated from $C_{s}=-d Q_{s} / d \phi_{s}$ where $Q_{s}$ is obtained by keeping the majority carrier density in Poisson's equation and following the same procedure to find $Q_{s}$ as presented above. So far 


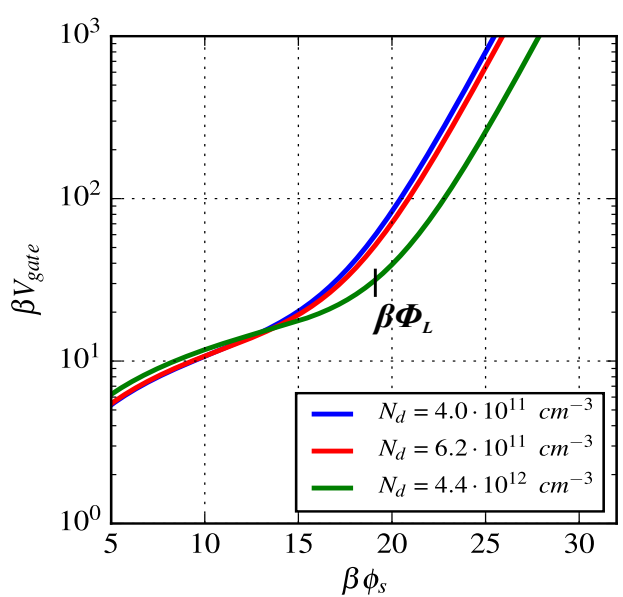

(a)

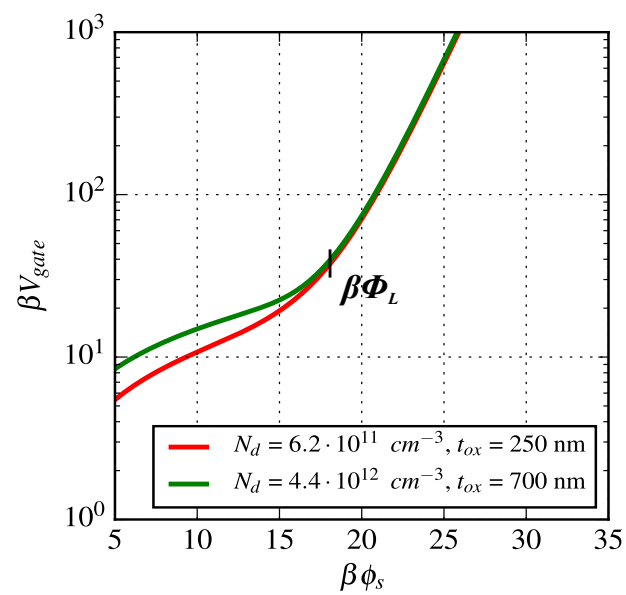

(b)

Figure 2.5: (a) $\beta V_{\text {gate }}$ as a function of $\beta \phi_{s}$ for three different $N_{d}$ values and $t_{o x}=250 \mathrm{~nm}$, and (b) $\beta V_{\text {gate }}$ as a function of $\beta \phi_{s}$ for two different $N_{d}$ and $t_{o x}$ values.

$$
\begin{array}{r}
C_{s}=C_{f b, S i}\left\{1-e^{\left(-\beta \phi_{s}\right)}+\left(n_{i} / N_{d}\right)^{2}\left[e^{\left(\beta \phi_{s}\right)}-1\right]\right\} \\
\cdot\left\{2\left[e^{\left(-\beta \phi_{s}\right)}+\beta \phi_{s}-1+\left(n_{i} / N_{d}\right)^{2}\left(e^{\left(\beta \phi_{s}\right)}-\beta \phi_{s}-1\right)\right]\right\}^{-1 / 2}
\end{array}
$$

where $C_{s}\left(\phi_{s}=0\right)=C_{f b, S i}$. Eq. 2.29 provides the capacitance $C_{s}$, assuming a small signal variation in band bending, $\phi_{s}$. Actually as the gate bias is varied, the relation between the gate bias and the corresponding band bending variation is obtained from

$$
\begin{gathered}
C_{o x}\left(V_{\text {gate }}-\phi_{s}\right)=-Q_{s} \\
C_{o x}\left(1-\frac{d \phi_{s}}{d V_{\text {gate }}}\right)=C_{s} \frac{d \phi_{s}}{d V_{\text {gate }}} \\
\left(\frac{d \phi_{s}}{d V_{\text {gate }}}\right)=C_{o x} /\left[C_{o x}+C_{s}\right]
\end{gathered}
$$

Finally, the capacitance of the entire MOS structure is

$$
\begin{aligned}
C_{M O S} & =-d Q_{s} / d V_{\text {gate }} \\
& =\left(-d Q_{s} / d \phi_{s}\right)\left(d \phi_{s} / d V_{\text {gate }}\right) \\
& =C_{s} C_{o x}\left[C_{s}+C_{o x}\right]^{-1}
\end{aligned}
$$

The calculated capacitance $C_{M O S} / C_{O x}$ as a function of $V_{\text {gate }}$ for three different doping concentration values $N_{d}$ is presented in Fig. 2.6. 


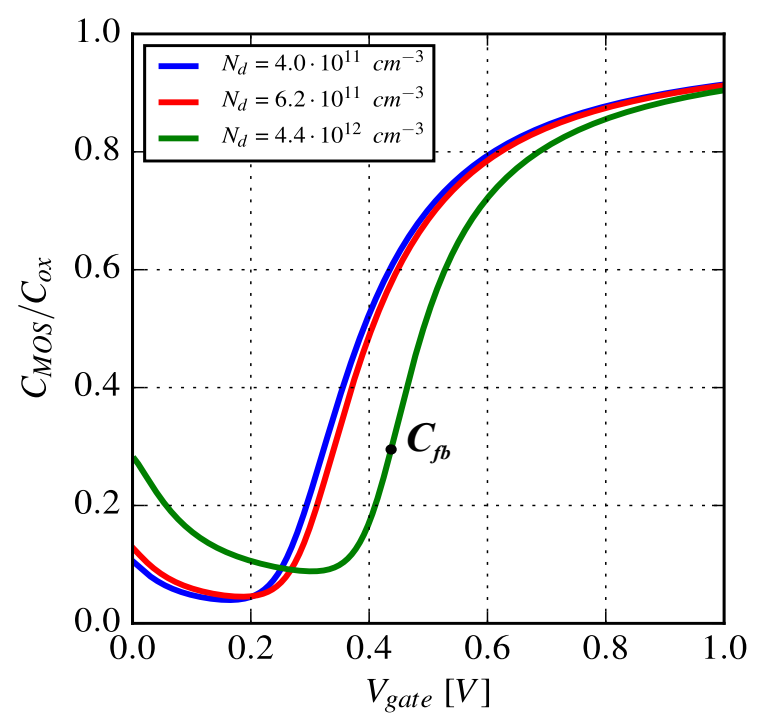

Figure 2.6: $C_{M O S} / C_{o x}$ as a function of $V_{g a t e}$ for three different doping concentration values $N_{d}$ and $t_{o x}=$ $250 \mathrm{~nm}$. The $C_{f b}$ is calculated for $N_{d}=4.4 \cdot 10^{12}$.

The flatband capacitance $C_{f b}$ of the MOS structure is calculated from

$$
C_{f b}=C_{f b, S i} C_{o x}\left[C_{f b, S i}+C_{o x}\right]^{-1} .
$$

In addition the consideration of gate bias as a function of band bending requires a correction. According to Eq. 2.24, when $\phi_{s}=0$

$$
\left(\frac{d \phi_{s}}{d V_{\text {gate }}}\right)=C_{o x} /\left[C_{o x}+C_{s}\right]
$$

the gate bias $V_{\text {gate }}=0$. In practice, this is not found to be the case. Instead, $\phi_{s}=0$ occurs for a gate bias $V_{\text {gate }}=V_{f b}$, called the flatband voltage. So a replacement of $V_{g a t e}$ in Eq. 2.24 by $V_{\text {gate }}-V_{f b}$ the $V_{t h}$ in Eq. 2.28 becomes

$$
V_{t h}=V_{f b}+2 \phi_{b}+\sqrt{2}\left(k T / q_{0}\right)\left(C_{f b, S i} / C_{o x}\right)\left(2 \beta \phi_{b}\right)^{1 / 2}
$$

Theoretical estimates of $V_{f b}$ are very inaccurate, so experimental values usually are adopted. Two important physical factors that cause $V_{f b}$ are the work function differences and the oxide charge, including fixed oxide charge and charged interface traps. If the work of an electron to leave the metal is $q_{0} \phi_{m}$ and to leave the silicon is $q_{0} \phi_{s}$, then the substrate charges up until it reaches a voltage relative to the gate of $\Delta V$,

$$
\begin{gathered}
\Delta V=-\left(\phi_{s}-\phi_{m}\right)=\phi_{m s} \\
\phi_{m s}=\phi_{m}-\left(\chi+\frac{E_{g}}{2 q_{0}}-\phi_{b}\right) \quad \text { for n-type } \\
\phi_{m s}=\phi_{m}-\left(\chi+\frac{E_{g}}{2 q_{0}}+\phi_{b}\right) \quad \text { for p-type }
\end{gathered}
$$


where $\chi$ is the semiconductor electron affinity and $E_{g}$ the Si bandgap. The work function difference between the Al-metal gate and bulk $\mathrm{Si}$ (n- and p-type), $\phi_{m s}$, as a function of the doping concentration $N_{d}$, is presented in Fig. 2.7.

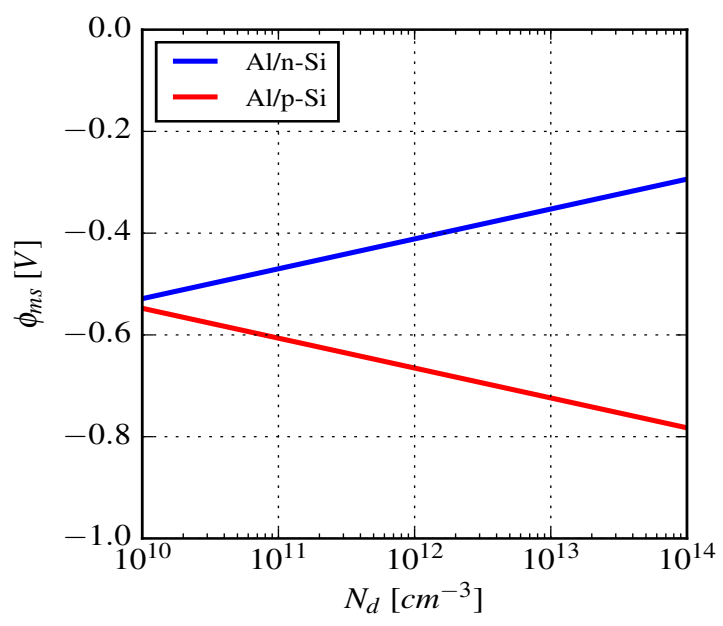

Figure 2.7: $\Phi_{m s}$ as a function of $N_{d}$ for $\mathrm{Al} / \mathrm{n}$ - and $\mathrm{Al} / \mathrm{p}$-type $\mathrm{Si}$.

\subsubsection{MOS Field Effect Transistor MOSFET}

The MOSFET structure is a MOSC with two additions, a source and a drain located on opposite sides of the gate electrode. The source and drain are regions in the silicon doped oppositely to the silicon under the gate. So the source and drain make p-n junctions with the substrate. The source and drain are the contacts of a switch which is turned on and off electrically by a voltage on the gate electrode. For a gate bias which depletes the silicon surface of mobile carriers, the source and drain are isolated. The switch is off. Only a very low current will flow from source to drain when a voltage is applied between them. For gate biases which invert the surface, the switch will be on, and a large current can flow between source and drain. Gate biases which allow current flow are referred as above threshold. For these biases, the voltage step at the p-n junctions decreases near the interface because this region contains carriers of the same type as the source and drain regions. In Fig. 2.8 is shown the band bending in the n-MOSFET. At the drain, the band bending is large and electrons tend to be collected. The passage of electrons from the source to the drain is impeded by a barrier, as shown. This barrier is lowest at the interface, and is controlled by the gate bias. For nMOSFET a more positive gate bias lowers this barrier. The junction between drain and substrate is widened by the reverse bias applied. The Pao-Sah model [32-35] describes the MOSFET structure very well, and the introduction into this model of the charge sheet approximation of the inversion layer, is making the model not only accurate but simple. In addition, the charge sheet model $[28,36]$ is the best basis for understanding the long channel device [37-42].

The accommodation of carrier densities to the current flow is expressed by an adjustment of the Fermi level at each location in the channel. So far, the Fermi level is replaced by a quasi-Fermi level that is not the same everywhere, but varies from point to point to allow the required current to flow. The total current density $j$, is the sum of the diffusion $j_{d i f f}$ and the drift component $j_{d r i f t}$, 


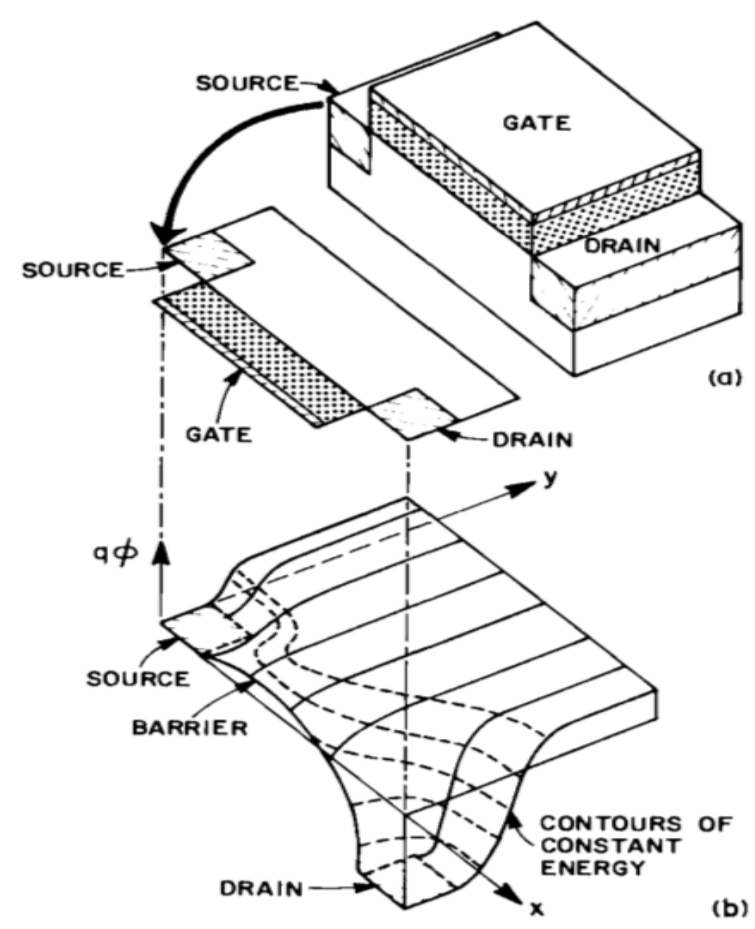

Figure 2.8: (a) MOSFET structure with section cut away and dropped and (b) Band bending plotted below the dropped section, the barrier to electron injection from the source into the channel has been indicated. Figure taken from [28].

$$
j=-q_{0} \mu n \frac{d \phi_{F_{n}}}{d x}
$$

is the basic equation to compute current-voltage characteristics, where $\mu$ is the mobility and $n$ is the electron density for n-channel devices (p-type substrate). The current carried by the entire inversion layer is

$$
I=-W q_{0} \mu N_{I} \frac{d \phi_{F_{n}}}{d x}
$$

where $\phi_{F_{n}}$ varies with $x$, but is independent on the distance from the interface, $y$. The quantity $N_{I}$ in Eq. 2.40, is the inversion carrier density per unit area, already described in Eq. 2.19, however modified to account for the quasi-Fermi level. Backside voltage $V_{b s}$ reduces $N_{I}$, so a larger gate bias is necessary to cause inversion. In strong inversion $\phi_{s} \rightarrow \phi_{s}+V_{b s}$ to maintain $N_{I}$ constant. Using this result in Eq. 2.36, the threshold voltage $V_{t h}$ relative to the source, which is at bias $V_{b s}$ above the substrate

$$
V_{t h}=V_{f b}+2 \phi_{b}+\sqrt{2}\left(k T / q_{0}\right)\left(C_{f b, S i} / C_{o x}\right)\left(2 \beta \phi_{b}+\beta V_{b s}\right)^{1 / 2} .
$$

The threshold voltage $V_{t h}$, is plotted as a function of the parameter $\alpha=\sqrt{2}\left(C_{f b, S i} / C_{o x}\right)$. The range of gate oxide thicknesses and doping levels is contained within $100 \mathrm{~nm} \leq t_{o x} \leq 1000 \mathrm{~nm}$ and $10^{10} \mathrm{~cm}^{-3}$ $\leq N_{d} \leq 10^{14} \mathrm{~cm}^{-3}$ respectively. MOSFET devices within these bounds correspond to the shaded area in Fig. 2.9. $V_{t h}$ is negative for $\alpha$ values in the range up to $\approx 1.8$. They are normally on, even at zero gate bias. 


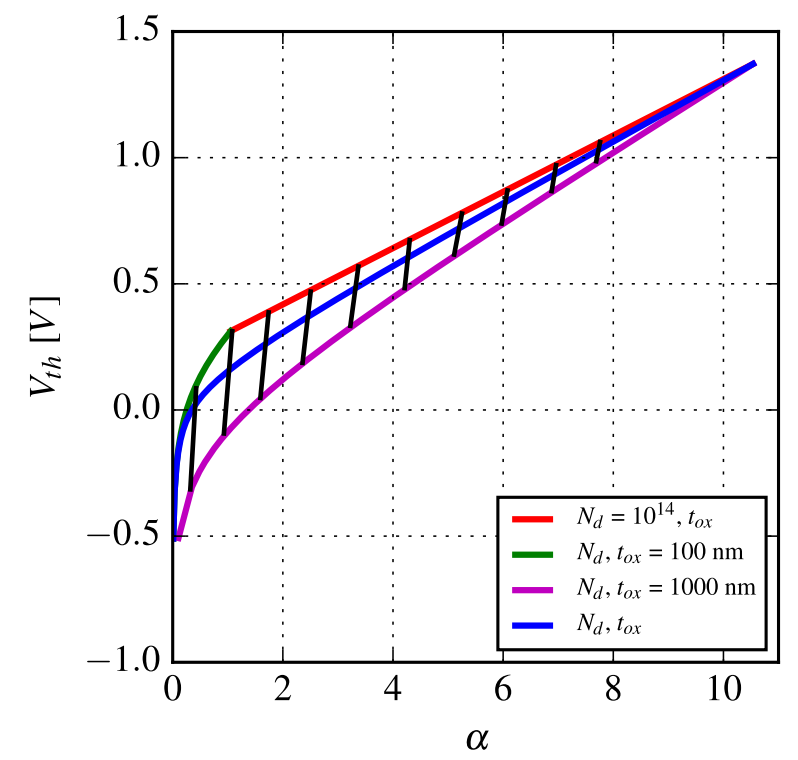

Figure 2.9: Threshold voltage $V_{t h}$ as a function of $\alpha$ for $100 \mathrm{~nm} \leq t_{o x} \leq 1000 \mathrm{~nm}$ and $10^{10} \mathrm{~cm}^{-3} \leq N_{d} \leq$ $10^{14} \mathrm{~cm}^{-3}$. Zero fixed oxide charge and interface trapped charge is assumed in the calculation of $V_{f b}$.

From Eq. 2.40 the current $I$ can be calculated, as a function of gate bias relative to the source, $V_{\text {gate }}$, drain bias relative to the source, $V_{d s}$, and backside (body-to-source) reverse bias, $-V_{b s}$. For a p-type substrate, reverse bias implies the substrate is at a negative bias relative to the source. Therefore, the backside reverse bias is negative. For long channel MOSFETs the source-to-drain spacing, or mask channel length $L_{m}$, is much larger than the depletion width under the gate, $w$. Under these conditions, the junction depth does not influence the results, and drain bias variations do not appreciably alter the electrical channel length $L$. The quasi-Fermi level $\phi_{F_{n}}$ as a function of band bending $\phi_{s}$ using Gauss's law, is given by

$$
\beta \phi_{F n}=\beta \phi_{F}-\ln \left\{\frac{\left(\beta V_{g a t e}-\beta \phi_{s}\right)^{2} / \alpha^{2}-\beta \phi_{s}}{\left(n_{i} / N_{A}\right)^{2}\left[e^{\left(\beta \phi_{s}\right)}-1\right]}\right\}
$$

Eq. 2.42 determines $\phi_{F n}$ in terms of $\phi_{s}$ at any point in the channel. As a result, the equation for the current and for the carrier density are functions of only the one variable, $\phi_{s}$, rather than both $\phi_{s}$ and $\phi_{F_{n}}$. In Fig. 2.10, $\beta \phi_{F n}+\beta \phi_{F}$ is plotted as a function of $\beta \phi_{s}$.

So far, $\beta \phi_{F_{n}}$ becomes infinity when the numerator of the logarithm vanishes, for example when $\beta \phi_{s}$ has the value $\beta \phi_{\text {sat }}$ which satisfies

$$
\left(\beta V_{\text {gate }}-\beta \phi_{\text {sat }}\right)^{2}=\alpha^{2}\left(\beta \phi_{\text {sat }}\right)
$$

Eq. 2.43 is usually referred as the pinch-off condition and the values of $\beta \phi_{s a t}$ also are shown in Fig. 2.10. At the source end of the channel, the quasi-Fermi level, $\phi_{F_{n}}$, is equal to that of the source itself,

$$
\phi_{F_{n}}=\phi_{F}(\text { source })=\phi_{F}+V_{b s}
$$




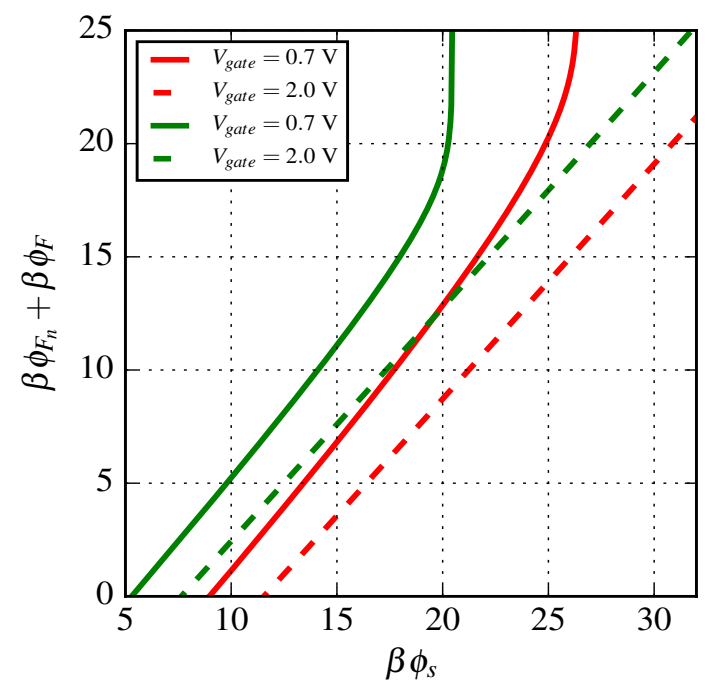

Figure 2.10: Electron quasi-Fermi level, $\beta \phi_{F_{n}}$ as a function of $\beta \phi_{S}$ for two $V_{g a t e}$ values, green color for $t_{o x}=250 \mathrm{~nm}, N_{d}=6.2 \cdot 10^{11} \mathrm{~cm}^{-3}$ and red color for $t_{o x}=700 \mathrm{~nm}, N_{d}=4.4 \cdot 10^{12} \mathrm{~cm}^{-3}$.

For $V_{b s}=0, \phi_{F n}=\phi_{F}$ because source and substrate are at equilibrium and the band bending at the source end of the channel is the same as the band bending in a MOS capacitor with gate bias $V_{\text {gate }}$. Similar at the drain end of the channel

$$
\phi_{F_{n}}=\phi_{F}(\text { drain })=\phi_{F}+V_{b s}+V_{d s} .
$$

The value of $V_{d s}$ at which $\phi_{s}$ becomes very nearly $\phi_{s a t}$, near the knees of the curves in Fig. 2.10, is called the saturation voltage, $V_{d s, s a t}$, defined as

$$
V_{d s, s a t}=\phi_{s a t}-\phi_{s 0}
$$

For values of $V_{d s}>V_{d s, s a t}$, the additional drain bias does not increase the band bending, but increases the electric field in the drain transition region, between the drain end of the channel and the drain. In addition, the size of this transition region increases with $V_{d s}$, causing a reduction of the channel length, $L$. This channel length is the distance between the source end of the channel and the drain end of the channel and is not the same as the metallurgical source-to-drain separation, $L_{m}$. For a long channel MOSFET, $L$, will not be very different from the source-to-drain separation.

Using the derivative of Eq. 2.42 as a function of $x$, the total charge in the inversion layer as

$$
q_{0} N_{I}=C_{o x}\left(V_{g}-\phi_{s}\right)-q_{0} N_{d} L_{D} \sqrt{2}\left(\beta \phi_{s}\right)^{1 / 2}
$$

and integrate Eq. 2.48, from the source end of the channel to the drain end of the channel as,

$$
\int_{0}^{L} I d x=-\int_{0}^{L}\left(W q_{0} \mu N_{I} \frac{d \phi_{F_{n}}}{d x}\right) d x
$$


the drain current, $I_{d}$, is calculated

$$
\begin{gathered}
I_{d}=-(W / L) \mu C_{o x} \beta^{-2}\left\{\left(1+\beta V_{g}\right)\left(\beta \phi_{s L}-\beta \phi_{s 0}\right)-(1 / 2)\left[\left(\beta \phi_{s L}\right)^{2}-\left(\beta \phi_{s 0}\right)^{2}\right]\right. \\
\left.-(2 / 3) \alpha\left[\left(\beta \phi_{s L}\right)^{3 / 2}-\left(\beta \phi_{s 0}\right)^{3 / 2}\right]+\alpha\left[\left(\beta \phi_{s L}\right)^{1 / 2}-\left(\beta \phi_{s 0}\right)^{1 / 2}\right]\right\} .
\end{gathered}
$$

For any given gate bias,

$$
V_{g}=V_{\text {gate }}+V_{b s}
$$

where $\beta \phi_{s 0}$ is found at the source end of the channel, Eq. 2.44, and $\beta \phi_{s L}$ is found at the drain end of the channel, Eq. 2.45. It is required that

$$
0 \leq \phi_{s L} \leq \phi_{\text {sat }}
$$

where $\phi_{s L}=0$ corresponds to $V_{d s}=0$ and $\phi_{s L}=\phi_{s a t}$ corresponds to an infinite $V_{d s}$, Fig. 2.10. To generate MOSFET characteristics using Eq. 2.49, the procedure is to choose $V_{b s}$ and $\phi_{s 0}$. Then, Eq. 2.42 and 2.44 determine $V_{g}$ as

$$
\beta V_{g}=\beta \phi_{s 0}+a\left\{\beta \phi_{s 0}+e^{\left(\beta \phi_{s 0}-2 \beta \phi_{F}-\beta V_{b s}\right)}\right\}^{1 / 2}
$$

In order to generate drain current characteristics as a function of drain bias for a given gate bias $V_{g}$, Eq. 2.42, must be solved for $\phi_{s 0}$ [28]. When $\phi_{s 0}$ is known, the current $I_{d}$ as a function of drain bias can be calculated using an allowed range of values of $\phi_{s L}$, Eq. 2.51. In addition, curves of current $I_{d}$ as a function of gate bias for a given drain bias also can be obtained. For chosen values of $V_{d s}, V_{b s}$, and $\phi_{s L}, V_{g}$ can be obtained from Eq. 2.52. With $V_{g}$ determined, $\phi_{s 0}$ can be calculated from Eq. 2.42. For $V_{b s}=0 \mathrm{~V}, V_{g}=V_{g a t e}$. With $\phi_{s 0}$ and $\phi_{s L}$ the current is determined from Eq. 2.64. The drain current in Eq. 2.64, includes both the drift and the diffusion component. Simpler formulas often are useful in limited bias ranges [29,38]. In Fig. 2.11 (a) is plotted normalized current $I_{d}$ as a function of drain-source voltage $V_{d s}$. At large gate voltage $V_{\text {gate }}$, the current $I_{d}$ increases almost linearly with $V_{d s}$ and this is the linear regime.

As $V_{d s}$ is increased further, the curves begin to drop below a straight-line dependence upon $V_{d s}$. The behavior is parabolic in $V_{d s}$. This parabolic regime, including the linear regime is called the triode region. For large enough $V_{d s}, V_{d s} \geq V_{d s, s a t}$, the current saturates at a value $I_{s a t}$. Increasing drain bias hardly affects the current in this region, the saturation region. In particular, at large $V_{\text {gate }}$ the silicon surface is strongly inverted. At low $V_{d s}$, the strong inversion region extends from source to drain, and the channel behaves just like a resistor, the current is proportional to $V_{d s}$ (linear region). However, at larger $V_{d s}$ enough current flows to cause an $I R$ voltage drop along the channel, lowering the quasi-Fermi level and reducing the carrier density. More current implies more $I R$ drop which implies higher channel resistance. So far, the larger the $V_{d s}$, the higher the resistance. More $V_{d s}$ is needed to increase the current, than when the channel resistance is fixed (triode 


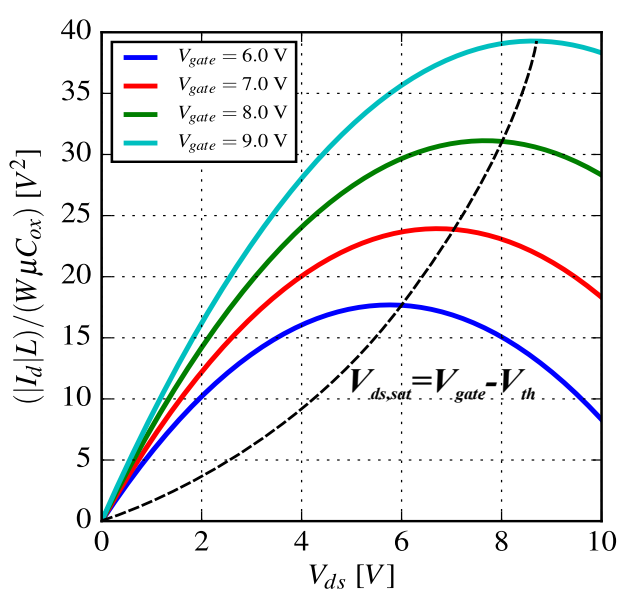

(a)

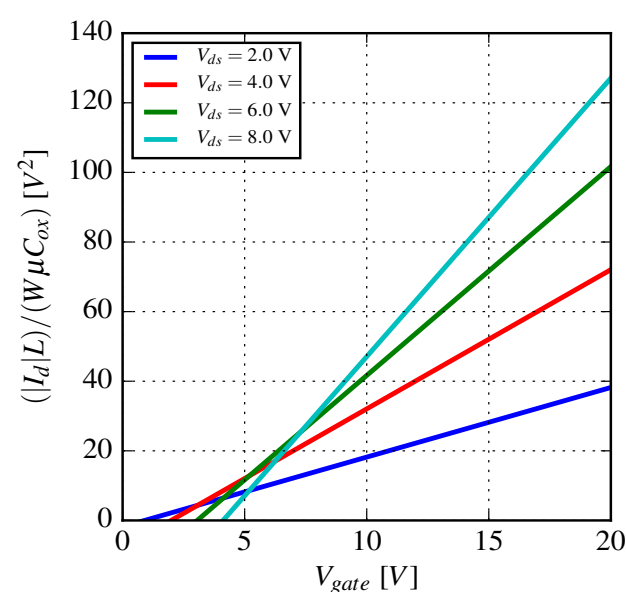

(b)

Figure 2.11: (a) Current $I_{d}$ as a function of drain-source voltage $V_{d s}$ with gate voltage $V_{\text {gate }}$ as parameter, and (b) Current $I_{d}$ as a function of gate voltage $V_{\text {gate }}$ with drain-source voltage $V_{d s}$ as parameter with $t_{o x}=700 \mathrm{~nm}$, $N_{d}=4.4 \cdot 10^{12} \mathrm{~cm}^{-3}, V_{f b}=\phi_{m s}$ and $V_{b s}=0$.

region). For $V_{d s}, V_{d s}>V_{d s, s a t}$, the carrier density at the drain end of the channel becomes so low that the band bending at this end of the channel no longer depends on the carrier density. The channel is pinched-off point $P$, Fig. 2.12, and any increase in $V_{d s}$ is simply dropped between the drain end of the channel and the drain, with virtually no effect on the current (saturation).

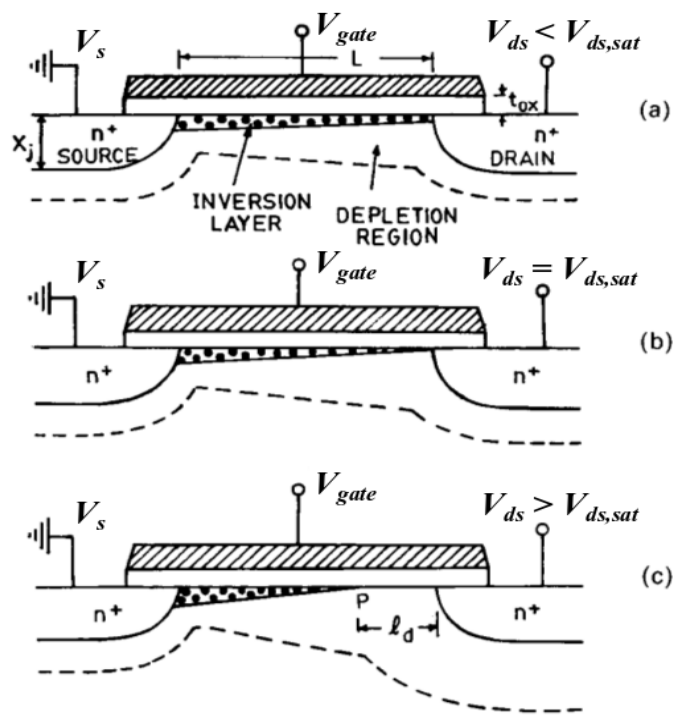

Figure 2.12: Schematic diagram of an n-MOSFET showing channel pinch-off as $V_{d s}$ is increased (a) an inversion layer connects the source and drain, $V_{d s}<V_{d s, s a t}$, (b) at the on set of saturation, the channel pinches off at the drain end, $V_{d s}=V_{d s, s a t}$ and (c) the pinch-off point $P$ moves towards the source. Figure taken from [29].

In Fig. 2.11 (a) the $V_{d s}$ beyond which saturation sets is indicated by a dashed line. In addition, in Fig. 2.11 (b) is presented the current $I_{d}$ as a function of $V_{\text {gate }}$ with $V_{d s}$ as parameter. For a fixed $V_{d s}$, current increases linearly with $V_{\text {gate }}$ provided $V_{\text {gate }}$ is not too low. 
Subthreshold region The subthreshold region corresponds to gate biases below $V_{T}$, for band bending less than $2 \phi_{F}+V_{b s}$. This band bending corresponds to weak inversion and allows $N_{I}$ to be simplified as in Eq. 2.22. In weak inversion, $N_{I}$, is small and $\phi_{s}$ is near saturation value $\phi_{s a t}$, as follows from Eq. 2.43. In addition, $\phi_{s}$ is almost the same everywhere in the channel. The change in $N_{I}$ between source $N_{I 0}$ and drain $N_{I L}$ is,

$$
N_{I 0}-N_{I L}=N_{d} L_{D}\left(n_{i} / N_{d}\right)^{2} e^{\left(\beta \phi_{s a t}\right)} e^{\left(-\beta V_{b s}\right)}\left[1-e^{\left(-\beta V_{d s}\right)}\right]\left(2 \beta \phi_{s a t}\right)^{-1 / 2}
$$

The band bending is constant along the channel in the subthreshold region, at a value $\phi_{\text {sat }}$. Therefore, there is no field to drive the current, and the current is due to diffusion. Diffusion current is driven by the gradient in carrier density, $d N_{I} / d x$. As the current must be the same at any point along the channel, $x, d N_{I} / d x$ must be constant and is obtained from

$$
\frac{d N_{I}}{d x}=\frac{N_{I L}-N_{I O}}{L}
$$

So far, in the subthreshold region

$$
\begin{aligned}
I_{d}=- & (W / L) \mu \beta^{-1} q_{0} N_{d} L_{D}\left(n_{i} / N_{d}\right)^{2} e^{\left(-\beta V_{b s}\right)} \\
& \times e^{\left(\beta \phi_{s a t}\right)}\left[1-e^{\left(-\beta V_{d s}\right)}\right]\left(2 \beta \phi_{s a t}\right)^{-1 / 2}
\end{aligned}
$$

where $\beta \phi_{s a t}$ is a function of $V_{g a t e}$ and $V_{b s}$

$$
\beta \phi_{s a t}=\beta V_{\text {gate }}+\beta V_{b s}+\alpha^{2} / 2-\alpha\left(\beta V_{\text {gate }}+\beta V_{b s}+\alpha^{2} / 4\right)^{1 / 2}
$$

Of course, the value of $\phi_{\text {sat }}$, affects $S$. In Fig. 2.13 (a) is presented $S$ as a function of $\alpha$ for two $\phi_{\text {sat }}$ values.

1) Near exponential dependence of $I_{d}$ upon gate bias [due to $\left.e^{\left(\beta \phi_{s a t}\right)}\right]$.

2) Independence of drain bias for $V_{d s} \gg k T / q_{0}$ [due to $\left.e^{\left(-\beta V_{d s}\right)} \ll 1\right)$ ].

3) More rapid reduction of $I_{d}$ with $V_{g}$ when body-to-source reverse bias is applied.

An important parameter characteristic of the subthreshold region is the gate-voltage swing S, corresponding to the reduction of the current by one decade. That is,

$$
S \equiv \ln 10\left\{\frac{d \ln I}{d V_{g}}\right\}^{-1}
$$

Using Eq. 2.55, $S$ can be evaluated for a long channel MOSFET

$$
S=\left(k T / q_{0}\right) \ln 10\left\{\frac{d \ln I}{d \beta \phi_{s a t}} \frac{d \beta \phi_{\text {sat }}}{d \beta V_{g}}\right\}^{-1}
$$




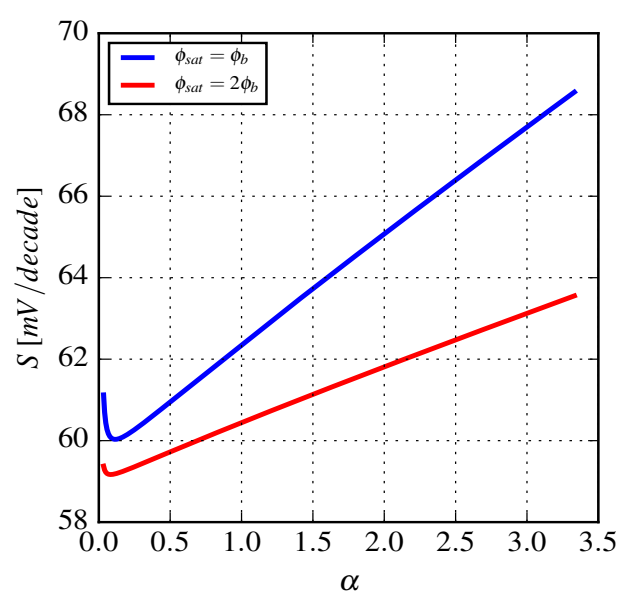

(a)

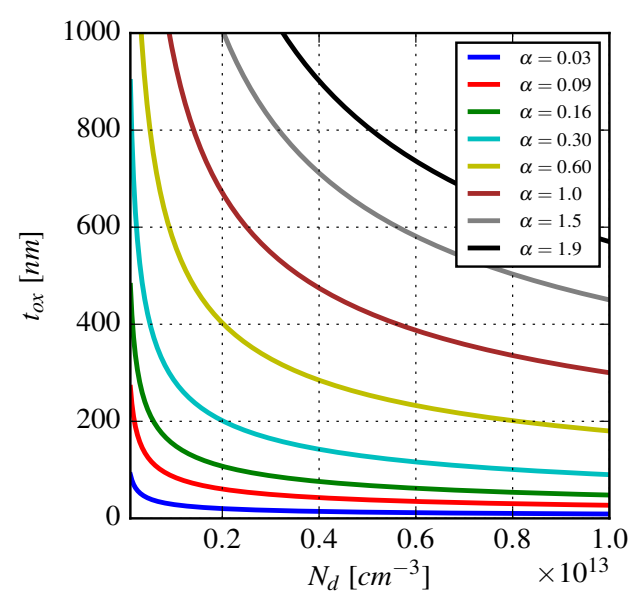

(b)

Figure 2.13: (a) Subthreshold swing $S$ required to reduce the subthreshold current one decade as a function of the parameter $\alpha$ and (b) Oxide thickness as a function of doping level for given $\alpha$ values.

$$
\begin{gathered}
C_{d e p}\left(\phi_{s a t}\right)=C_{f b}\left[2 \beta \phi_{s a t}\right]^{-1 / 2} \\
\frac{d \beta V_{g}}{d \beta \phi_{s a t}}=1+C_{d e p}\left(\phi_{s a t}\right) / C_{o x} \\
\frac{d \ln I}{d \beta \phi_{s a t}}=1-\left(2 / \alpha^{2}\right)\left[C_{d e p}\left(\phi_{s a t}\right) / C_{o x}\right]^{2} \\
S=\left(k T / q_{0}\right) \ln 10\left[1+C_{d e p}\left(\phi_{s a t}\right) / C_{o x}\right] /\left\{1-\left(2 / \alpha^{2}\right)\left[C_{d e p}\left(\phi_{s a t}\right) / C_{o x}\right]^{2}\right\}
\end{gathered}
$$

Triode region This region corresponds to gate biases well above $V_{t h}$ and to drain biases below $V_{d s, s a t}$. In this regime, Fig. 2.10, shows that $\phi_{s}$ and $\phi_{F n}$ are almost linearly related with unity slope. The quasi-Fermi level $\phi_{F_{n}}$ changes by an amount $V_{d s}$ on going from source to drain, so

$$
\phi_{s L}=\phi_{s 0}+V_{d s}
$$

Using Eq. 2.63 and assuming $\beta \phi_{s} \gg 1$ the current $I_{d}$ becomes

$$
\begin{array}{r}
I_{d}=-(W / L) \mu C_{o x}\left\{\left(V_{g}-\phi_{s 0}-V_{d s} / 2\right) V_{d s}\right. \\
\left.-(2 / 3) \alpha \beta^{-1 / 2}\left[\left(\beta \phi_{s 0}+V_{d s}\right)^{3 / 2}-\left(\beta \phi_{s 0}\right)^{3 / 2}\right]\right\} .
\end{array}
$$


At low drain biases, the $\left(\frac{3}{2}\right)$ power terms can be expanded in a Taylor series

$$
\left(\phi_{s 0}+V_{d s}\right)^{3 / 2}-\phi_{s 0}^{3 / 2}=\left(\frac{3}{2}\right)\left(\phi_{s 0}\right)^{1 / 2} V_{d s}+\left(\frac{3}{2}\right)\left(\frac{1}{2}\right)\left(\phi_{s 0}\right)^{-1 / 2} V_{d s}^{2} / 2
$$

So far, using Eq. 2.36 for $V_{t h}$, Eq. 2.64 is written in its most commonly used form

$$
I_{d}=-(W / L) \mu C_{o x}\left\{V_{g}-V_{t h}-\left(\frac{1}{2}\right)\left[1+(\alpha / 2)\left(\beta \phi_{s 0}\right)^{-1 / 2}\right] V_{d s}\right\} V_{d s}
$$

This form is the basis of the experimental determination of threshold voltage. The current $I_{d}$ is plotted as a function of $V_{\text {gate }}$ at low value of $V_{d s}$. If $\mu$ is independent of $V_{g}$, according to Eq. 2.66, such a plot is a straight line with intercept at $V_{g}=V_{g 0}$ where

$$
V_{g 0}=V_{t h}+\left(\frac{1}{2}\right)\left[1+(\alpha / 2)\left(\beta \phi_{s 0}\right)^{-1 / 2}\right] V_{d s}
$$

Therefore, $V_{t h}$ can be determined from the intercept $V_{g 0}$, using the known $V_{d s}, \alpha$ and $\beta \phi_{s 0} \approx 2 \beta \phi_{b}$. In practice, this procedure is not straightforward because no straight-line region of $I_{d}$ as a function of $V_{g}$ is observed. The mobility of the carriers in the inversion layer varies with $V_{g}$. So far, the current $I_{d}$ as a function of $V_{g}$ curves change from a subthreshold behavior to a sublinear behavior as $V_{g}$ increases. A straight line is fitted to the $I_{d}\left(V_{g}\right)$ curve and the fitted line has the intercept $V_{g 0}$. The slope of the line fitted through the inflection point is equal to $(W / L) \mu C_{o x}$. Because $(W / L)$ and $C_{o x}$ can be measured independently, the slope determines the mobility. However, a theoretical treatment of the gate voltage dependence of mobility should be included in Eq. 2.66. The position of the inflection point is affected from the fixed oxide charge and the interface trapped charge, whenever these charge densities per unit area are higher than $10^{10} \mathrm{~cm}^{-2}$. Above an interface charge density of $10^{10} \mathrm{~cm}^{-2}$, the spatially nonuniform interface charge introduces a nonuniform inversion layer carrier density, at gate voltage near or below the threshold voltage.

Saturation region At $V_{g}$ values well above threshold and for $V_{d s} \gg V_{d s, s a t}, \beta \phi_{s L}=\beta \phi_{s a t}$. In this region, the current $I_{d}$ has saturated and is independent of $V_{d s}$. This current, $I_{d, s a t}$, is given approximately by

$$
I_{d, s a t}=m(W / L) \mu C_{o x}\left(V_{g}-V_{t h, s a t}\right)^{2}
$$

and the threshold voltage, $V_{t h, s a t}$ extracted from the intercept of $\sqrt{I_{d, s a t}}$ as a function of $V_{g}$ is given by

$$
V_{t h, s a t}=V_{f b}+\phi_{s 0}+\beta^{-1} \alpha\left(\beta \phi_{s 0}\right)^{1 / 2}
$$

where $\phi_{s 0}$ is the band bending at the source end of long channel region. The slope parameter, $m$, is given by

$$
1 / m=2\left\{1+\alpha /\left[\left(\beta \phi_{s a t}\right)^{1 / 2}+\left(\beta \phi_{s 0}\right)^{1 / 2}\right]\right\}
$$


Effective mobility The carrier inversion layer mobility values for electrons and holes are lower than the bulk mobility values. The carriers in the channel undergo scattering by the charges at the surface boundary and by surface roughness, in addition to the scattering with the crystal lattice and ionized impurity atoms. In particular, the carrier mobility of a MOSFET is a strong function of the properties of the $\mathrm{Si}_{-} \mathrm{SiO}_{2}$ interface and is strongly influenced by processing techniques. The carriers in the channel move under the influence of the normal electric field $E_{y}$ and the lateral electric field $E_{x}$ due to $V_{g a t e}$ and $V_{d s}$ respectively. The scattering increases with increasing fields. The normal field $E_{y}$ accelerates the charge carriers towards the surface causing carriers to scatter more frequently than in the absence of $V_{\text {gate }}$. The lateral field $E_{x}$ causes charge carriers to move faster, so at high $V_{d s}$ the carriers become velocity saturated. Clearly $\mu$ is not constant and depends on both $E_{y}$ and $E_{x}$, which is in contrast to the earlier assumption of constant $\mu$ and taken outside the integral in Eq. 2.48. In order to avoid complications, an effective mobility $\mu_{e f f}$ is defined as the average mobility of the carriers

$$
\mu_{e f f}=\frac{\int_{0}^{L} \mu_{s}(x, y) n(x, y) d x}{\int_{0}^{L} n(x, y) d x} .
$$

where $\mu_{s}(x, y)$ is the surface channel mobility and $n(x, y)$ is the channel carrier density. In general, at least three different scattering mechanisms, as shown in Fig. 2.14, have been proposed to account for the mobility behavior in the region where the gate voltage is above threshold [43]:

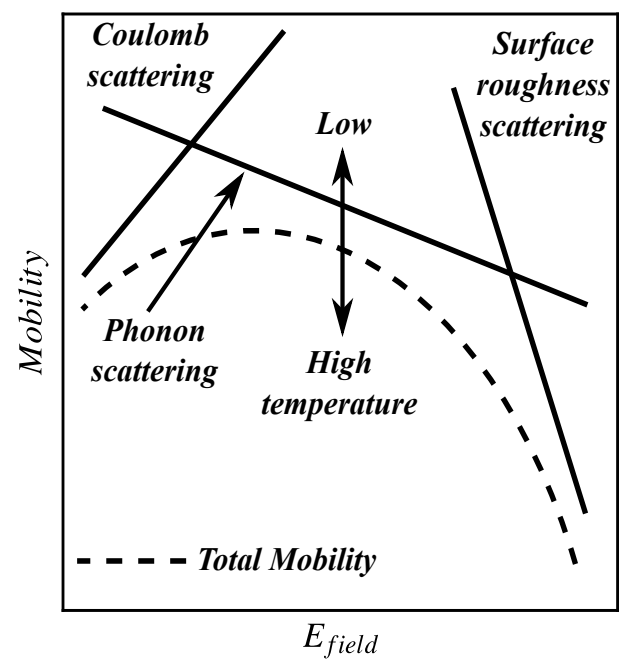

Figure 2.14: Schematic diagram of $E_{\text {field }}$ dependence of mobility in inversion layer by three dominant scattering mechanisms [44].

1) Phonon scattering due to the various modes of lattice vibration including surface acoustic phonons and optical phonons. Phonon scattering is important at room temperature and can be ignored at very low temperatures [45].

2) Coulomb scattering due to charged centers, including fixed oxide charge, interface-state charge and localized charge due to ionized impurities. The effects of Coulomb scattering are important for lightly inverted surfaces. High surface-charge densities or substrate doping concentrations imply increased Coulomb scattering. This type of scattering becomes less effective for a heavily inverted surface because of carrier screening [46]. 
3) Surface-roughness scattering by the deviation of the interface from an ideal plane. This type of scattering is important under strong inversion conditions because the strength of the interaction is governed by the distance of the carriers from the surface; the closer the carriers are to the surface, the stronger the scattering due to surface roughness will be [47-49].

The relative importance of these scattering mechanisms depends on the operating temperature and the strength of the surface electric field. At low temperatures, the mobility is determined by the combined effects of Coulomb scattering in the low-field region, and by surface roughness in the high-field region. At room temperature, the mobility is governed by Coulomb scattering due to charged centers and phonon scattering in the low-field region. It is dominated by surface roughness and phonon scattering under strong inversion [43].

To develop a theoretical model for $\mu_{e f f}$ is not easy, because separation of the contributions of the various scattering mechanisms is difficult due to the many parameters involved. Theoretical analyses are complicated by the confinement of the channel region to a very small thickness in a potential well at the silicon surface. The theory is further complicated by quantum effects which play an important role [50], and because surface roughness requires further investigation [51,52]. The prediction of the effective mobility relies on experimental data and empirical equations [43,53]. For example empirical relationship of the following form,

$$
\mu_{e f f}=\mu_{0}\left(\frac{E_{0}}{E_{e f f}}\right)^{v}
$$

where $\mu_{0}$ is the maximum extracted value of the mobility at a given doping concentration, is called the low field surface mobility. $E_{0}$ is the critical electric field below which $\mu_{e f f}=\mu_{0}$ and above which $\mu_{e f f}$ begins to decrease and $v$ is an empirical constant. $E_{\text {eff }}$ is the effective electric field at the $\mathrm{Si}_{-} \mathrm{SiO}_{2}$ interface. An increase in $E_{\text {eff }}$ causes carriers to be drawn closer to the interface, so surface scattering increases and hence lowers the mobility. It has been observed that the mobility is independent of the gate oxide thickness, provided that the $\mathrm{Si}_{-} \mathrm{SiO}_{2}$ interface is of good quality (oxide charge density less than $10^{10} \mathrm{~cm}^{-2}$ ) and the channel inversion charge is properly calculated. So far, $\mu_{e f f}$ is more a function of the $\mathrm{Si}_{-} \mathrm{SiO}_{2}$ interface than device parameters such as oxide thickness or doping concentration. A similar mobility model which fits experimental data at low $V_{d s}$ for both p- and n-MOSFETs, is of the form

$$
\mu_{e f f}=\frac{\mu_{0}}{1+\alpha_{\theta} E_{e f f}}
$$

where $\alpha_{\theta}$ is called the scattering constant. The $E_{\text {eff }}$ is related to the bulk depletion charge and to the inversion charge. Assuming a constant channel electric field in the lateral direction Eq. 2.73 can be written simply

$$
\mu_{e f f}=\frac{\mu_{0}}{1+\theta\left(V_{\text {gate }}-V_{t h}\right)+\theta_{b} V_{b s}}
$$

where 


$$
\theta=\frac{\alpha_{\theta} \eta C_{o x}}{\varepsilon_{0} \varepsilon_{S i}}
$$

is called the mobility degradation coefficient and $\eta$ is a weighting factor equal to $1 / 2$ and $1 / 3$ for electrons and holes respectively. The effect of $V_{b s}$ on the mobility is simply that the surface field is increased at the onset of inversion because of the increase in bulk charge. This is equivalent to an increase in substrate impurity concentration, except that there is no variation in the impurity concentration inside the inversion layer. So far, if the effective (average) gate field is used, the mobility curve of $V_{b s}=0$ is still applicable [43, 53]. 



\section{MOSFET structures and operation}

The effective channel length $L_{e f f}$ and width $W_{\text {eff }}$ of a MOSFET are important device parameters in order to model the structure using TCAD simulations [6]. Usually there are differences between the mask defined and the physical dimensions. In this chapter are presented how these differences influence the mobility $\mu$ at the $\mathrm{Si}_{-} \mathrm{SiO}_{2}$ interface and the threshold voltage $V_{t h}$.

\subsection{Structures}

The investigated test structures are shown in Figs. 3.1 and 3.2. In Fig. 3.1 (a) and (b) is shown the cross section and top view of the rectangular p-MOSFETs $<100>$. The structures are build on high-ohmic $\mathrm{n}-\mathrm{Si}$ with a common gate for three different channel lengths.

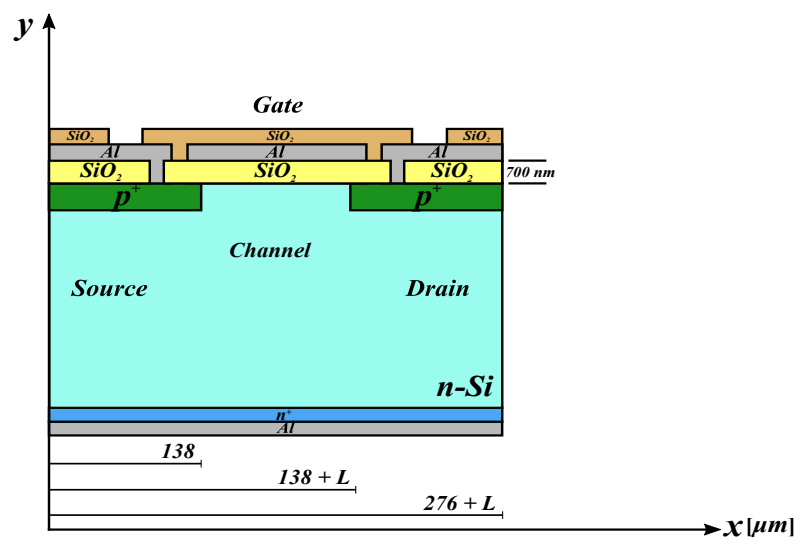

(a)

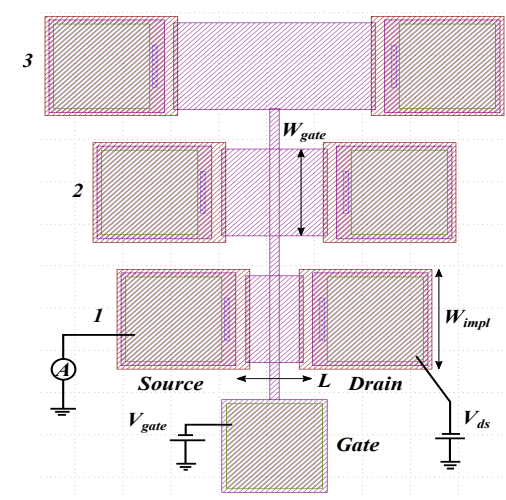

(b)

Figure 3.1: (a) Cross section and (b) measurement setup top view of the rectangular p-MOSFET $<100>$ for the three different $L_{m}$ channel lengths.

The defined mask channel lengths $L_{m}$ are 52,102, $202 \mu \mathrm{m}$ and the gate width $W_{\text {gate }}=103 \mu \mathrm{m}$. For the circular structure, the $W / L$ ratio

$$
\frac{W}{L}=\frac{2 \pi}{\ln \frac{r_{\text {drain }}}{r_{\text {source }}}}
$$




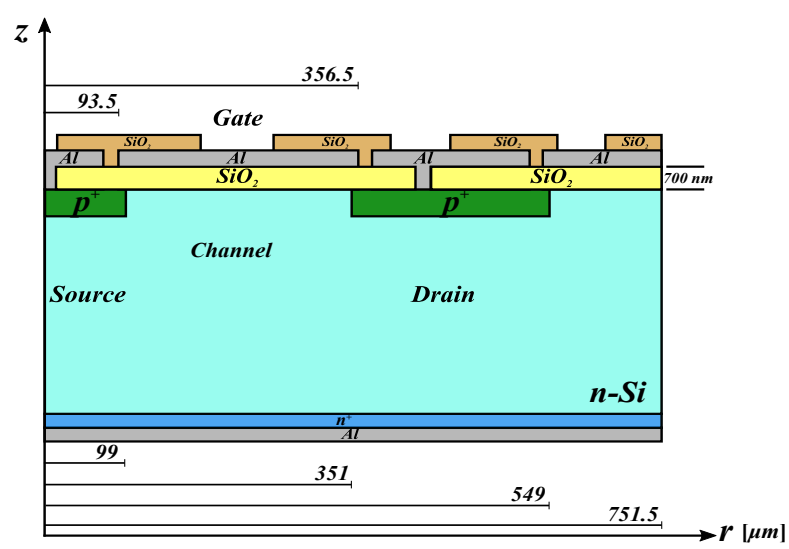

(a)

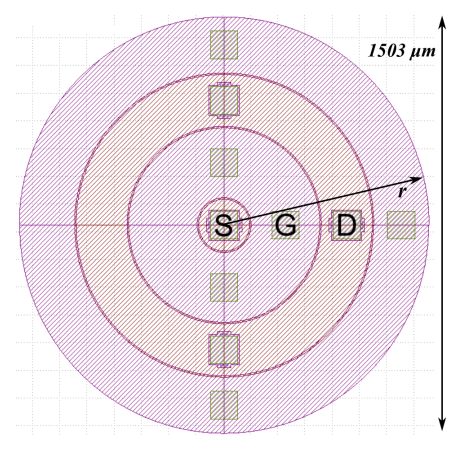

(b)

Figure 3.2: (a) Cross section and (b) top view of the circular p-MOSFET $<100>$.

is derived using the radius of the source implant, $r_{\text {source }}$, and the inner radius of the drain implant $r_{\text {drain }}$. In Fig. 3.2 (a) and (b) is shown the cross section and top view of the circular structure. The mask channel length is $252 \mu \mathrm{m}$ with $r_{\text {source }}=99$ and inner $r_{\text {drain }}=351 \mu \mathrm{m}$.

\subsubsection{Drain-source current characteristics}

The drain-source current characteristics of the above circular and rectangular p-MOSFETs are presented in Fig. 3.3. The devices are operated in the enhancement mode, no conducting channel between source and drain at zero gate voltage $[29,54]$. They are normally-off.

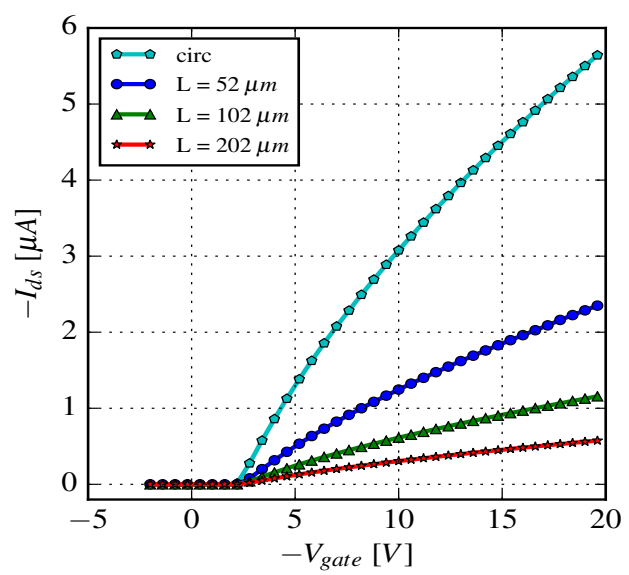

(a)

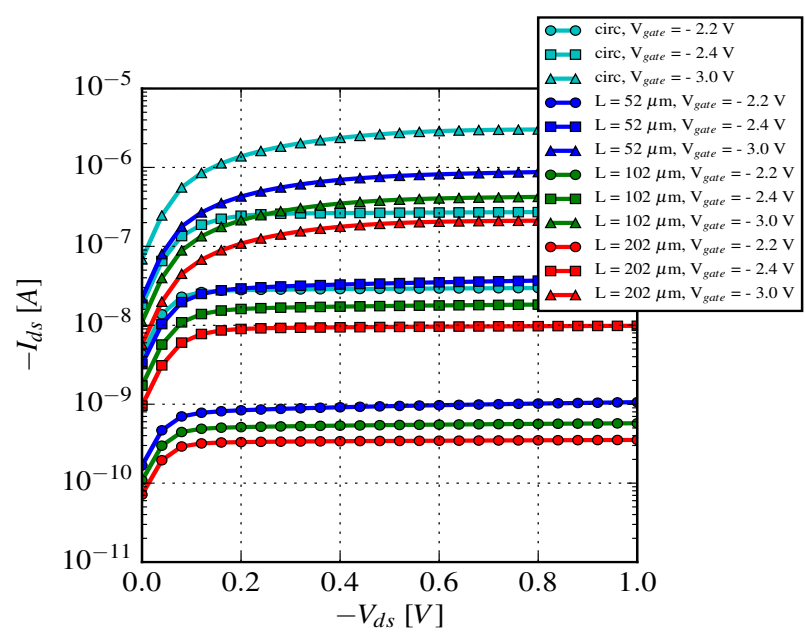

(b)

Figure 3.3: (a) $I_{d s}$ as a function of $V_{g a t e}$ in the linear region, $V_{d s}=-50 \mathrm{mV}$, and (b) $I_{d s}$ as a function of $V_{d s}$ for three $V_{\text {gate }}$ values of the circular and the three rectangular p-MOSFETs $<100>$. 
As shown in Fig. 3.3 (a), the drain-source current $I_{d s}$ as a function of gate voltage $V_{\text {gate }}$ in the linear region, for drain-source voltage $V_{d s}=-50 \mathrm{mV}$, depends on the channel length. For the rectangular design, the smaller the length, the higher $I_{d s}$ at the same $V_{\text {gate }}$ value. For the circular design, the $W / L \approx 4.96$ is higher compared to the rectangular $W / L$, so higher $I_{d s}$ is expected. Similar effects are presented in Fig. 3.3 (b) for the $I_{d s}$ as a function of $V_{d s}$ for three $V_{g a t e}$ values $-2.2,-2.4$, and $-3.0 \mathrm{~V}$, as also the saturation current depends on the $W / L$ ratio. After normalization to $L / W$ ratio, in Fig. 3.4 (a) the $I_{d s}\left(V_{\text {gate }}\right)$ curves do not show a good agreement. In addition, the normalization difference is shown in Fig. 3.4 (b) for the $I_{d s}\left(V_{d s}\right)$ curves.

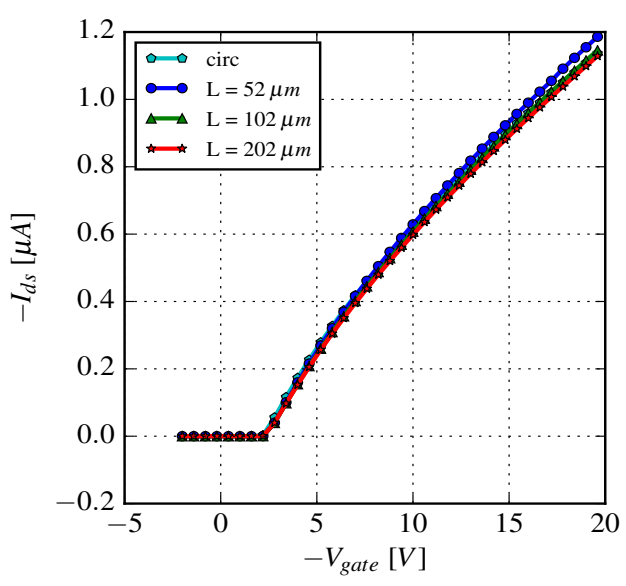

(a)

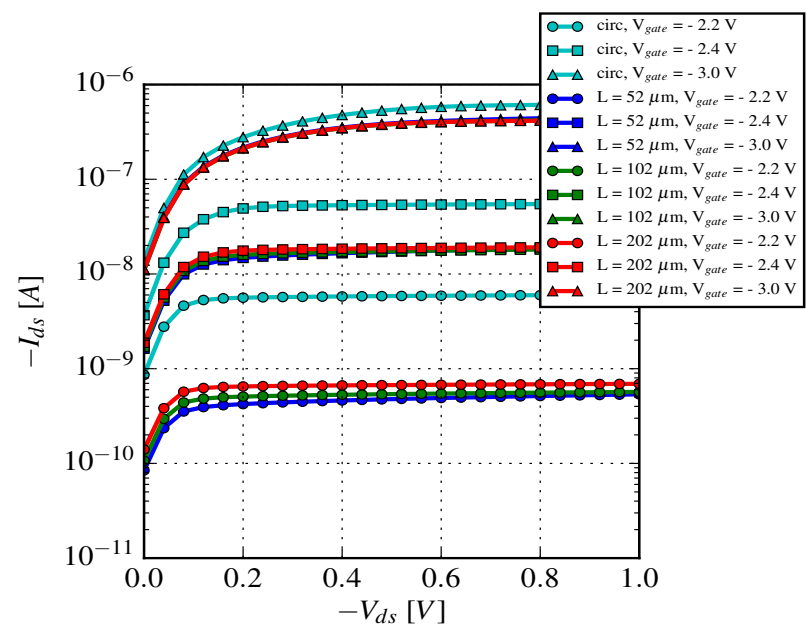

(b)

Figure 3.4: (a) $I_{d s}$ as a function of $V_{\text {gate }}$ in the linear region and (b) $I_{d s}$ as a function of $V_{d s}$ for three $V_{\text {gate }}$ values normalized to $L / W$, of the circular and the three rectangular p-MOSFETs $<100>$.

For the circular design, the higher $I_{d s}\left(V_{d s}\right)$ for $V_{\text {gate }}=-2.2 \mathrm{~V}$ is related to the difference in $V_{t h}$, as for $V_{\text {gate }}=$ $-3.0 \mathrm{~V}$ the agreement with the rectangular design has been improved. In order to understand the above effects, a further investigation is necessary of the effective channel length, mobility, gate-bias dependence and parasitic series resistance.

\subsection{Length and width definitions}

According to Refs. [55-63], many methods have been developed to measure the effective channel length, $L_{e f f}$, of a MOSFET. Most of them are based on the device's current voltage characteristics at low drain bias above threshold [58]. The basic assumptions of nearly every channel length extraction method are: channel length independent mobility and gate voltage independent series resistance [60]. In the present analysis is used the De La Moneda method [56].

\subsubsection{Series resistance and channel length}

The MOSFET source/drain series resistance and the $L_{e f f}$ or $W_{\text {eff }}$ are determined from the $I_{d s}\left(V_{\text {gate }}\right)$ for low $V_{d s}$ in the linear region. The resistance between source and drain consists of source resistance, channel 
resistance, drain resistance and contact resistances. The source resistance $R_{S}$ and drain resistance $R_{d}$ are shown in Fig. 3.5 (a).

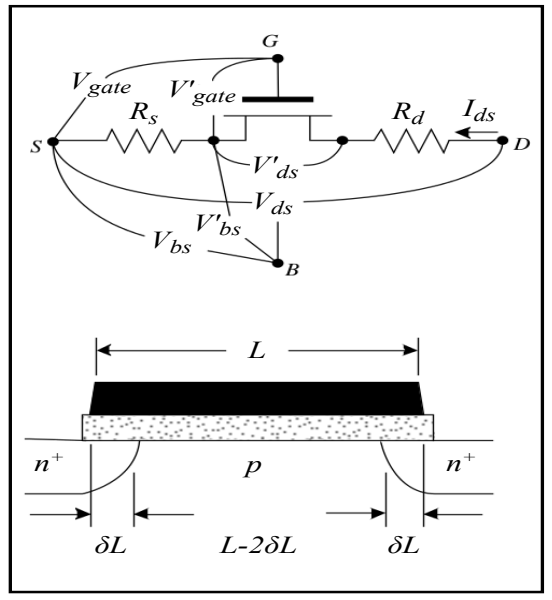

(a)

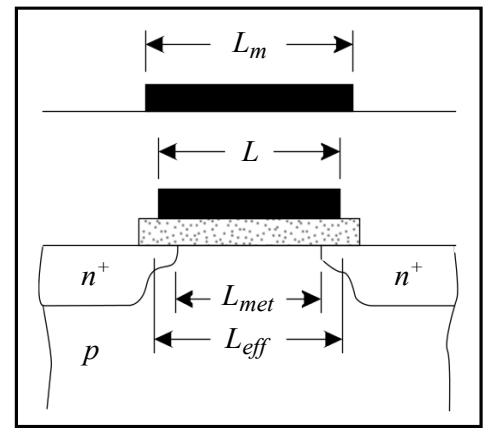

(b)

Figure 3.5: (a) MOSFET source and drain resistances and device cross section showing the actual gate length $L$ and $L_{e f f}=L-\Delta L$ with $\Delta L=2 \delta L$ and (b) Various MOSFET gate lengths: mask length $L_{m}$, physical gate length $L$, metallurgical $L_{m e t}$ and effective channel lengths $L_{e f f}$. Figures adapted from [55].

They are due to the source and drain contact resistance, the sheet resistance of the source and drain, the spreading resistance at the transition from the source diffusion to the channel, and any additional "wire" resistance. The channel resistance is contained in the MOSFET symbol and is not shown. The $L_{e f f}$ differs from the mask defined length, due to source and drain junction formation under the gate, as shown in Fig. 3.5 (b). $L_{m}$ is the mask defined gate length, $L$ the physical gate length, $L_{m e t}$ the metallurgical channel length (distance between source and drain), and $L_{e f f}$ the effective channel length. $L_{e f f}$ is not always equal to $L_{m e t}$. In general $L_{e f f}$ can be defined as the channel length that gives good agreement between measurement and theory when it is substituted into appropriate model equation [55]. Neglecting the body effect of the ionized bulk charge in the MOSFET space charge region, the MOSFET current voltage equation, valid for low drain voltage, can be written as

$$
I_{d s}=\mu_{e f f} C_{o x} \frac{W_{e f f}}{L_{e f f}}\left(V_{g a t e}^{\prime}-V_{t h}-\frac{1}{2} V_{d s}^{\prime}\right) V_{d s}^{\prime}
$$

where $W_{\text {eff }}=W_{\text {gate }}-\Delta W_{\text {gate }}, L_{\text {eff }}=L-\Delta L, V_{\text {th }}$ is the threshold voltage, $V_{g s}^{\prime}$ and $V_{d s}^{\prime}$ are defined in Fig. 3.5 (a), $W_{\text {gate }}$ is the gate width, $L$ the gate length, $C_{o x}$ the oxide capacitance per unit area and $\mu_{e f f}$ the effective mobility. $W$ and $L$ usually refer to the mask dimensions. With $V_{\text {gate }}=V_{\text {gate }}^{\prime}+I_{d s} R_{s}$ and $V_{d s}=V_{d s}^{\prime}+I_{d s}\left(R_{s}+\right.$ $R_{d}$ ), Eq. 3.2 can be written as

$$
I_{d s}=\mu_{e f f} C_{o x} \frac{W_{e f f}}{L_{e f f}}\left(V_{g a t e}-V_{t h}-\frac{1}{2} V_{d s}\right)\left(V_{d s}-I_{d s} R_{s d}\right)
$$


if $R_{s}=R_{d}=R_{s d} / 2$, where $R_{s d}=R_{s}+R_{d}$. For low drain-source voltage $\left(V_{d s} \approx 50-100 \mathrm{mV}\right)$ the device is operated in the linear region. For the device biased in strong inversion, with $\left(V_{\text {gate }}-V_{t h}\right) \gg V_{d s}$, Eq. 3.3 becomes

$$
I_{d s}=\mu_{e f f} C_{o x} \frac{W_{e f f}}{L_{e f f}}\left(V_{g a t e}-V_{t h}\right)\left(V_{d s}-I_{d s} R_{s d}\right)
$$

which can be written as

$$
I_{d s}=\frac{\mu_{e f f} C_{o x} W_{e f f}\left(V_{g a t e}-V_{t h}\right) V_{d s}}{(L-\Delta L)+\mu_{e f f} C_{o x} W_{e f f}\left(V_{g a t e}-V_{t h}\right) R_{s d}} .
$$

From Eq. 3.5 the entire channel resistance $R_{m}$ can be written as

$$
R_{m}=R_{c h}+R_{s d}=\frac{L-\Delta L}{\mu_{e f f} C_{o x} W_{e f f}\left(V_{g a t e}-V_{t h}\right)}+R_{s d}
$$

where $R_{c h}$ is the channel resistance and $R_{s d}$ the source/drain resistance. The mobility $\mu_{e f f}$ can be written as

$$
\mu_{\text {eff }}=\frac{\mu_{0}}{1+\theta\left(V_{\text {gate }}^{\prime}-V_{\text {th }}\right)}=\frac{\mu_{0}}{1+\left(V_{\text {gate }}-I_{d s} R_{s}-V_{\text {th }}\right) / V_{1 / 2}} \approx \frac{\mu_{0}}{1+\left(V_{\text {gate }}-V_{\text {th }}\right) / V_{1 / 2}}
$$

where $\theta=1 / V_{1 / 2}$ with $V_{1 / 2}$ the gate voltage at which $\mu_{e f f}=\mu_{0} / 2 . R_{m}$ is derived in Eq. 3.8, with slope $m$ and intercept $R_{m i}$.

$$
\begin{gathered}
R_{m}=\frac{L-\Delta L}{\mu_{0} C_{o x} W_{e f f}\left(\left|V_{\text {gate }}-V_{t h}\right|\right)}+\frac{\left|V_{1 / 2}^{-1}\right|(L-\Delta L)}{\mu_{0} C_{o x} W_{\text {eff }}}+R_{\text {sd }} \\
m=\frac{L-\Delta L}{\mu_{0} C_{o x} W_{\text {eff }}} \\
R_{m i}=\frac{\left|V_{1 / 2}^{-1}\right|(L-\Delta L)}{\mu_{0} C_{o x} W_{e f f}}+R_{s d}=\left|V_{1 / 2}^{-1}\right| m+R_{\text {sd }}
\end{gathered}
$$

Using Eq. 3.2, the parameters $\mu_{0}, V_{1 / 2}$ and $V_{t h}$ are extracted. The measurements and the fit results for the circular and the rectangular FZ320 p-MOSFET $<100>$ are presented in Fig. 3.6 (a) and Table 3.1, for electric field $E_{\text {field }}$ at the $\mathrm{Si}_{-} \mathrm{SiO}_{2}$ interface $\approx 250 \mathrm{kV} / \mathrm{cm}$. Differences are observed for the mobility parameters $\mu_{0}$ and $V_{1 / 2}$ between the circular and rectangular design. Smaller differences are observed for the $V_{t h}$ parameter. The effects of the parasitic series resistance due to the existence of uncertain source-drain diffusion layer edges should be taken into account. The $\Delta L, R_{s d}, \mu_{0}$ and $V_{1 / 2}$ are extracted using Eq. 3.8, according to the method presented in Ref. [56]. First $R_{m}$ is plotted against $1 /\left(V_{\text {gate }}-V_{t h}\right)$ as shown in Fig. 3.6 (b).

The slope of this plot is $m$ defined in Eq. 3.9 and the intercept on the $R_{m}$ axis is defined in Eq. 3.10. The slope $m$ is plotted against $L$, presented in Fig. 3.7 (a). This plot has a slope of $1 / W_{e f f} \mu_{0} C_{o x}$ and an intercept 


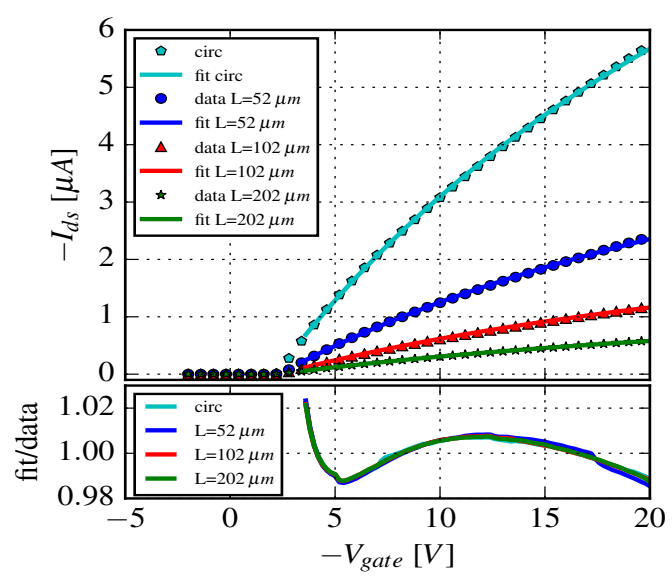

(a)

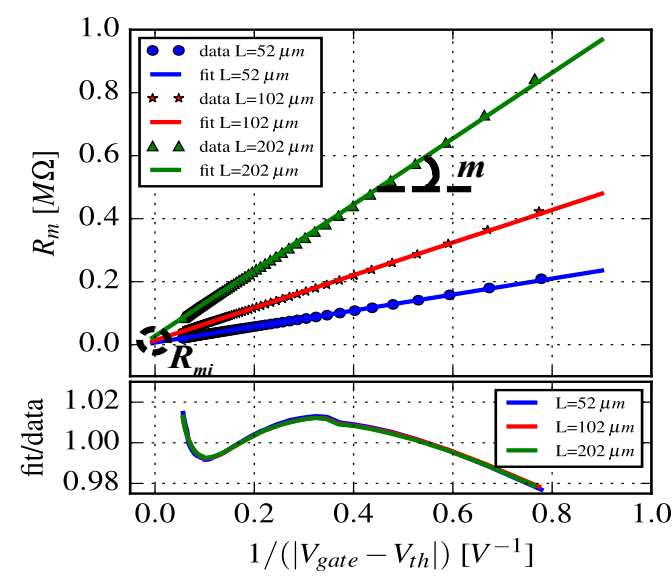

(b)

Figure 3.6: (a) $I_{d s}$ as a function of $V_{\text {gate }}$ and the parametrization fit used to determine the threshold voltage $V_{t h}$ and the hole mobility of the circular and rectangular p-MOSFETs $<100>$ and (b) $R_{m}$ as a function of $1 /\left(\left|V_{\text {gate }}-V_{t h}\right|\right)$ and the parametrization fit used to determine the slope $m$ and the intercept $R_{m i}$ of the rectangular p-MOSFETs $<100>$.

Table 3.1: Comparison of the extracted parameters between the circular and the rectangular FZ320 pMOSFET $<100>$ before correction for $\Delta L$.

\begin{tabular}{lllll}
\hline $\mathrm{p}-\mathrm{MOSFET}$ & $\mu_{0}\left[\mathrm{~cm}^{2} /(\mathrm{Vs})\right]$ & $V_{1 / 2}[\mathrm{~V}]$ & $V_{\text {th }}[\mathrm{V}]$ & $E_{\text {field }}[\mathrm{kV} / \mathrm{cm}]$ \\
\hline circ-FZ320, $\mathrm{L}=252 \mu \mathrm{m}$ & 394.4 & 34.2 & 2.11 & 260 \\
rec-FZ320, $\mathrm{L}=52 \mu \mathrm{m}$ & 403.0 & 37.0 & 2.32 & 250 \\
rec-FZ320, $\mathrm{L}=102 \mu \mathrm{m}$ & 387.8 & 37.7 & 2.31 & 250 \\
rec-FZ320, $\mathrm{L}=202 \mu \mathrm{m}$ & 380.4 & 38.1 & 2.29 & 250 \\
\hline
\end{tabular}

on the $L$ axis of $\Delta L$, allowing $\mu_{0}$ and $\Delta L$ to be determined. In addition, $R_{m i}$ is plotted as a function of $m$, and $V_{1 / 2}^{-1}$ is determined from the slope and $R_{s d}$ from the intercept on the $R_{m i}$ axis. Two structures with different channel lengths suffice for these measurements. The channel lengths should be selected to minimize the error in $\Delta L$ associated with the extrapolation of the $m$ versus $L$ plot. Errors in $\Delta L$ are minimized by choosing channel lengths that differ by about a factor of ten.

Table 3.2: Comparison of the extracted parameters between the rectangular FZ320 and M200 p-MOSFET $<100>$ after correction for $\Delta L$.

\begin{tabular}{llllll}
\hline p-MOSFET & $\mu_{0}\left[\mathrm{~cm}^{2} /(\mathrm{Vs})\right]$ & $V_{1 / 2}[\mathrm{~V}]$ & $\Delta L[\mu \mathrm{m}]$ & $\delta L[\mu \mathrm{m}]$ & $R_{s d}[\Omega]$ \\
\hline rec-FZ320 & $373.2 \pm 0.3$ & $38.5 \pm 0.1$ & $3.86 \pm 0.05$ & $1.93 \pm 0.03$ & $269 \pm 26$ \\
rec-M200 & $373.1 \pm 0.2$ & $36.8 \pm 0.1$ & $3.42 \pm 0.05$ & $1.71 \pm 0.03$ & $125 \pm 27$ \\
\hline
\end{tabular}

The method has been applied on Float-Zone with $320 \mu \mathrm{m}$ (FZ320) and Magnetic Czochralski with $200 \mu \mathrm{m}$ (M200) physical silicon wafer thickness, respectively. The data and the linear fits are shown in Fig. 3.8 and the results in Table 3.2 .

The mobility parameter $\mu_{0}$, which is related to Coulomb scattering effects, for the FZ320 and M200 pMOSFET is approximately the same. Small difference is observed for the $V_{1 / 2}$ which is related to scattering due to surface roughness. In addition, differences are observed for the $\delta L$ and $R_{s d}$ parameters. The $\delta L$ parameter has been extracted in the expected range, assuming a junction depth $\chi_{j}$ of the source/drain regions 


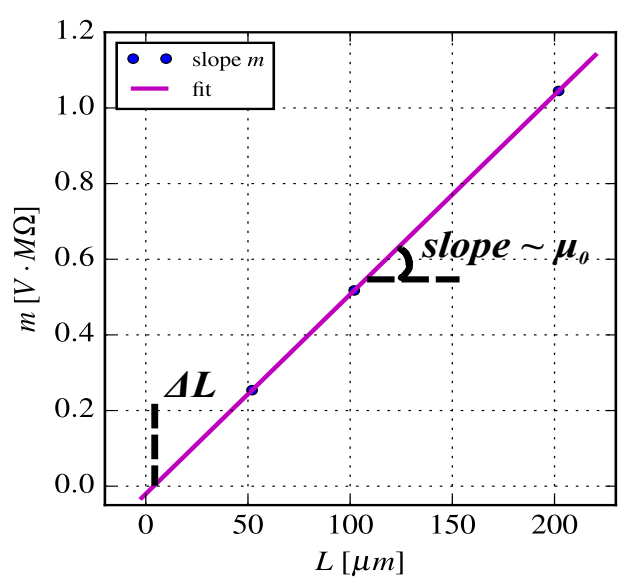

(a)

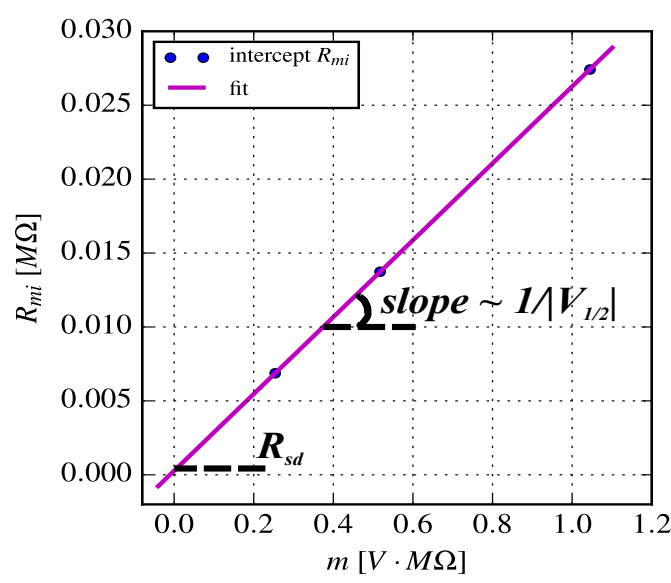

(b)

Figure 3.7: (a) Slope $m$ as a function of $L$ and (b) Intercept $R_{m i}$ as a function of $m$.

around $2.4 \mu \mathrm{m}, \delta L$ is approximately $0.8 \chi_{j}$. The $W_{\text {eff }}$ could be determined in the same way as indicated above for the $L_{e f f}$, if at least two structures with different channel widths are available for these measurements, Fig. 3.9. Both $\Delta L$ and $\Delta W_{\text {gate }}$ depend on mask fabrication techniques, photolithographic process and equipment, production quality control, source/drain junction depths, and the size of the minimum dimensions. In most processes $L_{e f f}$ is less than $L_{m}$ because lateral diffusion of the $\mathrm{p}^{+}$implant is dominant compared to other photolithographic variations. In the Al gate process $W_{\text {eff }}$ is always larger than $W_{\text {gate }}$ because over-etching of either or both source/drain diffusion and gate oxide is used to compensate for the misalignment in order to improve yield [29].

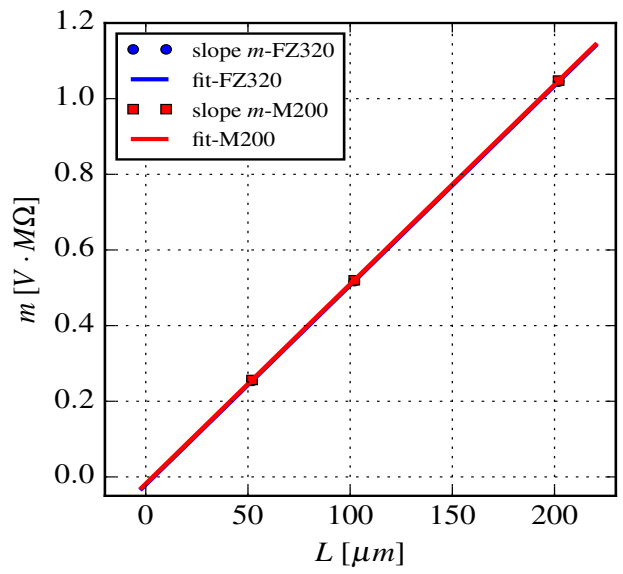

(a)

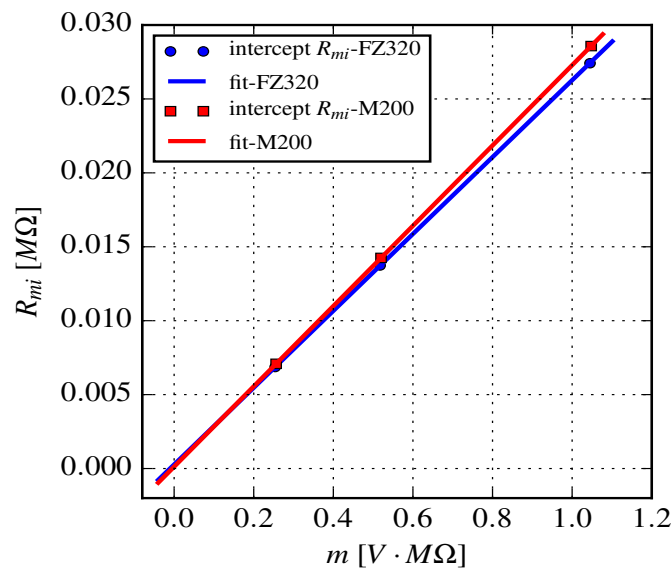

(b)

Figure 3.8: (a) Slope $m$ as a function of $L$ and (b) Intercept $R_{m i}$ as a function of $m$ for the FZ320 and M200 rectangular p-MOSFETs $<100>$. 


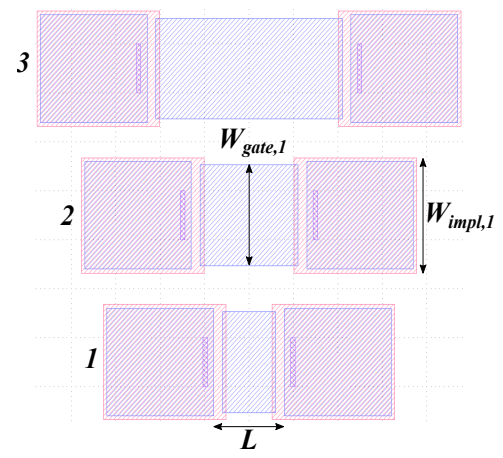

(a)

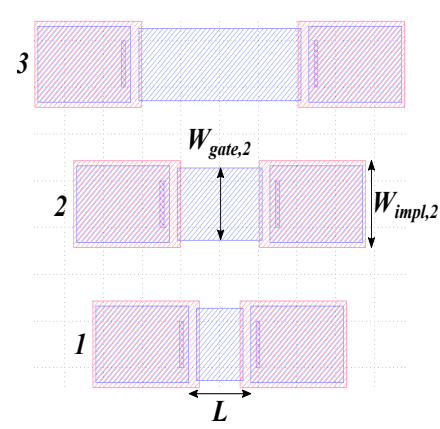

(b)

Figure 3.9: Proposed future design of rectangular MOSFETs for two different gate widths (a) $W_{\text {gate }, 1}$ and (b) $W_{\text {gate }, 2}$.

\subsection{Summary}

In this chapter are presented measurements of the drain-source current $I_{d s}$ as a function of $V_{\text {gate }}$ for long channel p-MOSFETs built on $<100>$ n-type Si. The data can be described for gate voltages approximately $3 \mathrm{~V}$ above the threshold to an accuracy of about $1 \%-2 \%$ by the standard Brews formula in the linear region and a model for the electric field dependence of the surface mobility. This allows to determine the threshold voltage to an accuracy of about $10 \mathrm{mV}$ and the surface mobility of the charge carriers in the inversion layer to an accuracy of $1 \%$. 


\section{Basic mechanisms of radiation effects and the Si- $\mathrm{SiO}_{2}$ system}

When a segmented silicon sensor is exposed to a radiation environment there will be an alteration of the electrical properties which results in degradation of sensor performance or sensor failure. The main goals of the sensor design community is understanding of the physical phenomena involved in the specific environment and define process and design rules to achieve hardness assurance. In this chapter are presented the basic mechanisms of radiation effects on materials $\mathrm{Si}, \mathrm{SiO}_{2}$ and the $\mathrm{Si}_{-} \mathrm{SiO}_{2}$ system [64].

\subsection{Fundamentals of radiation environments and sources}

\subsubsection{Radiation sources}

In general electronic systems are exposed in two main categories of radiation environments. These are space radiation and radiation from nuclear interactions and explosions as the Particle Physics or Photon Science experiments. Silicon sensors which exposed in the second category should be optimized to withstand radiation from short pulses and high dose rates. In order to study the radiation effects, facilities such as ${ }^{60} \mathrm{Co}$ cells, particle accelerators, flash X-ray machines, Synchrotron sources and nuclear reactors provide the test radiation environment.

\subsubsection{Interaction of radiation with matter}

The interaction of radiation with matter depends mainly on the mass, type, charge, and kinetic energy of the incident particle, and on the atomic mass, charge (atomic number), and density of the target material. There are a number of specific types of interaction that can occur between primary particles and target atoms, Table 4.1. In the next paragraphs are presented general features, available in Refs. [64-76].

Photon interactions Photons interact with target atoms through the photoelectric effect, Compton scattering and pair production. The energy range in which photoelectric collisions dominate depends on the atomic number, Z, of the material. The probability of a photoelectric interaction decreases with increasing photon energy and increases with Z. In Compton scattering the photon energy is much greater than the binding energy of atomic electrons, so this process does not involve complete absorption of the incident photon. The 


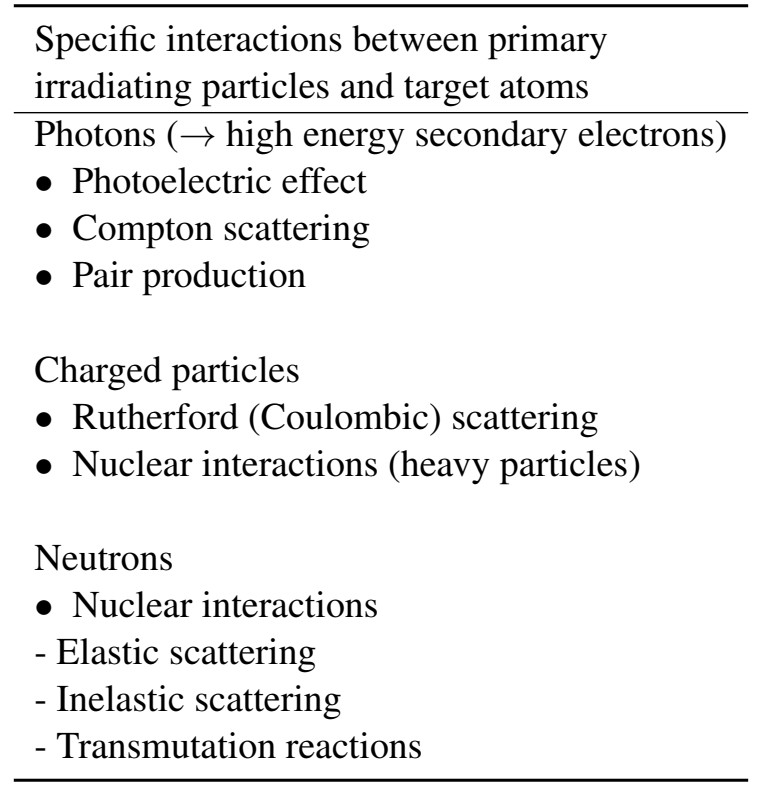

Table 4.1: Specific types of interaction between the primary incident particles and target atoms [64].

incident photon gives up a portion of its energy to scatter an atomic electron. Under this process a Compton electron is created and the lower energy scattered photon continues to travel in the target material. As the photon energy increases, Compton scattering dominates over the photoelectric effect. The third type of photon interaction is pair production and has a threshold energy of $1.02 \mathrm{MeV}$. Above this energy, a photon striking a high $\mathrm{Z}$ target material may be completely absorbed and cause a positron/electron pair to form. In Fig. 4.1 is presented the relative importance of the three photon interactions as a function of $\mathrm{Z}$ and photon energy.

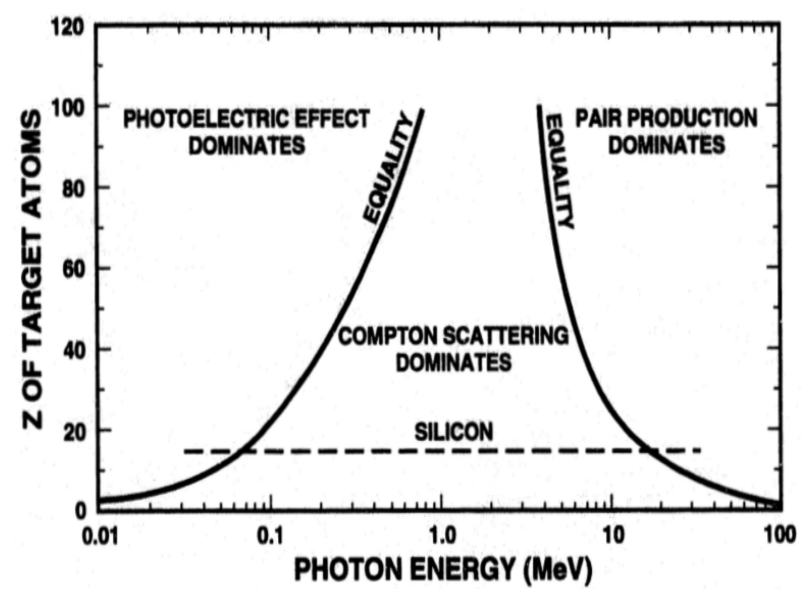

Figure 4.1: Relative importance of the three photon interactions as a function of atomic number and photon energy. Figure taken from [71].

The solid lines correspond to equal interaction cross sections for the neighboring effects. For silicon $(\mathrm{Z}=$ 14), the photoelectric effect dominates at energies below $50 \mathrm{keV}$ and pair production dominates at energies above $20 \mathrm{MeV}$. Compton scattering dominates in the energy range between these two processes. In all three cases primary energy transfer from the incident photons to the target material occurs via the secondary electrons and positrons (at very high photon energy). 
Charged particle interactions Charged particles incident on a target interact primarly by Rutherford scattering (Coulomb scattering). This interaction can cause both excitation and ionization of atomic electrons. In addition sufficient energy can be transferred to atoms to displace them from their lattice positions. Heavy charged particles can also undergo nuclear interactions if the scattering is inelastic, for example a proton can be absorbed in a target nucleus, and then the nucleus emits an alpha particle. Ionization of the target material is a major consequence of the charged particle interactions. In semiconductors as $\mathrm{Si}$ and insulators as $\mathrm{SiO}_{2}$ ionization results in production of non-equilibrium densities of electrons and holes. In general ionization related with the passage of single energetic particle through a solid, is a complex process where high energy secondary electrons are generated with various energies and momenta and produce further ionization. However, the final ionization events and the main energy transfer occur through a single type of intermediate process involving the collective motions of many valence electrons in simple oscillatory motion against the background of positive ionic cores. These plasma vibrations, or plasmons as they are called in quantum mechanics, are induced by the long range of the Coulomb interaction, which extends over regions containing many atoms. The plasmon energies, corresponding to the resonance frequency of the oscillations, are typically in the range from 10 to $20 \mathrm{eV}$ for most solids, depending upon the number density of valence electrons. A plasmon decays rapidly ( $\leq 1 \mathrm{ps}$ ) via excitation of a single electron/hole pair across the bandgap. The excess kinetic energy carried by the individual electrons and holes may result in one or two further ionization events (depending upon the bandgap width) with the remainder of the energy being quickly dissipated as thermal lattice motion.

Neutron interactions When neutrons incident a solid target the following nuclear interactions occur: elastic scattering, inelastic scattering and transmutation. For an elastic collision the neutron gives up a portion of its energy to an atom of the target material, and if this energy is higher than that required for displacement ( $\sim 25 \mathrm{eV}$ for most materials) the atom will be dislodged from its lattice position. The displaced atom is called primary recoil and can lose energy due to ionization and displace other lattice atoms. Inelastic neutron scattering involves capture of the incident neutron by the nucleus of the target atom and subsequent emission of the neutron at a lower energy. Kinetic energy is lost in this process and the target nucleus is left in an excited state. The excited nucleus returns to its original state by emission of a gamma ray. Inelastic neutron scattering can also cause displacement of the target atom to occur. The transmutation reaction involves capture of the incident neutron by the target nucleus and subsequent emission of another particle, such as proton or an alpha particle. The remaining atom is thereby transmuted, for example converted from one element into another.

Ionization and atomic displacements As mentioned above the two basic effects when a particle incidents solid state electronics are, ionization (generation of electron/hole pairs) and displacement damage (dislodging atoms from their normal lattice positions). Depending on the type of the incident particle, ionization and displacement damage can be primary or secondary effect, Fig. 4.2. In particular as shown in Fig. 4.3, ionization process in semiconductors and insulators occurs when an electron in the valence band is excited across the bandgap into a conduction band state, either as a direct result of interaction with an energetic charged particle or as the result of the decay of a plasmon excitation [77]. In the order of picosecond the excited electron in the conduction band and the hole left behind in the valence band lose their excess kinetic 
energy through lattice scattering and are "thermalized" in energy, falling to the vicinity of the conduction and valence band edges, respectively.

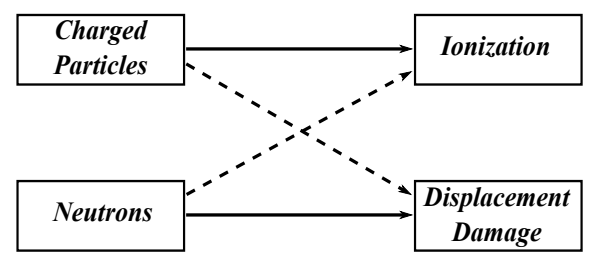

Figure 4.2: Schematic indicating primary radiation effects (solid lines) and secondary effects (dashed lines) in electronic materials [64].

Except for some fraction (small in semiconductors, possibly large in insulators) of the electron/hole pairs which undergo what is called initial recombination, the electron and hole will be free to diffuse and drift (if electric fields are present) away from their point of generation. Until they either undergo recombination elsewhere in the material, are trapped at a localized trap site, or are collected at an electrode.
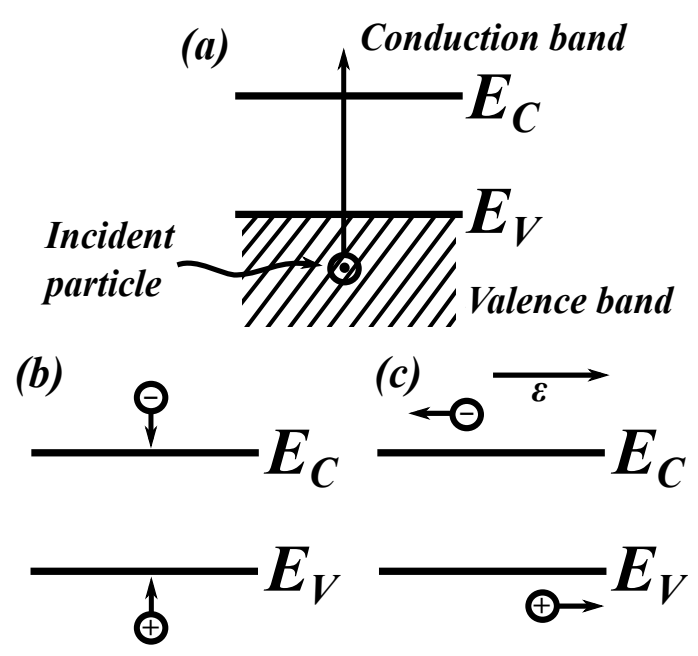

Figure 4.3: Process of ionization in semiconductors and insulators: (a) Ionization event, (b) Thermalization (picosecond time scale), and (c) Charge separation and drift. Ionization leads to transient photocurrents and build-up of trapped charge (space-charge effects). Effective measure of damage is charge yield per unit dose.

Figure adapted from [64].

If an electric field is present, there will be a net charge separation and therefore, an electric current. These radiation-induced photocurrents can be a major problem in semiconductor junction regions, resulting in transient upsets of circuits or current latch-up conditions. These problems are increasing in importance as the sizes of devices are scaled down, so less charge is necessary to cause failure. In insulators (e.g $\left.\mathrm{SiO}_{2}\right)$, radiation-induced photocurrents are generally not a problem because of the much lower carrier mobilities and lower numbers of electron/hole pairs created. The insulators generally contain relatively large densities of charge trapping centers at which the radiation-induced charges can be trapped for long periods of time. The trapped charges can then generate internal space-charge electric fields, which in turn can lead to voltage offsets or shifts in device operating characteristics. In addition, internal space charge fields due to trapped 
charge in field oxides and passivation insulators can turn on parasitic current leakage paths. The amount of damage due to ionization is directly related to the charge yield per unit dose, i.e., number of electron/hole pairs generated per Gy. Table 4.2 presents for $\mathrm{Si}$ and $\mathrm{SiO}_{2}$ the average ionization energy $\left(\mathrm{E}_{P}\right)$ required to generate a single electron/hole pair, as well as the initial charge pair density per Gy $\left(\mathrm{g}_{0}\right)$ deposited in the material.

\begin{tabular}{lll}
\hline Material & $\begin{array}{l}\text { Pair generation energy, } \\
\mathrm{E}_{P}[\mathrm{eV}]\end{array}$ & $\begin{array}{l}\text { Pair density generated per } \\
\mathrm{Gy}, \mathrm{g}_{0}\left[\mathrm{~cm}^{-3} \mathrm{~Gy}^{-1}\right]\end{array}$ \\
\hline Silicon & 3.6 & $4.0 \times 10^{15}$ \\
Silicon dioxide & 17 & $8.1 \times 10^{14}$ \\
\hline
\end{tabular}

Table 4.2: Electron-hole pair generation energies and pair densities generated by $1 \mathrm{~Gy}$.

The latter quantity is obtained from the product of the material density and the deposited energy per Gy $\left(6.24 \times 10^{15} \mathrm{eV} / \mathrm{g}\right)$ divided by $\mathrm{E}_{P}$. In wide bandgap insulators such as $\mathrm{SiO}_{2}$, there can be significant initial (or immediate) recombination of the electron/hole pairs before they can separate. The actual charge yield in this case is a function of the electric field and the line density of electron/hole pairs (number created per track length of the incident particle). The value of $\mathrm{g}_{0}$ listed in Table 4.2 corresponds to the yield in the high field limit. The important outcome of the atomic displacements is that defects are produced in the crystal lattice. These may be simple defects such as vacancies and interstitials, or simple combinations of these such as divacancies, or complexes of vacancies and interstitials with impurity atoms, or even more complex clusters or defects. Fig. 4.4 shows a simple vacancy defect at the initial lattice site and a simple interstitial where the knock-on atom came to rest. Defects or impurities which disturb the lattice periodicity produce localized, discrete energy levels lying within the forbidden bandgap of the perfect lattice [78]. These electronic energy levels cause an alteration of the electrical properties of the semiconductor crystal and lead to degradation of the device. For example, additional recombination centers are introduced which can shorten the minority carrier lifetimes.

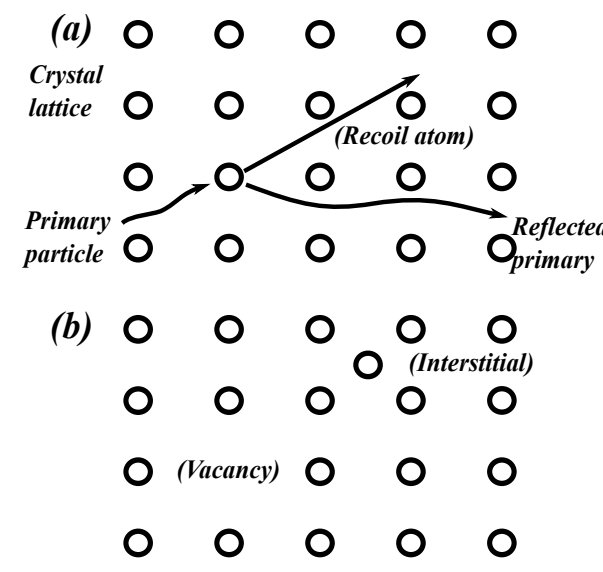

Figure 4.4: Atomic displacement damage in crystalline solid: (a) Atomic displacement event and (b) Simple radiation-induced defects (vacancy and interstitial). Atom displacements produce lattice defects which result in localized trap states-energy levels within bandgap. Figure adapted from [64].

Terminology of radiation exposure Neutron exposure is commonly given in terms of neutron fluence, $\mathrm{n} / \mathrm{cm}^{2}$. The amount of displacement damage in a certain material varies significantly with neutron energy. In 
order to allow meaningful comparisons between experiments using different neutron energy spectra, neutron fluences are expressed in terms of ("normalized to") an equivalent radiation damage of $1 \mathrm{MeV}$ neutron fluence. For charged particle exposure, the amount of energy that is lost into ionization is given by the stopping power, or the linear energy transfer (LET) function $\rho^{-1} d E / d x$, expressed in units of $\mathrm{MeV} \cdot \mathrm{cm}^{2} / \mathrm{g}$. The stopping power has been studied for a number of target materials as a function of incident particle energy and atomic number. An example of the stopping power for electrons and protons incident on silicon is presented in Fig. 4.5. The absorbed ionizing dose $(D)$ is the integral over energy of the product of the particle energy spectrum and the stopping power. The commonly used unit in the space radiation effects community of absorbed ionizing dose is the rad (radiation absorbed dose). One rad is equal to an absorbed energy of 100 ergs per gram of material. The energy loss per unit mass differs from one material to another, the material in which the dose is deposited must be specified, e.g dose $(\mathrm{Si})$ or $\operatorname{dose}\left(\mathrm{SiO}_{2}\right)$. In the further chapters, the SI unit Gray (Gy) will be used for the calculation of the absorbed dose, where $1 \mathrm{~Gy}$ is equal to an absorbed energy of $1 \mathrm{~J} / \mathrm{kg}$ or $100 \mathrm{rad}$. The ionizing dose rate $(\dot{D})$ is expressed in Gy/s [79].

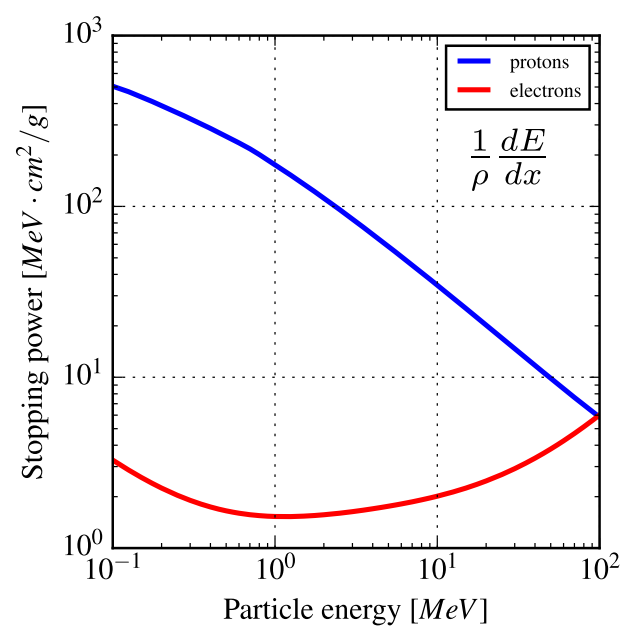

Figure 4.5: Stopping power as a function of particle energy for electrons and protons incident on silicon. Data taken from [79].

\subsection{Properties of $\mathrm{SiO}_{2}$ and the $\mathrm{Si}-\mathrm{SiO}_{2}$ interface}

\subsubsection{Silicon dioxide $\mathrm{SiO}_{2}$}

Silicon dioxide, $\mathrm{SiO}_{2}$, is probably the most important material in silicon technology and device processing. It can be used as dielectric capacitor and isolation material, in the particular device where it is formed. Also it is used many times during silicon processing as a masking material for diffusion or etching and cleaning of the silicon surface.

\subsubsection{Oxidation process}

Silicon itself is an easily oxidized material. Depending on the surface conditions a native oxide of nanometer thickness grows on the silicon surface. This oxide is limited in thickness and it is not pure stoichiometric 
$\mathrm{SiO}_{2}$. Chemical Vapor Deposited CVD oxides are used in many applications however superior silicon dioxides are grown at 800 to $1200{ }^{\circ} \mathrm{C}$, Fig. 4.6. Two basic schemes are used: wet and dry oxidation

Wet oxidation: $\mathrm{Si}(\mathrm{s})+2 \mathrm{H}_{2} \mathrm{O}(\mathrm{g}) \longrightarrow \mathrm{SiO}_{2}(\mathrm{~s})+2 \mathrm{H}_{2}(\mathrm{~g})$

Dry oxidation: $\mathrm{Si}(\mathrm{s})+\mathrm{O}_{2}(\mathrm{~g}) \longrightarrow \mathrm{SiO}_{2}(\mathrm{~s})$

In general thermal oxidation is a slow process, however wet is faster than dry oxidation. In particular, the growing rate of the oxide on the silicon surface depends on the silicon crystal orientation, the doping concentration of the material and the oxygen pressure in the furnace. Usually thin oxides in the order up to $20 \mathrm{~nm}$ are grown in dry oxidation and thicker in wet oxidation.

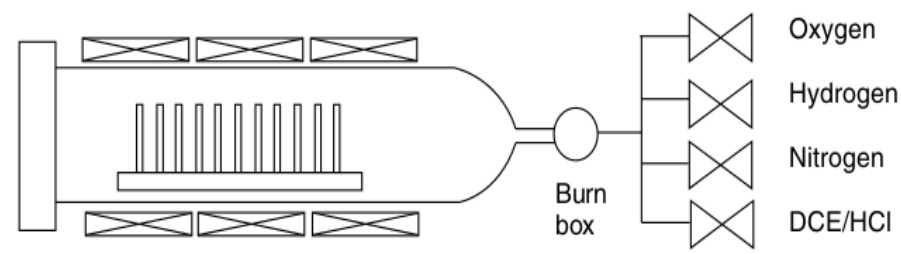

Figure 4.6: Horizontal oxidation furnace with 3-zone resistive heating: wafers are vertically loaded in quartz boats. Figure taken from [80].

\subsubsection{Oxide structure}

The thermally grown silicon dioxide is glassy with basic unit the silica structure $\mathrm{SiO}_{4}$, Fig. 4.7 (a). It presents only short-range order with un-bonded oxygen charged atoms and it is less stable than quartz crystalline $\mathrm{SiO}_{2}$. Silicon dioxide has density $2.3 \mathrm{~g} / \mathrm{cm}^{3}$ and Young modulus $87 \mathrm{GPa}$. In order to grow oxide layer of thickness $t$, a silicon thickness $0.45 t$ has to be consumed. Under this volume change the original silicon surface is below the oxide mid-point and mechanical stresses appear on the surface and in corners of structures. In addition the volume change causes vacancies at the interface between silicon and oxide and many atoms have free dangling bonds, Fig. 4.7 (b).

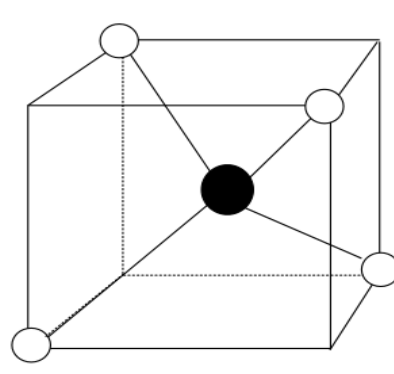

(a)

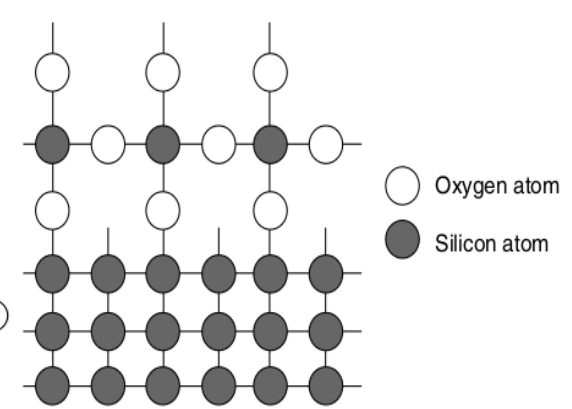

(b)

Figure 4.7: (a) Basic structure of silica: a silicon atom tetrahedrally bonds to four oxygen atoms, (b) The structure of silicon-silicon dioxide interface: some silicon atoms have dangling bonds. Figures taken from [80].

These bonds act as interface trap centers of charge carriers. In order to anneal out defects after thermal oxidation post-oxidation anneal in nitrogen and hydrogen environment is used. In particular the hydrogen 
passivates the free dangling bonds and reduces the charge trapping. For the characterization of the silicon oxide quality electrical measurements such as the breakdown voltage tests are performed. High quality silicon dioxides can sustain up to $12 \mathrm{MV} / \mathrm{cm}$ electric fields, however typical silicon oxides have breakdown fields up to $5 \mathrm{MV} / \mathrm{cm}$. The $\mathrm{E}^{\prime}$ center, usually observed in irradiated MOS devices, consist of two Si atoms joined by a week, strained $\mathrm{Si}-\mathrm{Si}$ bond with a missing oxygen atom, sometimes referred to as an oxide vacancy, shown in Fig. 4.8. It is one of the most dominant radiation-induced defects. E' centers also preexist in oxide films due to the amorphous nature of $\mathrm{SiO}_{2}$ and thermodynamic considerations. Each $\mathrm{Si}$ atom is back bonded to three oxygen atoms. When a positive charge is captured, the Si-Si bond breaks [81].

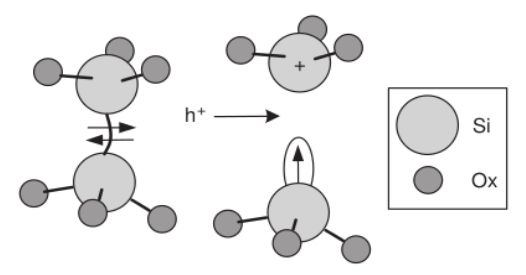

Figure 4.8: Model for hole trapping and $\mathrm{E}^{\prime}$ center formation in $\mathrm{SiO}_{2}$. Figure taken from [81].

The structure of neutral electron traps can be explained by $\mathrm{E}^{\prime}$ centers. It is established that the $\mathrm{E}^{\prime}$ center is formed by breaking the Si-Si bond in an oxygen vacancy defect, Fig. 4.9 (a). The bond breaking is facilitated by the capture of a hole, Fig. 4.9 (b), leaving a positively charged trap and one Si atom with a dangling orbital containing one unpaired electron. The resonant flipping of the spin of this unpaired electron gives rise to the $\mathrm{E}^{\prime}$ signal in Electron Spin Resonance [82].

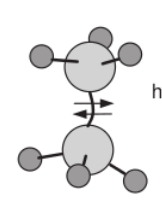

(a)

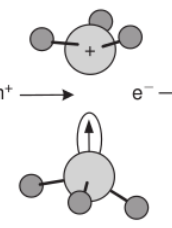

(b)

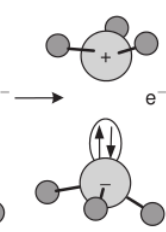

(c)

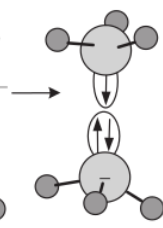

(d)

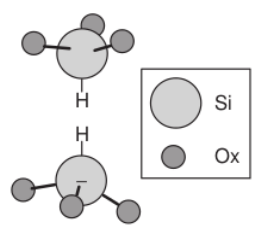

(e)

Figure 4.9: Nature of neutral electron traps. Figure taken from [81].

Upon electron capture, the center can return to the $\mathrm{E}^{\prime}$ center or the electron from one of the $\mathrm{Si}$ atoms decays to a ground state by joining the unpaired electron of the other Si atom forming a neutral amphoteric trap, Fig. 4.9 (c). Capture of a second electron leaves it negatively charged, Fig. 4.9 (d). This electron trapping event gives rise to the threshold voltage shifts associated with filled neutral electron traps. Attempts to anneal neutral traps have been only partially successful. The hydrogen anneal model is shown in Fig. 4.9 (e).

\subsection{4. $\mathrm{Si}^{-\mathrm{SiO}} \mathrm{O}_{2}$ interface}

Interface traps exist at the $\mathrm{Si}_{-} \mathrm{SiO}_{2}$ interface and they are the result of a structural imperfection. Silicon is tetrahedrally bonded with each $\mathrm{Si}$ atom bonded to four $\mathrm{Si}$ atoms in the wafer bulk. When the Si is oxidized, the bonding configuration at the surface is as shown in Figs. 4.9 (a) and 4.9 (b) with most Si atoms bonded to oxygen at the surface. Some Si atoms bond to hydrogen, but some remain unbonded. An interface trap, is an interface trivalent $\mathrm{Si}$ atom with an unpaired valence electron usually denoted by $\mathrm{Si}_{3} \equiv \mathrm{Si} \bullet$, where the $(\equiv)$ represents three complete bonds to other Si atoms (the $S i_{3}$ ) and the $(\bullet)$ represents the fourth, unpaired 
electron in a dangling bond. Interface traps, are known as $P_{b}$ centers [83, 84]. On $<111>$ oriented $\mathrm{Si}$, the $P_{b}$ center is situated at the $\mathrm{Si}-\mathrm{SiO}_{2}$ interface with its unbonded central atom orbital perpendicular to the interface and aimed into a vacancy in the oxide as shown in Fig. 4.10 (a). It has been detected by ESR as shown in Fig. 4.11.

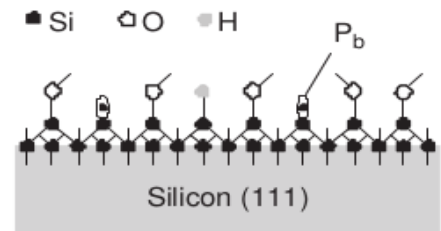

(a)

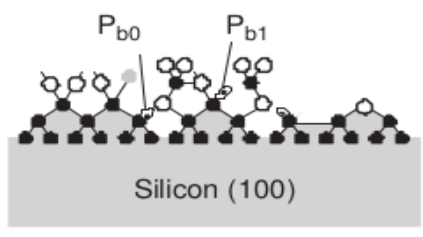

(b)

Figure 4.10: Structural model of the (a): $<111>$ Si surface and (b): $<100>$ Si surface. Figure taken from [81].

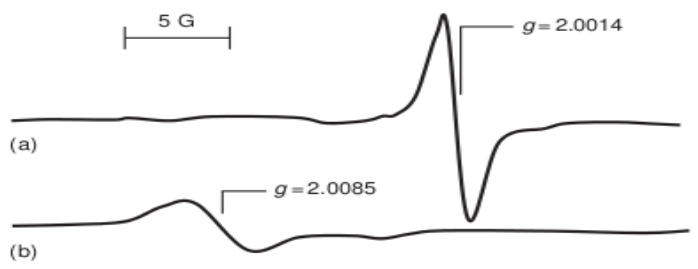

Figure 4.11: ESR traces of the $P_{b}$ center for the magnetic field (a) parallel and (b) perpendicular to the $<111>$ symmetry axis. Figure taken from [81].

On $<100>$ oriented $\mathrm{Si}$, the four tetrahedral $\mathrm{Si}-\mathrm{Si}$ directions intersect the interface plane at the same angle. Two defects, named $P_{b 1}$ and $P_{b 0}$ are shown in Fig. 4.10 (b) and they have been detected by ESR. It is known that both $P_{b 0}$ and $P_{b 1}$ are chemically identical to the $P_{b}$ center. Interface traps are electrically active defects with an energy distribution $D_{i t}\left[1 /\left(\mathrm{eV} \cdot \mathrm{cm}^{2}\right)\right]$ throughout the Si bandgap, Fig 4.12.

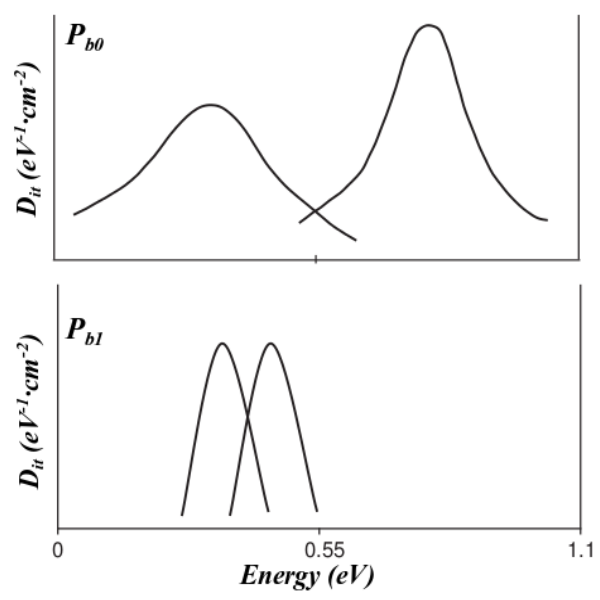

Figure 4.12: A schematic illustration of the $P_{b 0}$ and $P_{b 1}$ densities of states. Figure taken from [81].

The surface potential dependence of the occupancy of interface traps is shown in Fig. 4.13. Interface traps at the $\mathrm{Si}-\mathrm{SiO}_{2}$ interface are acceptor-like in the upper half and donor-like in the lower half of the Si bandgap [85]. So far, as shown in Fig. 4.13 (a), at flatband, electrons occupying states below the Fermi level $E_{F}$, the states in the lower half of the bandgap are neutral (occupied donors labeled by "0"). Those between midgap and $E_{F}$ are negatively charged (occupied acceptors labeled by "-"), and those above $E_{F}$ are neutral (unoccupied acceptors). For a p-MOSFET in inversion, shown in Fig. 4.13 (b), the fraction of 
interface traps between the midgap and $E_{F}$ is now unoccupied donors, leading to positively charge interface traps (labeled by "+").

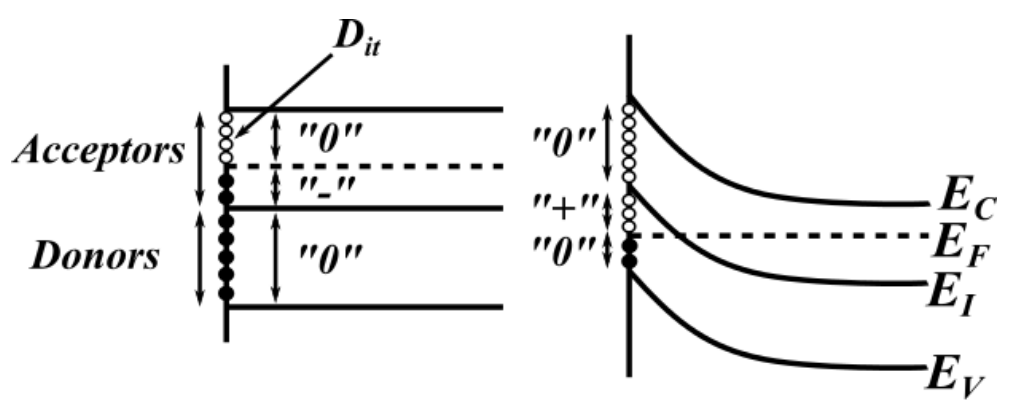

(a)

(b)

Figure 4.13: Band diagrams of p-MOSFET (n-type Si) showing the occupancy of interface traps with (a) negative interface trap charge at flatband and (b) positive interface trap charge at inversion. Figure adapted from [81].

\subsubsection{Nomenclature of oxide charges}

Some impurities or defects can be inadvertently incorporated into the oxide during oxide growth or subsequent processing steps. This results in the oxide being contaminated with various types of charges and traps. Four different types of charges have been identified in thermally grown oxide on a silicon surface. These charges are shown schematically in Fig. 4.14. They are 1) Mobile ionic charge $Q_{m}, 2$ ) Fixed oxide charge $\left.Q_{f}, 3\right)$ Oxide trapped charge $Q_{o t}$ and 4) Interface trapped charge $Q_{i t}$, according to Refs. [4,29]. Later Dan Fleetwood suggested to add the term border traps as shown in Fig. 4.14. The densities of these charges are very much dependent on the device fabrication process.

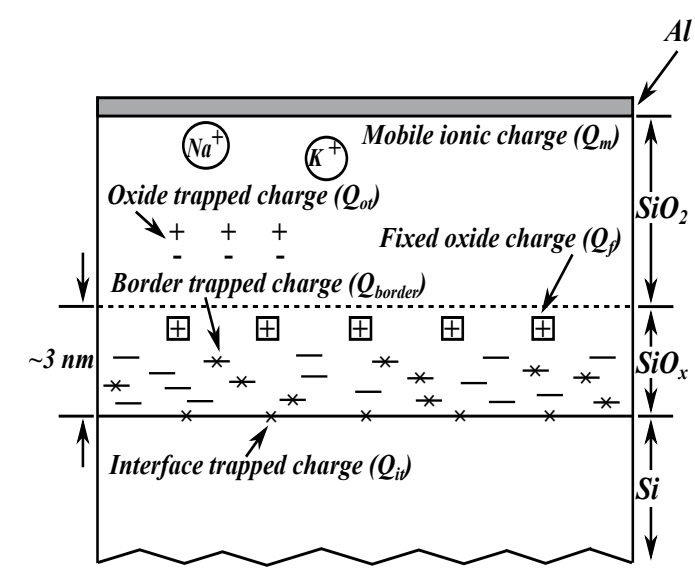

Figure 4.14: Nomenclature of oxide charges $[86,87]$.

Mobile oxide charge $\left(Q_{m}, N_{m}\right)$ : The mobile ionic charges $Q_{m}$ are due to sodium $\left(\mathrm{Na}^{+}\right)$or other alkali ions that gets into the oxide during cleaning, processing and handling of the MOS devices. These ions move very slowly within the oxide. Their transport depends strongly on the applied electric field $(\sim 1 \mathrm{MV} / \mathrm{cm})$ 
and temperature $\left(30-400^{\circ} \mathrm{C}\right)$. Positive voltages push the ions towards the interface, while negative voltages draw them towards the gate. Device instabilities from mobile ions are minimized by avoiding contamination during processing.

Fixed oxide charge $\left(Q_{f}, N_{f}\right)$ : As the name suggests these are the immobile charges located within approximately $25 \AA$ of the $\mathrm{Si}_{-} \mathrm{SiO}_{2}$ interface and normally arise from structural damage associated with oxidation or various impurity atoms. Generally $Q_{f}$ is positive and depends on the oxidation ambient, temperature and annealing conditions and silicon orientation. It is independent on the doping type and concentration in the silicon, oxide thickness and oxidation time.

Oxide trapped charge $\left(Q_{o t}, N_{o t}\right)$ : The oxide trapped charge $Q_{o t}$ is associated with defects in $\mathrm{SiO}_{2}$. The oxide traps are usually electrically neutral and are charged by introducing electrons and holes into the oxide through ionizing radiation. The magnitude of $Q_{o t}$ depends on the amount of radiation dose and energy and the field across the oxide during irradiation. $Q_{o t}$ resembles $Q_{f}$ in that its magnitude is not a fraction of silicon surface potential and there is no capacitance associated with it.

Interface trapped charge $\left(Q_{i t}, N_{i t}\right)$ : The interface trapped charge, is the charge due to electronic energy levels located at the $\mathrm{Si}_{-} \mathrm{SiO}_{2}$ interface with energy states in the silicon bandgap that can capture or emit electrons or holes. These electronic states arise because of the lattice mismatch at the interface, dangling bonds, the adsorption of foreign impurity atoms at the silicon surface and other defects caused by radiation or similar bond breaking processes. These are the most important type of charges because of their wide-ranging and degrading effect on device behavior. Under equilibrium condition, the occupancy of these interface states or traps is governed by the position of the Fermi level. The interface trap levels are distributed across the silicon energy band, and the interface trap density $D_{i t}$, is defined

$$
D_{i t}=\frac{1}{q_{0}} \frac{d Q_{i t}}{d E} \quad \text { number of charges } / \mathrm{eV} \cdot \mathrm{cm}^{2}
$$

is extremely sensitive to even minor process details, varies significantly from process to process and is orientation dependent.

Border traps $\left(Q_{b t}, N_{b t}\right)$ : These traps have been introduced by Dan Fleetwood [87] as slow states, near interface oxide traps, switching oxide traps and by other names. The border traps are those near interfacial oxide traps located within approximately $3 \mathrm{~nm}$ of $\mathrm{Si}_{-} \mathrm{SiO}_{2}$ interface. There is no distinct depth limit, however, border traps are considered to be those traps that can communicate with the silicon through capture and emission of electrons and holes on the time scale of interest [4].

In the MOS defect literature, there are wide varieties of nomenclatures used to characterize defects in materials, devices and circuits. The different terms that are often used to describe defects that are similar or even identical in microstructure can vary with the method of characterization, the effect of the defect on the device of interest, the background of the investigator, the convention of the particular technical community 
and many other factors. In Fig. 4.15, a representative sampling is presented of many present and historical terms that are used to describe defects in the $\mathrm{Si}_{-} \mathrm{SiO}_{2}$ system. The defects are grouped schematically with their physical location in the $\mathrm{Si}$ bulk, at the $\mathrm{Si}-\mathrm{SiO}_{2}$ interface, in the near-interfacial $\mathrm{SiO}_{2}$, in the $\mathrm{SiO}_{2}$ bulk and at the gate/ $/ \mathrm{SiO}_{2}$ interface [81].

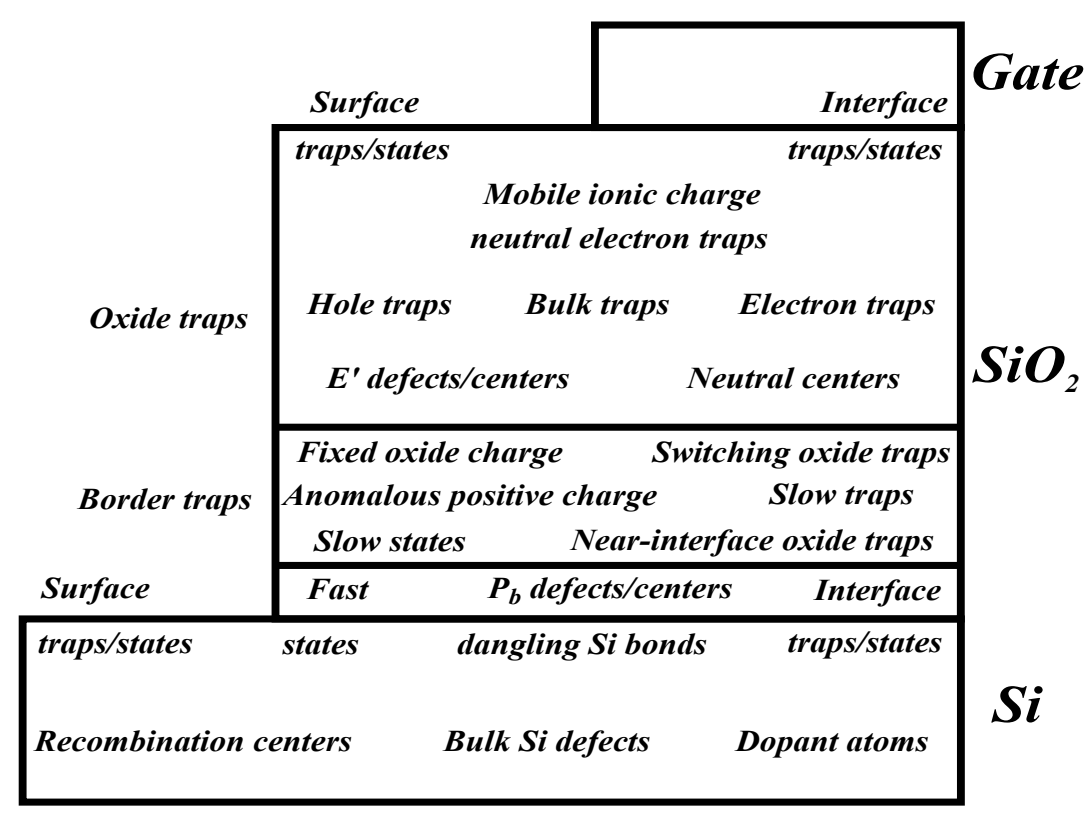

Figure 4.15: Schematic diagram that associates common names applied to defects in MOS devices with $\mathrm{SiO}_{2}$ gate dielectrics with their approximate spatial locations. Figure adapted from [81].

\subsection{Mechanisms of the build-up of radiation-induced surface damage}

The part of an MOS structure most sensitive to ionizing radiation is the oxide insulating layer $\left(\mathrm{SiO}_{2}\right)$, which in present-day silicon sensors is a few hundred nanometers. When the radiation passes through the oxide, the energy deposited creates electron/hole pairs. In $\mathrm{SiO}_{2}$, the radiation generated electrons are much more mobile than the holes, and they are swept out of the oxide (collected at the gate electrode) in times on the order of picoseconds. In the first picosecond or two, a fraction of electrons and holes recombine. This fraction depends on the applied field and on the energy and type of the incident particle [64]. The holes which escape initial recombination are relatively immobile and remain near their points of generation, causing negative voltage shifts in the electrical characteristics of MOS devices. Over a period of time extending typically at room temperature from $10^{-7} \mathrm{~s}$ to the order of seconds the holes undergo a rather anomalous stochastic hopping transport through the oxide in response to any electric fields present. When the holes reach the $\mathrm{SiO}_{2}$ interface (for positive applied gate bias), some fraction of them are captured in long-term trapping sites and cause a negative voltage shift. This long-lived radiation-induced voltage shift component is the most commonly observed form of radiation damage in MOS devices. The long-term trapping of holes near the $\mathrm{Si}_{-} \mathrm{SiO}_{2}$ interface, as well as their subsequent annealing in time, constitutes the third major factor of 
MOS response as presented in Fig. 4.16, hole trapping and annealing are very sensitive to the processing of the oxide and to other variables such as field and temperature.

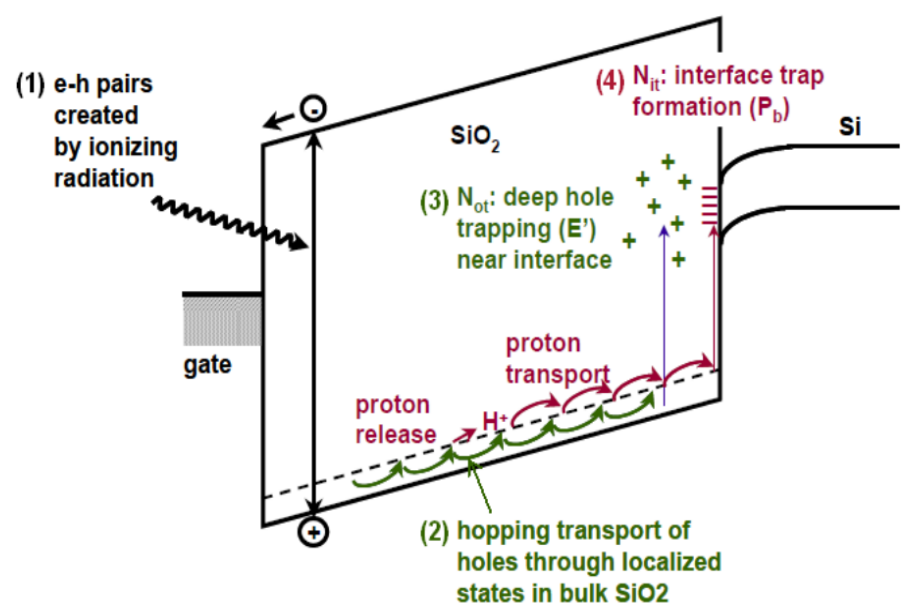

Figure 4.16: Schematic energy band diagram of $\mathrm{SiO}_{2}$, MOS structure for positive gate bias, indicating major physical processes underlying radiation exposure. Figure taken from $[75,88]$.

The fourth and final component of MOS response is that of a radiation-induced build-up of interface traps. There can be both prompt interface traps, present immediately after irradiation, as well as a delayed timedependent build-up of states which can continue for hours at room temperature. Both the magnitude and nature (relative ratio of prompt and delayed components) of the interface traps are also highly dependent upon oxide processing, as well as upon other variables such as temperature and applied field (both magnitude and polarity). A major electrical consequence of the radiation-induced charging of the $\mathrm{SiO}_{2}$ film (including transporting holes, trapped holes and interface traps) is a shift in voltage operating points for devices, such as the threshold voltage $V_{t h}$ of a MOSFET. It can be written as

$$
V_{t h}(t)=V_{t h}^{0}(t)+\Delta V_{t h}(t)
$$

where $V_{t h}^{0}$ is the threshold voltage before irradiation and $\Delta V_{t h}(t)$ is the voltage shift following radiation exposure. The radiation-induced threshold voltage shift can be written into three components

$$
\Delta V_{t h}(t)=\Delta V_{s t}(t)+\Delta V_{o t}(t)+\Delta V_{i t}(t)
$$

where $\Delta V_{s t}(t)$ is the short term contribution from the radiation-generated mobile holes in the oxide bulk, $\Delta V_{o t}(t)$ the deep trapped holes near the interface and $\Delta V_{i t}(t)$ is the contribution from the charged interface traps

$$
\begin{array}{r}
\Delta V_{s t}=-q_{0} / C_{o x} \int_{0}^{t_{o x}} d x\left(x / t_{o x}\right) n_{h}(x, t) \\
\Delta V_{o t}=-q_{0} \Delta N_{o t}(t) / C_{o x} \\
\Delta V_{i t}=-\Delta Q_{i t}(t) / C_{o x}
\end{array}
$$


where $q_{0}$ is the electronic charge, $t_{o x}$ is the oxide thickness and $C_{o x}$ is the oxide capacitance per unit area. In Eq. 4.4, $n_{h}(x, t)$ is the space- and time-dependent density of free (mobile) holes, and the distance $x$ in the oxide is measured relative to the gate/ $\mathrm{SiO}_{2}$ interface. In Eq. 4.5, $\Delta N_{o t}(t)$ is the radiation-induced density of deep trapped holes near the $\mathrm{Si}_{-} \mathrm{SiO}_{2}$ interface. It is time dependent both because of its time dependent build-up as the transporting holes reach the interface and because of its long-term annealing. In Eq. 4.6, the sign of the radiation-induced interface trapped charge $\Delta Q_{i t}(t)$ is left unspecified, as it can contribute either a net negative or net positive charge depending on the position of the Fermi level at the Si surface at inversion. The pre-irradiation condition $t=0^{-}$is depicted in Fig. 4.17 (a)-no oxide charges.

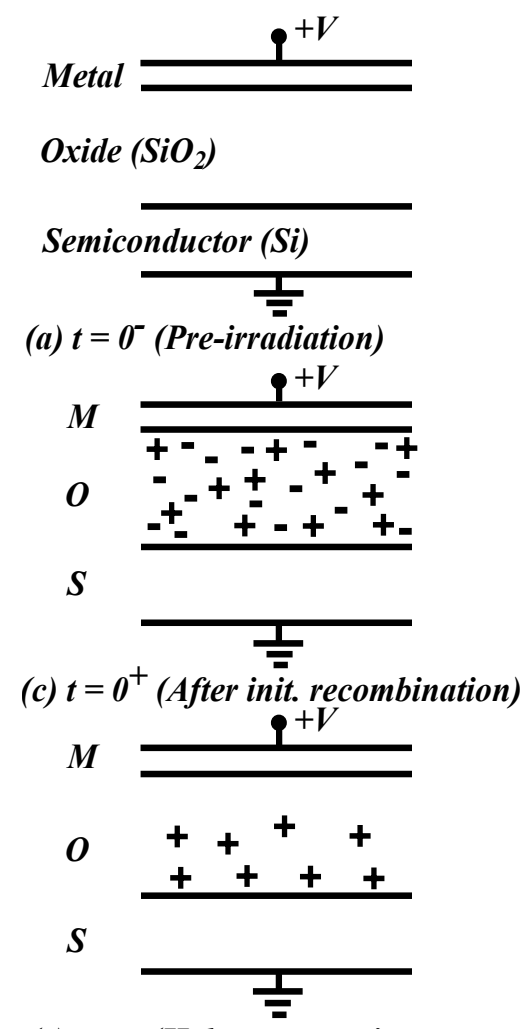

(e) $t=t_{1}$ (Hole transport in progress)

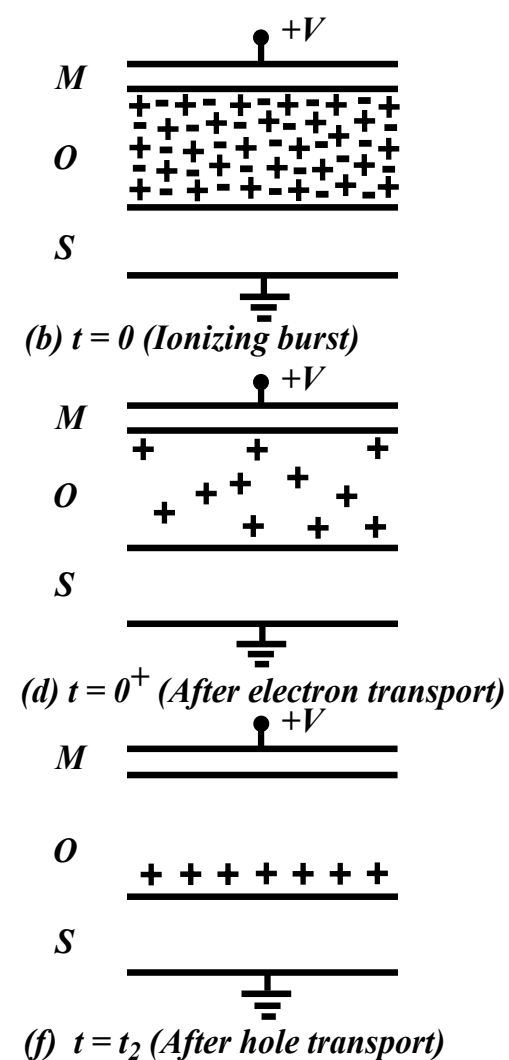

Figure 4.17: Schematic illustration of processes of charge generation and initial recombination, hole transport, and long-term trapping near the $\mathrm{Si}_{-} \mathrm{SiO}_{2}$ interface. Figure adapted from [70].

At $t=0$ Fig. 4.17 (b), the radiation pulse occurs, generating electron/hole pairs across the oxide bulk. In a time of the order of picoseconds at $t=0^{+}$Fig. 4.17 (c), some of the electron/hole pairs will recombine and the relatively highly mobile electrons will move toward the gate and be collected, Fig. 4.17 (d). Then the holes begin their relatively slow hopping move toward the $\mathrm{Si}_{-} \mathrm{SiO}_{2}$ interface, where a fraction for them are captured in the trapping sites. Fig. 4.17 (e) at $t=t_{1}$ shows the intermediate situation, where some holes are still transporting, some have been collected by the substrate electrode and some have been trapped near the interface. The final charge configuration at $t=t_{2}$ after completion of the hole transport is depicted in Fig. 4.17 (f), where only the long-term trapped holes remain near the $\mathrm{Si}_{-} \mathrm{SiO}_{2}$ interface. In general the situation is more complicated as there can be long-term annealing of the deeply trapped holes via tunneling of electrons from the Si substrate. The radiation-induced interface traps can be present both right after irradiation $t=0^{+}$as well as continuing to build-up over long time periods. The interface traps not only 
contribute to the shift of device characteristics but also cause a distortion ("stretchout") of the curves (C-V or I-V) because the charge state of the traps is dependent upon the surface potential.

\subsubsection{Charge generation and recombination in $\mathrm{SiO}_{2}$}

The initial hole yield in the oxide determines the initial (maximum) voltage shifts of MOS devices and sets the scale for the amount of damage due to ionizing radiation. The two major factors that determine the initial hole density are the electron/hole pair creation energy and the field dependent fraction of holes which escapes the initial recombination processes. The initial value of the threshold voltage shifts is then simply related to the initial hole density via the dose and geometric (oxide thickness) factors. The electron/hole pair creation energy $E_{P}$ was determined by Benedetto and Boesch which establishes $E_{P}$ to be $17 \pm 1 \mathrm{eV}$. The initial density is quickly reduced by the initial recombination processes occurring in picoseconds, before the electrons are swept out of the oxide and collected. The fraction of holes escaping initial recombination, $f_{y}\left(E_{o x}\right)$, which determines the final hole yield, is determined mainly by two factors: the magnitude of the oxide electric field $E_{o x}$, which is acting to separate the charge pairs, and the initial line density of electron/hole pairs created by the incident radiation particle. The pair line density, which is determined by the linear energy transfer (LET) and therefore a function of the incident particle type and energy, is inversely proportional to the average separation distance between electron/hole pairs. The closer the average spacing of the pairs, the more recombination that occurs for a given field, and the less will be the final yield of holes. Fig. 4.18 is a compilation of a number of experimental results of the fractional hole yield $f_{y}$ versus electric field for a number of particles spanning the range from low to high LET. For these particle sources at a field of 1 $\mathrm{MV} / \mathrm{cm}$, the yield varies from almost 90 percent for low LET particles $\left(12 \mathrm{MeV}\right.$ electrons and $\left.{ }^{60} \mathrm{Co}\right)$ to only about 6 percent for the high LET $2 \mathrm{MeV}$ alpha particles.

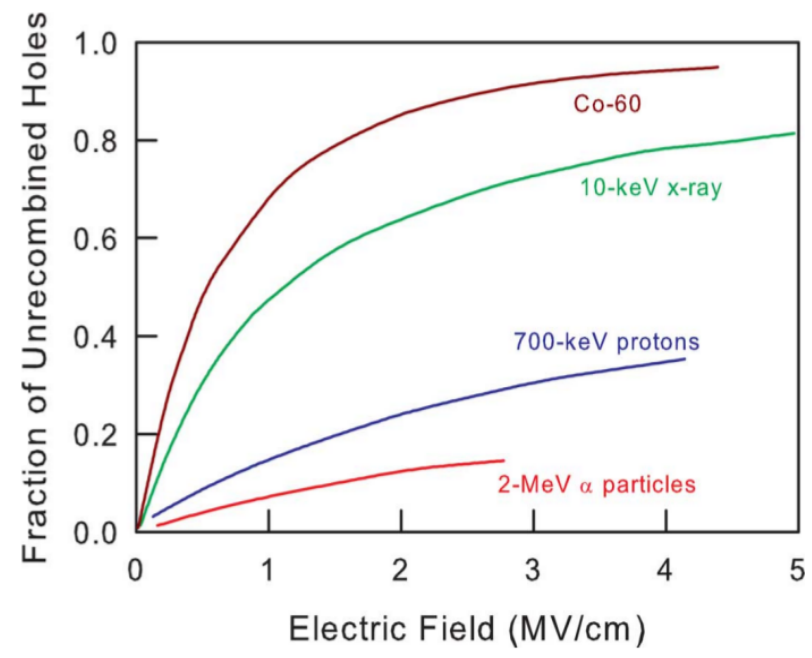

Figure 4.18: Experimentally measured fractional hole yield as a function of the electric field in $\mathrm{SiO}_{2}$ for a number of incident particles. Figure taken from [75].

Knowing the initial pair volume density per $\mathrm{Gy}\left[g_{0}=8.1 \times 10^{14} \mathrm{~cm}^{-3} \mathrm{~Gy}^{-1}\left(\mathrm{SiO}_{2}\right)\right]$ and the fractional hole yield after recombination $f_{y}\left(E_{o x}\right)$, the initial threshold voltage shift is easily obtained. Assuming a uniform generation density across the oxide layer, the total initial areal charge density of holes which escape recombination is $\Delta Q_{h}=q_{0} g_{0} t_{o x} f_{y}\left(E_{o x}\right) D$, where $t_{o x}$ is the oxide thickness and $D$ is the dose in $\mathrm{Gy}\left(\mathrm{SiO}_{2}\right)$. According to Ref. [64] the initial threshold shift is related to $\Delta Q_{h}$ as 


$$
-\Delta V_{T}\left(0^{+}\right)=\Delta Q_{h} / 2 C_{o x}=\left[q_{0} g_{0} t_{o x} f_{y}\left(E_{o x}\right) D\right] / 2 C_{o x}
$$

the factor of 2 comes from the fact that the centroid of a uniform charge density is $t_{o x} / 2$, and a negative voltage shift is explicitly indicated corresponding to the positive sign of the induced charge. Finally, substituting for the constant factors in Eq. 4.7, is derived

$$
-\Delta V_{T}\left(0^{+}\right)=1.9 \times 10^{-10} t_{o x}^{2} f_{y}\left(E_{o x}\right) D
$$

The units of $\Delta V_{T}$ are volts if $t_{o x}$ is expressed in nanometers and $D$ in $\mathrm{Gy}\left(\mathrm{SiO}_{2}\right)$. Eq. 4.8 shows the dependence of the initial voltage shifts on oxide thickness squared, indicating a significant improvement in the radiation susceptibility of devices having thinner gate oxides.

\subsubsection{Hole transport in $\mathrm{SiO}_{2}$}

The two-stage nature of time-dependent interface-trap build-up was first established by Winokur et al. [8991]. In this process as shown in Fig. 4.19, the first stage determines the saturated value of interface traps and occurs while holes move through the oxide and are subsequently trapped at the interface. The second stage, which begins after hole transport and trapping [92] and can continue for thousands of seconds, determines the time scale of build-up. In order to explain the time and electric field dependence of interface trap generation for MOSCs, McLean [93] suggested that, during the first stage, hydrogen ions $\left(\mathrm{H}^{+}\right)$are released in the bulk of the oxide as radiation-induced holes transport to the interface via polaron hopping. This involves the transfer of energy from the moving hole to the $\mathrm{SiO}_{2}$ lattice.

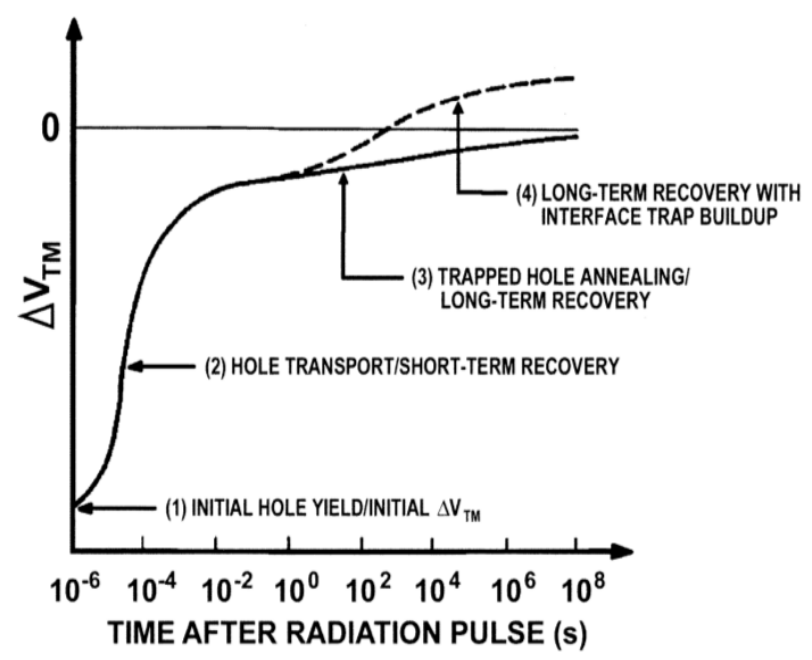

Figure 4.19: Time dependent threshold voltage recovery n-MOSFET following pulsed irradiation, indicating characteristic room temperature time regimes associated with various basic physical processes as well as various possible long-term responses. Figure taken from [73].

An increase in electric field increases the energy a hole imparts to the $\mathrm{SiO}_{2}$ lattice as it moves through the oxide, causing more $\mathrm{H}^{+}$to be released [94]. This in turn results in an increase in interface trap formation at higher electric fields. In addition, Shaneyfelt et al. [95] in order to explain an approximate $\mathrm{E}^{-1 / 2}$ field 
dependence of oxide- and interface-trap build-up, suggested the hole trapping/hydrogen transport (HT) ${ }^{2}$ model. In this model, for positive bias during irradiation, holes move to the $\mathrm{Si}_{-} \mathrm{SiO}_{2}$ interface and are trapped within $10 \mathrm{~nm}$ of the interface [96]. During their capture $\mathrm{H}^{+}$is released and expected to be the ratelimiting step. Time dependent studies have shown that hole trapping cannot be the rate-limiting step in the interface-trap build-up process [97]. Under negative bias, hole trapping events near the gate/ $\mathrm{SiO}_{2}$ interface similarly liberate $\mathrm{H}^{+}$, which may then drift to the $\mathrm{Si}_{-} \mathrm{SiO}_{2}$ interface and form interface traps.

\subsubsection{Annealing process}

The hole trap model $[98,99]$, presented in Fig. 4.20, is an attempt to explain both switching and nonswitching oxide traps with a single defect center. It assumes that holes are trapped at an oxygen deficient site near the interface to form an $\mathrm{E}^{\prime}$ center. Then the positively charged $\mathrm{Si}$ atom moves away from the uncharged Si atom and relaxes into a more planar configuration. The final separation distance between the two silicon atoms will depend upon the local bond strains. Then an electron tunneling from the silicon can be trapped on the neutral $\mathrm{Si}$ atom, eliminating the unpaired spin (and thus the $\mathrm{E}^{\prime} \mathrm{ESR}$ signal). If the Si-Si bond is reformed, then the defect is truly "annealed", and the charge state will not change with changes in bias. If the bond is not reformed, then the positive charge is merely compensated and a dipole is formed. If the bias is reversed, an electron could tunnel back into the silicon, returning the defect to its positively charged configuration. So far, the defect site is the same for both the switching and non-switching oxide traps. This model has been used to explain a number of effects, including permanent annealing of trapped holes, switching behavior of trapped holes, compensation effects, trapping and annealing of neutral electron traps, and "apparent" conversion of trapped holes to interface traps.

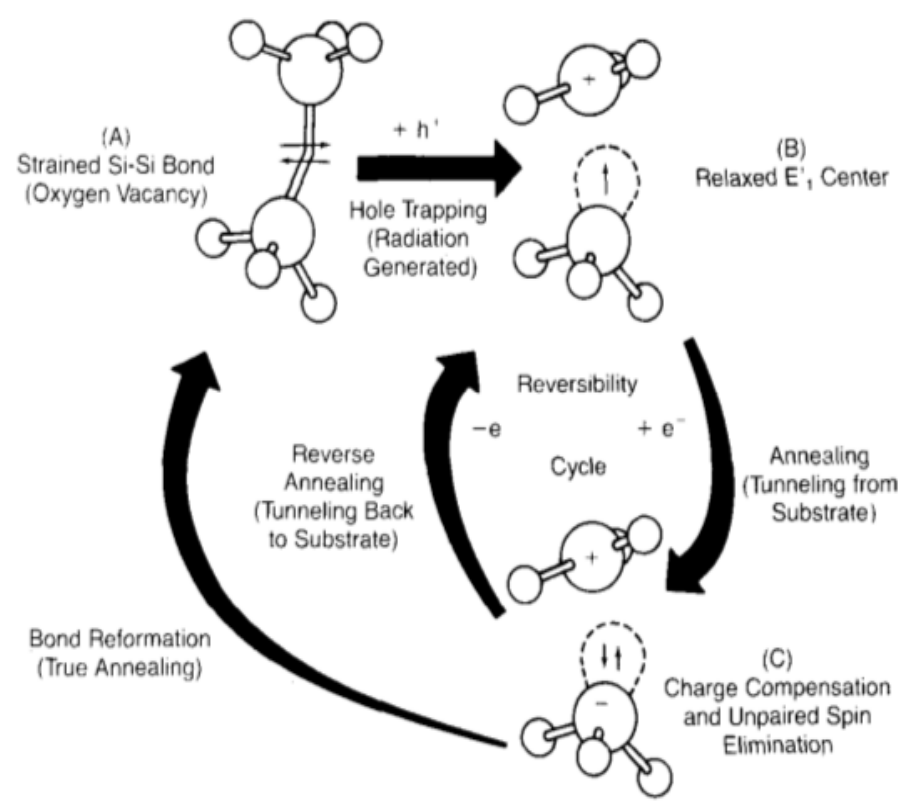

Figure 4.20: A model for the hole trapping [(a) to (b)] and de-trapping [(c) to (a)] processes are indicated, along with the intermediate compensation/reverse-annealing phenomenon [(b) to (c) and (c) to (b)]. Figure taken from [98]. 


\subsection{Effects of X-ray on MOS devices}

\subsubsection{Radiation-Induced Charge Neutralization-RICN effect}

According to Fleetwood et al. $[100,101]$ the peak charge neutralization rate is approximately equal to the rate of trapped-hole build-up under positive bias. This establishes an upper bound on the rate of radiationinduced charge neutralization and demonstrates that under peak neutralization conditions, the effective cross section for the capture of radiation-induced electrons by a filled hole trap is similar to the effective cross section for capture of a hole by an empty trap. Interface traps are found to build-up at approximately the same rate when the oxide electric field at the $\mathrm{Si}-\mathrm{SiO}_{2}$ interface is positive, regardless of the field direction in the bulk of the oxide. This suggests that near-interfacial hydrogen plays a key role in interface-trap build-up in MOS devices. As shown in Fig. 4.21 (a) threshold voltage shifts $\Delta V_{t h}$ are plotted as a function of X-ray dose for $\mathrm{n}$ - and p-MOSFETs with $t_{o x}=50 \mathrm{~nm}$ gate oxides.
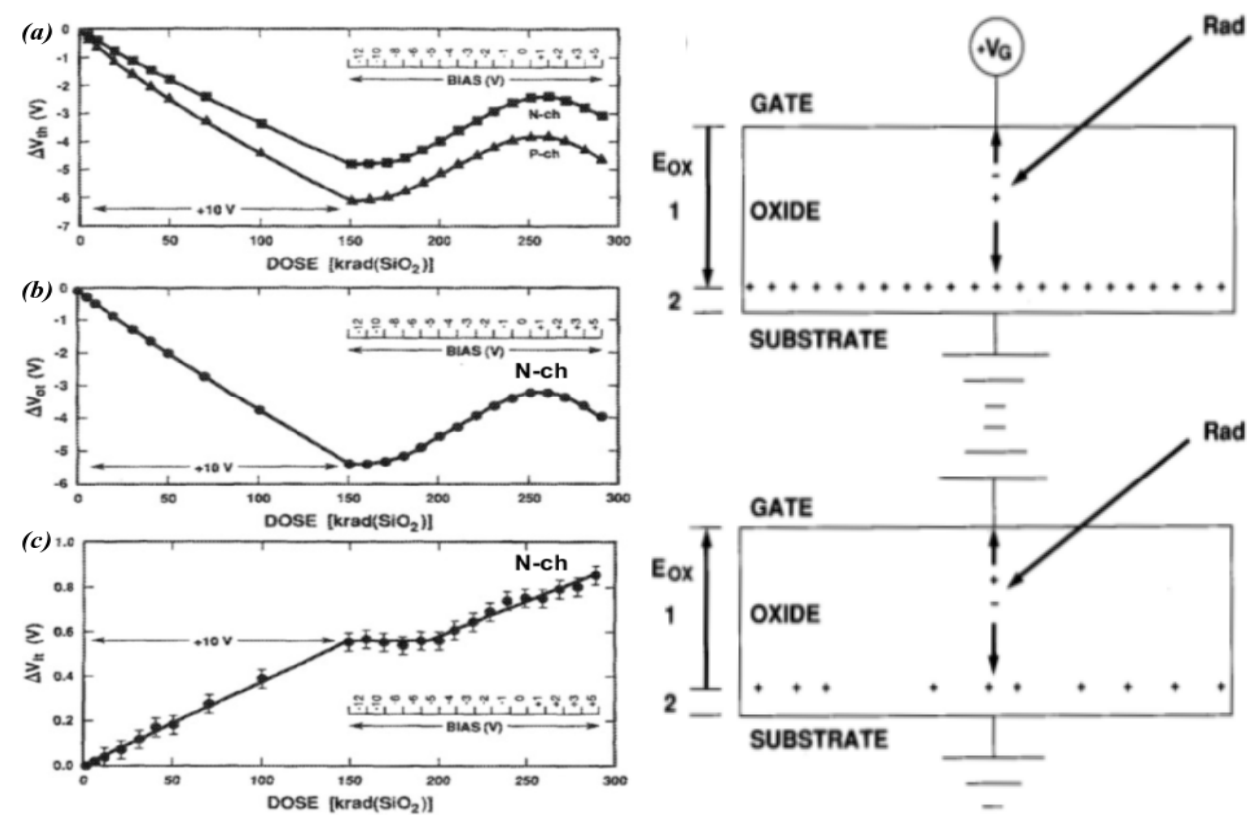

Figure 4.21: Threshold voltage shifts as a function of radiation dose and gate bias (a) for $n$ - and p-MOSFETs and threshold voltage shift, (b) due to oxide charge and (c) due to interface traps as a function of radiation dose and gate bias for n-MOSFET. The electric field in the oxide $E_{o x}$ for positive and zero gate voltage, is shown on the right. Figures taken from $[100,101]$.

For positive gate bias, Fig. 4.21 (right), the magnitude of $\Delta V_{t h}$ for both cases increases with X-ray dose, as expected. The offset between the curves occurs because interface traps shift the threshold voltage of a p-MOSFET negatively, and that of an n-MOSFET positively [102-104]. In addition, Figs. 4.21 (b) and (c) show $\Delta V_{o x}$ and $\Delta V_{i t}$ as a function of X-ray dose for the n-MOSFET. Comparing the Figs. 4.21 (a) and (b) the changes in threshold voltage are caused by the build-up and neutralization of the fixed oxide charge. Ionizing radiation produces electron-hole pairs in $\mathrm{SiO}_{2}$. At large electric fields $\approx 70 \%-90 \%$ of these pairs escape geminate recombination [105]. Holes execute a much slower random walk toward the Si substrate [92]. At constant positive bias, these holes are annealed, and/or "compensated" by electrons that tunnel into the oxide and become trapped nearby (rebound effect) [106, 107], over times ranging from milliseconds to 
years, depending on the energy of the traps and their distances from the interface. When the bias is changed from $+10 \mathrm{~V}$ to $-12 \mathrm{~V}$ at $150 \mathrm{krad}$, the direction of electron and hole transport processes are reversed, and "conventional" trapped-hole neutralization via tunneling processes at the $\mathrm{Si}_{-} \mathrm{SiO}_{2}$ interface is suspended. Holes now exit into the gate, or are trapped so close to the gate that they shift the threshold voltage negligibly, and electrons move toward the $\mathrm{Si}-\mathrm{SiO}_{2}$ interface. If an electron encounters a hole trapped near the $\mathrm{Si}-\mathrm{SiO}_{2}$ interface, the electron may become trapped nearby and neutralize the charge of the hole, or recombine with the trapped hole directly, reducing the $\Delta V_{o x}$ in either case. The effective charge neutralization rate between $-6 \mathrm{~V}$ and $-1 \mathrm{~V}$ (180 krad to $240 \mathrm{krad}$ ), as shown by the slope in Fig. 4.21 (b), is approximately constant and equal to the rate of hole build-up from 0 to $150 \mathrm{krad}$ under $+10 \mathrm{~V}$ bias. This equality of rates implies that, under conditions of peak neutralization, the effective cross section for radiation-induced electron capture by a positively charged oxide trap is similar to the effective hole capture cross section of an empty trap [108]. Interface trap build-up through the charge neutralization process, is presented in Fig. 4.21 (b), the values of $\Delta V_{i t}$ for the n-MOSFET of Fig. 4.21 (a). From 0 to $150 \mathrm{krad}$, interface traps build-up linearly with dose, as expected for this bias. Following the switch to negative bias, no interface trap build-up is observed for biases below $-4 \mathrm{~V}$. However interface traps clearly are building-up for biases between $-4 \mathrm{~V}$ and $0 \mathrm{~V}$. Assuming that $\mathrm{H}^{+}$ions are responsible for the interface trap build-up [89], from $-12 \mathrm{~V}$ to $-5 \mathrm{~V}$, the applied bias is sufficiently large that a negative field exists across the entire oxide, and no interface trap build-up is observed. At a bias of $-4 \mathrm{~V}$, however, the field due to the fixed oxide charge exceeds that of the applied gate bias, and the field at the $\mathrm{Si}_{-} \mathrm{SiO}_{2}$ interface is positive, Fig. 4.21 (c). If $\mathrm{H}^{+}$ion drift is responsible for the interface trap build-up, this result implies that the $\mathrm{H}^{+}$ions responsible for creating the interface traps must be liberated between the point of field reversal in the oxide and the substrate, in the region of the oxide where the electric field is positive. The rate of build-up between $-4 \mathrm{~V}$ and $0 \mathrm{~V}$, Fig. 4.21 (right), is similar to the rate of build-up under positive bias may also suggest that near interfacial hydrogen plays a key role in all of the observed interface trap build-up.

\subsubsection{Rebound effect}

The physical mechanisms that produce "rebound" or "recovery" effects have been identified in Refs. [106, 109, 110]. The positive increase in threshold voltage during a bias anneal is due to annealing of oxide trapped charge. Rebound can be predicted by measuring the contribution to the threshold voltage from radiation-induced interface states immediately after irradiation. In particular after an initial negative decrease in the threshold voltage of an n-MOSFET during irradiation, under a positive bias the threshold voltage may increase to values well above the pre-irradiation threshold voltage. In Fig. 4.22 (a) is presented the threshold voltage, $V_{t h}$, of a n-MOSFET with $t_{o x}=45 \mathrm{~nm}$, during irradiation and during a bias anneal at room temperature and at $125^{\circ} \mathrm{C}$ [106]. The gate voltage was, $V_{\text {gate }}=10 \mathrm{~V}$, and the drain-source voltage was, $V_{d s}=0 \mathrm{~V}$ during both irradiation and anneal. The threshold voltage shows the rebound effect. During irradiation ( $<1$ hour) the threshold voltage decreased to approximately zero volts. During the bias anneal the threshold voltage increased to over $3.5 \mathrm{~V}$ at $125^{\circ} \mathrm{C}$. The room temperature data, also tending to the same value. The contribution of interface traps, $\Delta V_{N i t}$, and oxide charge $\Delta V_{N o x}$ are also shown in Fig. 4.22 (a). During irradiation $\Delta V_{N i t}$ increased by approximately $2.5 \mathrm{~V}$ and $\Delta V_{N o x}$ decreased by approximately $3.5 \mathrm{~V}$, the net threshold voltage shift being $-1.0 \mathrm{~V} . \Delta V_{N i t}$ did not change significantly during the bias anneal. In approximately 100 hours of bias anneal $\Delta V_{N o x}$ was completely annealed at $125^{\circ} \mathrm{C}$. At this point, the threshold 
voltage shift is due entirely to interface state charge. The room temperature data also indicates an annealing of the oxide charge, tending to its pre-irradiation value.

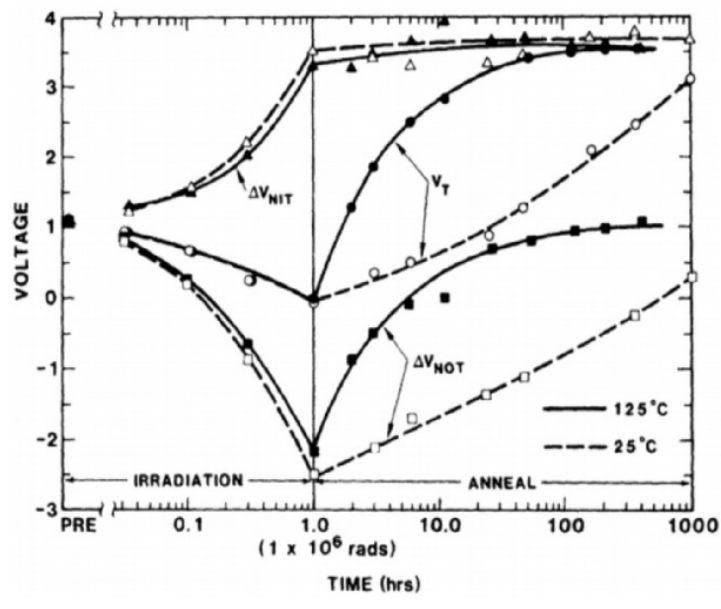

(a)

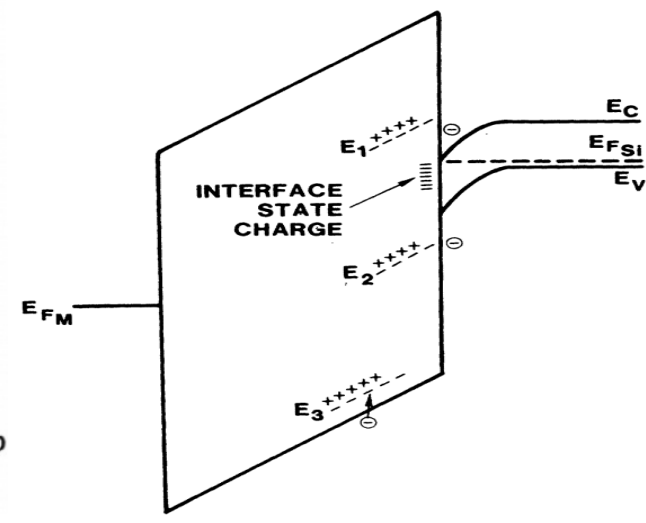

(b)

Figure 4.22: (a) Threshold voltage shift of an n-MOSFET during irradiation and anneal separated into the shift due to interface traps and oxide charge. $V_{\text {gate }}=10 \mathrm{~V}$ during irradiation and anneal. (b) Energy band diagram of an n-MOSFET with a positive gate bias. Three possible oxide trap locations $E_{1}, E_{2}$ and $E_{3}$ are shown. Figures taken from [106].

Mechanisms for rebound An energy band diagram of an n-MOSFET is shown in Fig. 4.22 (b) for a positive gate bias. For a positive gate bias the Fermi level is near the silicon conduction band at the silicon surface. The acceptor type interface traps in the upper half of the silicon bandgap below the Fermi level will be negatively charged $[111,112]$. The donor type interface traps in the lower half of the bandgap will be neutral. According to Ref. [96] the fixed oxide charge is believed to be an $E^{\prime}$ center, located within 5 $\mathrm{nm}$ of the $\mathrm{Si}_{-} \mathrm{SiO}_{2}$ interface. As shown in Fig. 4.22 (b), two possible locations of the oxide charge near the $\mathrm{Si}-\mathrm{SiO}_{2}$ interface are the levels $E_{1}$ and $E_{2} . E_{1}$ is located above the silicon conduction band and $E_{2}$ below the silicon valence band. A third location in energy, is the energy level $E_{3}$ located above the oxide valence band. It appears that electrons either from the oxide valence band or from silicon are neutralizing the oxide trapped charge. The time and temperature dependence of rebound can be accounted by a thermally assisted tunneling process. For this case electrons can tunnel either from the silicon conduction band into trap $E_{1}$ or from the silicon valence band into trap $E_{2}$ [113]. If an electron tunnels into the oxide it is captured by the oxide trap. The conduction electrons will have a temperature dependence given by Fermi-Dirac statistics. As it is stated in Ref. [106], a bias dependence of the rate of oxide trapped charge annealing will be seen if the energy location of the oxide trap is that given by $E_{1}$. The number of electrons in the silicon conduction band will increase with gate bias. The tunneling rate increases with the number of surface electrons. A bias dependence will be seen for electrons in the silicon valence band tunneling into trap level $E_{2}$ if the oxide trap extends into the region nearby to the silicon bandgap. Decreasing the bias will increase the fraction of the traps no longer accessible to valence band electrons. For trap $E_{3}$, located above the oxide valence band a thermal activation energy would be measured directly [114]. Independent of the energy position of the oxide traps in the silicon dioxide bandgap, a mechanism is present where electrons are tunneling into the oxide traps and neutralize the charge. When the oxide charge is completely neutralized, the net threshold voltage 
shift is due to charge from the interface traps. For a negative bias voltage after irradiation, the energy level of the oxide trap is raised above the silicon conduction band edge decreasing the probability of occupancy by an electron. The weakly bound electron on the oxide trap defect can escape from the trap and tunnel back into the silicon. Furthermore the p-MOSFETs do not show a rebound effect after irradiation [106,115]. The threshold voltage of a p-MOSFET decreases negatively during irradiation. During a bias anneal the threshold voltage increases positively, but is still more negative that the pre-irradiation threshold value. The Fermi level for a p-MOSFET is near the silicon valence band during a threshold voltage measurement. The donor type interface traps in the lower half of the silicon bandgap with energies above the Fermi level will be positively charged. The acceptor type interface traps in the upper half of the silicon bandgap will be neutral. The oxide trapped charge is still positive. Both $\Delta V_{N i t}$ and $\Delta V_{N o x}$ are negative. During a positive bias anneal the oxide trapped charge for a p-MOSFET is also neutralized. However, since $\Delta V_{N i t}$ is negative, the threshold voltage after a bias anneal will be still be more negative than the pre-irradiation threshold voltage. Finally, the rebound effect is temperature and bias dependent. Increasing temperature increases the rate of oxide trapped charge annealing. Decreasing the bias causes an incomplete annealing of the oxide trapped charge. The rebound effect can be minimized by using process techniques that minimize the creation of radiation-induced interface traps.

\subsection{Effects on high-ohmic MOSC and GCD}

\subsubsection{Experimental observations}

In order to investigate X-ray radiation damage parameters on high-ohmic silicon sensors like the oxide charge density and the surface current density MOSCs and GCDs are designed on the same wafer together with the sensors. On the AGIPD wafer has been designed and fabricated a MOSC, shown in Fig. 4.23 (a), has a circular shape with a diameter of $1.5 \mathrm{~mm}$, surrounded by a $100 \mu \mathrm{m}$ wide gate ring.

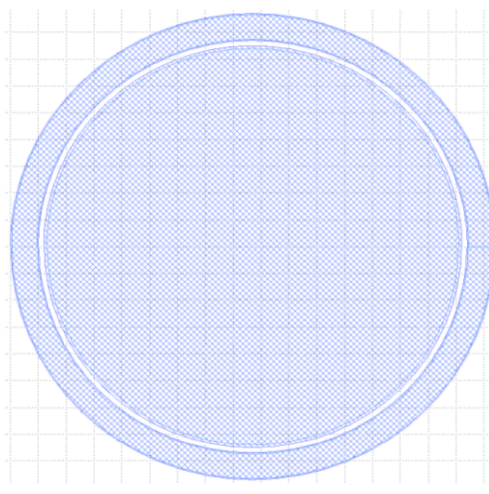

(a)

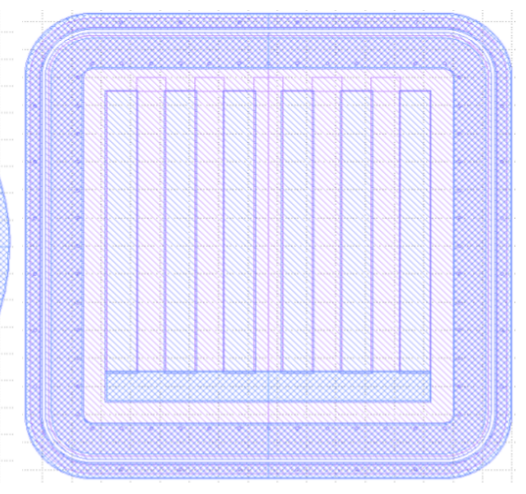

(b)

Figure 4.23: Masks of (a) MOS Capacitor and (b) Gate Controlled Diode designed and fabricated on the AGIPD wafer.

The intersection of the $\mathrm{C}-\mathrm{V}$ curve with $C_{f b}$ is the flatband voltage $V_{f b}$ and the effective oxide charge density $N_{o x}^{\text {eff }}$ (fixed oxide charge + interface traps) is calculated from 


$$
N_{o x}^{e f f}=\frac{C_{o x}\left(-V_{f b}+\phi_{m s}\right)}{q_{0}}
$$

and $\phi_{m s}=-0.42 \mathrm{~V}$ for the Al-Si work function difference. The $\mathrm{C}-\mathrm{V}$ measurement on the MOSC before irradiation shows a flatband voltage $V_{f b}$ of $-0.40 \mathrm{~V}$. The difference between $V_{f b}$ and $\phi_{m s}$ indicates a negative charge in the insulating layer with an equivalent density of $1.8 \cdot 10^{10} \mathrm{~cm}^{-2}$ at the $\mathrm{Si}_{-} \mathrm{SiO}_{2}$ interface. The geometry of the GCD is shown in Fig. 4.23 (b). It is a finger-like structure with 6 vertical and 1 horizontal fingers surrounded by a diode. The width of the fingers is $100 \mu \mathrm{m}$ each and the lengths for the 6 vertical and 1 horizontal fingers are $1000 \mu \mathrm{m}$ and $1100 \mu \mathrm{m}$, respectively. The gate area is $7.1 \cdot 10^{-3} \mathrm{~cm}^{2}$ and the surface current density, $J_{\text {surf }}$, extracted from the I-V measurement before irradiation is $2.0 \mathrm{nA} / \mathrm{cm}^{2}$. During the irradiations, no voltages were applied to the electrodes of the MOSC and GCD. The C-V and I-V measurements were performed within 1 hour after each irradiation. In Fig. 4.24 (a) and (b) are presented $\mathrm{C}-\mathrm{V}$ and I-V curves from the MOSC and GCD before and after annealing at $80{ }^{\circ} \mathrm{C}$ for 10 minutes in order to obtain reproducible results.

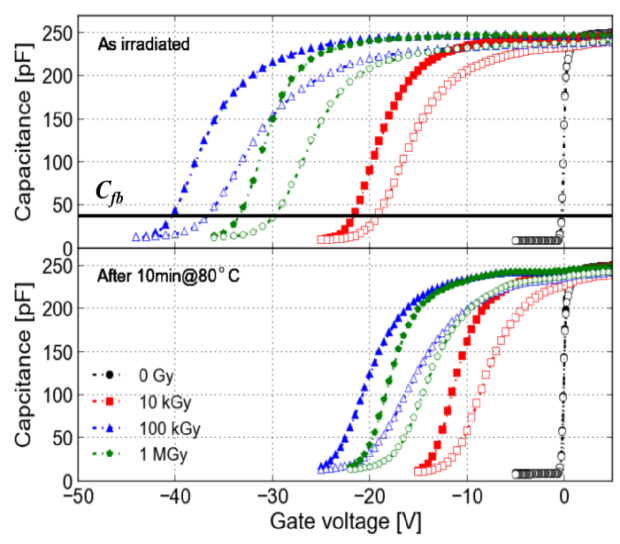

(a)

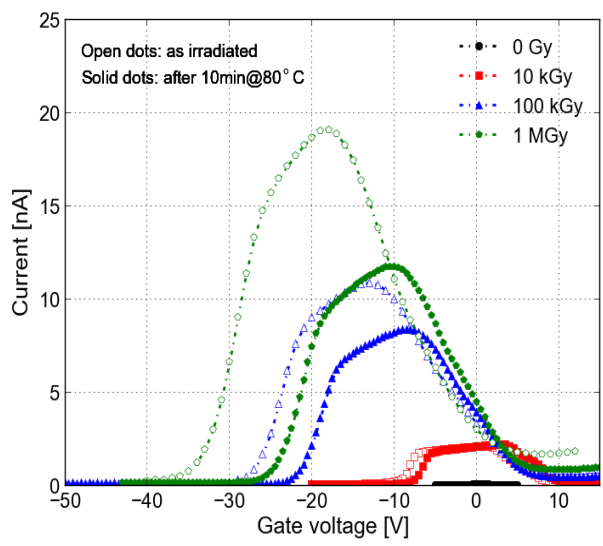

(b)

Figure 4.24: (a) C-V curves of the MOSC and (b) I-V curves of the GCD.

From the $\mathrm{C}-\mathrm{V}$ curves of the irradiated MOSC, a maximal voltage shift of $45 \mathrm{~V}$ has been found for $100 \mathrm{kGy}$. However, after annealing at $80{ }^{\circ} \mathrm{C}$ for 10 minutes, $\mathrm{C}-\mathrm{V}$ curves from $10 \mathrm{MGy}$ show a maximal voltage shift, which may indicate different annealing time constants for different doses. Not only the annealing of oxidetrapped charges, but also the removal of free carriers in the $\mathrm{SiO}_{2}$ produced by prompt X-rays, which also can shift the flatband voltage of the MOSC. From the I-V curves of the GCD, the maximal surface current was found at $1 \mathrm{MGy}$ and it decreases for doses above $1 \mathrm{MGy}$. In Fig. 4.25 (a) the $N_{\text {ox }}^{\text {eff }}$ and Fig. 4.25 (b) the $J_{\text {surf }}$, extracted from the MOSC and GCD after annealing at $80{ }^{\circ} \mathrm{C}$ for 10 minutes and shown as black triangles, are plotted as a function of dose and compared to previous measurement results $[8,116]$. It is observed that $N_{o x}^{e f f}$ and $J_{\text {surf }}$ for the AGIPD wafer are consistent with the results obtained from the structures produced by the same vendor (SINTEF) with a different oxide thickness, shown in yellow. $N_{o x}^{\text {eff }}$ and $J_{\text {surf }}$ saturate at a dose between $100 \mathrm{kGy}$ and $1 \mathrm{MGy}$ for irradiation without bias voltage, and the saturation values are $(2-2.5) \times 10^{12} \mathrm{~cm}^{-2}$ and $1.7 \mu \mathrm{A} / \mathrm{cm}^{2}$. 


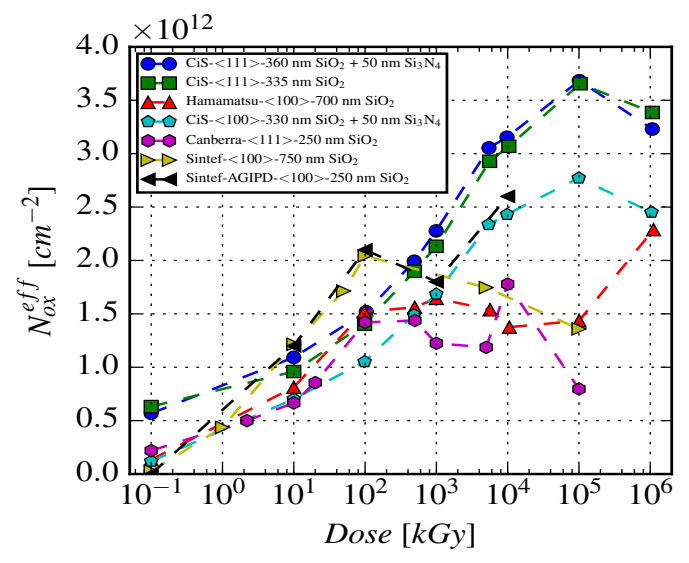

(a)

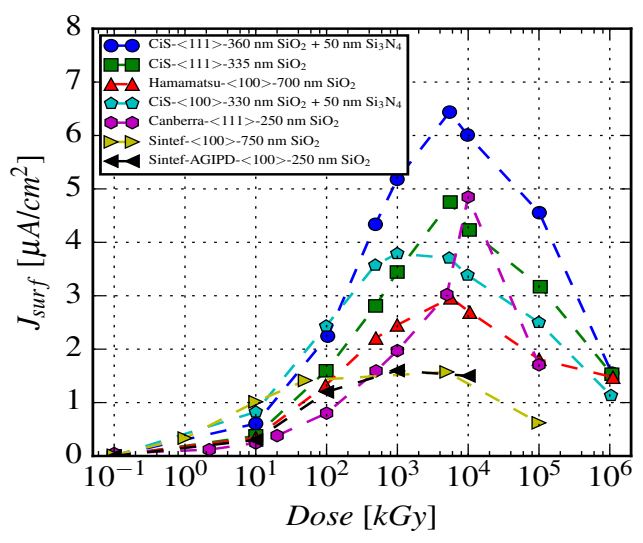

(b)

Figure 4.25: (a) $N_{o x}^{e f f}$ and (b) $J_{\text {surf }}$ as a function of X-ray dose. Data taken from $[4,8]$.

\subsubsection{Electric field dependence of $N_{o x}^{e f f}$ on high-ohmic MOSC}

Field-enhanced injection of positive oxide charges has been presented in Refs. [117, 118]. C-V curves of circular MOSCs have been measured at frequencies between $100 \mathrm{~Hz}$ and $2 \mathrm{MHz}$ for a $<100>$ MOSC and $<111>$ MOSC non-irradiated and irradiated to $1 \mathrm{GGy}$ without bias voltage applied during irradiation. The following biasing cycle for the gate voltage, $V_{\text {gate }}$, has been followed: set $V_{\text {gate }}$ to $V_{i n v}$ in inversion conditions, remain at $V_{i n v}$ for the time interval $t_{\text {bias }}$, ramp $V_{\text {gate }}$ from inversion to accumulation (forward ramp), ramp $V_{\text {gate }}$ back to $V_{i n v}$ (reverse ramp), start a new cycle with a different $t_{\text {bias }}$ value. The $t_{\text {bias }}$ are chosen 0,30 , 60 , and $120 \mathrm{~min}$. In order to check if the charge injection saturates at long $t_{\text {bias }}$, additional measurements have been performed up to $t_{\text {bias }} \approx 15 \mathrm{~h}$ for the irradiated MOSCs. For the study of the dependence of the charge injection on the electric field at the $\mathrm{Si}_{-} \mathrm{SiO}_{2}$ interface, three different values for $V_{i n v}$ in increasing order were chosen. The corresponding field values were between 0.3 and $1.5 \mathrm{MV} / \mathrm{cm}$. The data for the

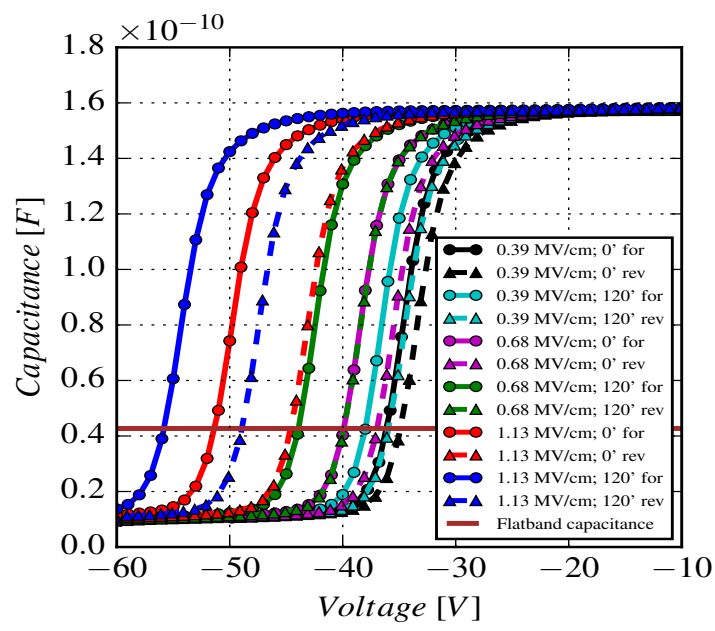

Figure 4.26: $\mathrm{C}-\mathrm{V}$ curves at $10 \mathrm{kHz}$ for the irradiated $<111>$ MOSC measured at $-20{ }^{\circ} \mathrm{C}$ for the 3 values of $V_{i n v}$ and $t_{\text {bias }}=0$ and $120 \mathrm{~min}$. The results for the forward $(f)$ and for the reverse $(r)$ voltage ramping are shown. The horizontal line gives the value of the flatband capacitance. Data taken from [117]. 
non-irradiated MOSCs were recorded at $20{ }^{\circ} \mathrm{C}$ and a relative humidity below $5 \%$. The irradiated MOSCs were measured at $-20{ }^{\circ} \mathrm{C}$ to prevent annealing. It has been verified for the non-irradiated MOSC that the results for both temperatures agree. Fig. 4.26 shows the $10 \mathrm{kHz} \mathrm{C-V} \mathrm{curves} \mathrm{for} \mathrm{the} \mathrm{irradiated}<111>$ MOSC. The horizontal line indicates the flatband capacitance. With increasing $t_{b i a s}$ and with increasing $V_{i n v}$ the $\mathrm{C}-\mathrm{V}$ curves shift to more negative voltages. It is observed that the forward $\mathrm{C}-\mathrm{V}$ curve obtained when changing the voltage from inversion to accumulation is shifted to more negative voltages compared to the reverse $\mathrm{C}-\mathrm{V}$ curve. It is defined as $N_{o x, 0}^{e f f}$ the value of $N_{o x}^{e f f}$ determined from the forward C-V curve at the lowest $V_{i n v}$ and for $t_{\text {bias }}=0 \mathrm{~min}$, and assume that it approximately corresponds to the initial oxide charge density from the production process and the X-ray radiation damage, if applicable. The difference $\Delta N_{o x}^{e f f}=N_{o x}^{e f f}-N_{o x, 0}^{e f f}$

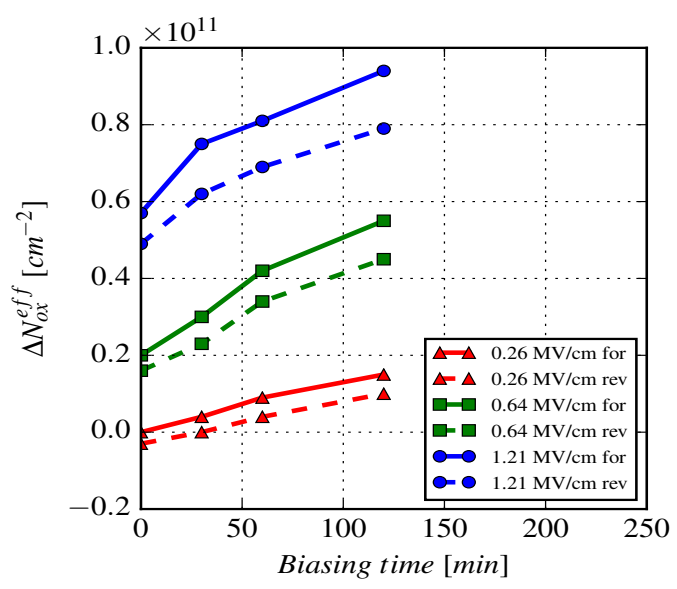

Figure 4.27: $\Delta N_{o x}^{e f f}$ as a function of biasing time for the different inversion gate voltages $V_{i n v}$. Both forward and reverse $\mathrm{C}-\mathrm{V}$ curves measured at $10 \mathrm{kHz}$ are shown for non-irradiated $<111>\mathrm{MOSC}$ at $20^{\circ} \mathrm{C}$. Data taken from [117].

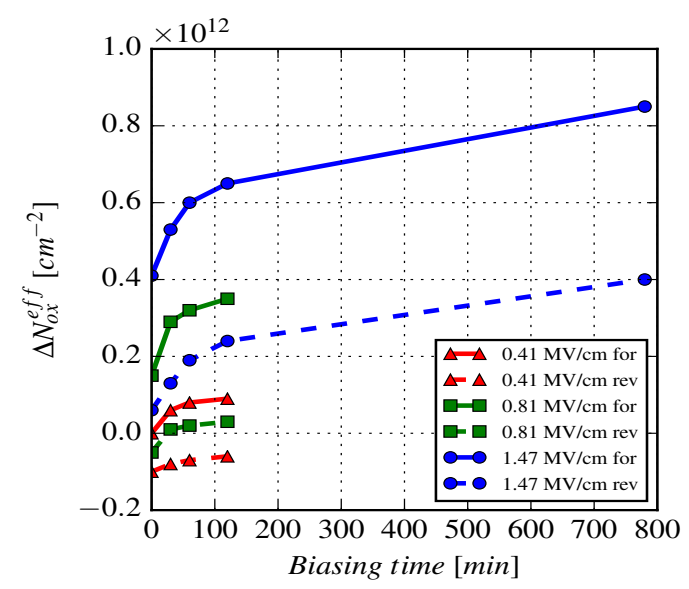

(a)

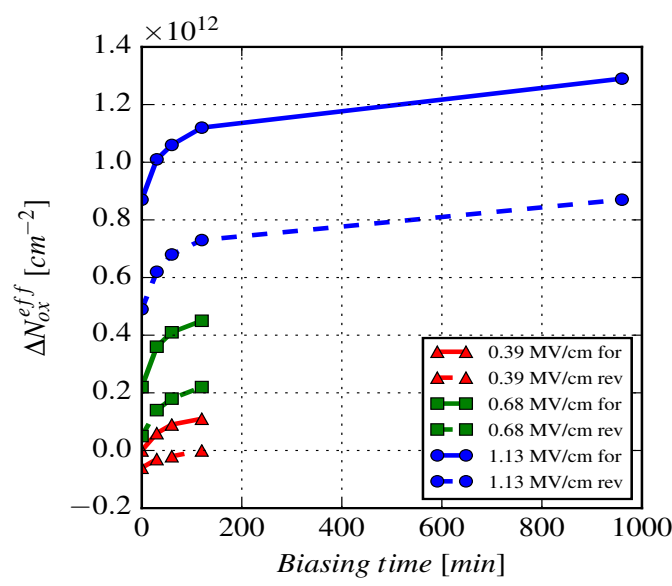

(b)

Figure 4.28: $\Delta N_{o x}^{e f f}$ as a function of biasing time for the different inversion gate voltages $V_{i n v}$. Both forward and reverse $\mathrm{C}-\mathrm{V}$ curves measured at $10 \mathrm{kHz}$ are shown for, (a) irradiated $<100>$ MOSC at $-20{ }^{\circ} \mathrm{C}$ and (b) irradiated $<111>\mathrm{MOSC}$ at $-20^{\circ} \mathrm{C}$. Data taken from [117].

is assumed to be the additional positive charge injected from the $\mathrm{Si}$ into the $\mathrm{SiO}_{2}$. The electric field at the $\mathrm{Si}_{-} \mathrm{SiO}_{2}$ interface is calculated using $E_{\text {field }}=\left|V_{\text {gate }}-V_{f b}\right| / t_{o x}$, with the oxide thickness $t_{o x}$. The $\Delta N_{o x}^{\text {eff }}$ 
as a function of $t_{\text {bias }}$, for the forward $\Delta N_{o x, \text { forw }}^{\text {eff }}$ and reverse $\Delta N_{o x, \text { rev }}^{\text {eff }}$, of $\mathrm{C}-\mathrm{V}$ measurements at $10 \mathrm{kHz}$ is presented in Fig. 4.27 for the non-irradiated $<111>$ MOSC. For the irradiated $<100>$ and $<111>$ MOSCs in Figs. 4.28 (a) and (b) respectively. The results for $100 \mathrm{kHz}$ are very similar. Table 4.3 presents the values of $\Delta N_{o x, \text { forw }}^{e f f}$ and $\Delta N_{o x}^{e f f}$ rev for $t_{\text {bias }}=120 \mathrm{~min}$ and the electric field values corresponding to the $V_{i n v}$ values of the measurements. The $N_{o x, 0}^{e f f}$ for $<111>0$ Gy is $5.5 \cdot 10^{11} \mathrm{~cm}^{-2}$, for $<100>1 \mathrm{GGy}$ is $15 \cdot 10^{11} \mathrm{~cm}^{-2}$ and for $<111>1 \mathrm{GGy}$ is $21 \cdot 10^{11} \mathrm{~cm}^{-2}$.

Table 4.3: The change of oxide charge density $\Delta N_{o x}^{\text {eff }}$ due to charge injection at the $\mathrm{Si}_{-} \mathrm{SiO}_{2}$ interface for different electric fields after the biasing time $t_{\text {bias }}=120 \mathrm{~min}$, where $f$ refers to the voltage ramping from inversion to accumulation, and $r$ for the opposite ramping.

\begin{tabular}{|c|c|c|c|c|c|c|c|c|c|}
\hline $\begin{array}{l}\text { Orientation } \\
\text { Dose }\end{array}$ & & $\begin{array}{l}<111> \\
0 \mathrm{~Gy}\end{array}$ & & & $\begin{array}{l}<100> \\
1 \mathrm{GGy}\end{array}$ & & & $\begin{array}{l}<111> \\
1 \mathrm{GGy}\end{array}$ & \\
\hline$E[\mathrm{MV} / \mathrm{cm}]$ & 0.26 & 0.64 & 1.28 & 0.41 & 0.81 & 1.47 & 0.39 & 0.68 & 1.13 \\
\hline$\Delta N_{o x, f}^{e f f}\left[\mathrm{~cm}^{-2}\right]$ & $0.15 \cdot 10^{11}$ & $0.55 \cdot 10^{11}$ & $0.94 \cdot 10^{11}$ & $0.94 \cdot 10^{11}$ & $3.5 \cdot 10^{11}$ & $6.5 \cdot 10^{11}$ & $1.1 \cdot 10^{11}$ & $4.5 \cdot 10^{11}$ & $11.2 \cdot 10^{11}$ \\
\hline$\Delta N_{o x, r}^{e f f}\left[\mathrm{~cm}^{-2}\right]$ & $0.10 \cdot 10^{11}$ & $0.45 \cdot 10^{11}$ & $0.79 \cdot 10^{11}$ & $-0.6 \cdot 10^{11}$ & $0.3 \cdot 10^{11}$ & $2.3 \cdot 10^{11}$ & $0.0 \cdot 10^{11}$ & $2.2 \cdot 10^{11}$ & $7.3 \cdot 10^{11}$ \\
\hline
\end{tabular}

In all cases $\Delta N_{o x, f o r w}^{e f f}>0$ which is evidence for field-induced charge injection. The values increase with $t_{\text {bias }}$, with a typical time constant of about $30 \mathrm{~min}$, and saturation values are reached after about $120 \mathrm{~min}$. However for the non-irradiated MOSC the effects are at most $20 \%$ of the initial $N_{o x, 0}^{e f f}$ and for the irradiated they are as high as $50 \%$. For the $<111>$ the effects are always bigger than the $<100>$ MOSC. The significant difference between the reverse and forward ramp, is interpreted as evidence for a short-term discharge of positively charged states when the interface is in accumulation. It appears that the densities of discharged states, $\Delta N_{o x, \text { forw }}^{\text {eff }}-\Delta N_{o x, \text { rev }}^{\text {eff }}$, are similar for the $<100>$ and the $<111>$ MOSC at the same values of electric field and $t_{\text {bias }}$. So far, the values of $N_{o x, 0}^{e f f}$ and $\Delta N_{o x, f o r w}^{e f f}$ are significantly larger for the $<111>$ than for the $<100>$ MOSC. The observed injection of positive charges has to be taken into account when $\mathrm{C}-\mathrm{V}$ measurements on MOSCs fabricated on $\mathrm{n}-\mathrm{Si}$ are used to determine the $N_{o x}^{\text {eff }}$ at the $\mathrm{Si}_{-} \mathrm{SiO}_{2}$ interface as a function of ionizing dose. According to TCAD simulations [6] of $\mathrm{p}^{+} \mathrm{n}$ sensors as a function of X-ray dose [4], there is only a small region at the metal overhangs where the electric field, Fig. 4.29 (a), points into the $\mathrm{SiO}_{2}$.

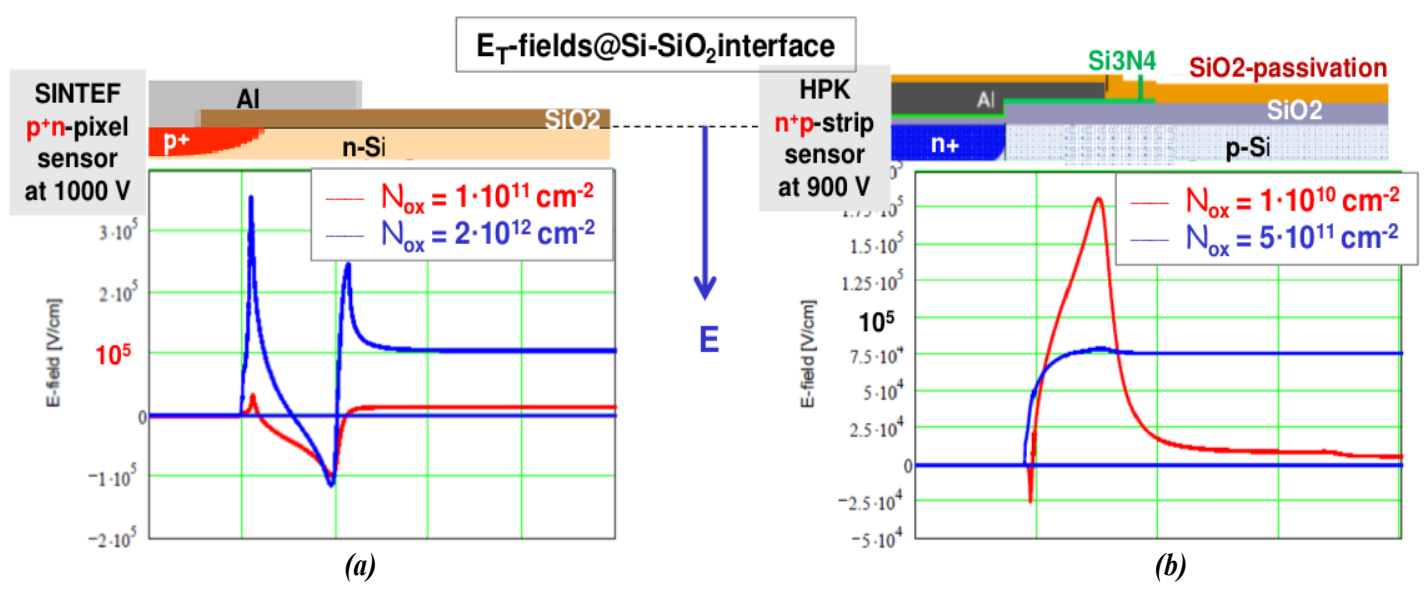

Figure 4.29: Electric field at the $\mathrm{Si}_{-} \mathrm{SiO}_{2}$ interface. Figure taken from [119].

The simulated field components normal to the $\mathrm{Si}_{-} \mathrm{SiO}_{2}$ interface are below $500 \mathrm{kV} / \mathrm{cm}$. For $\mathrm{n}^{+} \mathrm{p}$ sensors the electric field at the $\mathrm{Si}_{-} \mathrm{SiO}_{2}$ interface, Fig. 4.29 (b), is either close to zero or points into the $\mathrm{Si}$, once the positive oxide charge density exceeds the charge density due to the $\mathrm{p}^{+}$implant used to isolate the $\mathrm{n}^{+}$ implants of the segmented electrodes [25]. 



\section{X-ray dose and $E_{\text {field }}$ dependence of $N_{o x}^{e f f}$}

In this chapter is presented the measurement of the $E_{\text {field }}$ and time dependence of the $N_{o x}^{\text {eff }}$ of the $\mathrm{Si}-\mathrm{SiO}_{2}$ system as a function of X-ray dose using high-ohmic $n$ - and p-MOSFETs, fabricated on the same wafer with silicon sensors. The MOSFET structures are built on $<100>$ and $<111>$ bulk Si with oxide thickness $700 \mathrm{~nm}$ and $250 \mathrm{~nm}$ respectively.

\subsection{High-ohmic p- and n-MOSFETs irradiated with $E_{\text {field }}$}

The surface radiation damage of the $\mathrm{Si}_{-} \mathrm{SiO}_{2}$ interface and of $\mathrm{SiO}_{2}$ grown on high-ohmic n- and p-type $\mathrm{Si}$, as used for the fabrication of segmented silicon sensors, has been investigated. Circular p- and n-MOSFETs, biased in inversion and accumulation at an electric field in the $\mathrm{SiO}_{2}$ of about $500 \mathrm{kV} / \mathrm{cm}$, have been irradiated by $\mathrm{X}$-rays up to a dose of about $17 \mathrm{kGy}\left(\mathrm{SiO}_{2}\right)$ in different irradiation steps. Before and after each irradiation, the gate voltage has been cycled from inversion to accumulation conditions and back, and the threshold voltage of the MOSFETs and the hole and electron mobility at the $\mathrm{Si}_{-} \mathrm{SiO}_{2}$ interface determined. From the threshold voltage, the effective oxide charge density, $N_{o x}^{e f f}$, is calculated. The measurement of the drainsource current during the irradiation allows the study of the change of the oxide charge density during irradiation. Results on the dose dependence of the effective oxide charge density, the charging up and discharging of border traps when changing the gate voltage, and the hole and electron mobility at the Si$\mathrm{SiO}_{2}$ interface are presented.

\subsubsection{The p-MOSFET irradiated with $E_{\text {field }}$ pointing from the $\mathrm{Si}$ into the $\mathrm{SiO}_{2}$}

In this section is presented how $N_{o x}^{e f f}$ depends on the X-ray dose and on the direction of the electric field in the $\mathrm{SiO}_{2}$. In addition, the dependence of the hole mobility at the $\mathrm{Si}_{-} \mathrm{SiO}_{2}$ interface on $\mathrm{X}$-ray dose and electric field in the $\mathrm{SiO}_{2}$ is determined. A circular p-MOSFET fabricated on n-doped $\mathrm{Si}$ with $<111>$ crystal orientation has been irradiated by $\mathrm{X}$-rays to a dose of $\approx 17 \mathrm{kGy}\left(\mathrm{SiO}_{2}\right)$ with increasing irradiation steps, and the time dependence of the drain-source current, $I_{d s}(t)$, has been measured. During the irradiations the MOSFET has been biased in strong inversion, resulting in an electric field in the $\mathrm{SiO}_{2}$ of $500 \mathrm{kV} / \mathrm{cm}$ pointing from the $\mathrm{Si}$ into the $\mathrm{SiO}_{2}$ [120]. In Ref. [121] the electric fields in silicon strip detectors have been calculated using TCAD simulations taking into account surface radiation damage, and it is concluded that $500 \mathrm{kV} / \mathrm{cm}$ can be assumed as an upper limit for the electric field at the $\mathrm{Si}_{-} \mathrm{SiO}_{2}$ interface of silicon sensors, also shown in Ch. 4. 
From the dependence $I_{d s}\left(V_{\text {gate }}\right)$ of the MOSFET, which was measured by cycling the gate voltage $V_{\text {gate }}$ from deep inversion to accumulation and back, the threshold voltage $V_{t h}$ and the hole mobility are obtained. From $V_{t h}, N_{o x}^{e f f}$ is derived. The $I_{d s}\left(V_{\text {gate }}\right)$ curves also allow to determine at constant $V_{\text {gate }}$ the time dependence of $V_{t h}(t)$ from $I_{d s}(t)$ before, during and after the X-ray irradiation.

Similar measurements on p-MOSFET with a reversed electric field direction during the irradiations, and on n-MOSFETs with both field directions, are presented in next section. The final aim of the study is to provide parametrizations of the effective oxide charge densities and of the mobilities of holes and electrons at the $\mathrm{Si}-\mathrm{SiO}_{2}$ interface as a function of ionizing dose and direction of the electric field at the maximum value expected for segmented silicon sensors, which can be used in TCAD simulations for designing radiation tolerant segmented silicon sensors.

Test structure For the present study, a circular p-MOSFET, fabricated by Canberra on $<111>$ n-type FloatZone (FZ) Si with a bulk doping of $N_{d}=6.2 \cdot 10^{11} \mathrm{~cm}^{-3}$, has been used. Fig. 5.1 shows a cross section and a top view of the p-MOSFET. The width over length ratio $W / L=2 \pi / \ln \left(r_{2} / r_{1}\right) \approx 9.06$, where $r_{1}=250 \mu \mathrm{m}$ is the inner and $r_{2}=500 \mu \mathrm{m}$ the outer radius of the FET channel, respectively. The thermally grown $\mathrm{SiO}_{2}$ has a thickness $t_{o x} \approx 250 \mathrm{~nm}$. The p-MOSFET has been part of a test field processed together with a number of different silicon sensors, Fig. 5.1.

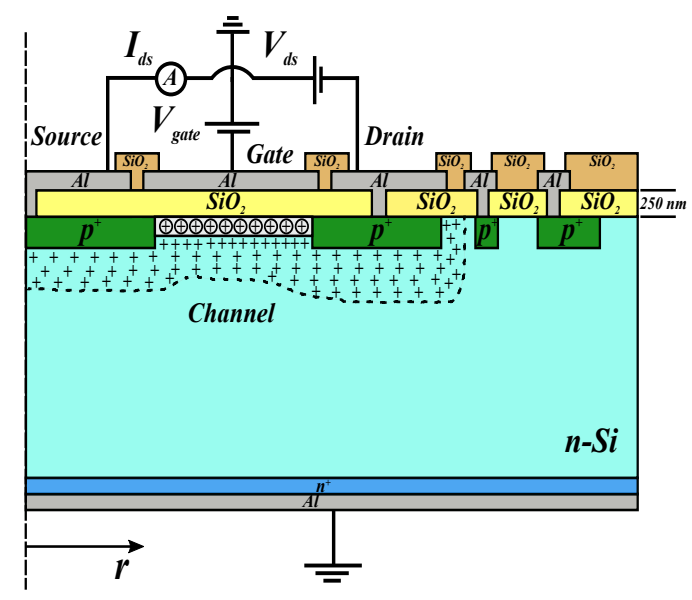

(a)

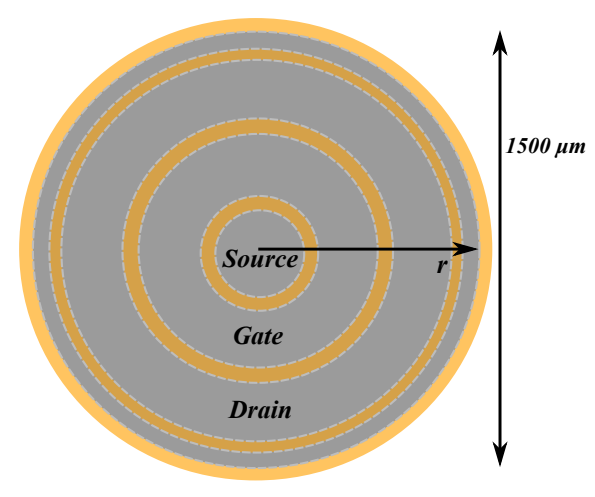

(b)

Figure 5.1: (a) Measurement setup, and cross section and (b) top view of the p-MOSFET $<111>$.

p-MOSFET characteristics The drain-source current, $I_{d s}$, of the p-MOSFET has been measured in the linear region at a drain-source voltage $V_{d s}=-50 \mathrm{mV}$. An example of a measured $I_{d s}\left(V_{g a t e}\right)$ curve is shown in Fig. 5.2 (a). The linear region of the p-MOSFET for $V_{d s}=-50 \mathrm{mV}$ is shown in Fig. 5.2 (b) where is plotted $I_{d s}$ as a function of $V_{d s}$ for different $V_{g a t e}$ values above the threshold voltage. For the p-MOSFET biased in inversion, where $V_{\text {gate }}<V_{\text {th }}$, the $I_{d s}\left(V_{\text {gate }}\right)$ measurements have been fitted by [32]

$$
I_{d s}=\mu C_{o x} \frac{W}{L} V_{d s}\left|V_{g a t e}-V_{t h}\right|
$$




$$
\mu=\frac{\mu_{0}}{1+\left(V_{\text {gate }}-V_{\text {th }}\right) / V_{1 / 2}} .
$$

The oxide capacitance per unit area is denoted by $C_{o x}$, the threshold voltage by $V_{t h}$, and the mobility of the holes, which are the charge carriers in the conducting channel of a p-MOSFET, by $\mu$. For the p-MOSFET, biased as shown in Fig. 5.1 (a), $V_{d s}$ and thus also $I_{d s}$ are negative. As $V_{g a t e}$ in inversion and $V_{t h}$ are also negative, $-I_{d s}$ versus $-V_{\text {gate }}$ is shown in Fig. 5.2 (a). The dependence of $\mu$ on the electric field transverse to the conducting channel, $E_{\text {field }}=\left(V_{\text {gate }}-V_{t h}\right) / t_{o x}$, is parameterized using Eq. 5.2. The hole mobility at $E_{\text {field }} \approx 0$ is $\mu_{0}$, and the value of $V_{\text {gate }}-V_{t h}$, at which the mobility has decreased by a factor 2 , is $V_{1 / 2}$. A positive $E_{\text {field }} \downarrow$ corresponds to an electric field pointing from the $\mathrm{SiO}_{2}$ into the $\mathrm{Si}$.

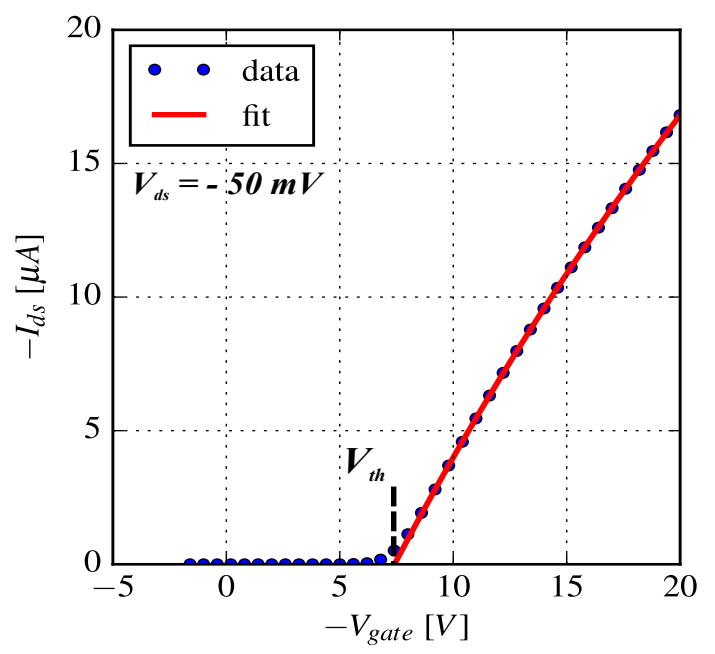

(a)

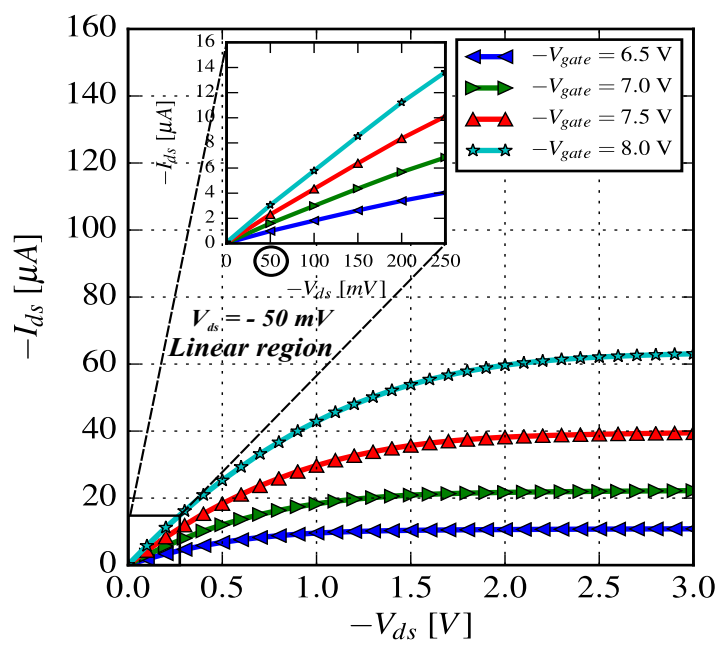

(b)

Figure 5.2: (a) Drain-source current, $I_{d s}$, as a function of gate voltage, $V_{g a t e}$, in the linear region, and the results of the fit used to determine the threshold voltage, $V_{t h}$, and the hole mobility of p-MOSFET, (b) Drainsource current, $I_{d s}$, as a function of drain-source voltage, $V_{d s}$, for different $V_{g a t e}$ values showing the linear region for $V_{d s}=-50 \mathrm{mV}$.

The free parameters of the fit are $V_{t h}, \mu_{0}$ and $V_{1 / 2}$ [122]. This parametrization provides a good description of $I_{d s}\left(V_{\text {gate }}\right)$, with deviations between the fit and the data of $<3 \%$ for $V_{\text {gate }} \lesssim \mathrm{V}_{\text {th }}-3 \mathrm{~V}$. The accurate evaluation of the $V_{t h}$ extraction, also has been checked with the square root conductance method proposed from Ghibaudo [123-126]. In the linear region of the MOSFET the transconductance $g_{m}$ is given by the Eq. 5.3.

$$
\begin{gathered}
g_{m}=\left.\frac{\partial I_{d s}}{\partial V_{g a t e}}\right|_{V_{d s}=c o n s t .}=\frac{\mu_{0}}{\left[1+\left(V_{\text {gate }}-V_{t h}\right) / V_{1 / 2}\right]^{2}} C_{o x} \frac{W}{L} V_{d s} \\
\frac{\left|I_{d s}\right|}{\sqrt{\left|g_{m}\right|}}=\frac{\frac{\mu_{0}}{1+\left(V_{\text {gate }}-V_{t h}\right) / V_{1 / 2}} C_{o x} \frac{W}{L}\left|V_{d s}\right|\left(\left|V_{\text {gate }}-V_{t h}\right|\right)}{\frac{\sqrt{\mu_{0} C_{o x} \frac{W}{L}\left|V_{d s}\right|}}{1+\left(V_{\text {gate }}-V_{t h}\right) / V_{1 / 2}}}=\sqrt{\mu_{0} C_{o x} \frac{W}{L}\left|V_{d s}\right|\left(\left|V_{\text {gate }}-V_{t h}\right|\right)}
\end{gathered}
$$


The calculation of the ratio $\left|I_{d s}\right| / \sqrt{\left|g_{m}\right|}$ results in Eq. 5.4. So it can be seen that the ratio $\left|I_{d s}\right| / \sqrt{\left|g_{m}\right|}$ does not depend on the parameter $V_{1 / 2}$. In Fig. 5.3 (b) is shown that the ratio $\left|I_{d s}\right| / \sqrt{\left|g_{m}\right|}$ has a linear dependence on $V_{\text {gate }}$. With a straight line fit the threshold voltage $V_{t h}$ and the mobility $\mu_{0}$ can be extrapolated. The linearity of the curve, Fig. 5.3 (a), as a function of $V_{\text {gate }}$, allows a cross check of the parameters obtained with the parametrization fit, Fig. 5.2 (a).

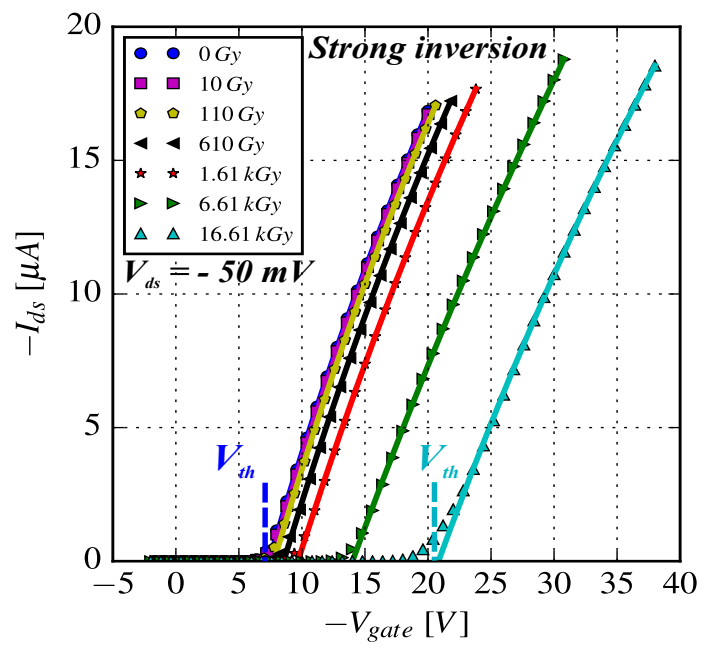

(a)

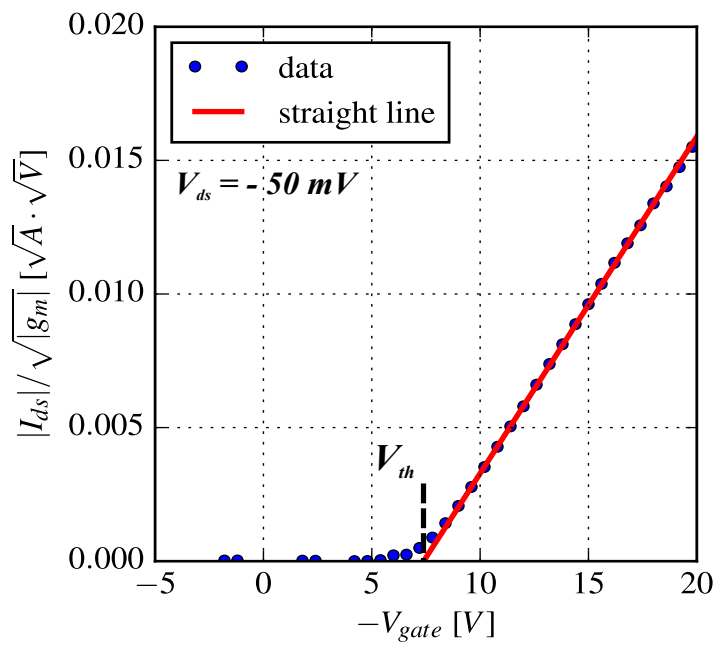

(b)

Figure 5.3: (a) $I_{d s}$ as a function of $V_{\text {gate }}$ in the linear region, and the results of the fits used to determine $V_{t h}$ and $\mu_{0}$ of p-MOSFET irradiated with $E_{\text {field }} \uparrow$ for the different X-ray dose values, (b) Ratio $\left|I_{d s}\right| / \sqrt{\left|g_{m}\right|}$ as a function of $V_{\text {gate }}$ and straight line fit before X-ray irradiation.

The difference in the parameters $\mu_{0}$ and $V_{t h}$ which were extracted with the two different methods is less than $1.5 \%$, as presented in the Table 5.1.

Table 5.1: Comparison of the two different extraction methods of the threshold voltage $\mathrm{V}_{t h}$.

\begin{tabular}{lll}
\hline Extraction methods & \multicolumn{2}{c}{ Parameters } \\
\cline { 2 - 3 } & $\mu_{0}\left[\frac{\mathrm{cm}^{2}}{V \cdot s}\right]$ & $V_{t h}[\mathrm{~V}]$ \\
\hline Parametrization fit & 257.1 & 7.41 \\
Square root conductance & 253.8 & 7.39 \\
\hline Difference [\%] & 1.3 & 0.3 \\
\hline
\end{tabular}

From $V_{t h}$, the effective oxide charge density, $N_{o x}^{e f f}$, is obtained by Eq. 5.5 with (-) for n-bulk Si

$$
N_{o x}^{e f f}=\frac{C_{o x}\left(-V_{t h}+2 \phi_{b}+\phi_{m s} \mp \frac{\left.\sqrt{2 \varepsilon_{s i} \varepsilon_{0} q_{0} N_{d}\left|\phi_{b}\right|}\right)}{C_{o x}},\right.}{q_{0}},
$$

where $\phi_{b}=-0.1147 \mathrm{~V}$ has been used for the potential difference between the midgap potential and the Fermi level in the $\mathrm{Si}$, and $\phi_{m s}=-0.4235 \mathrm{~V}$ for the Al-Si work function difference. From $V_{1 / 2}$, the surface roughness amplitude, $\Delta$, which corresponds to the width of the region in the Si in which the hole mobility is 
lower than the bulk mobility of $\approx 450 \mathrm{~cm}^{2} /(\mathrm{V} \cdot \mathrm{s})$, can be calculated using

$$
\Delta=\frac{\varepsilon_{S i} \varepsilon_{0}}{\eta C_{o x} q_{0}\left|V_{1 / 2}\right| / k T}
$$

where $\varepsilon_{S i}$ is the dielectric constant of $\mathrm{Si}, \varepsilon_{0}$ the dielectric permittivity of the vacuum, $q_{0}$ the elementary charge, $k$ the Boltzmann constant, $T$ the absolute temperature, and $\eta$ a weighting factor, which is equal to $1 / 3$ for holes $[47-49,127]$.

\subsubsection{Analysis method}

Fig. 5.4 shows the measurement cycle adopted for the study of the X-ray dose and electric field dependence of surface radiation damage. On the top is shown, as a function of the measurement time, $t$, the applied gate voltage $V_{\text {gate }}(t)$, and on the bottom, the measured drain-source current $I_{d s}(t)$.

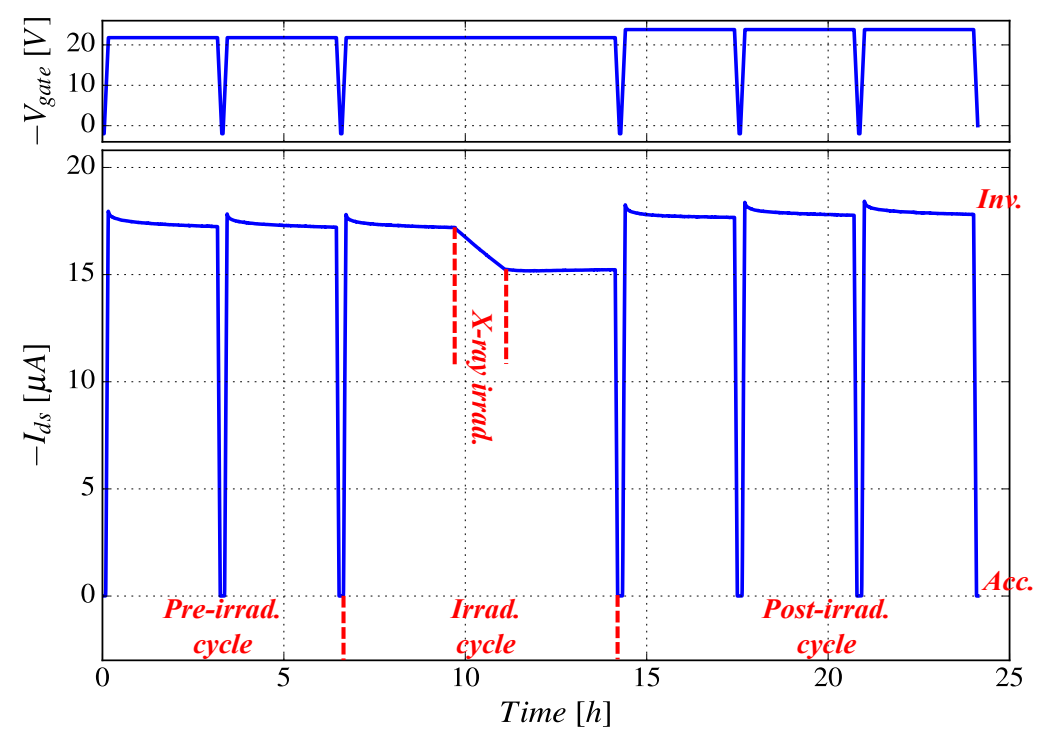

Figure 5.4: Measurement cycle: the applied gate voltage, $V_{\text {gate }}(t)$, is shown on the top, and the measured drain-source current, $I_{d s}(t)$, on the bottom of p-MOSFET irradiated at $1 \mathrm{kGy}$ X-ray dose with $E_{\text {field }} \uparrow$.

In the pre-irradiation cycle, $V_{\text {gate }}$ is changed from $+2 \mathrm{~V}$ to $V_{i n v}$, kept at $V_{i n v}$ for 3 hours, and then changed back to $+2 \mathrm{~V}$. After 2 minutes at $V_{\text {gate }}=+2 \mathrm{~V}$, this sequence is repeated. Two sequences are used, in order to check the reproducibility of the measurement results. In the irradiation cycle, $V_{\text {gate }}$ is again changed to $V_{i n v}$, left at this voltage for 3 hours, followed by the X-ray irradiation and another 3 hours at $V_{i n v}$, after which $V_{\text {gate }}$ is changed back to $+2 \mathrm{~V}$. The irradiation cycle is followed by the post-irradiation cycle. The value of $V_{i n v}$ for the pre-irradiation and the irradiation cycle is chosen so that the electric field in the oxide $E_{\text {field }}^{\text {pre irr }} \approx-500 \mathrm{kV} / \mathrm{cm}$. The build-up of positive oxide charges during the irradiation, results in a change of $V_{t h}$, and thus of $E_{\text {field }}$. In order to apply approximately the same $E_{\text {field }}$ value in the post-irradiation cycle, $V_{i n v}$ is changed and the corresponding electric field is called $E_{\text {field }}^{\text {post }}$. .

For the irradiation an X-ray tube with a W anode, operated at $35 \mathrm{kV}$, has been used. The dose rate in the $\mathrm{SiO}_{2}$ has been calibrated to an accuracy of about $10 \%$ using the photocurrent produced by the X-rays in a silicon pad diode. The p-MOSFET has been irradiated to a total ionizing dose of $16.61 \mathrm{kGy}\left(\mathrm{SiO}_{2}\right)$ in steps of 10 , 
$100,500,1 \mathrm{k}$ and $5 \mathrm{kGy}$ with a dose rate of $0.2 \mathrm{~Gy} / \mathrm{s}$, and a last step of $10 \mathrm{kGy}$ with a dose rate of $0.3 \mathrm{~Gy} / \mathrm{s}$. During the measurements and the storage, the p-MOSFET was kept at a temperature of about $23^{\circ} \mathrm{C}$.

From the measured $I_{d s}\left(V_{\text {gate }}\right)$ curve, for which $V_{\text {gate }}$ was changed from $+2 \mathrm{~V}$ to $V_{i n v}$, the values of $V_{t h}, \mu_{0}$ and $V_{1 / 2}$ of the forward calibration are obtained using Eq. 5.1, and the reverse calibration when changing $V_{\text {gate }}$ from $V_{i n v}$ to $+2 \mathrm{~V}$. Using Eq. 5.5, is calculated from the $V_{t h}$ values $N_{o x, f o r w}^{e f f}$ and $N_{o x, r e v}^{e f f}$. In order to determine $N_{o x}^{e f f}(t)$ when $V_{g a t e}=V_{i n v}$, Eq. 5.1 is inverted to calculate $V_{t h}(t)$ from the measured $I_{d s}(t)$, Eq. 5.7.

$$
\begin{gathered}
V_{t h}(t)=\frac{I_{d s}(t)}{\frac{I_{d s}(t)}{V_{1 / 2}}-\mu_{0} C_{o x} \frac{W}{L} V_{d s}}+V_{g a t e} \\
V_{t h}^{d u r}(t)=V_{t h}^{b e f}(t)\left(1-\frac{t}{t_{d u r}}\right)+V_{t h}^{a f t e r}(t) \frac{t}{t_{d u r}}
\end{gathered}
$$

For the part before irradiation the extracted parameters $\mu_{0}^{\text {pre }}$ and $V_{1 / 2}^{\text {pre }}$ from the $I_{d s}\left(V_{\text {gate }}\right)$ calibration curve measured in the reverse direction have been used. For the part after irradiation have been used the extracted parameters $\mu_{0}^{\text {post }}$ and $V_{1 / 2}^{\text {post }}$ from the calibration curve measured in the reverse direction. For the $1 \mathrm{kGy}$ irradiation the calibration curves before and after are shown in Fig. 5.5 (a).

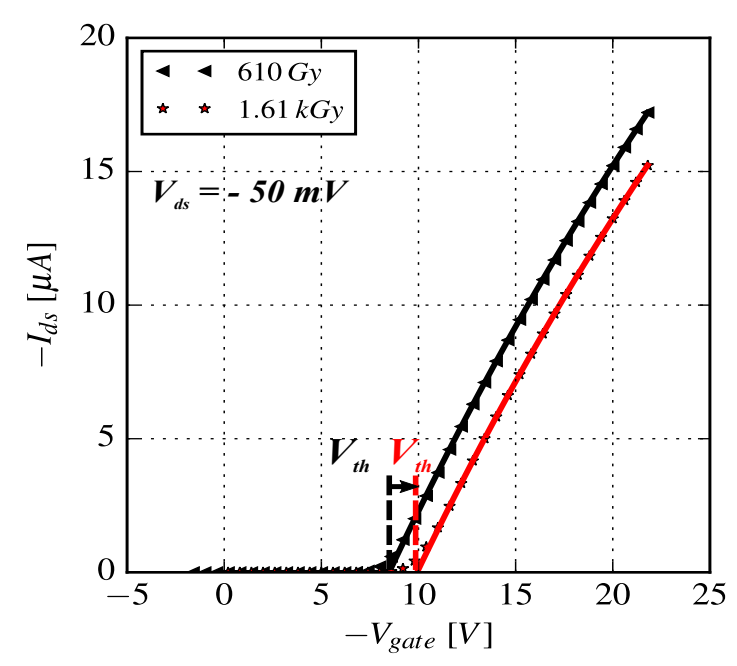

(a)

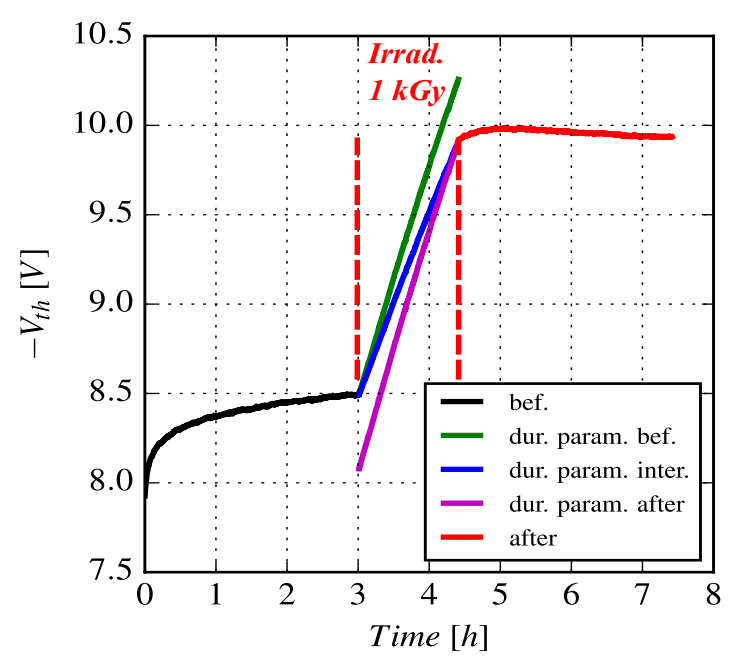

(b)

Figure 5.5: (a) Drain-source current, $I_{d s}$, as a function of gate voltage, $V_{\text {gate }}$, in the linear region before and after $1 \mathrm{kGy}$ irradiation, and (b) Threshold voltage, $V_{t h}(t)$, before, during and after $1 \mathrm{kGy}$ irradiation showing the linear interpolation method.

For the part during irradiation, the linear interpolation of the $V_{t h}$ change of the regions before and after irradiation has been assumed, in order to describe $V_{t h}(t)$. In this way is measured the $N_{o x}^{e f f}(t)$ also during the irradiation, as the hole mobility changes. Eq. 5.8 describes the threshold voltage as a function of time during irradiation $V_{t h}^{\text {dur }}(t)$, where $V_{t h}^{\text {bef }}(t), V_{t h}^{a f t e r}(t)$ and $t_{d u r}$ are the threshold voltage as a function of time before, after the irradiation and $t_{d u r}$ the time duration of the irradiation, as shown in Fig. 5.5 (b) for the $1 \mathrm{kGy}$ irradiation. Fig. 5.3 (a) shows the $I_{d s}\left(V_{\text {gate }}\right)$ calibration curves together with the fits for the different irradiations. It is found that the values of $V_{t h}$ change from $\approx-7.5 \mathrm{~V}$ to $\approx-21.0 \mathrm{~V}$ due to the increase of 
positive oxide charges. The observed decrease of the slope of the $I_{d s}\left(V_{\text {gate }}\right)$ curves with dose is the result of the reduction of the hole mobility. The deviation of the $I_{d s}\left(V_{\text {gate }}\right)$ curves from linearity for large negative $V_{\text {gate }}$ values is due to the decrease of the hole mobility with increasing $\left|E_{\text {field }}\right|$ at the $\mathrm{Si}_{-} \mathrm{SiO}_{2}$ interface. Next is presented the time dependence of the drain-source current, $I_{d s}(t)$, which is shown in Figs. 5.4 and 5.6 for the $1 \mathrm{kGy}$ irradiation.

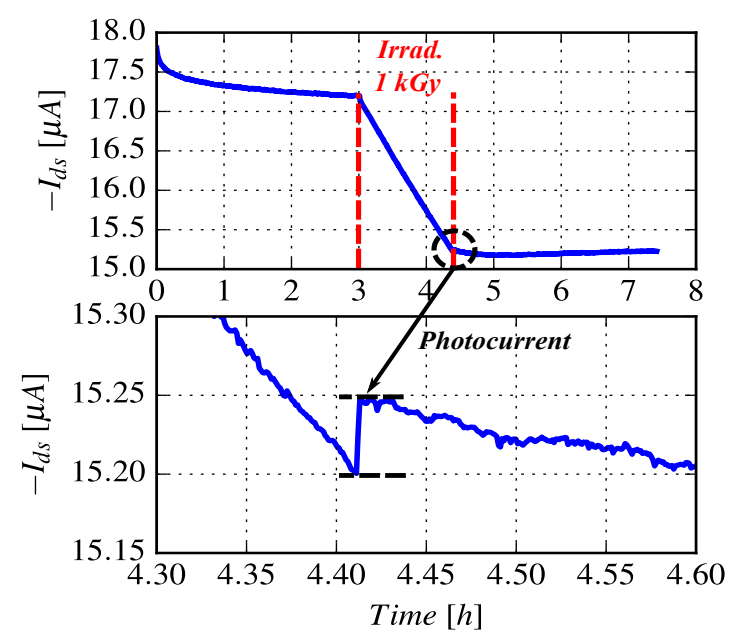

(a)

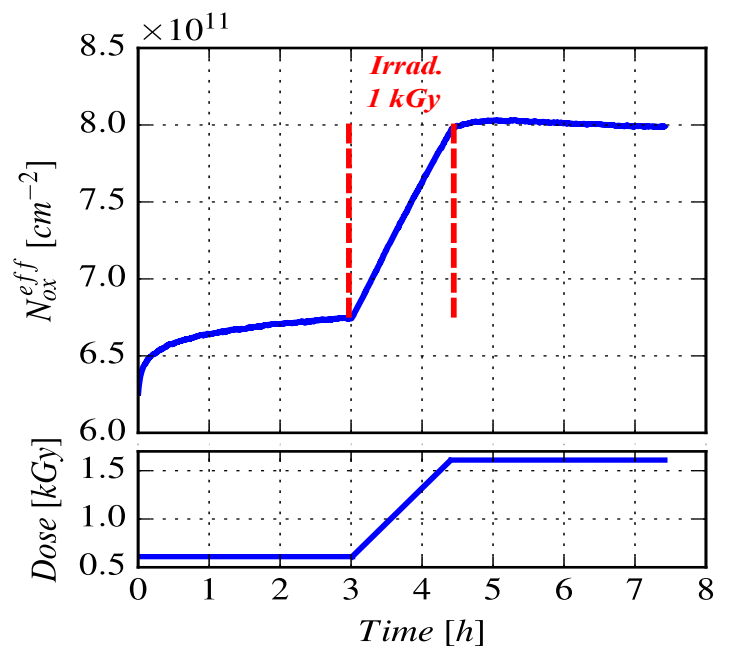

(b)

Figure 5.6: (a) Drain-source current, $I_{d s}(t)$, and (b) Effective oxide charge density, $N_{o x}^{\text {eff }}(t)$, of the pMOSFET before, during and after the $1 \mathrm{kGy}$ irradiation. At $t=3 \mathrm{~h}$ the X-ray source has been switched on, which results in an additional photocurrent. At $t=4.41 \mathrm{~h}$ the X-ray source has been switched off, resulting in the step of $I_{d s}$ due to the photocurrent seen at the bottom of (a).

After every forward calibration, $I_{d s}$ decreases with time at the constant gate voltage $V_{g a t e}=V_{i n v}$. The time constant is of the order of 30 minutes. A decrease of $I_{d s}$ corresponds to an increase of $N_{o x}^{e f f}$. For $V_{g a t e}=V_{\text {inv }}$ the MOSFET is in strong inversion: at the $\mathrm{Si}_{-} \mathrm{SiO}_{2}$ interface a hole inversion layer has formed, and the electric field $E_{\text {field }}=\left(V_{i n v}-V_{t h}\right) / t_{o x}$ points $\uparrow$ from the $\mathrm{Si}$ into the $\mathrm{SiO}_{2}$, Fig. 5.7. As a result, border traps are charged up positively [128-134]. The increase of the density of charged border traps is defined as $\Delta N_{o x, c h}=N_{o x}^{e f f}\left(t_{0}+3 \mathrm{~h}\right)-N_{o x}^{e f f}\left(t_{0}\right)$, where $t_{0}$ is the time at which $V_{g a t e}=V_{i n v}$ was reached for the forward calibration. Fig. 5.4 shows that after every forward calibration approximately the same decrease of $I_{d s}$ is observed.

As seen in Fig. 5.4, the value of $I_{d s}$ at the start of the reverse calibration is lower than the value at the end of the forward calibration. This corresponds to a reduction of $N_{o x}^{e f f}$. For $V_{g a t e} \gg V_{t h}$, the p-MOSFET is in accumulation: at the $\mathrm{Si}_{-} \mathrm{SiO}_{2}$ interface an electron accumulation layer has formed, and the electric field points $\downarrow$ from the $\mathrm{SiO}_{2}$ into the $\mathrm{Si}$. As a result, border traps are discharged. The decrease of the density of charged border traps is defined as $\Delta N_{o x, \text { disch }}=N_{o x, \text { forw }}^{e f f}-N_{o x, \text { rev }}^{e \text {. }}$.

Fig. 5.6 shows the irradiation cycle for the $1 \mathrm{kGy}$ irradiation. When the X-ray source is switched on at $t=3 \mathrm{~h}, I_{d s}$ decreases instantaneously due to the photocurrent generated by the X-rays. As seen at the bottom of Fig. 5.6 (a), the opposite, an instantaneous increase of $I_{d s}$ is observed, when the X-ray source is switched off at $t=4.41 \mathrm{~h}$. During the X-ray irradiation, $I_{d s}$ decreases approximately linearly with time, due to the 
build-up of radiation-induced positive charges, shown in Fig. 5.6 (b). After switching off the X-ray source, a further increase of $N_{o x}^{e f f}$ is observed, which is again due to the charging up of border traps. The effective oxide charge density after irradiation to a dose $D$ is defined as $N_{o x, \text { dose }}^{\text {eff }}(D)=N_{o x}^{\text {eff }}\left(t_{1}+3 \mathrm{~h}\right)$, where $t_{1}$ is the time of the end of the irradiation.

\subsubsection{Effective oxide charge density $N_{o x}^{e f f}$}

Table 5.2: Irradiation conditions and extracted values of the surface radiation parameters as a function of X-ray dose of the p-MOSFET $<111>$ irradiated with $E_{\text {field }} \uparrow$.

\begin{tabular}{|c|c|c|c|c|c|c|c|c|c|c|c|c|}
\hline $\begin{array}{c}\text { Dose } \\
{[\mathrm{Gy}]}\end{array}$ & $\begin{array}{l}V_{a c c} \\
{[\mathrm{~V}]}\end{array}$ & $\begin{array}{l}V_{i n v}^{i r r} \\
{[\mathrm{~V}]}\end{array}$ & $\begin{array}{c}E_{\text {field }}^{a c c} \\
{[\mathrm{kV} / \mathrm{cm}]}\end{array}$ & $\begin{array}{c}E_{\text {field }}^{\text {pre irr }} \\
{[\mathrm{kV} / \mathrm{cm}]}\end{array}$ & $\begin{array}{c}E_{\text {field }}^{\text {irr }} \\
{[\mathrm{kV} / \mathrm{cm}]}\end{array}$ & $\begin{array}{l}E_{\text {field }}^{\text {post irr }} \\
{[\mathrm{kV} / \mathrm{cm}]}\end{array}$ & $\begin{array}{l}N_{o x, \text { dose }}^{\text {eff }} \\
{\left[\mathrm{cm}^{-2}\right]}\end{array}$ & $\begin{array}{l}\Delta N_{o x, c h} \\
{\left[\mathrm{~cm}^{-2}\right]}\end{array}$ & $\begin{array}{c}\Delta N_{\text {ox }, \text { disch }} \\
{\left[\mathrm{cm}^{-2}\right]}\end{array}$ & $\begin{array}{c}\mu_{0} \\
{\left[\mathrm{~cm}^{2} /(\mathrm{Vs})\right]}\end{array}$ & $\begin{array}{l}V_{1 / 2} \\
{[\mathrm{~V}]}\end{array}$ & $\begin{array}{c}\Delta \\
{[\mathrm{nm}]}\end{array}$ \\
\hline 0 & 2.0 & -20.0 & 380 & -500 & -500 & -500 & $52.3 \cdot 10^{10}$ & $5.3 \cdot 10^{10}$ & $5.8 \cdot 10^{10}$ & 257 & -61 & 0.95 \\
\hline 10 & 2.0 & -20.0 & 380 & -500 & -500 & -500 & $59.3 \cdot 10^{10}$ & $4.6 \cdot 10^{10}$ & $5.6 \cdot 10^{10}$ & 259 & -60 & 0.97 \\
\hline 110 & 2.0 & -20.0 & 390 & -500 & -490 & -510 & $61.7 \cdot 10^{10}$ & $4.7 \cdot 10^{10}$ & $5.7 \cdot 10^{10}$ & 257 & -61 & 0.95 \\
\hline 610 & 2.0 & -20.6 & 420 & -510 & -500 & -530 & $69.0 \cdot 10^{10}$ & $4.6 \cdot 10^{10}$ & $6.2 \cdot 10^{10}$ & 250 & -66 & 0.88 \\
\hline 1610 & 2.0 & -21.8 & 470 & -530 & -500 & -570 & $79.8 \cdot 10^{10}$ & $5.0 \cdot 10^{10}$ & $7.1 \cdot 10^{10}$ & 237 & -78 & 0.75 \\
\hline 6610 & 2.0 & -23.8 & 640 & -570 & -460 & -680 & $124.5 \cdot 10^{10}$ & $7.6 \cdot 10^{10}$ & $11.5 \cdot 10^{10}$ & 207 & -111 & 0.53 \\
\hline 16610 & 2.0 & -29.0 & 910 & -640 & -440 & -730 & $189.8 \cdot 10^{10}$ & $8.9 \cdot 10^{10}$ & $18.8 \cdot 10^{10}$ & 200 & -103 & 0.57 \\
\hline
\end{tabular}

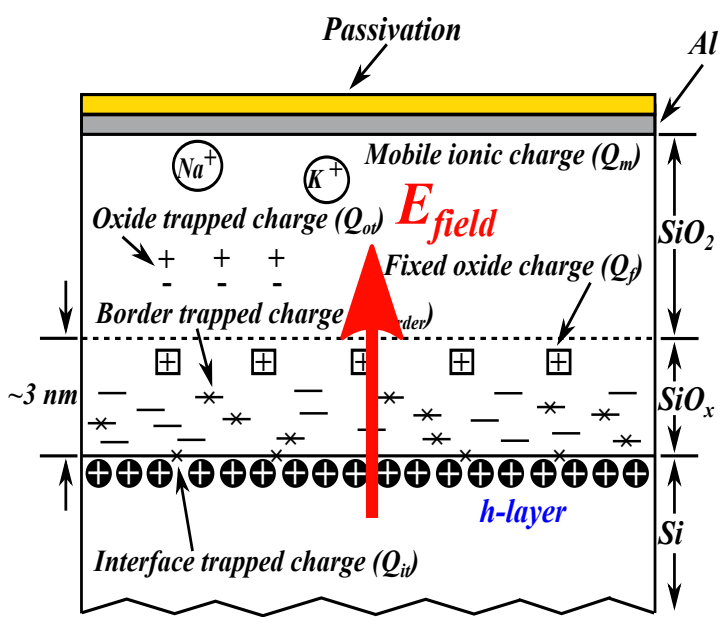

Figure 5.7: Nomenclature of oxide charges, hole inversion layer and $E_{\text {field }} \uparrow$ direction during irradiation.

Table 5.2 presents for the individual irradiation steps the integrated dose values, the values of $V_{\text {gate }}$ in accumulation $V_{a c c}$, of $V_{i n v}^{i r r}$ applied to the gate for the pre-irradiation and irradiation cycles, the electric field in accumulation $E_{\text {field }}^{\text {acc }}$, the electric field for the pre- and post-irradiation cycles, $E_{\text {field }}^{\text {pre irr }}$ and $E_{\text {field }}^{\text {post irr }}$, the average electric field during the irradiation, $E_{\text {field }}^{i r}$, and the results for the effective oxide charge density and the hole mobility. Fig. 5.8 (a) shows for the individual irradiation steps $N_{o x}^{\text {eff }}(t)$ during the irradiation cycles, and the eighth column of Table 5.2 the values of $N_{o x, \text { dose }}^{e f f}(D)$, the effective oxide charge density determined at the end of each irradiation cycle. Before irradiation, $N_{o x, \text { dose }}^{e f f}(0)=5.2 \cdot 10^{11} \mathrm{~cm}^{-2}$, which is in the range expected for $\mathrm{Si}$ with $<111>$ orientation. For the maximum dose of $16.61 \mathrm{kGy}\left(\mathrm{SiO}_{2}\right)$, $N_{o x, \text { dose }}^{\text {eff }}(16.61 \mathrm{kGy})=1.9 \cdot 10^{12} \mathrm{~cm}^{-2}$. From the values shown in the Table 5.2 as well as from Fig. 5.8 (a), 
one sees that the derivative $\mathrm{d} N_{o x, \text { dose }}^{e f f}(D) / \mathrm{d} D$ decreases with dose, where it has to be taken into account that for the $10 \mathrm{kGy}$ irradiation step the dose rate has been increased by a factor of 1.5. Fig. 5.8 (b) shows $E_{\text {field }}(t)$ during the irradiation cycles for the individual steps. The irradiations performed at constant $V_{\text {gate }}$, at the $V_{i n v}^{i r r}$ values of the third column of Table 5.2. So the $\left|E_{\text {field }}\right|$ was decreasing during irradiation due to the increase of $N_{o x}^{e f f}$. For the higher irradiation step of $10 \mathrm{kGy}$, the decrease was $\approx 60 \%$. The irradiation with the electric field pointing $\uparrow$ from the $\mathrm{Si}$ into the $\mathrm{SiO}_{2}$, Fig. 5.7, minimizes the build-up of positive charges in the oxide, and much larger effects are expected for the opposite field direction.

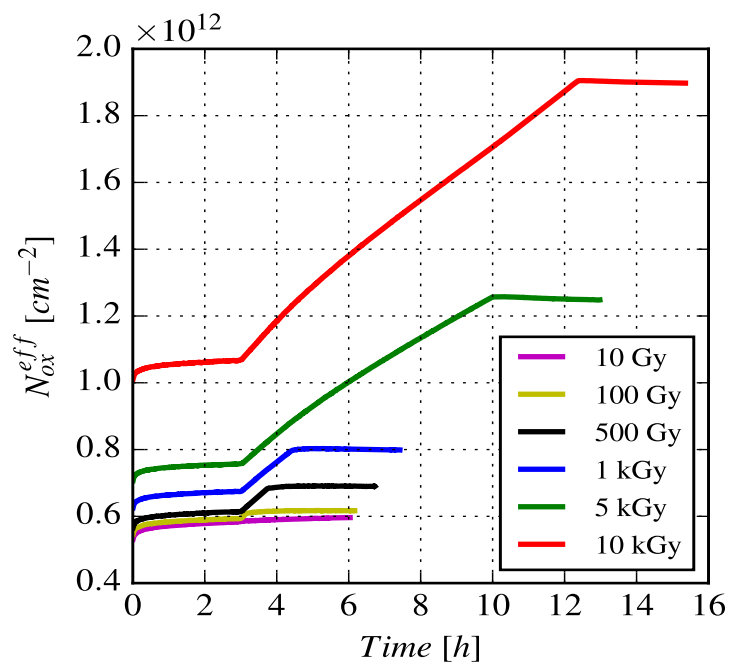

(a)

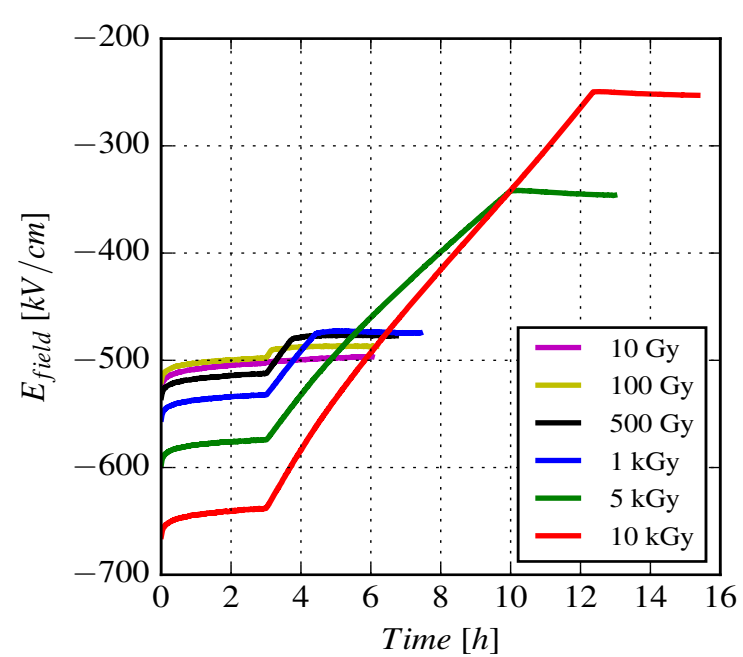

(b)

Figure 5.8: (a) Effective oxide charge density, $N_{o x}^{e f f}(t)$ and (b) Electric field $E_{\text {field }}$ as a function of time for the individual irradiation cycles of p-MOSFET irradiated with $E_{\text {field }} \uparrow$.

Border traps and hole mobility The effects of border traps, near-interfacial oxide traps that can communicate with the underline Si over a wide range of time scales, on the p-MOSFET long-term response are presented. The ninth column of Table 5.2 shows the X-ray dose dependence of $\Delta N_{o x, c h}(D)$, the charging up of the border traps during the three hours after irradiation, and the tenth column $\Delta N_{o x, \text { disch }}(D)$, the discharging of the border traps, when changing $V_{\text {gate }}$ from inversion to accumulation, remaining 2 minutes in accumulation and then biasing back to inversion. A significant charging up and discharging of border traps, at the level of $5-10 \%$ of $N_{o x}^{e f f}$, is observed. Fig. 5.9 (a) shows the charging up of border traps at constant $V_{g a t e}$ for three hours in inversion, $N_{o x}^{e f f}$ increases and the discharging, $N_{o x}^{\text {eff }}$, decreases after switching from inversion to accumulation. In addition, Fig. 5.9 (b) shows that during charging up a hole layer is present at the interface and the $E_{\text {field }}^{\text {post irr }}$ points $\uparrow$ from the $\mathrm{Si}$ to the $\mathrm{SiO}_{2}$ and during discharging an electron layer is present with $E_{\text {field }}^{a c c}$ pointing $\downarrow$ from the $\mathrm{SiO}_{2}$ to the $\mathrm{Si}$. For the highest dose values, where also the values of $\left|E_{\text {field }}^{\text {postirr }}\right|$ and $E_{\text {field }}^{\text {acc }}$ have been the highest, the biggest values of $\Delta N_{o x, c h}(D)$ and of $\Delta N_{o x, \text { disch }}(D)$ are observed, shown in Fig. 5.10.

As discussed in the previous section, the $I_{d s}\left(V_{\text {gate }}\right)$ calibrations allow to determine the dependence of the hole mobility on $V_{\text {gate }}$ for the different dose values. For the parametrization of the mobility, Eq. 5.2, is used. The results for $\mu_{0}$ and $V_{1 / 2}$ for the reverse calibration curves following the irradiations, are shown in Table 5.2 


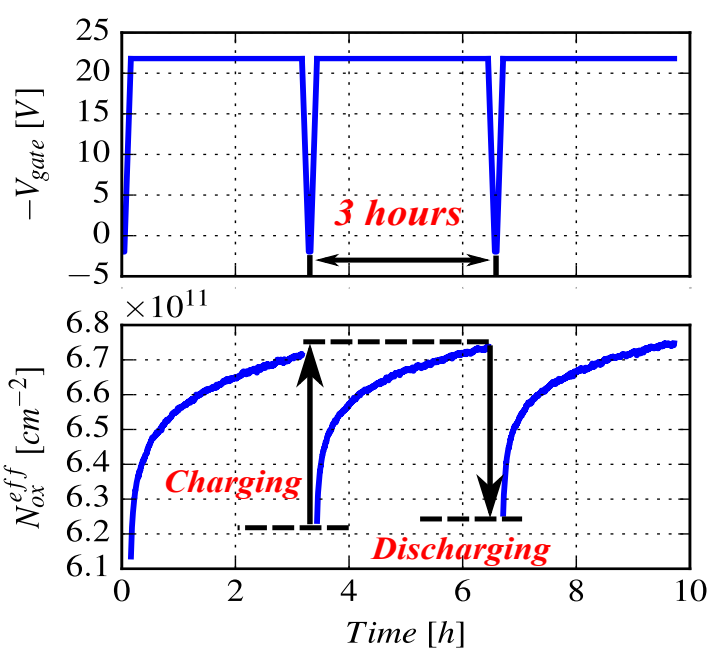

(a)

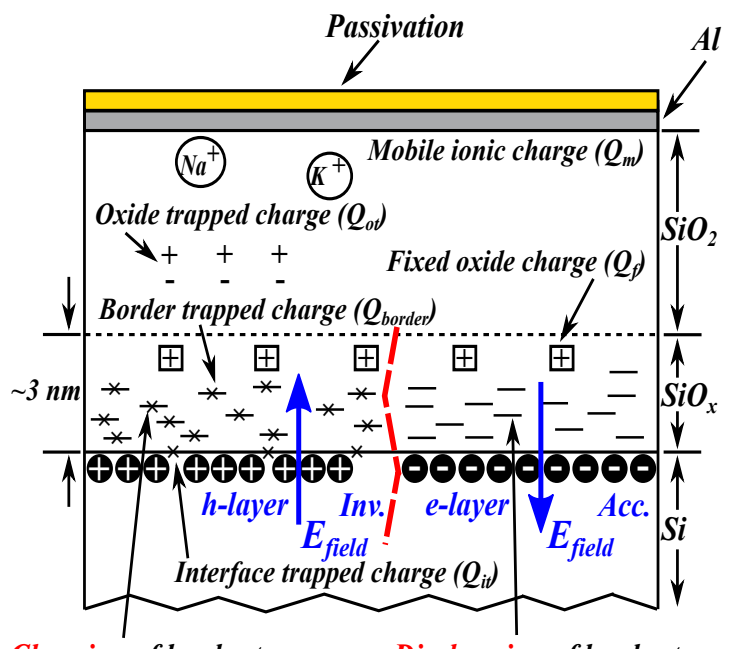

Discharging of border traps

(b)

Figure 5.9: (a) Gate voltage $V_{\text {gate }}$ and $N_{o x}^{e f f}$ as a function of time during the $3 \mathrm{~h}$ biasing after $1 \mathrm{kGy}$ irradiation, and (b) Nomenclature of oxide charges, charge layer at the interface and the $E_{\text {field }}$ direction during charging and discharging of border traps.

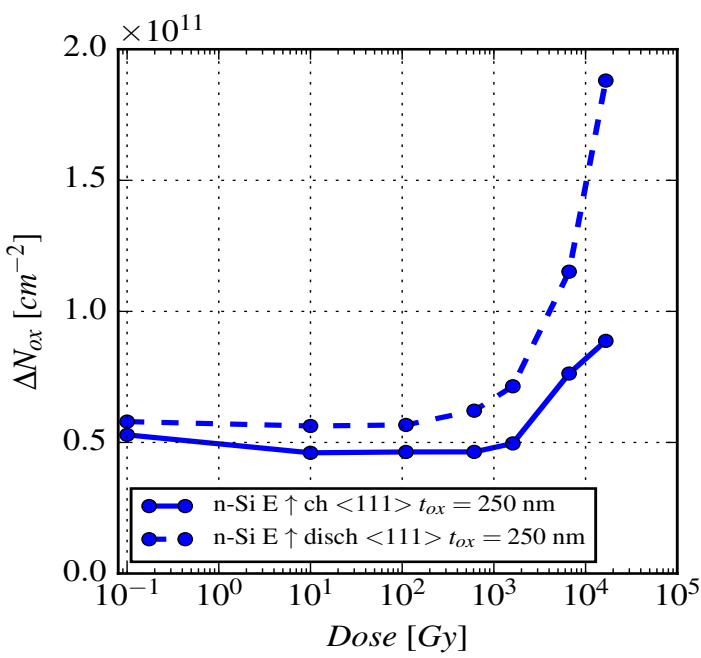

(a)

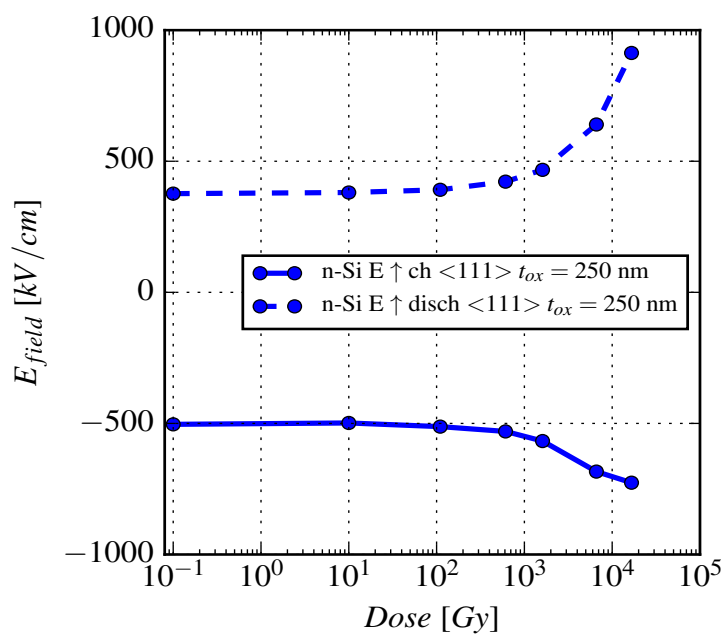

(b)

Figure 5.10: (a) $\Delta N_{o x}$ of charging and discharging of border traps, and (b) $E_{\text {field }}$ values during charging and discharging of border traps as a function of the accumulated X-ray dose of p-MOSFET irradiated with $E_{\text {field }}$ 


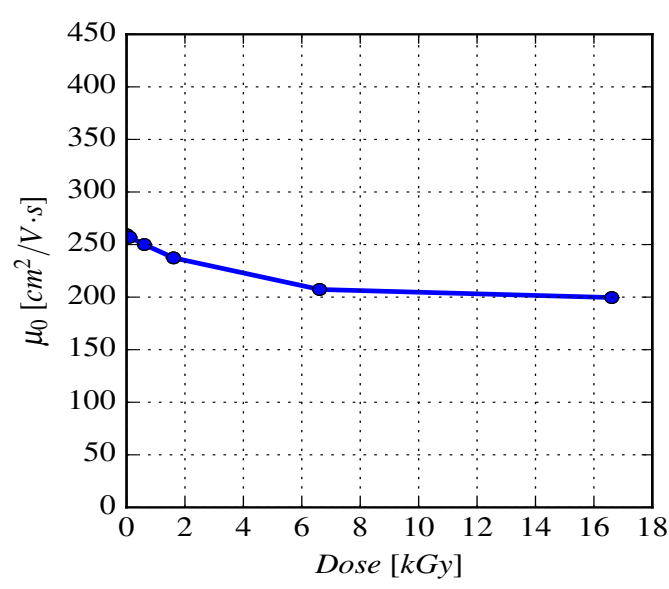

(a)

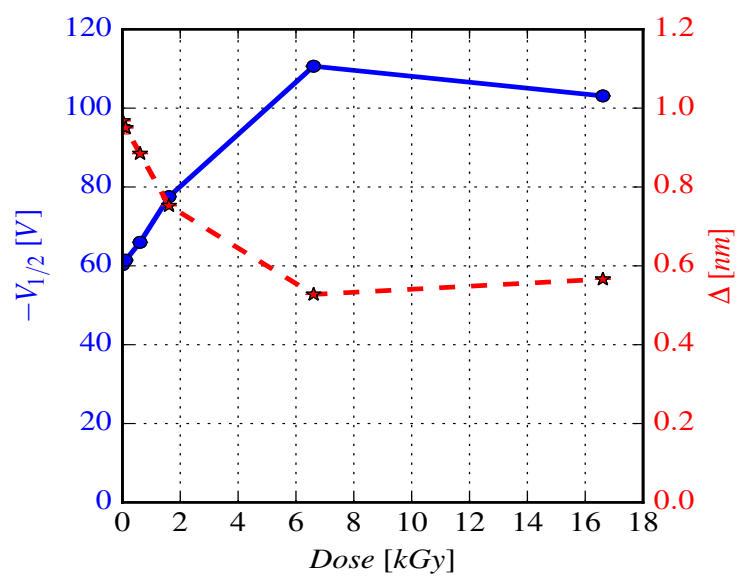

(b)

Figure 5.11: (a) Hole mobility, $\mu_{0}$, and (b) $V_{1 / 2}$ and $\Delta$ as a function of the accumulated X-ray dose of p-MOSFET irradiated with $E_{\text {field }} \uparrow$.

and in Fig. 5.11. For the non-irradiated p-MOSFET, a hole mobility at $E_{\text {field }} \approx 0$ of $\mu_{0}=257 \mathrm{~cm}^{2} /(\mathrm{V} \cdot \mathrm{s})$ is extracted, which is significantly smaller than the bulk mobility of $\approx 450 \mathrm{~cm}^{2} /(\mathrm{V} \cdot \mathrm{s})$. As expected, $\mu_{0}$ decreases with increasing dose. The value of $V_{1 / 2}$ changes with dose from $-61 \mathrm{~V}$ to about $-103 \mathrm{~V}$. Also in Fig. 5.11 (b) is presented the dose dependence of the surface roughness amplitude, $\Delta$, which is defined by Eq. 5.6. The surface radiation damage of $\mathrm{SiO}_{2}$ grown on high-ohmic $\mathrm{Si}$, which is used for the fabrication of silicon sensors, has been studied. Using a circular p-MOSFET, a method has been established to measure the effective oxide charge density in the $\mathrm{SiO}_{2}$ as a function of ionizing dose and electric field. The method was applied to a p-MOSFET fabricated by Canberra on Si with $<111>$ orientation and an oxide thickness of $250 \mathrm{~nm}$ for dose values of up to $\approx 17 \mathrm{kGy}$ and an electric field in the $\mathrm{SiO}_{2}$ of about $500 \mathrm{kV} / \mathrm{cm}$ pointing $\uparrow$ from the $\mathrm{Si}$ into the $\mathrm{SiO}_{2}$. The effective oxide charge density has been found to increase from about $5 \cdot 10^{11} \mathrm{~cm}^{-2}$ to close to $2 \cdot 10^{12} \mathrm{~cm}^{-2}$. By cycling the gate voltage between inversion and accumulation conditions, the charging and discharging of border traps has been observed, and the corresponding changes in effective oxide charge densities have been determined. In addition, the dependence of the hole mobility close to the $\mathrm{Si}_{-} \mathrm{SiO}_{2}$ as a function of electric field and dose has been extracted from the measurements. A significant reduction of the hole mobility with dose is observed. The work presented above was the first case towards the determination of the effective oxide charge density as a function of ionizing dose and electric field in the $\mathrm{SiO}_{2}$. Measurements with p- and n-MOSFET, where the situation is opposite and much bigger effects are expected, are presented in next sections. The aim of the investigation is to provide data on the dependence of the effective oxide charge density as a function of the electric field and the ionizing dose in the $\mathrm{SiO}_{2}$, in order to improve TCAD simulations of segmented silicon sensors, in which so far a position independent effective oxide charge density has been assumed [4]. In addition, the dependence of the mobility of electrons and holes close to $\mathrm{Si}-\mathrm{SiO}_{2}$ interface as a function of dose and electric field will be provided. 


\subsubsection{The p-MOSFET irradiated with $E_{\text {field }}$ pointing from the $\mathrm{SiO}_{2}$ into the $\mathrm{Si}$}

Similar to the methodology presented in the previous section, irradiations on a p-MOSFET with the $E_{\text {field }}$ pointing $\downarrow$ from the $\mathrm{SiO}_{2}$ into the $\mathrm{Si}$, have been performed. A circular p-MOSFET with the same dimensions as in Fig. 5.1, fabricated by Canberra on $<111>$ n-type Float-Zone (FZ) Si with the same process and bulk doping of $N_{d}=6.2 \cdot 10^{11} \mathrm{~cm}^{-3}$, has been used. The test structure biased in accumulation at a field in the $\mathrm{SiO}_{2}$ of about $500 \mathrm{kV} / \mathrm{cm}$ during irradiation and irradiated by X-rays up to a dose of about $17 \mathrm{kGy}\left(\mathrm{SiO}_{2}\right)$ in different irradiation steps. Before and after each irradiation, the gate voltage has been cycled from inversion to accumulation conditions and back and the threshold voltage and the hole mobility at the $\mathrm{Si}_{-} \mathrm{SiO}_{2}$ interface determined.

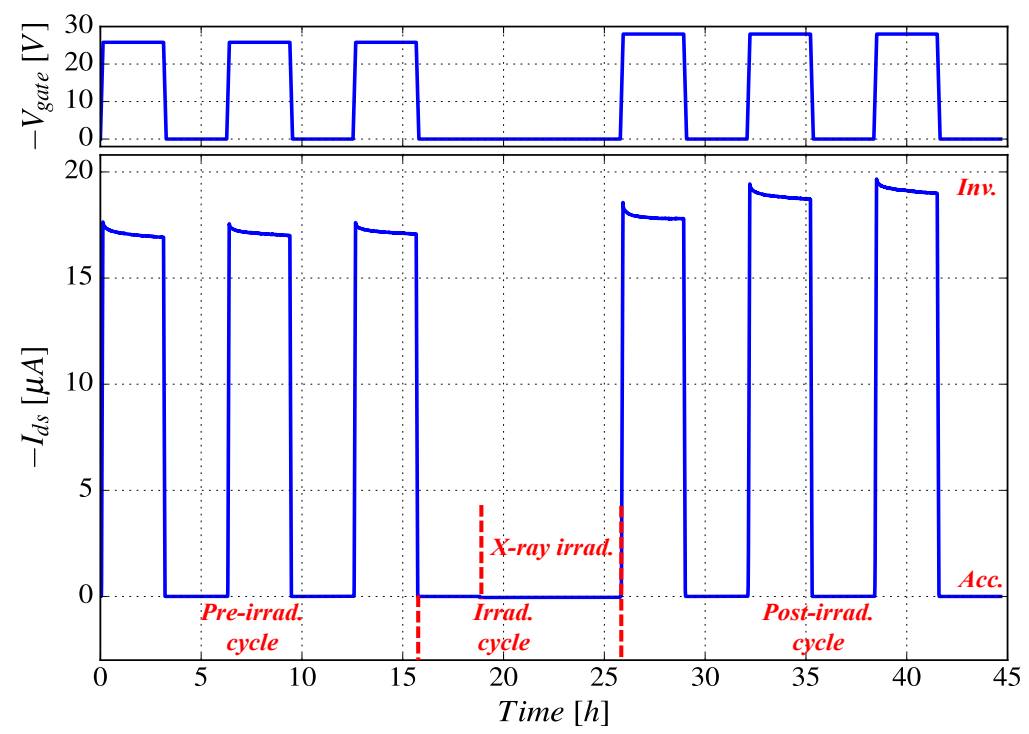

Figure 5.12: Measurement cycle: the applied gate voltage, $V_{\text {gate }}(t)$, is shown on the top, and the measured drain-source current, $I_{d s}(t)$, on the bottom of p-MOSFET irradiated at $5 \mathrm{kGy}$ X-ray dose with $E_{\text {field }} \downarrow$.

Measurement and analysis procedures Fig. 5.12 shows the measurement cycle adopted for the study of the X-ray dose and electric field dependence. On the top is shown, as a function of the measurement time, $t$, the applied gate voltage $V_{\text {gate }}(t)$, and on the bottom, the measured drain-source current $I_{d s}(t)$. In the pre-irradiation cycle, $V_{\text {gate }}$ is changed from accumulation to inversion, kept at inversion for 3 hours, and then changed back to accumulation, $V_{a c c}$. After 3 hours at $V_{a c c}$, this sequence is repeated. Three sequences are used, in order to check the reproducibility of the measurement results. In the irradiation cycle, $V_{\text {gate }}$ is biased to $V_{a c c}^{i r r}$, left at this voltage for 3 hours, followed by the X-ray irradiation, after which $V_{\text {gate }}$ is changed back to $V_{i n v}$. The irradiation cycle is followed by the post-irradiation cycle. The values of $V_{i n v}, V_{a c c}$ for the pre- and post-irradiation cycle are chosen so that the electric field in the oxide $E_{\text {field }}^{i n v} \approx-500 \mathrm{kV} / \mathrm{cm}$, and $E_{\text {field }}^{\text {post }} \approx 500 \mathrm{kV} / \mathrm{cm}$ respectively. In addition the values of $V_{\text {acc }}, V_{\text {acc }}^{\text {irr }}$ are chosen so that the electric field in the oxide before $E_{\text {field }}^{\text {pre irr }}$, and during the irradiation $E_{\text {field }}^{\text {irr }}$ is $\approx 500 \mathrm{kV} / \mathrm{cm}$. The electric field in accumulation is defined as $E_{\text {field }}=\left(V_{a c c}-V_{t h}\right) / t_{o x}$.

The p-MOSFET has been irradiated to a total ionizing dose of $16.61 \mathrm{kGy}\left(\mathrm{SiO}_{2}\right)$ in steps of $10,100,500,1 \mathrm{k}$ and $5 \mathrm{kGy}$ with a dose rate of $0.2 \mathrm{~Gy} / \mathrm{s}$, and a last step of $10 \mathrm{kGy}$ with a dose rate of $0.3 \mathrm{~Gy} / \mathrm{s}$. During the measurements and the storage, the p-MOSFET was kept at a temperature of about $23{ }^{\circ} \mathrm{C}$. From the measured 
$I_{d s}\left(V_{\text {gate }}\right)$ before and after X-ray irradiation, for which $V_{\text {gate }}$ was changed from $V_{a c c}$ to $V_{i n v}$, the values of $V_{t h}$, $\mu_{0}$ and $V_{1 / 2}$ are obtained using Eq. 5.1. From the $V_{t h}$ values the $N_{o x}^{e f f}$ is calculated using Eq. 5.5. During the irradiation with $V_{\text {gate }}$ in accumulation the p-MOSFET is in the off state and an electron accumulation layer is present below the gate, Fig. 5.14. Thus no channel current flows, so the time and dose dependence of $N_{o x}^{e f f}$ cannot be measured. Fig. 5.13 (a) shows the $I_{d s}\left(V_{\text {gate }}\right)$ calibration curves together with the fits for the different irradiations.

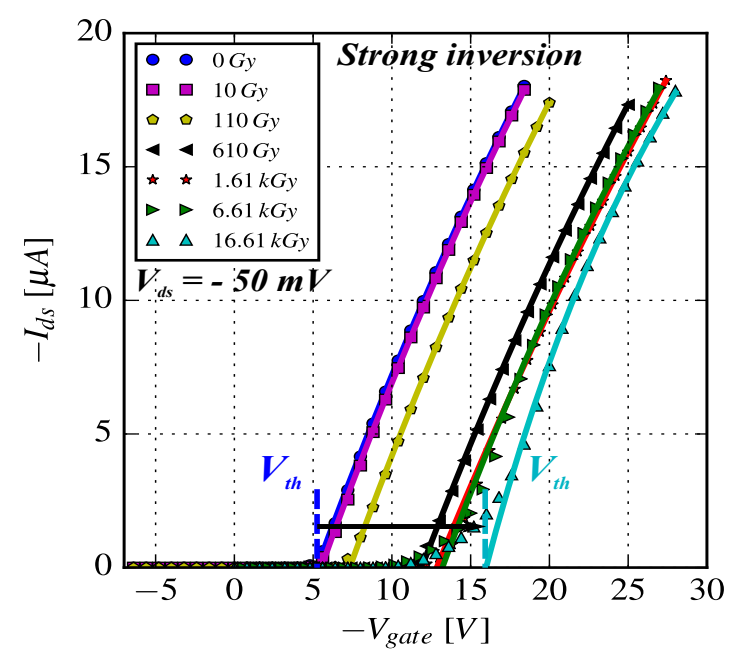

(a)

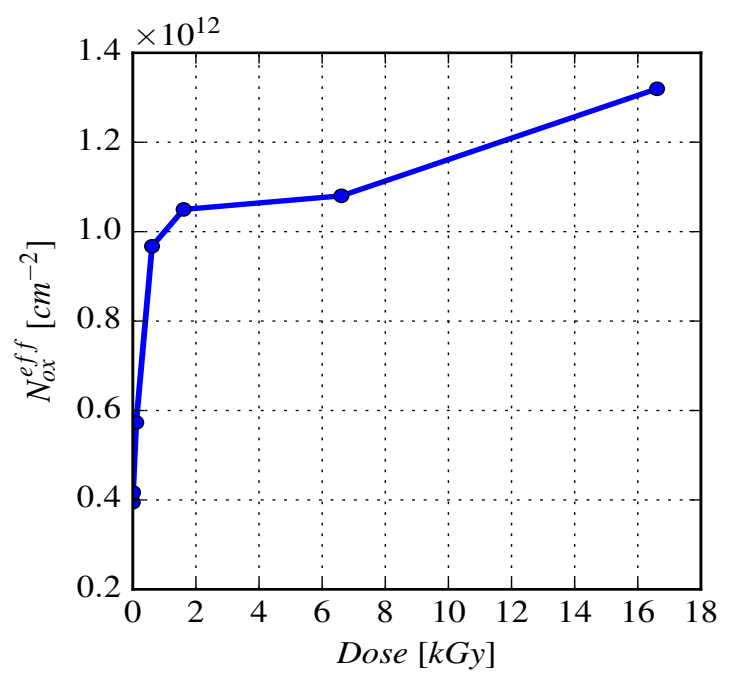

(b)

Figure 5.13: (a) $I_{d s}$ as a function of $V_{\text {gate }}$ in the linear region, and the results of the fits used to determine $V_{t h}$ and $\mu_{0}$ of p-MOSFET for the different X-ray dose values, and (b) Effective oxide charge density, $N_{o x}^{e f f}$ as a function of X-ray dose of p-MOSFET irradiated with $E_{\text {field }} \downarrow$.

It is found that the values of $V_{t h}$ change from $\approx-5 \mathrm{~V}$ to $\approx-16 \mathrm{~V}$ due to the increase of positive oxide charges. The slope of the $I_{d s}\left(V_{\text {gate }}\right)$ calibration curves is approximately constant with dose except for the last case of $16.61 \mathrm{kGy}$. The deviation of the $I_{d s}\left(V_{\text {gate }}\right)$ curves from linearity for large negative $V_{\text {gate }}$ values is due to the decrease of the hole mobility with increasing $\left|E_{\text {field }}\right|$ at the $\mathrm{Si}_{-} \mathrm{SiO}_{2}$ interface. In addition, Fig. 5.12 shows after every forward calibration, a decrease of $I_{d s}$ with time at the constant gate voltage $V_{\text {gate }}=V_{i n v}$. The time constant is of the order of 30 minutes. A decrease of $I_{d s}$ corresponds to an increase of $N_{o x}^{e f f}$. For $V_{\text {gate }}=V_{i n v}$ the p-MOSFET is in strong inversion: at the $\mathrm{Si}_{-} \mathrm{SiO}_{2}$ interface a hole inversion layer has formed, and the $E_{\text {field }}$ points $\uparrow$ from the $\mathrm{Si}$ into the $\mathrm{SiO}_{2}$, Fig. 5.7. As a result, border traps are charged up positively. As seen in Fig. 5.12, the value of $I_{d s}$ at the start of the reverse calibration is lower than the value at the end of the forward calibration. This corresponds to a reduction of $N_{o x}^{e f f}$. For $V_{\text {gate }} \gg V_{t h}$, the p-MOSFET is in accumulation: at the $\mathrm{Si}-\mathrm{SiO}_{2}$ interface an electron accumulation layer has formed, and the $E_{\text {field }}$ points $\downarrow$ from the $\mathrm{SiO}_{2}$ into the $\mathrm{Si}$. As a result, border traps are discharged.

Table 5.3 presents for the individual irradiation steps the integrated dose values, the values of $V_{i n v}$ applied to the gate for the post-irradiation cycle, the values of $V_{a c c}^{i r r}$ applied to the gate during the irradiation cycle, the electric field for the post-irradiation cycle in inversion, $E_{\text {field }}^{i n v}$, the electric field for the pre- and postirradiation cycles in accumulation, $E_{\text {field }}^{\text {pre irr }}$ and $E_{\text {field }}^{\text {post }}$. The average electric field during the irradiation in accumulation was $E_{\text {field }}^{i r r}$. The results for the effective oxide charge density and the hole mobility are 
Table 5.3: Irradiation conditions and extracted values of the surface radiation parameters as a function of X-ray dose of the p-MOSFET $<111>$ irradiated with $E_{\text {field }} \downarrow$.

\begin{tabular}{|c|c|c|c|c|c|c|c|c|c|c|c|c|}
\hline $\begin{array}{c}\text { Dose } \\
{[\mathrm{Gy}]}\end{array}$ & $\begin{array}{l}V_{i n v} \\
{[\mathrm{~V}]}\end{array}$ & $\begin{array}{l}V_{a c c}^{i r r} \\
{[\mathrm{~V}]}\end{array}$ & $\begin{array}{c}E_{\text {field }}^{\text {inv }} \\
{[\mathrm{kV} / \mathrm{cm}]}\end{array}$ & $\begin{array}{c}E_{\text {field }}^{\text {pre irr }} \\
{[\mathrm{kV} / \mathrm{cm}]}\end{array}$ & $\begin{array}{c}E_{\text {field }}^{\text {irr }} \\
{[\mathrm{kV} / \mathrm{cm}]}\end{array}$ & $\begin{array}{l}E_{\text {field }}^{\text {post irr }} \\
{[\mathrm{kV} / \mathrm{cm}]}\end{array}$ & $\begin{array}{l}N_{o x, \text { dose }}^{\text {eff }} \\
{\left[\mathrm{cm}^{-2}\right]}\end{array}$ & $\begin{array}{l}\Delta N_{o x, c h} \\
{\left[\mathrm{~cm}^{-2}\right]}\end{array}$ & $\begin{array}{c}\Delta N_{\text {ox }, \text { disch }} \\
{\left[\mathrm{cm}^{-2}\right]}\end{array}$ & $\begin{array}{c}\mu_{0} \\
{\left[\mathrm{~cm}^{2} /(\mathrm{Vs})\right]}\end{array}$ & $\begin{array}{l}V_{1 / 2} \\
{[\mathrm{~V}]}\end{array}$ & $\begin{array}{c}\Delta \\
{[\mathrm{nm}]}\end{array}$ \\
\hline 0 & -19.0 & 7.0 & -550 & 490 & 490 & 490 & $39.5 \cdot 10^{10}$ & $4.3 \cdot 10^{10}$ & $4.3 \cdot 10^{10}$ & 258 & -74 & 0.79 \\
\hline 10 & -19.0 & 7.0 & -540 & 490 & 500 & 500 & $41.7 \cdot 10^{10}$ & $3.9 \cdot 10^{10}$ & $4.5 \cdot 10^{10}$ & 261 & -72 & 0.81 \\
\hline 110 & -20.0 & 7.0 & -510 & 500 & 540 & 570 & $57.3 \cdot 10^{10}$ & $4.1 \cdot 10^{10}$ & $5.8 \cdot 10^{10}$ & 259 & -71 & 0.83 \\
\hline 610 & -25.0 & 6.0 & -520 & 530 & 520 & 520 & $96.7 \cdot 10^{10}$ & $4.5 \cdot 10^{10}$ & $9.5 \cdot 10^{10}$ & 247 & -77 & 0.76 \\
\hline 1610 & -28.6 & 1.0 & -630 & 510 & 460 & 410 & $105.0 \cdot 10^{10}$ & $4.7 \cdot 10^{10}$ & $10.8 \cdot 10^{10}$ & 230 & -96 & 0.61 \\
\hline 6610 & -28.0 & 0 & -590 & 500 & 510 & 530 & $108.0 \cdot 10^{10}$ & $6.6 \cdot 10^{10}$ & $25.0 \cdot 10^{10}$ & 255 & -61 & 0.96 \\
\hline 16610 & -28.0 & 0 & -480 & 510 & 570 & 640 & $131.9 \cdot 10^{10}$ & $7.4 \cdot 10^{10}$ & $31.7 \cdot 10^{10}$ & 357 & -23 & 2.49 \\
\hline
\end{tabular}

presented. Fig. 5.13 (b) presents the values of $N_{o x}^{e f f}$ as a function of X-ray dose, $N_{o x, d o s e}^{e f f}(D)$ in the eighth column of Table 5.3. These values were determined at the end of each irradiation cycle. Before irradiation, $N_{\text {ox, dose }}^{\text {eff }}(0)=4.0 \cdot 10^{11} \mathrm{~cm}^{-2}$, which is in the range expected for $\mathrm{Si}$ with $<111>$ orientation. For the maximum dose of $16.61 \mathrm{kGy}\left(\mathrm{SiO}_{2}\right), N_{\text {ox }}^{\text {effose }}(16.61 \mathrm{kGy})=1.3 \cdot 10^{12} \mathrm{~cm}^{-2}$. From the values shown in the Table 5.3 as well as from Fig. 5.13 (b), one sees the $N_{o x, \text { dose }}^{e f f}(D)$ shows an increase of $\approx 60 \%$ up to $1.61 \mathrm{kGy}$, however after $610 \mathrm{~Gy}$ the $\mathrm{d} N_{o x, \text { dose }}^{e f f}(D) / \mathrm{d} D$ decreases rapidly with dose. Probably the low $V_{\text {gate }}$ bias $1.0,0$ and $0 \mathrm{~V}$ for the last three irradiation steps, decreases the rate of positive oxide charge build-up at the $\mathrm{Si}_{-} \mathrm{SiO}_{2}$ interface.

Border traps and hole mobility Border traps communicate with the Si over a wide range of time scales. The ninth column of Table 5.3 shows the X-ray dose dependence of $\Delta N_{o x, c h}(D)$, the charging up of the border traps during the three hours after irradiation, and the tenth column $\Delta N_{o x, \text { disch }}(D)$, the discharging of the border traps, when changing $V_{\text {gate }}$ from inversion to accumulation, remaining 3 hours in accumulation and then biasing back to inversion. A significant charging up and discharging of border traps, at the level of $6-$ $24 \%$ of $N_{o x}^{e f f}$, is observed. For the highest dose values, the biggest values of $\Delta N_{o x, c h}(D)$ and of $\Delta N_{o x, d i s c h}(D)$

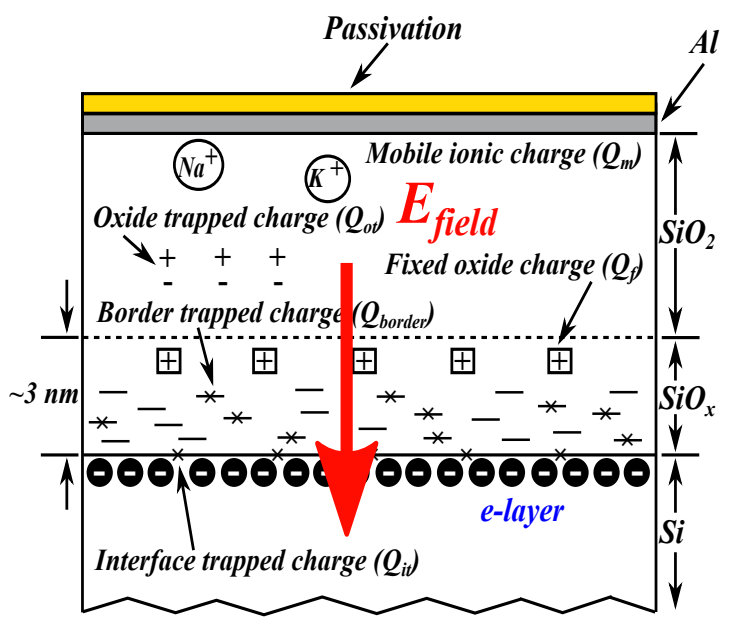

Figure 5.14: Nomenclature of oxide charges, electron accumulation layer and $E_{\text {field }} \downarrow$ direction during irradiation. 


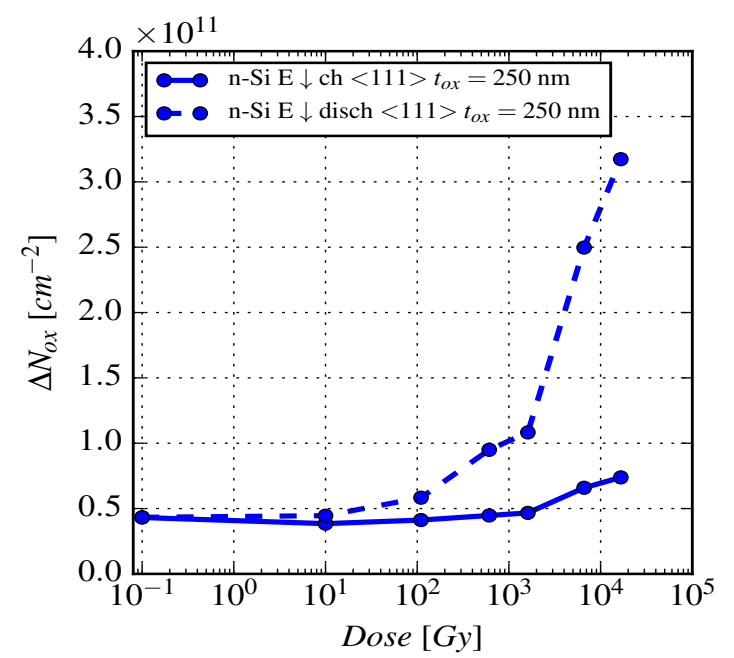

(a)

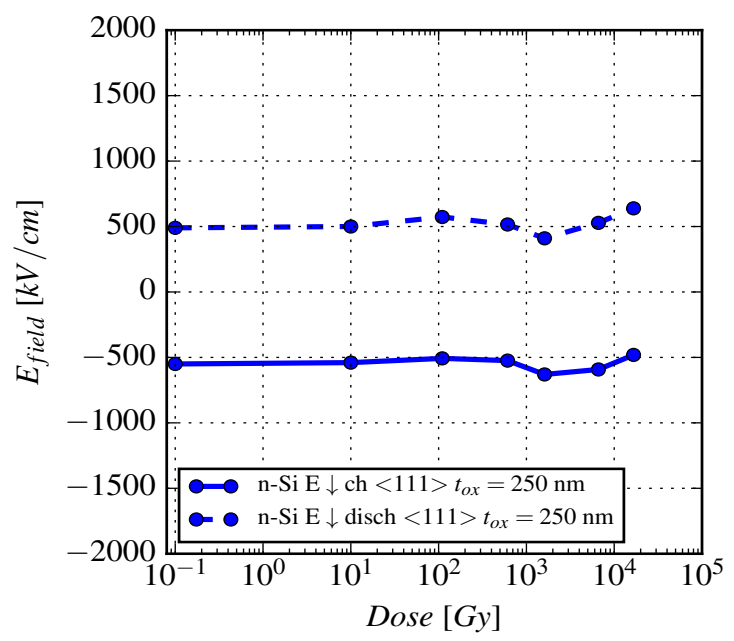

(b)

Figure 5.15: (a) $\Delta N_{o x}$ of charging and discharging of border traps, and (b) $E_{\text {field }}$ values during charging and discharging of border traps as a function of the accumulated X-ray dose of p-MOSFET irradiated with $E_{\text {field }}$ $\downarrow$.

are observed, shown Fig. 5.15 (a). The electric field during charging up, $E_{\text {field }}^{\text {inv }}$, and discharging, $E_{\text {field }}^{\text {post irr }}$ is chosen $\approx-500 \mathrm{kV} / \mathrm{cm}$ and $\approx 500 \mathrm{kV} / \mathrm{cm}$ respectively, as shown in Fig. 5.15 (b).

Furthermore, the $I_{d s}\left(V_{\text {gate }}\right)$ calibrations allow to determine the dependence of the hole mobility on $V_{\text {gate }}$ for different dose values. For the parametrization Eq. 5.2 is used. The results for $\mu_{0}$ and $V_{1 / 2}$ for the reverse calibration curves following the irradiations, are shown in Table 5.3 and in Fig. 5.16. For the non-irradiated p-MOSFET, a hole mobility at $E_{\text {field }} \approx 0$ of $\mu_{0}=258 \mathrm{~cm}^{2} /(\mathrm{V} \cdot \mathrm{s})$ is extracted, which is significantly smaller

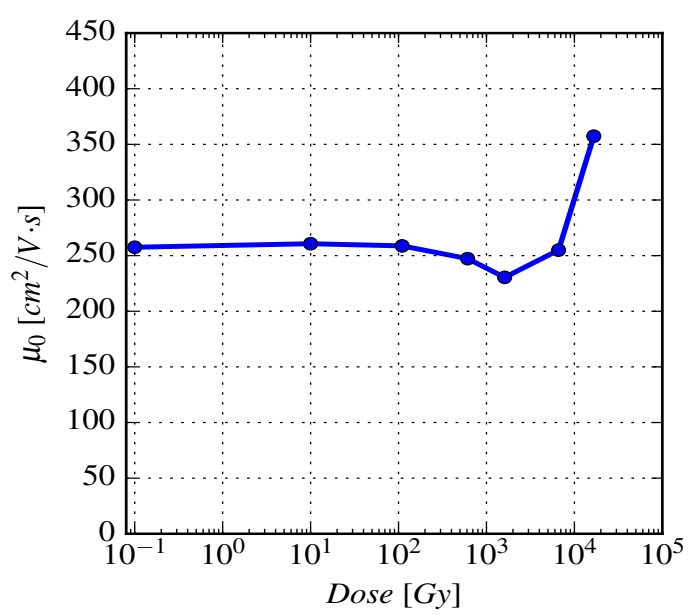

(a)

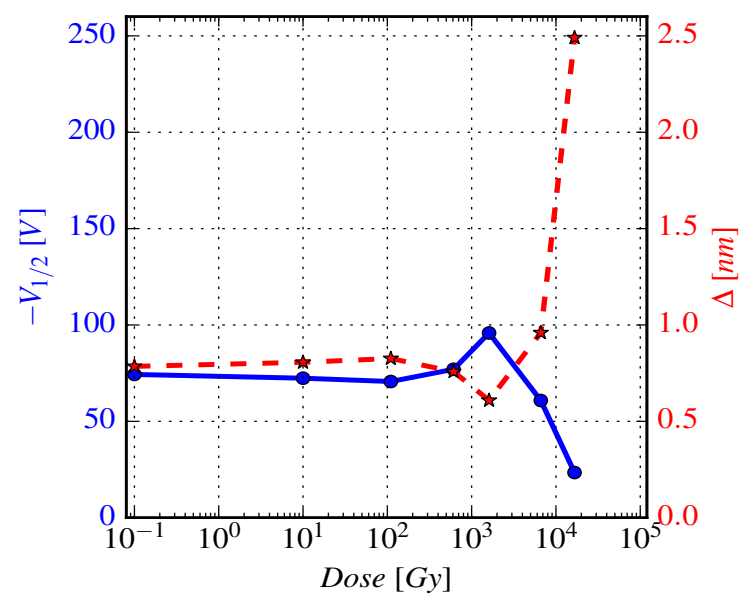

(b)

Figure 5.16: (a) Hole mobility, $\mu_{0}$, and (b) $V_{1 / 2}$ and $\Delta$ as a function of the accumulated X-ray dose of p-MOSFET irradiated with $E_{\text {field }} \downarrow$. 
than the bulk mobility of $\approx 450 \mathrm{~cm}^{2} /(\mathrm{V} \cdot \mathrm{s})$, similar to the $\mu_{0}$ of the p-MOSFET presented in the previous section. The mobility $\mu_{0}$ stays approximately constant with increasing dose, however for the highest dose increases in contrast to the previous case. The value of $V_{1 / 2}$ changes with dose from $-74 \mathrm{~V}$ to about $-23 \mathrm{~V}$. In Fig. 5.16 (b) is also presented the dose dependence of the surface roughness amplitude, $\Delta$, which is defined by Eq. 5.6.

\subsubsection{The n-MOSFET irradiated with $E_{\text {field }}$ pointing from the $\mathrm{Si}$ into the $\mathrm{SiO}_{2}$}

Similar to the methodology presented in the previous sections, irradiations on a n-MOSFET with the $E_{\text {field }}$ pointing $\uparrow$ from the $\mathrm{Si}$ into the $\mathrm{SiO}_{2}$, have been performed. A circular n-MOSFET with the dimensions presented above, has been used. The test structure biased in accumulation at a field in the $\mathrm{SiO}_{2}$ of about 500 $\mathrm{kV} / \mathrm{cm}$ during irradiation and irradiated by $\mathrm{X}$-rays up to a dose of about $17 \mathrm{kGy}\left(\mathrm{SiO}_{2}\right)$ in different irradiation steps. Before and after each irradiation, the gate voltage has been cycled from inversion to accumulation conditions and back and the threshold voltage and the electron mobility at the $\mathrm{Si}_{-} \mathrm{SiO}_{2}$ interface determined.

Test structure For the present study, a circular n-MOSFET, fabricated by Hamamatsu on $<100>$ p-type Magnetic Czochralski (MCz) Si with a bulk doping of $N_{d}=4.4 \cdot 10^{12} \mathrm{~cm}^{-3}$, has been used. Fig. 5.17 shows a cross section and a top view of the n-MOSFET. The width over length ratio $W / L=2 \pi / \ln \left(r_{2} / r_{1}\right) \approx 4.96$, where $r_{1}=99 \mu \mathrm{m}$ is the inner and $r_{2}=351 \mu \mathrm{m}$ the outer radius of the FET channel, respectively. The thermally grown $\mathrm{SiO}_{2}$ has a thickness $t_{o x} \approx 700 \mathrm{~nm}$. The n-MOSFET has been part of a test field processed together with a number of different silicon sensors, Fig. 5.17.

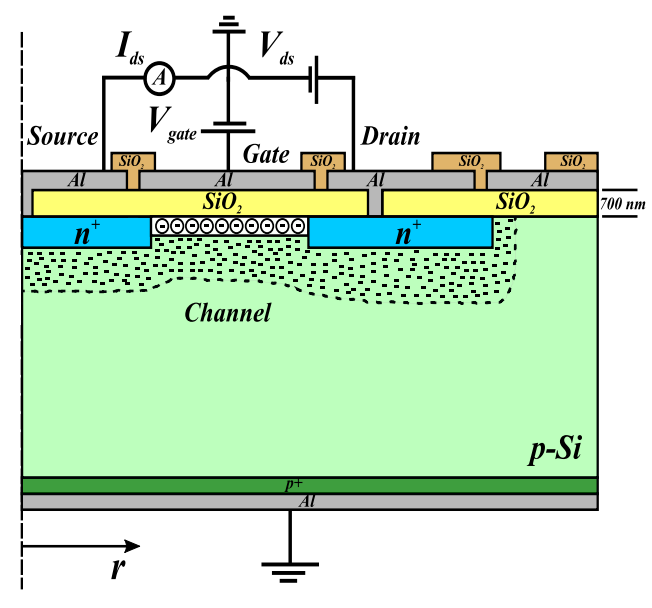

(a)

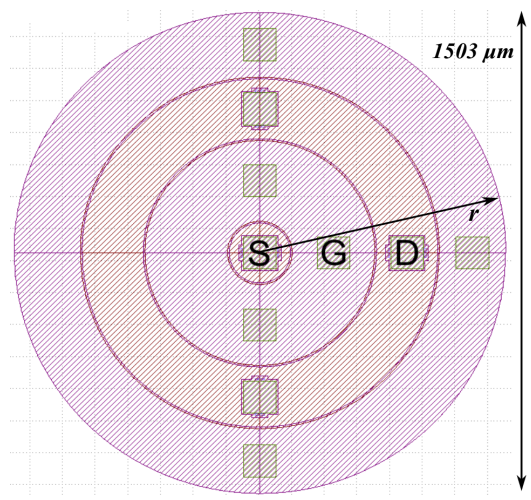

(b)

Figure 5.17: (a) Measurement setup, and cross section and (b) top view of the n-MOSFET $<100>$.

Measurement and analysis procedures Fig. 5.18 shows the measurement cycle adopted for the study of the X-ray dose and electric field dependence. On the top is shown, as a function of the measurement time, $t$, the applied gate voltage $V_{\text {gate }}(t)$, and on the bottom, the measured drain-source $I_{d s}(t)$. 


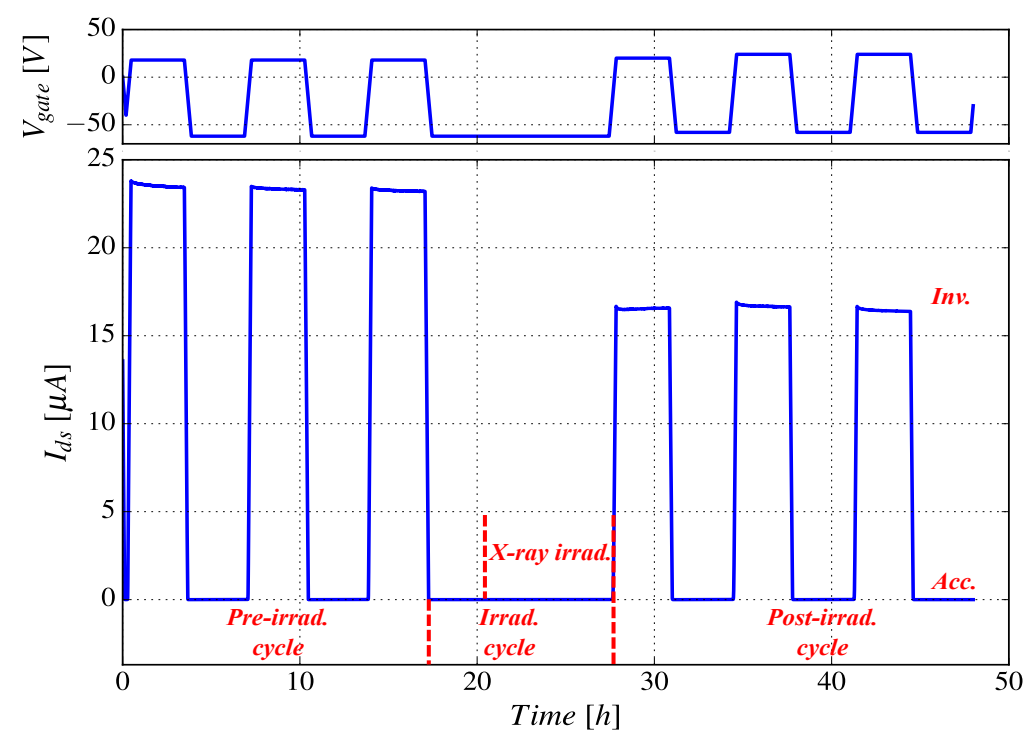

Figure 5.18: Measurement cycle: the applied gate voltage, $V_{\text {gate }}(t)$, is shown on the top, and the measured drain-source current, $I_{d s}(t)$, on the bottom of n-MOSFET irradiated at $5 \mathrm{kGy}$ X-ray dose with $E_{\text {field }} \uparrow$.

In the pre-irradiation cycle, $V_{\text {gate }}$ is changed from accumulation to inversion, kept at inversion for 3 hours, and then changed back to accumulation, $V_{a c c}$. After 3 hours at $V_{a c c}$, this sequence is repeated. Three sequences are used, in order to check the reproducibility of the measurement results. In the irradiation cycle, $V_{\text {gate }}$ is biased to $V_{a c c}^{i r r}$, left at this voltage for 3 hours, followed by the X-ray irradiation, after which $V_{\text {gate }}$ is changed back to $V_{i n v}$. The irradiation cycle is followed by the post-irradiation cycle. The values of $V_{i n v}, V_{a c c}$ for the pre- and post-irradiation cycle are chosen so that the electric field in the oxide $E_{\text {field }}^{i n v} \approx 500 \mathrm{kV} / \mathrm{cm}$ and $E_{\text {field }}^{\text {post }} \approx-500 \mathrm{kV} / \mathrm{cm}$ respectively. The values of $V_{a c c}, V_{\text {acc }}^{\text {irr }}$ are chosen properly so that the electric field in the oxide before $E_{\text {field }}^{\text {pre irr }}$, and during the irradiation $E_{\text {field }}^{\text {irr }}$ is $\approx-500 \mathrm{kV} / \mathrm{cm}$.

The n-MOSFET has been irradiated to a total ionizing dose of $16.61 \mathrm{kGy}\left(\mathrm{SiO}_{2}\right)$ in steps of $10,100,500,1 \mathrm{k}$ and $5 \mathrm{kGy}$ with a dose rate of $0.2 \mathrm{~Gy} / \mathrm{s}$, and a last step of $10 \mathrm{kGy}$ with a dose rate of $0.3 \mathrm{~Gy} / \mathrm{s}$. During the measurements and the storage, the n-MOSFET was kept at a temperature of about $23{ }^{\circ} \mathrm{C}$. From the measured $I_{d s}\left(V_{\text {gate }}\right)$ before and after X-ray irradiation, for which $V_{\text {gate }}$ was changed from $V_{a c c}$ to $V_{i n v}$, the values of $V_{t h}$, $\mu_{0}$ and $V_{1 / 2}$ are obtained using Eq. 5.1. From $V_{t h}$, the effective oxide charge density, $N_{o x}^{e f f}$, is obtained by Eq. 5.5 with $(+)$ for p-bulk Si where $\phi_{b}=0.1647 \mathrm{~V}$ has been used for the potential difference between the midgap potential and the Fermi level in the $\mathrm{Si}$, and $\phi_{m s}=-0.7029 \mathrm{~V}$ for the Al-Si work function difference.

During the irradiation with $V_{\text {gate }}$ in accumulation the n-MOSFET is in the off state. A hole accumulation layer is present below the gate, Fig. 5.20, and no channel current flows, thus the time and dose dependence of $N_{o x}^{e f f}$ cannot be measured. Fig. 5.19 shows the $I_{d s}\left(V_{\text {gate }}\right)$ calibration curves together with the fits for the different irradiations. It is found that the values of $V_{t h}$ change from $\approx-2 \mathrm{~V}$ to $\approx-38 \mathrm{~V}$ up to X-ray dose of $610 \mathrm{~Gy}$, and then $V_{t h}$ increases, reaches $\approx 1 \mathrm{~V}$ for the higher irradiation step. The observed decrease of the slope of the $I_{d s}\left(V_{\text {gate }}\right)$ curves from linearity for large $\left|V_{\text {gate }}\right|$ values is attributed to the decrease of the electron mobility with increasing $E_{\text {field }}$ at the $\mathrm{Si}-\mathrm{SiO}_{2}$ interface.

Next is presented the time dependence of the drain-source current, $I_{d s}(t)$, which is shown in Fig. 5.18 for the $5 \mathrm{kGy}$ irradiation. In the post-irradiation cycle after every forward calibration, $I_{d s}$ shows a small decrease with time and afterwards starts to increase at the constant gate voltage $V_{\text {gate }}=V_{i n v}$ for 3 hours. An increase of 


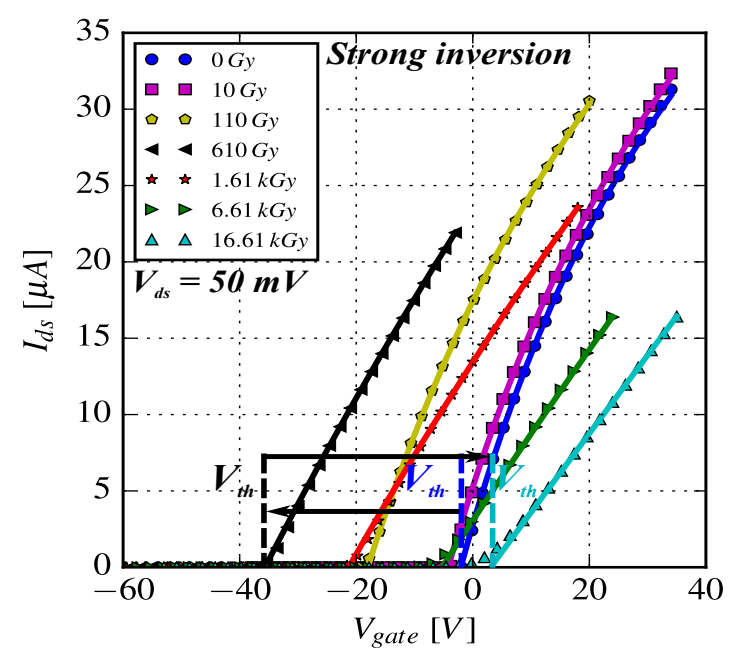

(a)

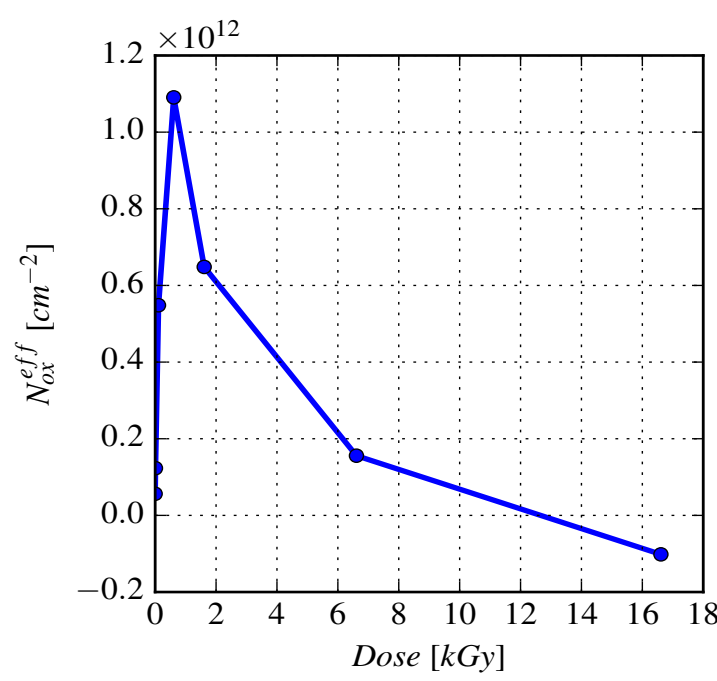

(b)

Figure 5.19: (a) $I_{d s}$ as a function of $V_{\text {gate }}$ in the linear region, and the results of the fit used to determine $V_{t h}$ and $\mu_{0}$ of n-MOSFET for the different X-ray dose values, and (b) Effective oxide charge density, $N_{o x}^{e f f}$ as a function of X-ray dose of n-MOSFET irradiated with $E_{\text {field }} \uparrow$.

$I_{d s}$ corresponds to a decrease of $N_{o x}^{e f f}$. For $V_{g a t e}=V_{i n v}$ the n-MOSFET is in strong inversion: at the $\mathrm{Si}_{-} \mathrm{SiO}_{2}$ interface an electron inversion layer has formed, and the electric field $E_{f i e l d}=\left(V_{i n v}-V_{t h}\right) / t_{o x}$ points $\downarrow$ from the $\mathrm{SiO}_{2}$ into the $\mathrm{Si}$. With this $E_{\text {field }}$ direction, border traps discharge if they are charged up positively as presented in the previous section or charged up with negative charge due to electron trapping. In both cases the $N_{o x}^{e f f}$ decreases. The increase of the density of charged border traps is defined as $\Delta N_{o x, c h}=N_{o x}^{e f f}\left(t_{0}+\right.$ $3 \mathrm{~h})-N_{o x}^{e f f}\left(t_{0}\right)$, where $t_{0}$ is the time at which $V_{\text {gate }}=V_{i n v}$ was reached for the forward calibration. For $\left|V_{\text {gate }}\right| \ll\left|V_{t h}\right|$, the n-MOSFET is in accumulation: at the $\mathrm{Si}_{-} \mathrm{SiO}_{2}$ interface a hole accumulation layer is present, and the electric field points $\uparrow$ from the $\mathrm{Si}$ into $\mathrm{SiO}_{2}$, Fig. 5.20. In this $E_{\text {field }}$ direction border traps are charged up due to hole trapping or electron de-trapping. In both cases the $N_{o x}^{\text {eff }}$ increases. The decrease of the density of charged border traps defined as $\Delta N_{o x, \text { disch }}=N_{o x, \text { forw }}^{e f f}-N_{o x, \text { rev }}^{\text {eff }}$. Table 5.4 presents for the individual irradiation steps the integrated dose values, the values of $V_{i n v}$ applied to the gate for the postirradiation cycle, the values of $V_{a c c}^{i r r}$ applied to the gate during the irradiation cycle, the electric field for the post-irradiation cycle in inversion, $E_{\text {field }}^{\text {inv }}$, the electric field for the pre- and post-irradiation cycles in accumulation, $E_{\text {field }}^{\text {pre irr }}$ and $E_{\text {field }}^{\text {post irr }}$.

The average electric field during the irradiation in accumulation was $E_{\text {field }}^{i r r}$. The results for the effective oxide charge density and the electron mobility are presented. Fig. 5.19 (b) presents the values of $N_{o x}^{e f f}$ as a function of X-ray dose, $N_{o x, d o s e}^{e f f}(D)$ in the eighth column of Table 5.4. These values were determined at the end of each irradiation cycle. Before irradiation, $N_{o x, \text { dose }}^{\text {eff }}(0)=5.6 \cdot 10^{10} \mathrm{~cm}^{-2}$, which is in the range expected for $\mathrm{Si}$ with $<100>$ orientation. The effective oxide charge increases up to $610 \mathrm{~Gy}$ and then start to decreases up to $16.61 \mathrm{kGy}$. For the maximum dose of $16.61 \mathrm{kGy}\left(\mathrm{SiO}_{2}\right)$ a negative effective oxide charge is measured, $N_{\text {ox }, \text { dose }}^{\text {eff }}(16.61 \mathrm{kGy})=-10.2 \cdot 10^{10} \mathrm{~cm}^{-2}$. 
Table 5.4: Irradiation conditions and extracted values of the surface radiation parameters as a function of X-ray dose of the n-MOSFET $<100>$ irradiated with $E_{\text {field }} \uparrow$.

\begin{tabular}{|c|c|c|c|c|c|c|c|c|c|c|c|c|}
\hline $\begin{array}{c}\text { Dose } \\
{[\mathrm{Gy}]}\end{array}$ & $\begin{array}{l}V_{i n v} \\
{[\mathrm{~V}]}\end{array}$ & $\begin{array}{l}V_{a c c}^{i r r} \\
{[\mathrm{~V}]}\end{array}$ & $\begin{array}{c}E_{\text {field }}^{\text {inv }} \\
{[\mathrm{kV} / \mathrm{cm}]}\end{array}$ & $\begin{array}{c}E_{\text {field }}^{\text {pre irr }} \\
{[\mathrm{kV} / \mathrm{cm}]}\end{array}$ & $\begin{array}{c}E_{\text {field }}^{\text {irr }} \\
{[\mathrm{kV} / \mathrm{cm}]}\end{array}$ & $\begin{array}{l}E_{\text {field }}^{\text {post irr }} \\
{[\mathrm{kV} / \mathrm{cm}]}\end{array}$ & $\begin{array}{l}N_{o x, \text { dose }}^{\text {eff }} \\
{\left[\mathrm{cm}^{-2}\right]}\end{array}$ & $\begin{array}{l}\Delta N_{o x, c h} \\
{\left[\mathrm{~cm}^{-2}\right]}\end{array}$ & $\begin{array}{c}\Delta N_{o x, \text { disch }} \\
{\left[\mathrm{cm}^{-2}\right]}\end{array}$ & $\begin{array}{c}\mu_{0} \\
{\left[\mathrm{~cm}^{2} /(\mathrm{Vs})\right]}\end{array}$ & $\begin{array}{l}V_{1 / 2} \\
{[\mathrm{~V}]}\end{array}$ & $\begin{array}{c}\Delta \\
{[\mathrm{nm}]}\end{array}$ \\
\hline 0 & 34.0 & -38.0 & 520 & -510 & -510 & -510 & $5.6 \cdot 10^{10}$ & $-0.1 \cdot 10^{10}$ & $0.3 \cdot 10^{10}$ & 1148 & 56 & 1.94 \\
\hline 10 & 34.0 & -38.0 & 550 & -510 & -500 & -480 & $12.3 \cdot 10^{10}$ & $0.3 \cdot 10^{10}$ & 0 & 1083 & 66 & 1.66 \\
\hline 110 & 20.0 & -40.0 & 540 & -520 & -520 & -530 & $54.8 \cdot 10^{10}$ & $0.1 \cdot 10^{10}$ & $4.7 \cdot 10^{10}$ & 974 & 77 & 1.42 \\
\hline 610 & -3.0 & -55.0 & 470 & -530 & -600 & -680 & $109.1 \cdot 10^{10}$ & $-1.2 \cdot 10^{10}$ & $17.0 \cdot 10^{10}$ & 609 & 292 & 0.37 \\
\hline 1610 & 18.0 & -76.0 & 560 & -600 & -590 & -580 & $64.8 \cdot 10^{10}$ & $-0.4 \cdot 10^{10}$ & $13.8 \cdot 10^{10}$ & 550 & 321 & 0.34 \\
\hline 6610 & 24.0 & -62.0 & 420 & -600 & -680 & -750 & $15.6 \cdot 10^{10}$ & $-2.5 \cdot 10^{10}$ & $8.7 \cdot 10^{10}$ & 477 & 696 & 0.16 \\
\hline 16610 & 35.0 & -50.0 & 460 & -640 & -740 & -830 & $-10.2 \cdot 10^{10}$ & $-0.5 \cdot 10^{10}$ & $8.7 \cdot 10^{10}$ & 426 & 2628 & 0.04 \\
\hline
\end{tabular}

Border traps and electron mobility The ninth column of Table 5.4 shows the X-ray dose dependence of $\Delta N_{o x, c h}(D)$, the charging up of the border traps during the three hours after irradiation, and the tenth column $\Delta N_{o x, \text { disch }}(D)$, the discharging of the border traps, when changing $V_{\text {gate }}$ from inversion to accumulation, remaining 3 hours in accumulation and then biasing back to inversion. The values of $\Delta N_{o x, c h}(D)$ are positive and negative and the $\left|\Delta N_{o x, c h}(D)\right| \ll\left|N_{o x}^{e f f}\right|$. The negative values of $\Delta N_{o x, c h}(D)$ correspond to discharging of border traps or charging up with negative charge due to electron trapping, as the electric field points $\downarrow$ from the $\mathrm{SiO}_{2}$ into the $\mathrm{Si}$. Similar the positive values of $\Delta N_{o x, \text { disch }}(D)$ correspond to discharging of border traps due to hole de-trapping or charging due to electron trapping. As the electric field points $\uparrow$ from the $\mathrm{Si}$ into the $\mathrm{SiO}_{2}$, the electron trapping is possible to happen from the bulk $\mathrm{SiO}_{2}$ to the $\mathrm{Si}_{-} \mathrm{SiO}_{2}$ interface. The values of $\Delta N_{o x, d i s c h}(D)$ are at the level of $\approx 15 \%$ of the $N_{o x}^{e f f}$. The $\Delta N_{o x, c h}(D), \Delta N_{o x, \text { disch }}(D)$ and the electric field during charging, $E_{\text {field }}^{\text {inv }}$, and discharging, $E_{\text {field }}^{\text {post irr }}$ is chosen $\approx 500 \mathrm{kV} / \mathrm{cm}$ and $\approx-500 \mathrm{kV} / \mathrm{cm}$ respectively, as shown in Fig. 5.21.

In addition, the $I_{d s}\left(V_{\text {gate }}\right)$ calibrations allow to determine the dependence of the electron mobility on $V_{\text {gate }}$ for different dose values. For the parametrization Eq. 5.2 is used. The results for $\mu_{0}$ and $V_{1 / 2}$ for the reverse calibration curves following the irradiations, are shown in Table 5.4 and in Fig. 5.22. For the non-irradiated

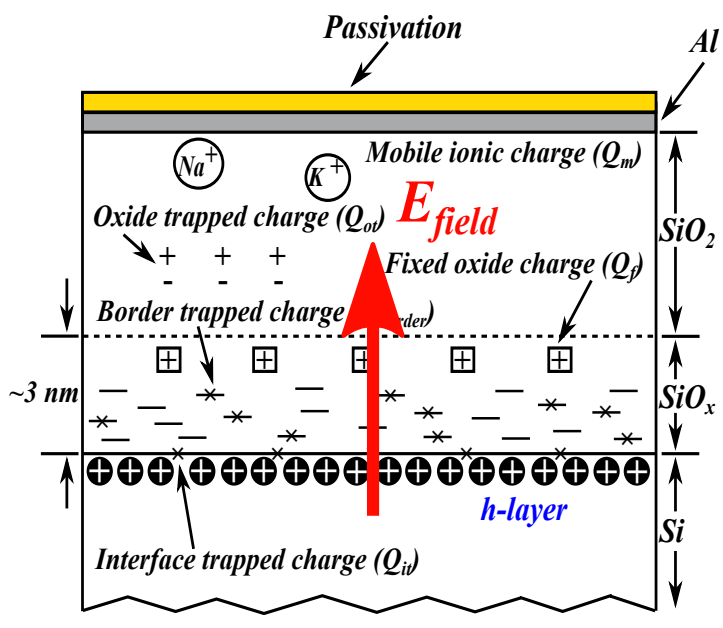

Figure 5.20: Nomenclature of oxide charges, hole accumulation layer and $E_{\text {field }} \uparrow$ direction during irradiation. 


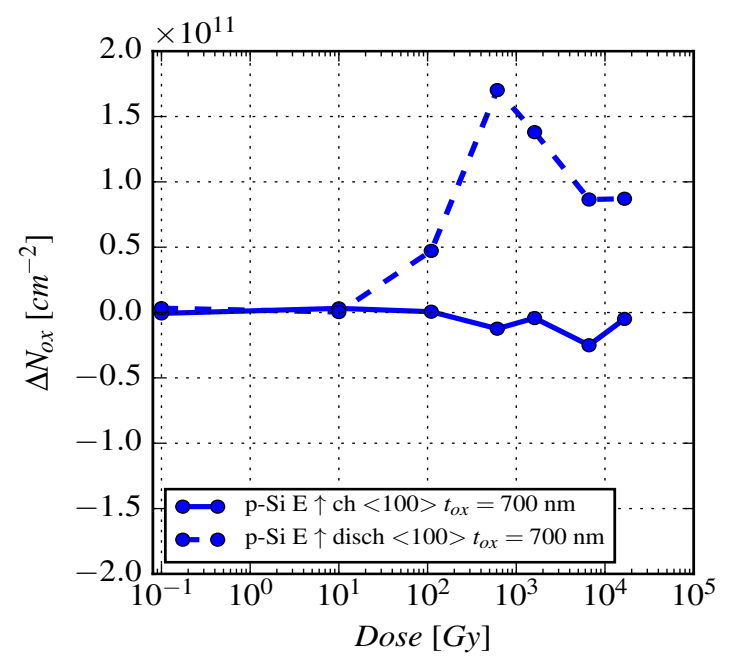

(a)

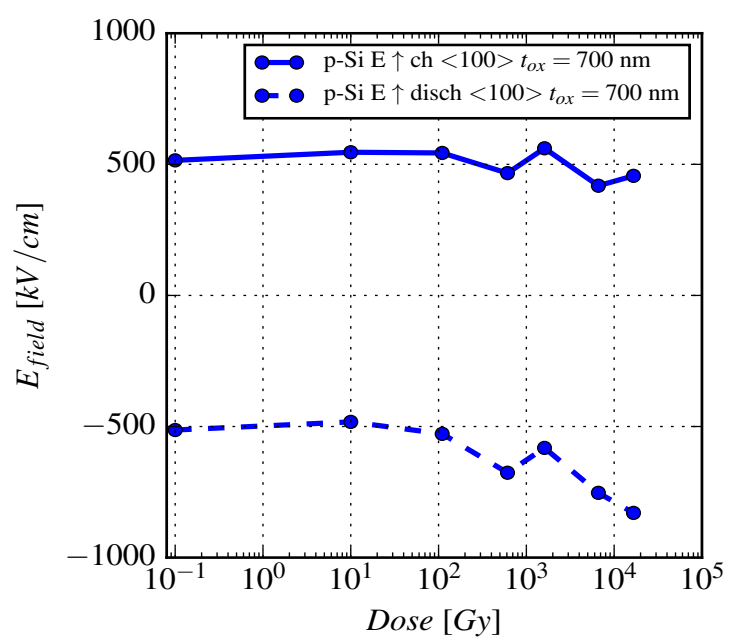

(b)

Figure 5.21: (a) $\Delta N_{o x}$ of charging and discharging of border traps, and (b) $E_{\text {field }}$ values during charging and discharging of border traps as a function of the accumulated X-ray dose of n-MOSFET irradiated with $E_{\text {field }}$ $\uparrow$.

n-MOSFET, is extracted an electron mobility at $E_{\text {field }} \approx 0$ of $\mu_{0}=1148 \mathrm{~cm}^{2} /(\mathrm{V} \cdot \mathrm{s})$, which is significantly smaller than the bulk electron mobility of $\approx 1450 \mathrm{~cm}^{2} /(\mathrm{V} \cdot \mathrm{s})$. As expected $\mu_{0}$ decreases with increasing dose. The value of $V_{1 / 2}$ changes with dose from $56 \mathrm{~V}$ to about $2628 \mathrm{~V}$. In Fig. 5.22 (b) is also presented the dose dependence of the surface roughness amplitude, $\Delta$, which is defined by Eq. 5.6 with the weighting factor $\eta$ equal to $1 / 2$ for electrons.

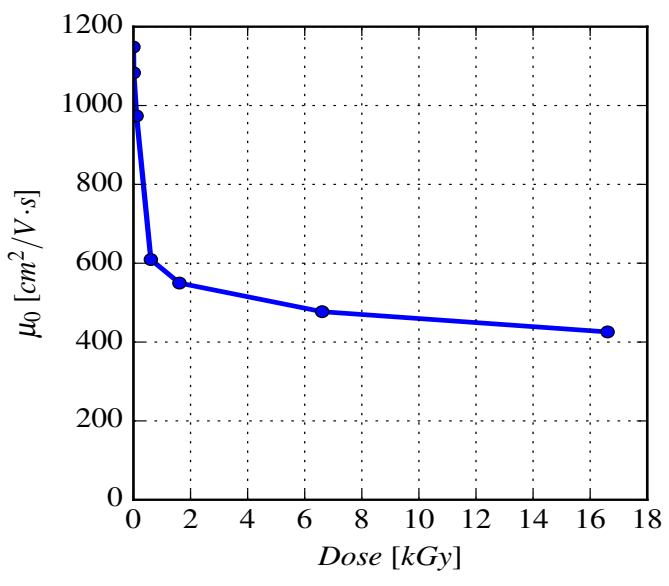

(a)

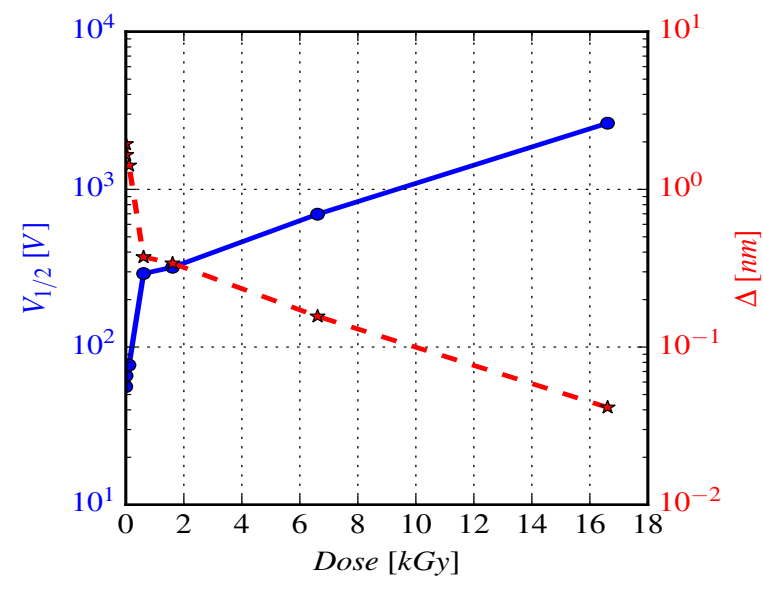

(b)

Figure 5.22: (a) Electron mobility, $\mu_{0}$, and (b) $V_{1 / 2}$ and $\Delta$ as a function of the accumulated X-ray dose of n-MOSFET irradiated with $E_{\text {field }} \uparrow$. 


\subsubsection{The n-MOSFET irradiated with $E_{\text {field }}$ pointing from the $\mathrm{SiO}_{2}$ into the $\mathrm{Si}$}

Similar to the methodology presented for the previous three cases, irradiations on a n-MOSFET with $E_{\text {field }}$ pointing $\downarrow$ from the $\mathrm{SiO}_{2}$ into the $\mathrm{Si}$, have been performed. A circular n-MOSFET with the same dimensions as in Fig. 5.17, fabricated by Hamamatsu on $<100>$ p-type Magnetic Czochralski (MCz) Si with the same process and bulk doping of $N_{d}=4.4 \cdot 10^{12} \mathrm{~cm}^{-3}$, has been used. The test structure biased in inversion at a field in the $\mathrm{SiO}_{2}$ of about $500 \mathrm{kV} / \mathrm{cm}$ in the beginning of the irradiation and irradiated by X-rays to a dose of about $6.61 \mathrm{kGy}\left(\mathrm{SiO}_{2}\right)$ in different irradiation steps. Before and after each irradiation, the gate voltage has been cycled from inversion to accumulation conditions and back and the threshold voltage and the electron mobility at the $\mathrm{Si}_{-} \mathrm{SiO}_{2}$ interface determined.

Measurement and analysis procedures Fig. 5.23 shows the measurement cycle adopted for the study of the X-ray dose and electric field dependence of surface radiation damage. On the top is shown, as a function of the measurement time, $t$, the applied gate voltage $V_{\text {gate }}(t)$, and on the bottom, the measured drain-source current $I_{d s}(t)$.

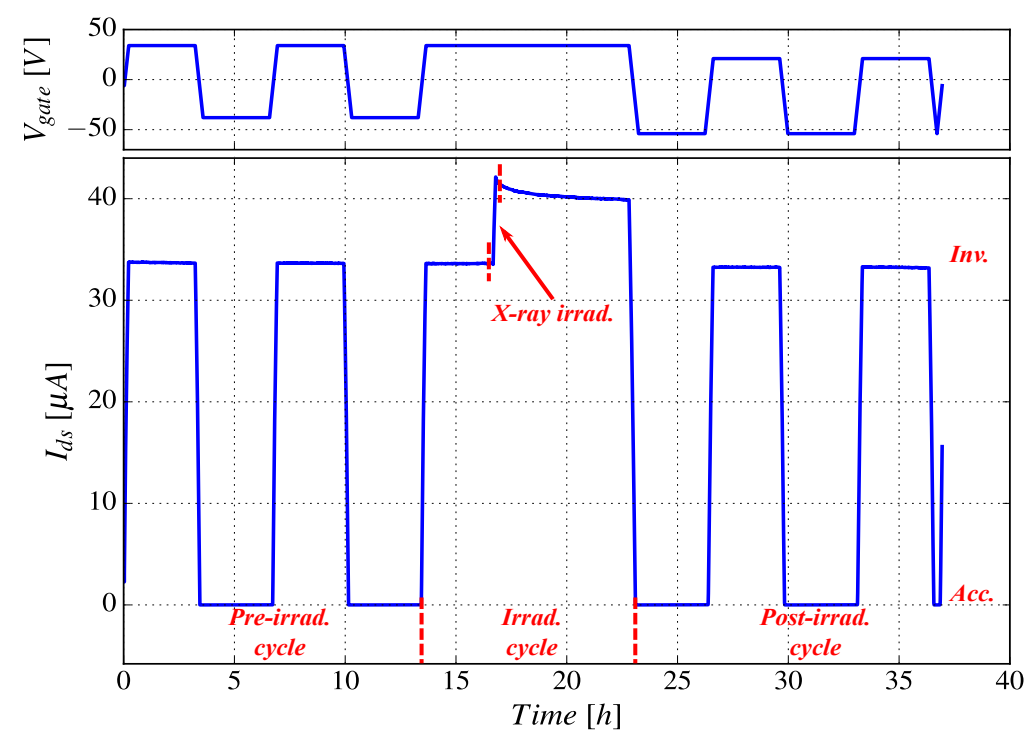

Figure 5.23: Measurement cycle: the applied gate voltage, $V_{\text {gate }}(t)$, is shown on the top, and the measured drain-source current, $I_{d s}(t)$, on the bottom of n-MOSFET irradiated at 80 Gy X-ray dose with $E_{\text {field }} \downarrow$.

In the pre-irradiation cycle, $V_{g a t e}$ is changed from $V_{a c c}$ to $V_{i n v}$, kept at $V_{i n v}$ for 3 hours, and then changed back to $V_{a c c}$. After 3 hours $V_{a c c}$, this sequence is repeated. Two sequences are used, in order to check the reproducibility of the measurement results. In the irradiation cycle, $V_{\text {gate }}$ is again changed to $V_{i n v}$, left at this voltage for 3 hours, followed by the X-ray irradiation and another 6 hours at $V_{i n v}$, after which $V_{\text {gate }}$ is changed back to $V_{a c c}$. The irradiation cycle is followed by the post-irradiation cycle. The value of $V_{i n v}$ for the pre-irradiation and the irradiation cycle is chosen so that the electric field in the oxide $E_{\text {field }}^{\text {pre irr }} \approx 500 \mathrm{kV} / \mathrm{cm}$. The build-up of positive charges during the irradiation, results in a change of $V_{t h}$, and thus of $E_{\text {field }}$. In order to apply approximately the same $E_{\text {field }}$ value in the post-irradiation cycle, $V_{i n v}$ is changed and the corresponding electric field is called $E_{\text {field }}^{\text {post irr }}$. The n-MOSFET has been irradiated to a total ionizing dose of $6.61 \mathrm{kGy}\left(\mathrm{SiO}_{2}\right)$ in steps of $10,10,10,80,80,420,100,290,250 \mathrm{~Gy}$ and $5.36 \mathrm{kGy}$ with a dose rate of $0.2 \mathrm{~Gy} / \mathrm{s}$. During the measurements and the storage, the n-MOSFET was kept at a temperature of about 
$23{ }^{\circ} \mathrm{C}$. For the measured $I_{d s}\left(V_{\text {gate }}\right)$ before and after X-ray irradiation, for which $V_{\text {gate }}$ was changed from $V_{a c c}$ to $V_{i n v}$, the values of $V_{t h}, \mu_{0}$ and $V_{1 / 2}$ of the forward calibration are obtained using Eq. 5.1, and the reverse calibration when changing $V_{\text {gate }}$ from $V_{i n v}$ to $V_{a c c}$. Using Eq. 5.5 with (+) for p-bulk $\mathrm{Si}, N_{o x, \text { forw }}^{e f f}$ and $N_{o x, \text { rev }}^{e f f}$ is calculated from the $V_{t h}$ values. In order to determine $N_{o x}^{e f f}(t)$, similar to the p-MOSFET described in the previous section when $V_{g a t e}=V_{i n v}$, Eq. 5.1 is inverted to calculate $V_{t h}(t)$ from the measured $I_{d s}(t)$, Eq. 5.7.

For the part before irradiation the extracted parameters $\mu_{0}^{\text {pre }}$ and $V_{1 / 2}^{\text {pre }}$ from the $I_{d s}\left(V_{\text {gate }}\right)$ calibration curve measured in the reverse direction have been used. For the part after irradiation the extracted parameters $\mu_{0}^{\text {post }}$ and $V_{1 / 2}^{\text {post }}$ from the calibration curve measured in the reverse direction have been used. For the part during irradiation, the linear interpolation of the $V_{t h}$ change of the regions before and after irradiation has been assumed, in order to describe $V_{t h}(t)$. In this way $N_{o x}^{e f f}(t)$ is measured also during the irradiation, as the electron mobility changes. Fig. 5.24 (a) shows the $I_{d s}\left(V_{\text {gate }}\right)$ calibration curves together with the fits for the different irradiations.

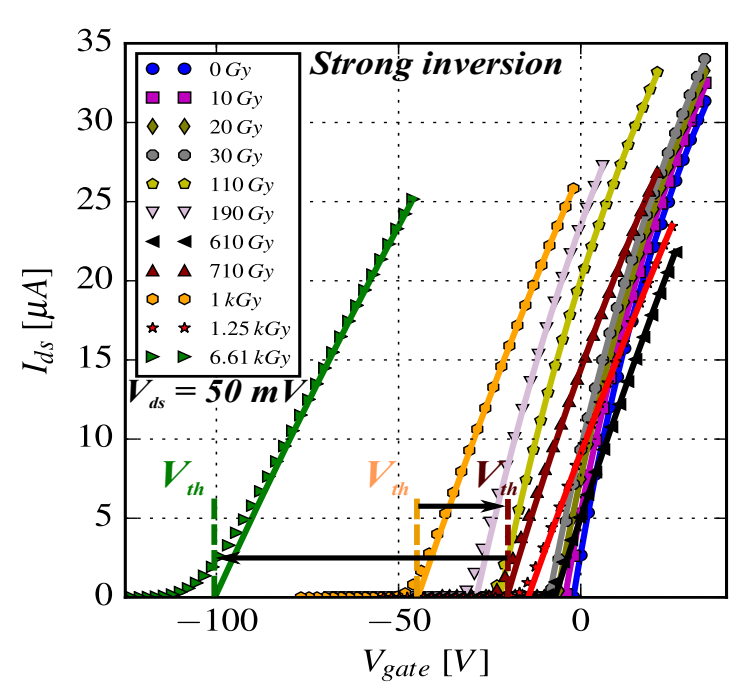

(a)

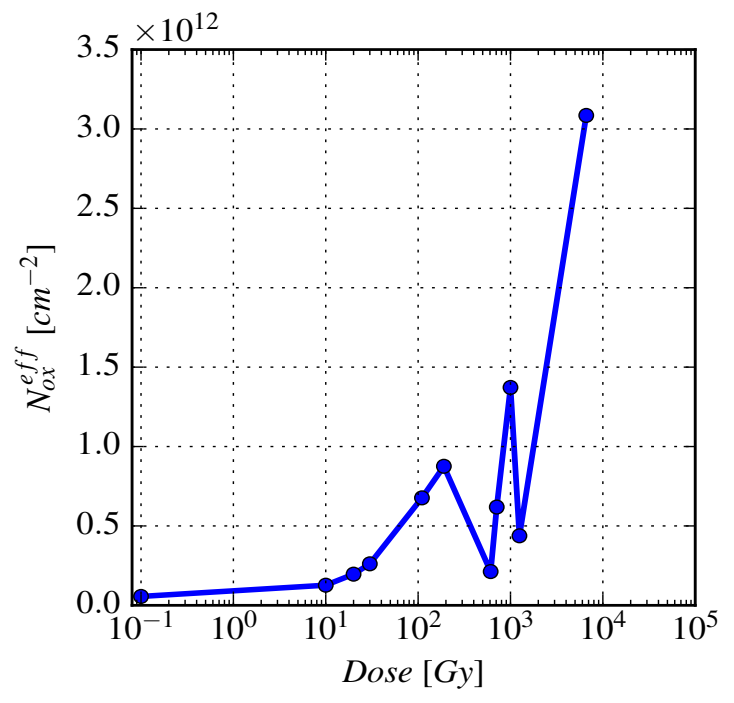

(b)

Figure 5.24: (a) $I_{d s}$ as a function of $V_{\text {gate }}$ in the linear region, and the results of the fit used to determine $V_{t h}$ and $\mu_{0}$ of n-MOSFET for the different X-ray dose values, and (b) Effective oxide charge density, $N_{o x}^{e f f}$ as a function of X-ray dose of n-MOSFET irradiated with $E_{\text {field }} \downarrow$.

It is found that the values of $V_{t h}$ change from $\approx-2 \mathrm{~V}$ to $\approx-100.0 \mathrm{~V}$ due to the increase of positive oxide charges. The observed decrease of the slope of the $I_{d s}\left(V_{\text {gate }}\right)$ curves with dose is the result of the reduction of the electron mobility. The deviation of the $I_{d s}\left(V_{\text {gate }}\right)$ curves from linearity for large $\left|V_{\text {gate }}\right|$ values is due to the decrease of the electron mobility with increasing $E_{\text {field }}$ at the $\mathrm{Si}_{-} \mathrm{SiO}_{2}$ interface. However as it is shown in Fig. 5.24 (a) the threshold voltage after two irradiation steps 420 Gy and 250 Gy shifts to the positive direction. This corresponds to a decrease of the $N_{o x}^{e f f}$ as it is shown in Fig. 5.24 (b). The measurement cycle for these two steps is shown in Fig. 5.25. According to Refs. [100,101,135,136] when the bias voltage during irradiation is changed from positive to negative, the direction of electron and hole processes are reversed. Holes move to the metal gate, or are trapped so close to the gate that cause a negligible $V_{t h}$ shift and electrons move toward the $\mathrm{Si}-\mathrm{SiO}_{2}$ interface. If an electron encounters a hole trapped near the $\mathrm{Si}_{-} \mathrm{SiO}_{2}$ interface, the electron may become trapped nearby and neutralize the charge of the hole, or recombine with the trapped 


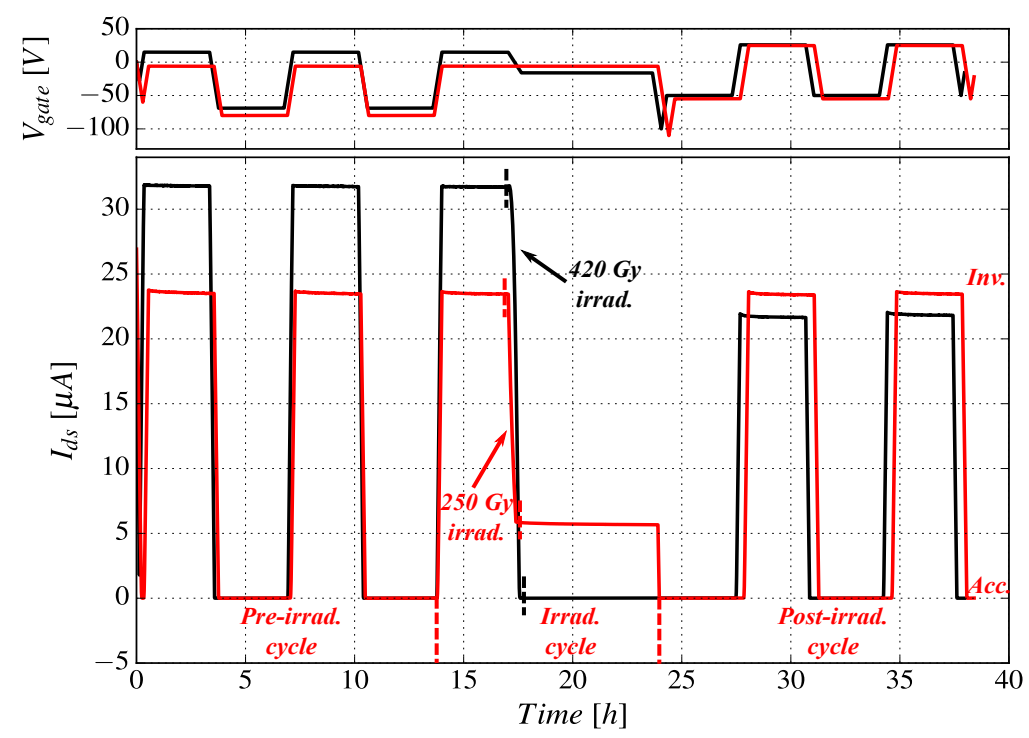

Figure 5.25: Measurement cycle: the applied gate voltage, $V_{\text {gate }}(t)$, is shown on the top, and the measured drain-source current, $I_{d s}(t)$, on the bottom of n-MOSFET irradiated at $420 \mathrm{~Gy}$ and $250 \mathrm{~Gy} \mathrm{X}$-ray dose with negative $V_{\text {gate }}$.

hole, reducing the $N_{o x}^{e f f}$ in either case. In particular as presented in Table 5.5 for the 80 Gy irradiation step between $110 \mathrm{~Gy}$ and $190 \mathrm{~Gy}$ total dose, the gate voltage in inversion during irradiation, $V_{i n v}^{i r r}$, was ramped from $21 \mathrm{~V}$ to $6 \mathrm{~V}$ and the $N_{o x}^{e f f}$ increased from $\approx 6.8 \cdot 10^{11} \mathrm{~cm}^{-2}$ to $\approx 8.8 \cdot 10^{11} \mathrm{~cm}^{-2}$.

As expected and shown in Fig. 5.26 (a) for positive $V_{\text {gate }}$, the electric field in the oxide, $E_{o x}=V_{\text {gate }} / t_{o x}$, and the electric field at the interface $E_{\text {field }}=\left(V_{\text {gate }}-V_{t h}\right) / t_{o x}$ are pointing $\downarrow$ from the $\mathrm{SiO}_{2}$ to the $\mathrm{Si}$.

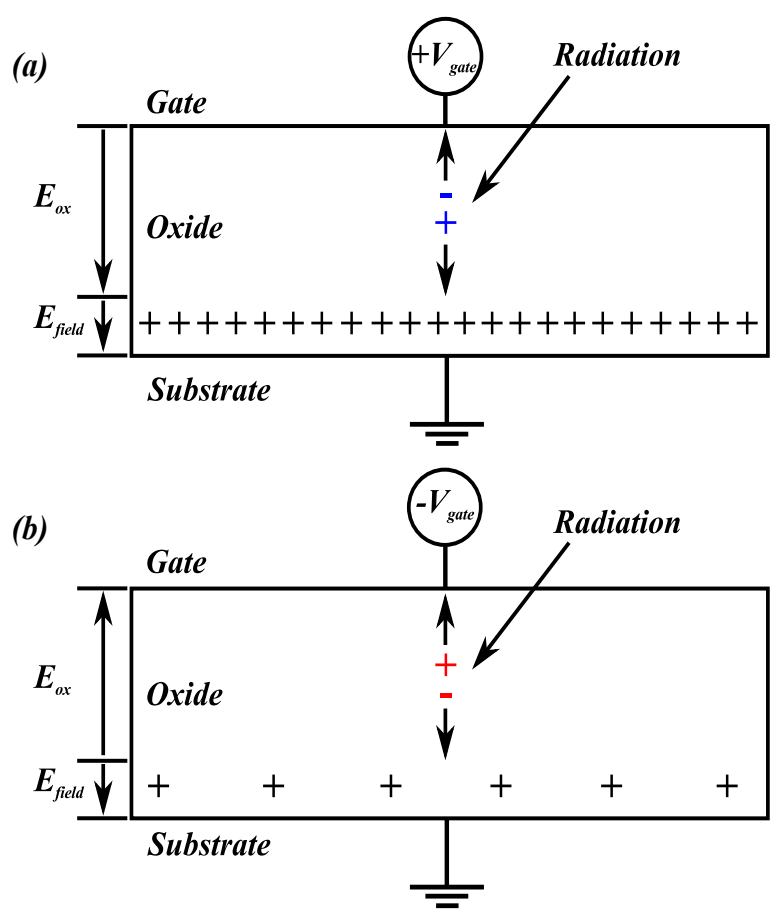

Figure 5.26: Electric field direction in the oxide, $E_{o x}$, and at the interface, $E_{\text {field }}$, for positive (a) and (b) negative gate voltage $V_{\text {gate }}$. 
Holes move to the interface and get trapped so the $N_{o x}^{e f f}$ increases. For the next irradiation step of $420 \mathrm{~Gy}$ the $V_{i n v}^{i r r}$ was ramped from $15 \mathrm{~V}$ to $-16 \mathrm{~V}$ and as shown in Fig. 5.26 (b), the $E_{o x}$ points $\uparrow$ from the Si to the $\mathrm{SiO}_{2}$ and the $E_{\text {field }}$ points $\downarrow$ from the $\mathrm{SiO}_{2}$ to the $\mathrm{Si}$. In this situation, the radiation-induced electrons move to the interface, where they are trapped causing a decrease of $N_{o x}^{e f f}$ from $\approx 8.8 \cdot 10^{11} \mathrm{~cm}^{-2}$ to $\approx 2.1 \cdot 10^{11} \mathrm{~cm}^{-2}$. The measurement of the $N_{o x}^{e f f}(t)$ during irradiation was possible at the starting point of the irradiation, after some time the n-MOSFET passed from "on" to "off" state, and the current flow below the gate stopped, due to the transition from inversion to accumulation. Also the charge neutralization effect observed when the gate voltage during irradiation $V_{i n v}^{i r r}$, was constant at $-6 \mathrm{~V}$, for the irradiation step of $250 \mathrm{~Gy}$ after $1 \mathrm{kGy}$ total dose. As shown in Fig. 5.24 (b) and Table 5.5 the $N_{o x}^{\text {eff }}$ decreased from $\approx 13.7 \cdot 10^{11} \mathrm{~cm}^{-2}$ to $\approx 4.4 \cdot 10^{11} \mathrm{~cm}^{-2}$. For this case, the irradiation stopped before the n-MOSFET change state and the measurements of the $N_{o x}^{e f f}(t)$ and $E_{\text {field }}(t)$ are shown in Fig. 5.27 with red color.

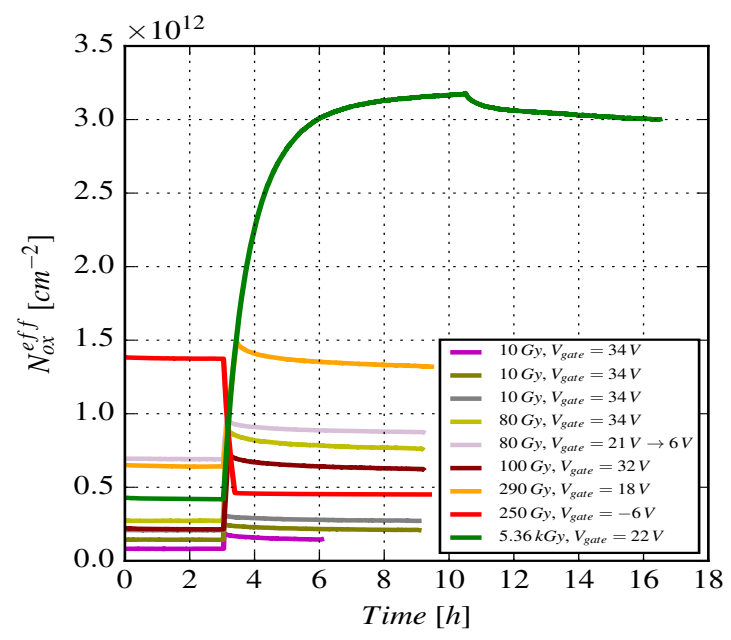

(a)

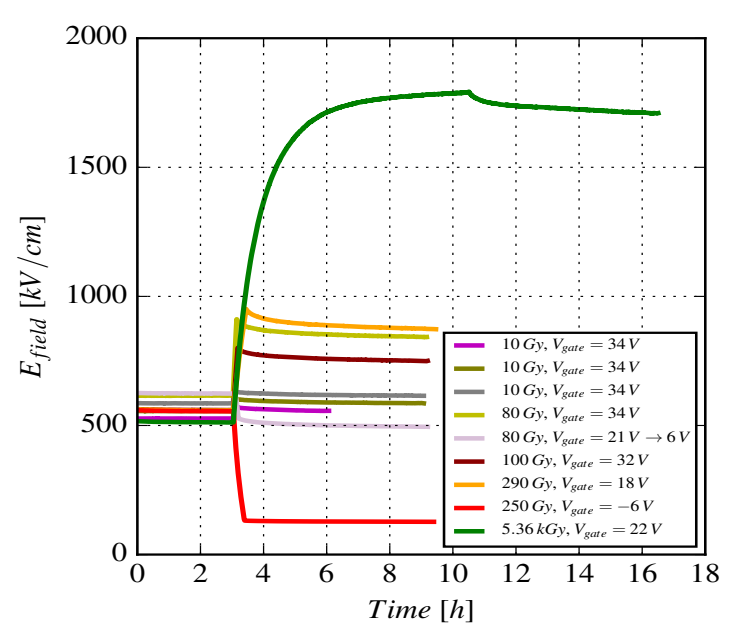

(b)

Figure 5.27: (a) Effective oxide charge density, $N_{o x}^{e f f}(t)$ and (b) Electric field $E_{\text {field }}$ as a function of time for the individual irradiation cycles of n-MOSFET irradiated with $E_{\text {field }} \downarrow$.

The electric field at the interface decreased from $E_{\text {field }}^{\text {pre irr }} \approx 560 \mathrm{kV} / \mathrm{cm}$ to $E_{\text {field }}^{\text {post irr }} \approx 140 \mathrm{kV} / \mathrm{cm}$ due to the decrease of the $N_{o x}^{e f f}$. Table 5.5 presents for the individual irradiation steps, the values of $V_{\text {gate }}$ in accumulation $V_{a c c}$, of $V_{i n v}^{i r r}$ applied to the gate for the pre-irradiation and irradiation cycle, the electric field in accumulation $E_{\text {field }}^{\text {acc }}$, the electric field for the pre- and post-irradiation cycles, $E_{\text {field }}^{\text {pre irr }}$ and $E_{\text {field }}^{\text {post }}$, the average electric field during the irradiation, $E_{\text {field }}^{i r r}$, and the results for the effective oxide charge density and the electron mobility. Fig. 5.27 (a) shows for the individual irradiation steps $N_{o x}^{e f f}(t)$ during the irradiation cycles, and the eighth column of Table 5.5 the values of $N_{o x, \text { dose }}^{\text {eff }}(D)$, the effective oxide charge density determined at the end of each irradiation cycle. Before irradiation, $N_{o x, \text { dose }}^{\text {eff }}(0)=5.6 \cdot 10^{10} \mathrm{~cm}^{-2}$, which is in the range expected for $\mathrm{Si}$ with $<100>$ orientation. For the maximum dose of $6.61 \mathrm{kGy}\left(\mathrm{SiO}_{2}\right)$, $N_{o x, \text { dose }}^{\text {eff }}(6.61 \mathrm{kGy})=3.1 \cdot 10^{12} \mathrm{~cm}^{-2}$. From the values shown in the Table 5.5 as well as from Fig. 5.27 (a), one sees that the derivative $\mathrm{d} N_{o x, \text { dose }}^{e f f}(D) / \mathrm{d} D$ decreases with dose. Fig. 5.27 (b) shows $E_{\text {field }}(t)$ during the irradiation cycles for the individual steps. The irradiations performed with constant and ramping $V_{\text {gate }}$, at the $V_{i n v}^{i r r}$ values of the third column of Table 5.5. So the $E_{f i e l d}$ was increasing during irradiation due to the increase of $N_{o x}^{e f f}$. 
Table 5.5: Irradiation conditions and extracted values of the surface radiation parameters as a function of X-ray dose of the n-MOSFET $<100>$ irradiated with $E_{\text {field }} \downarrow$.

\begin{tabular}{|c|c|}
\hline$\triangleleft \bar{\Xi}$ & 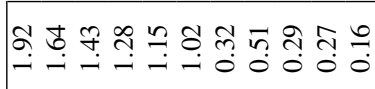 \\
\hline$\leq 2$ & 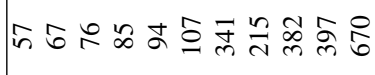 \\
\hline ₹ & 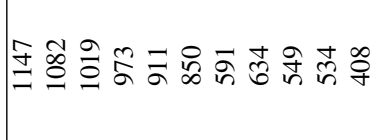 \\
\hline & 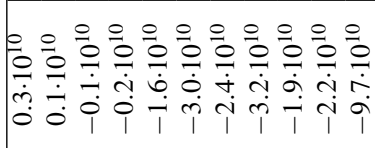 \\
\hline & 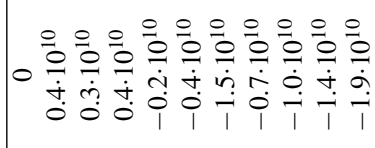 \\
\hline & 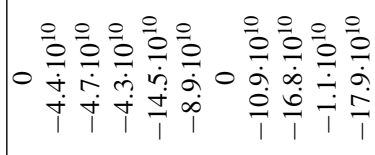 \\
\hline & 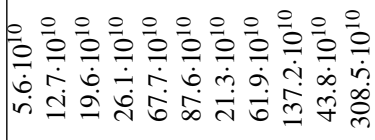 \\
\hline & 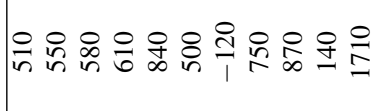 \\
\hline 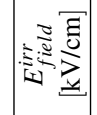 & 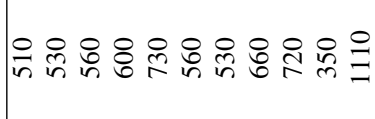 \\
\hline & 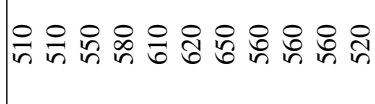 \\
\hline & 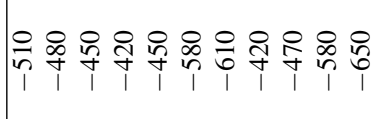 \\
\hline & 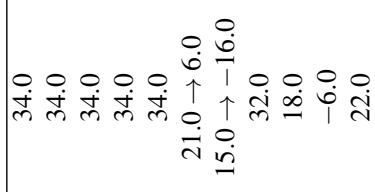 \\
\hline$\stackrel{\check{\Sigma}}{\nu}$ & 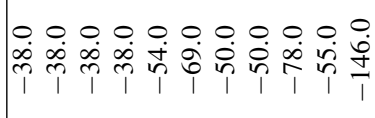 \\
\hline 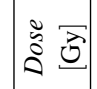 & ○ㅇํㅇㅇㅢ \\
\hline
\end{tabular}




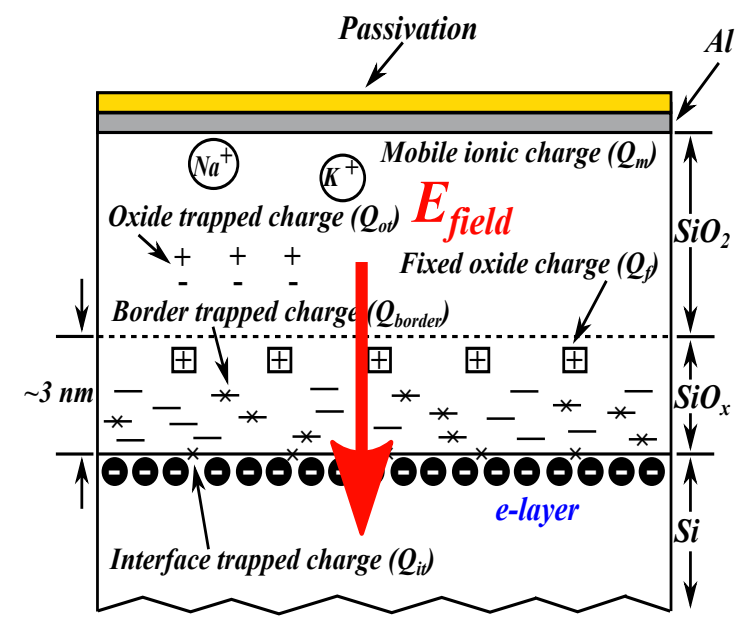

Figure 5.28: Nomenclature of oxide charges, electron inversion layer and $E_{\text {field }} \downarrow$ direction during irradiation.

The irradiation with field pointing $\downarrow$ from the $\mathrm{SiO}_{2}$ into the $\mathrm{Si}$ and positive $V_{\text {gate }}$, Fig. 5.28, maximizes the build-up of positive charges in the oxide. The ninth column of Table 5.5 shows the dose dependence of $\Delta N_{o x, \text { reb }}(D)$, the decreasing of $N_{o x}^{e f f}$ after the irradiation due to the recovery-"rebound" effect at constant bias after irradiation of the n-MOSFET. The $\Delta N_{o x, r e b}$ as a function of time for the individual irradiation steps is presented in Fig. 5.29 (a). The $\Delta N_{o x, r e b}(t)$ was measured for 6 hours except the first step of $10 \mathrm{~Gy}$, where it was measured for 3 hours.

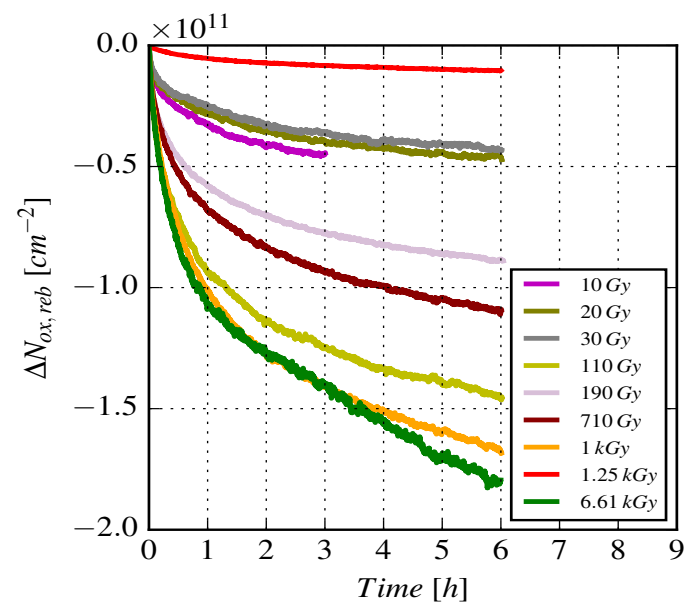

(a)

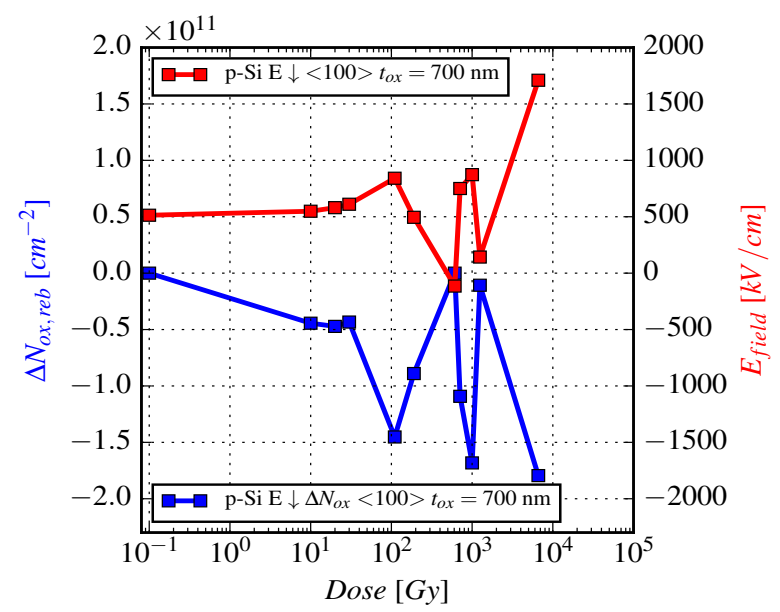

(b)

Figure 5.29: (a) $\Delta N_{o x, r e b}$ as a function of time after the individual irradiation steps, and (b) $\Delta N_{o x, r e b}$ and $E_{\text {field }}$ as a function of the accumulated X-ray dose of n-MOSFET irradiated with $E_{\text {field }} \downarrow$.

In Fig. 5.29 (b) is presented the $\Delta N_{o x, r e b}$ and $E_{\text {field }}^{\text {post irr }}$ as a function of the accumulated X-ray dose. As mentioned above the dose rate during irradiation was $0.2 \mathrm{~Gy} / \mathrm{s}\left(\mathrm{SiO}_{2}\right)$ and between the irradiation steps the n-MOSFET biased for many hours in inversion or in accumulation. It can be seen that time dependent 
effects during and after irradiation can complicate MOS hardness assurance testing and worst case response modeling [101, 137]. The duration of all the irradiation steps except the last of $5.36 \mathrm{kGy}$, was $<1$ hour. Assuming a time constant of the effect 3 hours no significant recovery observed during irradiation. However for the $5.36 \mathrm{kGy}$ irradiation step, green color in Fig. 5.27 (a) the time duration was $\approx 7.5$ hours. The shape of the curve indicates a large impact of recovery during the total X-ray dose irradiation. In particular the rate of the negative shift is approximately compensated by the recovery, Eq. 5.9,

$$
-\frac{d V_{\text {rad }}}{d t} \leq \frac{d V_{r e c}}{d t}
$$

where $d V_{\text {rad }} / d t$ is the rate that the radiation produces negative voltage shifts and $d V_{\text {rec }} / d t$ is the slope of the recovery curve [109]. As shown in Fig. 5.27 (a) for the first irradiation step of 10 Gy, magenta color, the recovery stops when gate bias is not present. The next irradiation of $10 \mathrm{~Gy}$, olive color, starts from the same $N_{o x}^{e f f}$ value where the previous recovery stopped. The recovery after the first irradiation steps is much greater, as the damage that introduced during these steps also recovers later. The peak in Figs. 5.27 (a) and (b) when the irradiation stops and the recovery starts is due to nearly exact cancellation of the two competing mechanisms that contribute to the shift in threshold voltage. As shown in Figs. 5.29 (a) and (b) the recovery for the higher irradiation steps is approximately the same. This indicates that the recovery properties are independent of the total X-ray dose. A dose rate dependence is expected [109], however all the irradiation steps were performed with one dose rate value and no further conclusions can be extracted. For negative bias during and after irradiation no significant recovery was observed, Fig. 5.27 (a). According to Refs. $[106,109]$ the "rebound" or recovery effect strongly depends on gate bias and with negative bias, the energy level of the oxide traps is raised considerably above the silicon conduction band edge decreasing the probability of occupancy by an electron. The $E_{o x}$ points $\uparrow$, so the weakly bound electron on the oxide trap defect can escape from the trap and tunnel back into the silicon.

Border traps and electron mobility The tenth column of Table 5.5 shows the X-ray dose dependence of $\Delta N_{o x, c h}(D)$, the charging up of border traps during the three hours after irradiation, and the eleventh column $\Delta N_{o x \text {, disch }}(D)$, the discharging of the border traps, when changing $V_{\text {gate }}$ from inversion to accumulation, remaining 3 hours in accumulation and then biasing back to inversion. The values of $\Delta N_{o x, c h}(D)$ and $\Delta N_{o x, \text { disch }}(D)$ are positive for low doses and negative for the highest doses. The absolute values are quite small compared to $N_{o x}^{e f f}$. Positive values of $\Delta N_{o x, c h}(D)$ and $\Delta N_{o x, \text { disch }}(D)$ correspond to an increase and a decrease of the $N_{o x}^{e f f}$ after 3 hours biasing in inversion and accumulation respectively. The negative values of $\Delta N_{o x, c h}(D)$ correspond to charging up of border traps with negative charge due to electron trapping in $\mathrm{Si}$, as the electric field points $\downarrow$ from the $\mathrm{SiO}_{2}$ into the $\mathrm{Si}$. Similar the negative values of $\Delta N_{o x, \text { disch }}(D)$ correspond to charging of border traps with positive charge due to hole trapping or electron de-trapping, as the electric field points $\uparrow$ from the $\mathrm{Si}$ into the $\mathrm{SiO}_{2}$. The $\Delta N_{o x, c h}(D), \Delta N_{o x, \text { disch }}(D)$ and the electric field during charging, $E_{\text {field }}^{\text {post }}$, and discharging, $E_{\text {field }}^{\text {acc }}$, are shown in Fig. 5.30.

Also the $I_{d s}\left(V_{\text {gate }}\right)$ calibrations allow to determine the dependence of the electron mobility on $V_{\text {gate }}$ for different dose values. For the parametrization, Eq. 5.2, is used. The results for $\mu_{0}$ and $V_{1 / 2}$ for the reverse calibration curves following the irradiations, are shown in Table 5.5 and in Fig. 5.31. For the non-irradiated n-MOSFET, is extracted an electron mobility at $E_{\text {field }} \approx 0$ of $\mu_{0}=1147 \mathrm{~cm}^{2} /(\mathrm{V} \cdot \mathrm{s})$, which is significantly 


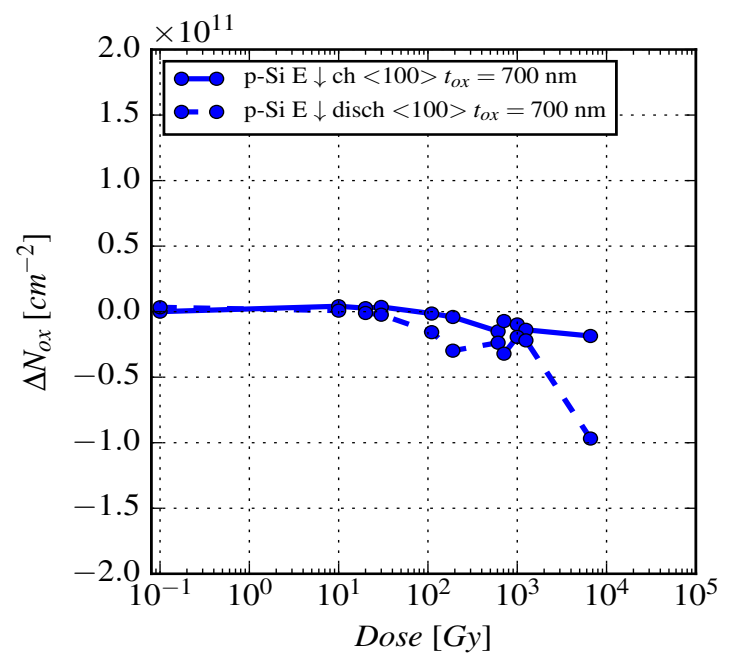

(a)

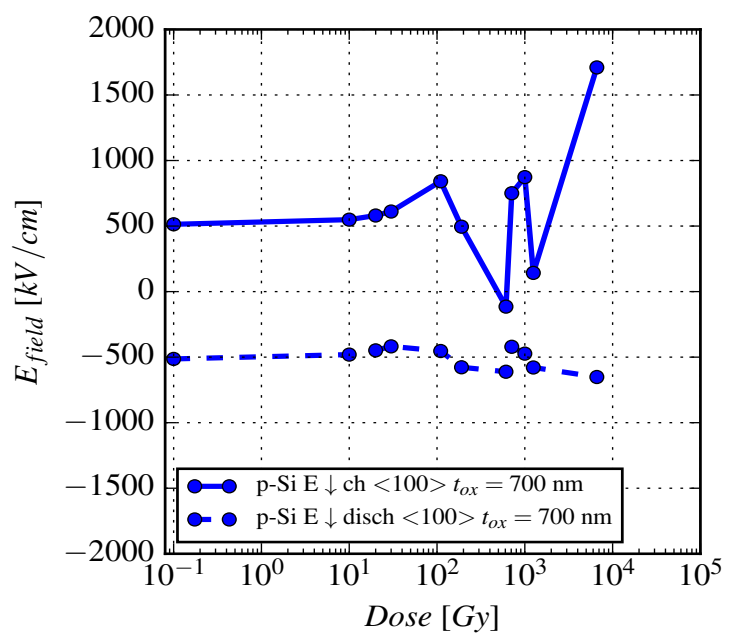

(b)

Figure 5.30: (a) $\Delta N_{o x}$ of charging and discharging of border traps, and (b) $E_{\text {field }}$ values during charging and discharging of border traps as a function of the accumulated X-ray dose of n-MOSFET irradiated with $E_{\text {field }}$ $\downarrow$.

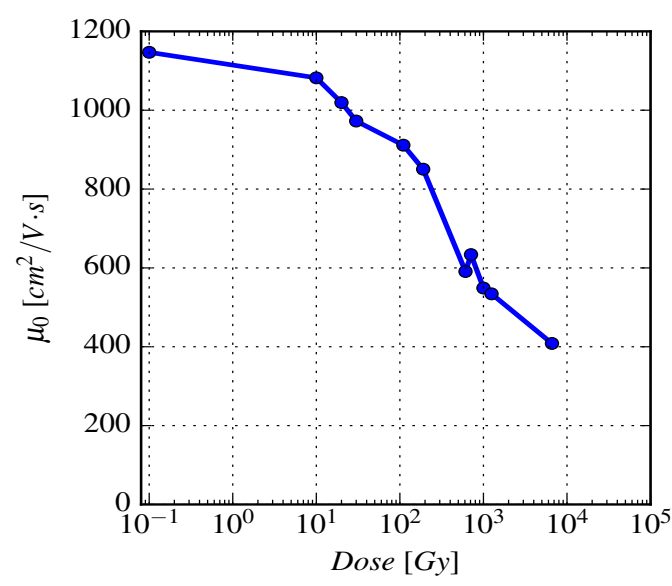

(a)

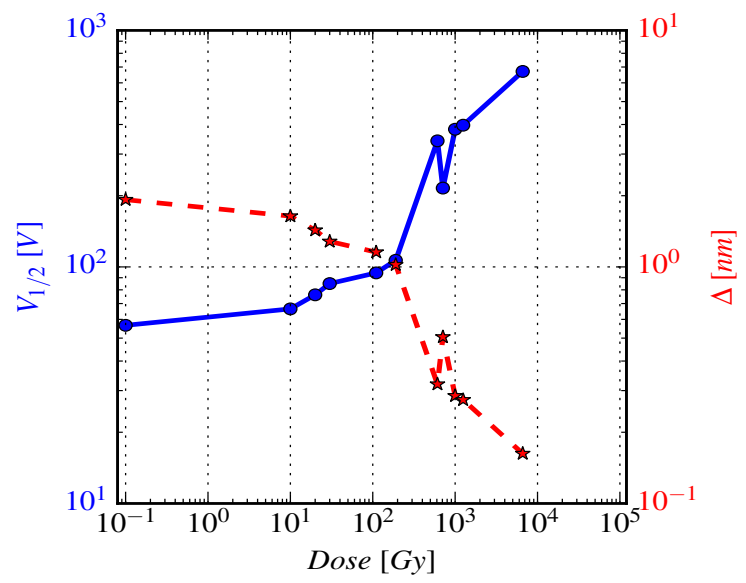

(b)

Figure 5.31: (a) Electron mobility, $\mu_{0}$, and (b) $V_{1 / 2}$ and $\Delta$ as a function of the accumulated X-ray dose of n-MOSFET irradiated with $E_{\text {field }} \downarrow$. 


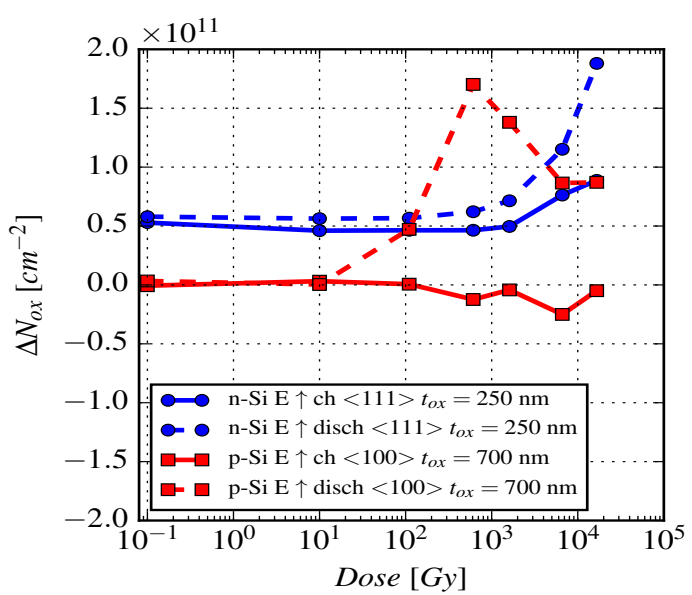

(a)

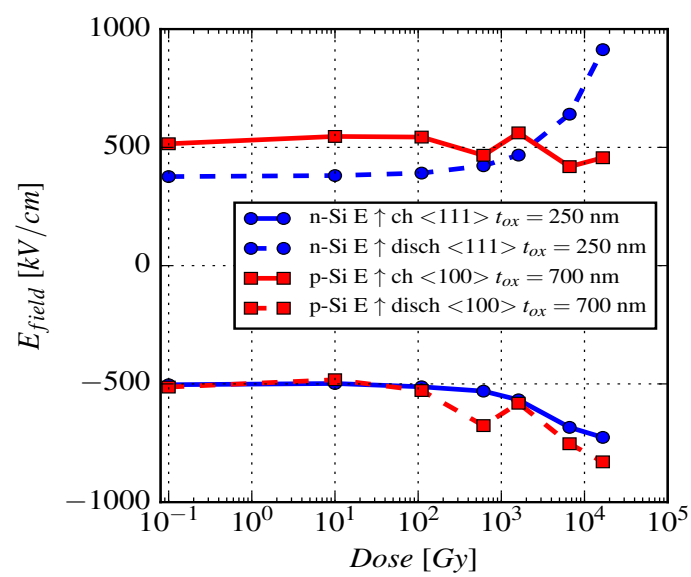

(b)

Figure 5.32: (a) $\Delta N_{o x}$ of charging and discharging of border traps, and (b) $E_{\text {field }}$ values during charging and discharging of border traps as a function of the accumulated X-ray dose of n- and p-Si irradiated with $E_{\text {field }}$ $\uparrow$.

smaller than the bulk electron mobility of $\approx 1450 \mathrm{~cm}^{2} /(\mathrm{V} \cdot \mathrm{s})$. As expected $\mu_{0}$ decreases with increasing dose. The value of $V_{1 / 2}$ increases with dose from $57 \mathrm{~V}$ to $670 \mathrm{~V}$. In Fig. 5.31 (b) is also presented the dose dependence of the surface roughness amplitude, $\Delta$, which is defined by Eq. 5.6 with the weighting factor $\eta$ equal to $1 / 2$ for electrons.

\subsubsection{Comparison}

A designer of silicon sensors could employ the above method to simulate MOS response in real time using detailed experimental data. As presented above at fixed dose rate estimates of $N_{o x}^{e f f}$, charging and discharging of border traps can be obtained, Figs. 5.32, 5.33, and mobility at the $\mathrm{Si}_{-} \mathrm{SiO}_{2}$ interface as a function of $E_{\text {field }}$ and X-ray dose, Figs. 5.34, 5.35.

These results can be used in device models (e.g TCAD) to simulate the effects of ionizing radiation on the response of silicon sensors. Firstly simulations should predict the effects of irradiations with $E_{\text {field }} \uparrow$ and $\downarrow$ in measured data of $\mathrm{p}$ - and n-MOSFETs. When the parameters used in simulations of the MOSFETs predict the data at the worst-case scenario, then the same parameters can be used to optimize the surface of silicon sensors.

Discussion on the surface mobility results The mobility degradation at the interface, Figs. 5.34 (a), 5.35 (a) often can be related simply to interface trap build-up in MOSFETs [85, 129, 138, 139]. Also it has been studied that fixed oxide charge can affect the mobility [140-144]. McLean and Boesch have developed a simple scattering model which shows that the effects of fixed oxide charge on the mobility decrease as the interface traps are annihilated or compensated by tunneling electrons [142]. They have suggested that at longer irradiation times, interface trap effects on the mobility should dominate over fixed oxide charge effects. However, even at very long irradiation times, Zupac et al. [140,141] have shown that two terms 


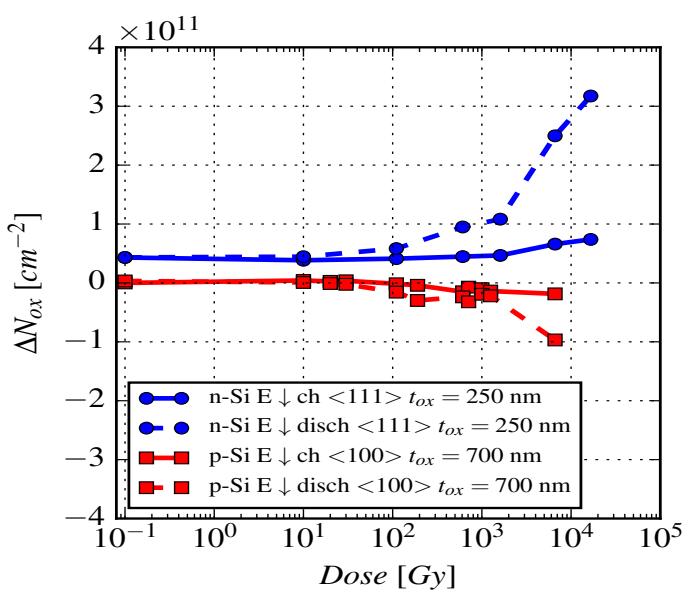

(a)

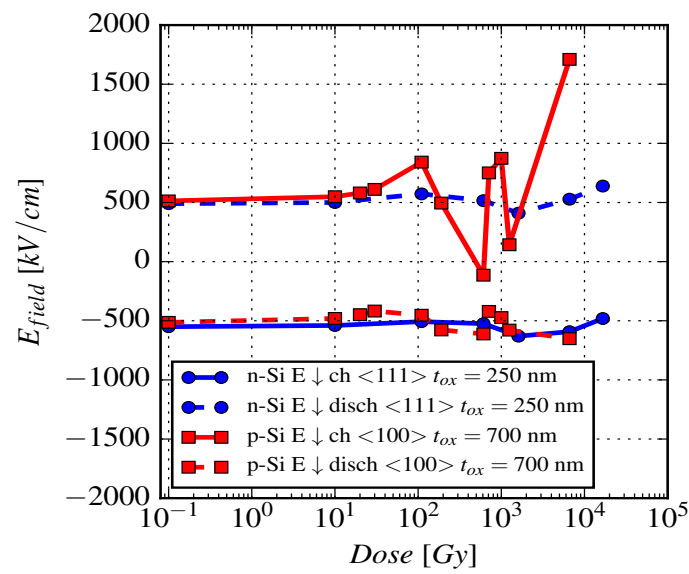

(b)

Figure 5.33: (a) $\Delta N_{o x}$ of charging and discharging of border traps, and (b) $E_{\text {field }}$ values during charging and discharging of border traps as a function of the accumulated X-ray dose of n- and p-Si irradiated with $E_{\text {field }}$ $\downarrow$.

describe the mobility degradation for MOSFET's: one related to interface traps and the second to fixed oxide charge. In particular Eq. 5.10,

$$
\mu=\frac{\mu_{0}}{1+a_{i t} \Delta N_{i t}+a_{o x} \Delta N_{o x}}
$$

where $\mu$ and $\mu_{0}$ are the post- and pre-irradiation mobilities, and $\alpha_{i t}$ and $\alpha_{o x}$ are constants related to effects of interface traps and oxide charge. According to Fleetwood et al. [129] border trap effects have not been included in Eq. 5.10. For p-MOSFETs border traps may be neutral or positively charged at inversion and

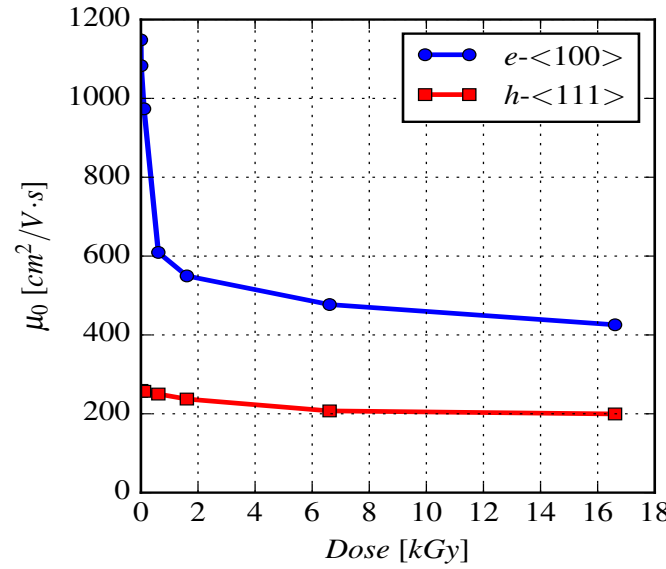

(a)

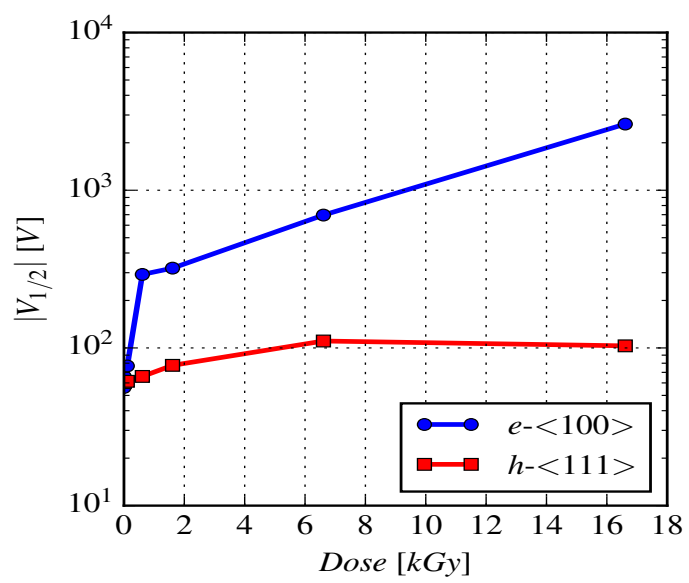

(b)

Figure 5.34: (a) Electron and hole mobility, $\mu_{0}$, and (b) $V_{1 / 2}$ as a function of the accumulated X-ray dose of n- and p-MOSFET irradiated with $E_{\text {field }} \uparrow$. 


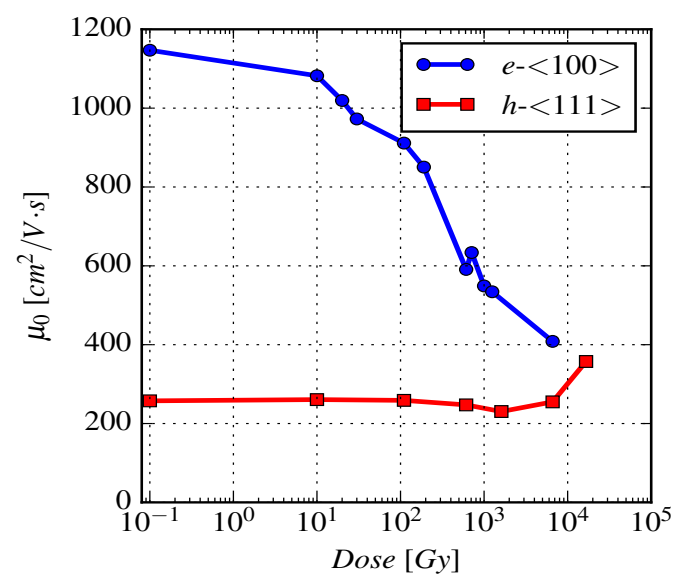

(a)

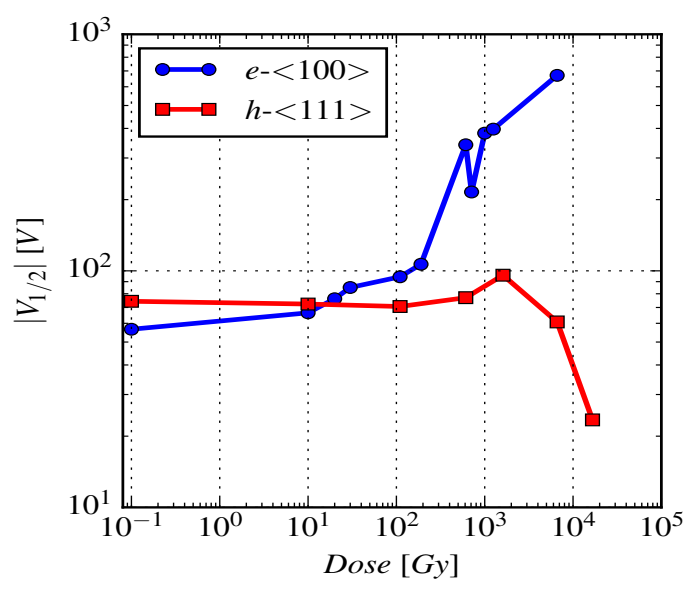

(b)

Figure 5.35: (a) Electron and hole mobility, $\mu_{0}$, and (b) $V_{1 / 2}$ as a function of the accumulated X-ray dose of n- and p-MOSFET irradiated with $E_{\text {field } \downarrow}$.

for n-MOSFETs may be neutral or negatively charged. Border traps may affect n- and p-MOSFETs under different way depending on the charge states in the two types of devices. Scattering by surface roughness due to the deviation of the interface from an ideal plane has been suggested as a possible mechanism to account for the mobility drop at high surface fields. As the surface field increases the carrier concentration in the inversion layer also increases, and the average distance of carriers from the $\mathrm{Si}_{-}-\mathrm{SiO}_{2}$ interface decreases as the inversion carriers are drawn closer to the surface. The mobility decrease at high field could possibly be due to the scattering of carriers by the fluctuating potential [145-148] resulting from the random distribution of surface charges [49]. However the mean free path of carriers and the average charge separation suggest that the fluctuating potential due to a random charge distribution cannot be the dominant mechanism responsible for the mobility decrease at high field. The surface scattering mechanism associated with the surface inhomogeneities of the $\mathrm{Si}_{-} \mathrm{SiO}_{2}$ interface. The crystal structures and the energy band pictures of $\mathrm{Si}$ and $\mathrm{SiO}_{2}$ differ, so it is not unlikely that the interface between them differs from an ideal plane. The extent of this deviation will definitely depend on the oxide structure, which in turn, is determined by both the growth conditions and subsequent annealing treatments. The physical origin of this scattering process is very simple. At any distance in the silicon from the $\mathrm{Si}_{-} \mathrm{SiO}_{2}$ interface, a charge carrier will experience a unique electric potential as a result of the applied voltage on the gate electrode. However, as the carrier moves along this plane, the electric potential is not necessarily the same as before, because the interface is not perfect. The distance of the carrier from the oxide is no longer a constant and the electric potential being a unique function of this distance, will fluctuate accordingly. A very small deviation from an ideal plane in the $\mathrm{Si}_{-} \mathrm{SiO}_{2}$ interface (in the scale of a few atomic layers) is already sufficient to result in significant scattering which causes the mobility to drop because charge carriers are drifting within a very short distance, of the order of $50 \AA$, from the surface. This distance is approximately the surface roughness amplitude, $\Delta$, which is calculated above for $\mathrm{p}$ - and n-MOSFETs. As the surface field increases, carriers are drawn closer to the surface, consequently the fluctuating potential becomes much stronger. The relaxation time due to this mechanism then dominates over that due to Coulomb scattering. The mobility values at high field depend on sample preparation and become independent of the oxide charge. Nevertheless, as presented above the dose 
dependence of $V_{1 / 2}$ and $\Delta$ indicates that the surface field is influenced by the increase of the oxide charge and interface traps. Scattering by surface roughness will always be present in any surface channel or layer, because of the misfit of the lattice spacings between $\mathrm{Si}$ and $\mathrm{SiO}_{2}$ and the presence of interface stress due to the difference in thermal expansion coefficient, therefore always the interface is expected to deviate slightly from a perfect plane.

Discussion on the border traps results In the literature a revised nomenclature for defects in MOS devices has been developed that clearly distinguishes the terms used to describe the physical location of defects from that used to describe their electrical response. According to this nomenclature, oxide traps are defects in the $\mathrm{SiO}_{2}$ layer of the MOS structure, and interface traps are defects at the $\mathrm{Si}_{-} \mathrm{SiO}_{2}$ interface. Electrically, "fixed states" are defined as trap levels that do not communicate with the Si on the time scale of the measurements, but "switching states" can exchange charge with the Si. Fixed states are considered as oxide traps, but switching states can either be interface traps or near interfacial oxide traps that can communicate with the Si, defined as "border traps" [132]. The effective density of border traps depends on the time scale and bias conditions of the measurements. Border trap densities of the order of $10^{10}-10^{11} \mathrm{~cm}^{-2}$ as presented above, are inferred from changes in switching state density during post-irradiation biasing. Trapped-hole, trapped-electron and switching state densities are inferred via Thermally Stimulated Current TSC [149-151], Capacitance-Voltage C-V [152-156] and Current-Voltage I-V measurements.
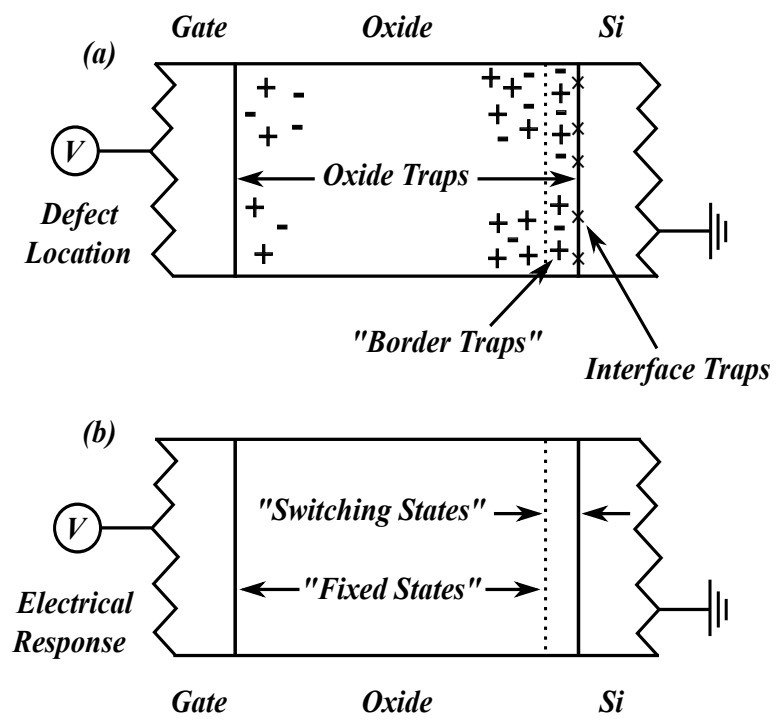

Figure 5.36: Diagram that illustrates a revised defect nomenclature for MOS devices which distinguishes terms used to describe (a) the physical location of defects (b) from their electrical response. Figures adapted from [132].

In particular border traps can act either as bulk oxide traps or interface states as shown in Fig. 5.36 (a). The line between a border trap and an oxide trap is determined by the proximity of the defect to the $\mathrm{Si}-\mathrm{SiO}_{2}$ interface and the energy of the trap. A clear separation between the electrical response and the physical structure and location of defects in the $\mathrm{Si}_{-} \mathrm{SiO}_{2}$ system is not always successful. For example, the interface states in one study may be $P_{b}$ defects located at the $\mathrm{Si}_{-} \mathrm{SiO}_{2}$ interface (interface traps), but in another study they may be $E^{\prime}$ centers $[96,157,158]$ located in the oxide within $0.2-3 \mathrm{~nm}$ of the $\mathrm{Si}_{-} \mathrm{SiO}_{2}$ interface (border traps). Based only on electrical data, it is often not possible to distinguish one type of physical defect from 
another. In Fig. 5.36 (b) are defined as "fixed states" those defects that do not exchange charge with the $\mathrm{Si}$ on the time scale of the measurements, and "switching states" those defects that do exchange charge with the $\mathrm{Si}$. Border traps are those near interfacial oxide traps that function electrically as switching states on the time scale of the measurements. The number of oxide traps that function as switching states can be a strong function of the time scale and bias conditions of the measurements. Three types of centers consistent with the properties of border traps were identified in the literature, Fig. 5.37 (a).

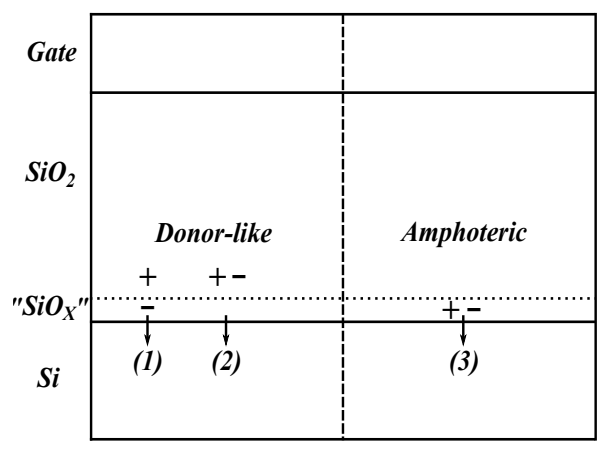

(a)

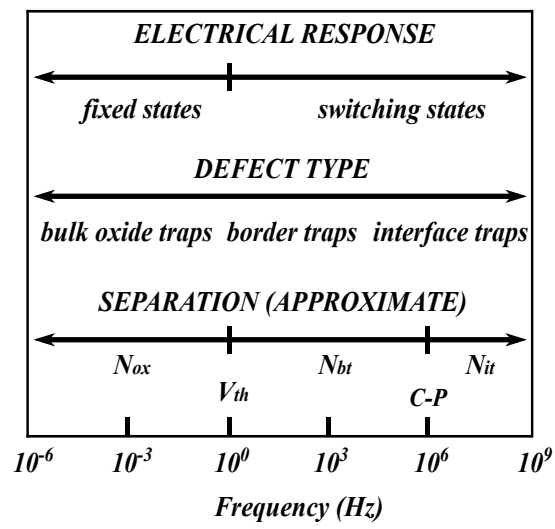

(b)

Figure 5.37: (a) Three possible physical pictures of border traps in irradiated MOS devices: a donor-like trap (type 1), a deep donor-like trap (type 2), and an amphoteric border trap (type 3), (b) Illustration of communication rates with the underlying $\mathrm{Si}$ for bulk oxide traps, border traps, and interface traps. Figures adapted from [132].

Types (1) and (2) are donor-like centers associated with trapped positive charge in the oxide which can exchange an electron with the Si. This sort of defect structure has been inferred from annealing and TSC measurements of MOS devices. In a type (1) donor trap, the electron is pictured at a metastable side (associated with the trapped hole) in the transitional region of the oxide, $\mathrm{SiO}_{x}$, which may not be stoichiometric $\mathrm{SiO}_{2}$. Such a site is consistent with the observation that trapped electrons are not present in significant densities in irradiated $\mathrm{SiO}_{2}$ in the absence of trapped holes, but can be present in large densities when holes are trapped in the $\mathrm{SiO}_{2}$. Type (2) is a deeper donor-like trap in the bulk of the $\mathrm{SiO}_{2}$ layer, and the electron may even be trapped at the physical defect site, for example the $E^{\prime}$ center as the trapped hole [159]. The type (1) trap, where the electron is closer to the $\mathrm{Si}-\mathrm{SiO}_{2}$ interface, probably has a lower barrier to the electron injection and can communicate more rapidly with the Si than type (2). As result type (1) appears to be a more likely candidate for a border trap in irradiated MOS devices. Type (2) may play an important role in the long-term "reversibility" of trapped positive charge neutralization in MOS devices. Type (1) and (2) may be similar to the (donor-like) "anomalous positive charge" observed in studies of oxides exposed to high field stress. Type (3) is a different type of defect, in which both the associated hole and electron may be exchanged with the $\mathrm{Si}$, depending on the bias conditions at the $\mathrm{Si}_{-} \mathrm{SiO}_{2}$ interface. Fig. 5.37 (b) presents the ranges of communication times of bulk oxide traps, border traps, and interface traps with the underlying $\mathrm{Si}$. Threshold voltage measurements as described above, are performed at an effective frequency of $\approx 1 \mathrm{~Hz}$, traps which exchange charge with the underlying $\mathrm{Si}$ on time scales less than $\approx 1 \mathrm{~s}$ will function electrically 
as "fixed states", while faster traps will switch charge states during an I-V measurement performed to estimate the threshold voltage. So far measurements at a single effective frequency cannot easily discriminate between the effects of interface traps and border traps (faster than the characteristic measurement time) on the device response. Charge pumping measurements [160] for example at a frequency of $\approx 1 \mathrm{MHz}$ allow an appropriate separation of bulk oxide trap, border trap, and interface trap effects. In particular traps that exchange charge with the $\mathrm{Si}$ at frequencies less than $\approx 1 \mathrm{~Hz}$ are classified as oxide traps, traps that exchanging charge at $\approx 1 \mathrm{MHz}$ or below are classified as border traps. Some border traps will exchange charge with the $\mathrm{Si}$ on time scales slower than $\approx 1 \mathrm{~s}$, while some others (very near the interface) may communicate with the $\mathrm{Si}$ on time scales faster than $\approx 10^{-6} \mathrm{~s}$. In conclusion the above analysis illustrates the charge state of the border traps under device operating conditions. For n-MOSFETs, border traps may either be neutral or negatively charged near inversion. Thus, border traps affect $n$ - and p-MOSFETs differently, depending on their respective charge states in the two types of devices.

\subsection{Summary}

In this chapter a measurement cycle is developed, which allows to determine the radiation-induced effects on oxide charge, the charging and discharging of border traps and the change of the surface mobility with irradiation. It has been applied on p- and n-MOSFETs with electric field pointing into the $\mathrm{SiO}_{2}$ and into the Si during irradiation. For the n-MOSFET with the electric field pointing into the Si and the p-MOSFET pointing into the $\mathrm{SiO}_{2}$ the changes of the effective oxide charges during irradiation can be monitored. X-ray irradiation in different steps up to a total dose of $16.61 \mathrm{kGy}$ have been performed. It has been found that no particular effects happen during or shortly after the irradiation. The effective oxide charge density is a smooth function of time during the irradiation cycle. As expected, the radiation-induced oxide charge density depends on the electric field and the type of charge carriers, electrons or holes at the $\mathrm{Si}_{-} \mathrm{SiO}_{2}$ interface, during irradiation. For the p-MOSFET the effective oxide charge density increases to $2.0 \cdot 10^{12}$ and $1.3 \cdot 10^{12} \mathrm{~cm}^{-2}$ after a dose of $16.61 \mathrm{kGy}$ for the electric field pointing into the $\mathrm{SiO}_{2}$ and into the $\mathrm{Si}$, respectively. For the latter case, a value of $10^{12} \mathrm{~cm}^{-2}$ is reached already at around $1 \mathrm{kGy}$, beyond which it increases only slowly. For the n-MOSFET for both field directions the effective oxide charge first increases with irradiation. However for higher dose values the effect of charge neutralization sets in, resulting in a reduction of the effective oxide charges and even negative values. These observations can be described by the neutralization and rebound effects discussed in the literature. For the p-type $\mathrm{Si}$, the acceptor-type interface traps in the upper half of the silicon bandgap below the Fermi level will be negatively charged. The donor-type interface traps in the lower half of the bandgap will be neutral. Electrons can tunnel either from the oxide valence band or from the silicon and neutralize the oxide charge density. Time and temperature dependence of rebound can be accounted by a thermally assisted tunneling process. The p-MOSFETs do not show rebound effects after irradiation. The above observations about radiation-induced charge neutralization and rebound effects indicate evidence for space charge effects in the $\mathrm{SiO}_{2}$ which can influence the electric field in a sensor and finally the breakdown voltage. 


\section{Subthreshold current technique}

In this chapter is presented the subthreshold current technique. The development of the technique is based on Refs. $[102,103,161]$ and is applied on high-ohmic $n$ - and p-MOSFETs designed on the same wafer with silicon sensors.

\subsection{Introduction}

Silicon sensors which use $\mathrm{SiO}_{2}$ for the gate dielectric and surface passivation change their response when exposed to ionizing radiation. The change results from two phenomena, 1) radiation-induced oxide charge, $\Delta N_{o x}$ and 2) radiation-induced interface traps $\Delta N_{i t}$. Ionization induced effects on transistors are the result of electron-hole pair generation and recombination in $\mathrm{SiO}_{2}$, hole transport and trapping, interface trap formation mechanisms, the chemical structure of the hole trap and interface trap, the energy distribution density $D_{i t}$ of the interface traps, the de-trapping mechanisms of trapped holes and the annihilation mechanisms for interface traps. Many models have been developed during the last 50 years for the observed characteristics and their dependence on dose, dose rate, electric field, temperature, oxide thickness and oxide processing. Most of the research to characterize the radiation-induced trapped hole and interface trap densities has been done with a nearly ideal, one dimensional test structure, the MOS Capacitor, MOSC. The primary techniques used to study the mechanisms of $\Delta N_{o x}$ and $\Delta N_{i t}$ on capacitors involve capacitance-voltage or conductance-voltage measurements. These measurements are made as function of temperatures, sweep rates and frequency to characterize the trapping and de-trapping of $\Delta N_{o x}$ and the energy dependence of $\Delta N_{i t}$ in the Si bandgap. Other techniques involving charge injection and gate current measurements are used to measure the spatial dependence and energy levels of the trapped holes. Although the MOSC is an ideal structure for studying the physics of $\Delta N_{o x}$ and of $\Delta N_{i t}$, however there are practical limitations for applications such as device characterization and hardness assurance [162]. For example 1) MOSCs are not used to build silicon sensors, 2) practical bias conditions cannot be evaluated (eg. pn junction bias) and 3) geometrical effects cannot be evaluated. Several failure mechanisms relating to interface traps started to dominate such as, mobility degradation and positive threshold voltage shifts in n-channel MOSFETs referred to as "rebound" or recovery [106]. It has long been recognized in the microelectronics and space radiation community that the most practical test structure for characterizing the ionization response of the MOS system is the MOSFET [163]. The subthreshold technique, which was first presented in 1984 [102] and was formalized in 1986 [103], is based on standard I-V characteristics and is the only technique which combines a method for $\Delta V_{o x}$ and $\Delta V_{i t}$ determination. The subthreshold drain-source current $I_{d s}$ at a fixed drain to source voltage, $V_{d s}$, is measured as a function of gate voltage $V_{\text {gate }}$. In an ideal device, the $I_{d s}$ and $V_{\text {gate }}$ are related by $I_{d s} \propto \exp \left(V_{\text {gate }}\right)$. 
When $I_{d s}$ is plotted as a function of $V_{g a t e}$ in log scale, the straight I-V characteristic can be extrapolated to a calculated midgap current, Fig. 6.1.

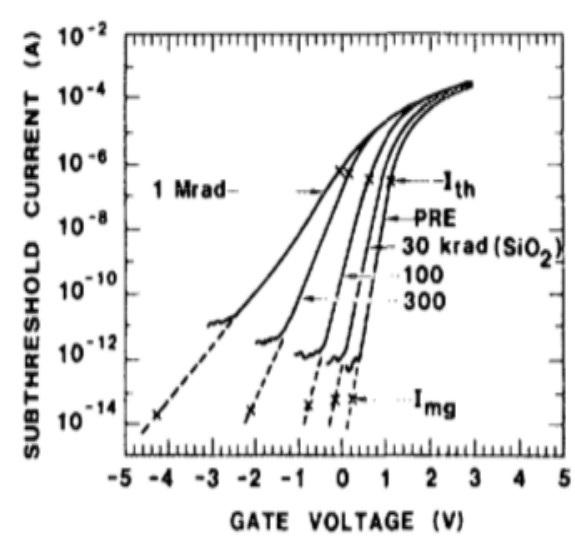

(a)

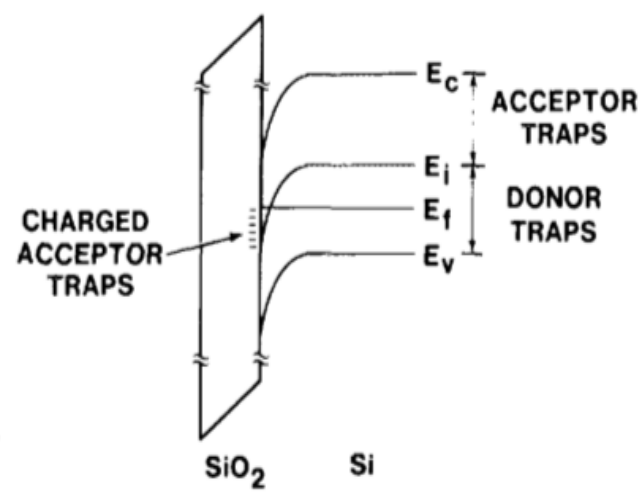

(b)

Figure 6.1: (a) Subthreshold-current curves for an n-MOSFET before and after ${ }^{60} \mathrm{Co}$ irradiation. Threshold and midgap currents are marked on each curve. (b) Energy band diagram of an n-MOSFET in strong inversion showing charged acceptor traps. Figures adapted from [102].

Comparing the pre- and post-irradiation characteristics, the midgap voltage shift, $\Delta V_{m g}$, as well as the change in subthreshold swing (inverse slope), $\Delta S$ can be determined. The value of $\Delta V_{m g}$ corresponds to $\Delta V_{o x}$ and $\Delta S$ is proportional to $\Delta V_{i t}$.

\subsection{MOSFET subthreshold theory}

MOSFET subthreshold theory refers to the electrical characteristics of the transistor in weak inversion. In weak inversion, the current in the channel is dominated by diffusion of minority carriers. This differs from the strong inversion channel current which is dominated by electric field assisted majority carrier transport. The subthreshold current is used to determine the shift in threshold voltage due to trapped charges, $\Delta V_{o x}$, and $\Delta V_{i t}$. The accuracy of this calculation is dependent on whether assumptions used to develop the subthreshold charge separation technique are violated. In this section, the MOSFET subthreshold theory is presented. In the next sections, the validity of the assumptions are checked in order to determine their influence on the subthreshold charge separation results.

\subsubsection{Theoretical MOSFET weak inversion channel current}

In a MOSFET, the subthreshold channel current, $I_{d s}$, is given by

$$
I_{d s}=\mu\left(\frac{W}{L}\right)\left(\frac{n_{i}}{N_{d}}\right)^{2} \frac{\alpha C_{o x}}{2 \beta^{2}} e^{\beta \phi_{s}}\left(\beta \phi_{s}-1\right)^{-1 / 2}
$$


where $\mu$ is the effective channel carrier mobility, $N_{d}$ the interface doping, $n_{i}$ the intrinsic doping concentration, $W$ the channel width, $L$ the channel length, $\beta=q_{0} / k T, \phi_{S}$ the surface potential, $\alpha=\sqrt{2}\left(\varepsilon_{S i} / L_{D}\right) / C_{o x}$, $\varepsilon_{S i}$ the silicon dielectric constant, $L_{D}$ the Debye length, and drain voltage is a few $k T / q_{0}$. This equation is derived using the Brews charge sheet model $[28,36,164]$. The current in this case refers to the current which flows in the channel of the device. It does not include gate and substrate leakages or any other parasitic effect. The channel current in Eq. 6.1 is a strong function of the surface potential. The gate voltage is related to the surface potential so that the $\frac{d V_{\text {gate }}}{d \ln I_{d s}}$ can be calculated. This derivative gives the gate voltage swing needed to reduce the current by one decade, and is called the subthreshold swing. The subthreshold swing, $\mathrm{S}$, is defined by

$$
S=\beta^{-1} \ln 10 \frac{d \beta V_{\text {gate }}}{d \beta \phi_{s}} \frac{d \beta \phi_{s}}{d \ln I_{d s}}
$$

Changes in the variables, which determine the channel current, can affect the subthreshold swing, if they are a function of the surface potential. Eq. 6.2 is used to determine the midgap current in the subthreshold technique. To calculate the derivative in Eq. 6.2, a function relating the gate voltage to the surface potential is needed. Using the depletion approximation, the following equation was derived by Brews [164]

$$
\beta V_{\text {gate }}=\beta \phi_{s}+a\left(\beta \phi_{s}-1\right)^{1 / 2}
$$

Using Eqs. 6.2 and 6.3, and assuming that all coefficients do not change with surface potential, the derivative in Eq. 6.2 can be calculated and the subthreshold swing becomes

$$
S=\beta^{-1} \ln 10 \frac{1+\frac{C_{d e p}}{C_{o x}}}{1-\frac{2}{\alpha^{2}}\left(\frac{C_{d e p}}{C_{o x}}\right)^{2}}
$$

where $C_{d e p}$ is the channel depletion capacitance. The subthreshold swing is not expected to be constant from midgap to inversion due to the depletion capacitance. The depletion capacitance results from the depletion of majority carriers in the channel region and is in series with the insulator (gate) capacitance. As the surface potential is increased, the depletion region grows and the total capacitance decreases. The minimum total capacitance occurs between midgap and inversion and this is also the point at which the minimum subthreshold swing occurs. The swing increases as the surface potential is increased or decreased.

\subsubsection{Subthreshold charge separation technique}

There are two methods to separate the threshold voltage into a voltage shift due to oxide charge, $\Delta V_{o x}$ and interface traps $\Delta V_{i t}$ using the subthreshold technique. The two methods depend on whether $\Delta V_{o x}$ or $\Delta V_{i t}$ is directly calculated from the subthreshold curve and are referred to as, 1) Midgap voltage method and 2) Subthreshold swing method.

Threshold voltage $V_{t h}$ : The parameter most often used to characterize the radiation hardness of a MOSFET is the threshold voltage $V_{t h} . \Delta V_{t h}$ is given by 


$$
\Delta V_{t h}=V_{t h}^{p o s t}-V_{t h}^{p r e}
$$

where $V_{t h}^{\text {pre }}$ the pre-irradiation and $V_{t h}^{\text {post }}$ the post-irradiation threshold voltage.

\section{Midgap voltage method}

The midgap voltage method of the subthreshold charge separation technique is based on two quantities: 1) The radiation-induced voltage shift from the oxide charge, $\Delta V_{o x}$, determined by the change in midgap voltage, $\Delta V_{m g}$, and 2) The radiation-induced voltage shift from interface states, $\Delta V_{i t}$, using $\Delta V_{m g}$ and the change in the threshold voltage, $\Delta V_{t h}$. The value of $\Delta V_{o x}$ is obtained from $\Delta V_{m g}$ assuming the following relation

$$
\Delta V_{o x}=\Delta V_{m g}=\frac{q_{0} \Delta N_{o x}}{C_{o x}}
$$

and $\Delta N_{o x}$ is the radiation-induced oxide charge density. Eq. 6.6 is based on the assumptions that the oxide charge density is a uniform sheet charge at the interface and the net charge of the interface traps at midgap is zero [96].

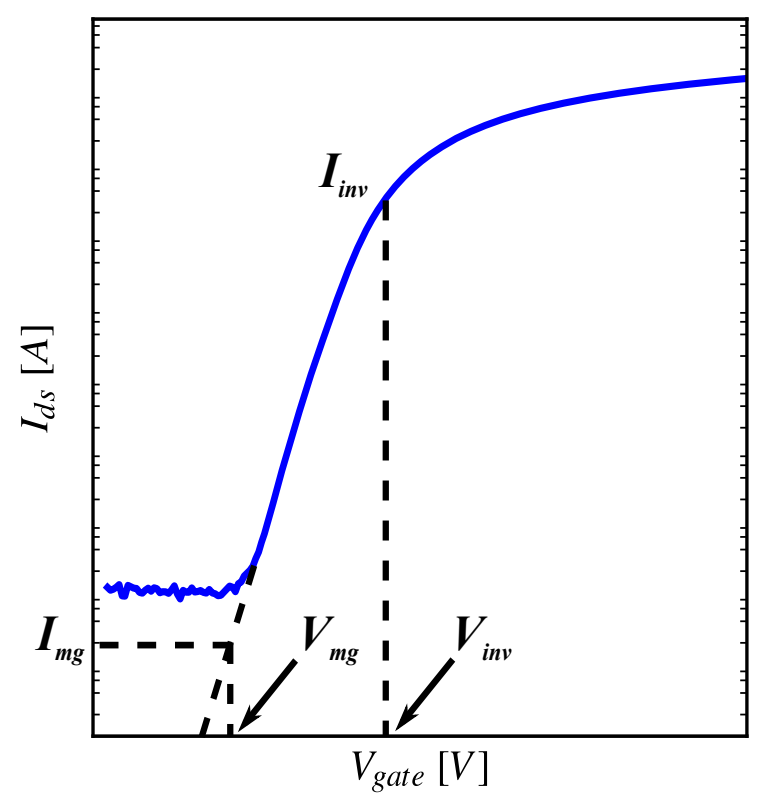

Figure 6.2: Typical n-MOSFET of $I_{d s}$ as a function of $V_{\text {gate }}$ in $\log$ scale showing the extrapolation to the calculated midgap current.

In the application of the subthreshold charge separation technique, the radiation-induced interface traps are assumed to be primarily acceptors in the upper half of the bandgap and donors in the lower half. Near midgap, the net charge of the interface states is approximately zero. If the net charge of the interface traps at midgap is zero, then the change in midgap voltage is a result of the net oxide trapped charge which is assumed to be holes. The midgap voltage usually cannot be measured directly because the channel current 
at midgap is usually small to be measured with conventional picoammeters or electrometers. Therefore, an extrapolation technique is used to determine the midgap voltage from the channel current, $I_{d s}$, versus the gate voltage, $V_{\text {gate }}$, in the subthreshold region. The channel current in subthreshold (weak inversion) is given in Eq. 6.1. The channel current at midgap, $I_{m g}$, can be found by substituting the midgap value of surface potential, $\phi_{b}$, into Eq. 6.1. The midgap voltage, $V_{m g}$ is the gate voltage at $I_{m g}$ found by extrapolating the measured subthreshold characteristic. In Fig. 6.2 is presented the $I_{d s}$ as a function of $V_{g a t e}$ in $\log$ scale.

$$
\phi_{s}=\phi_{b}=\frac{k T}{q_{0}} \ln \left(\frac{N_{d}}{n_{i}}\right)
$$

The radiation-induced voltage shift due to charged interface traps is assumed to be that portion of the inversion or threshold voltage shift which is not due to the trapped oxide charge. The use of inversion voltage is mathematically more correct since it is the value of gate voltage for a specified value of the surface potential. However, the use of the threshold voltage, which is an empirical value, is more practical since it is the parameter specified on measured data and generally used to measure radiation performance. The threshold voltage change due to irradiation, $\Delta V_{t h}$, is a result of the voltage shift due to trapped oxide charge, $\Delta V_{o x}$, and the voltage shift due to the charged interface states, $\Delta V_{i t}$. In n-channel devices, the value of $\Delta V_{i t}$ is positive due to the predominance of acceptors, and in p-channel devices, $\Delta V_{i t}$ is negative due to the predominance of donors. Using the midgap voltage method, the value of $\Delta V_{i t}$ is found from Eq. 6.8

$$
\Delta V_{i t}=\Delta V_{t h}-\Delta V_{o x}
$$

and $\Delta N_{i t}$ can be found from Eq. 6.9

$$
\Delta N_{i t}=\frac{C_{o x} \Delta V_{i t}}{q_{0}}
$$

\section{Subthreshold swing method}

The subthreshold swing method of the charge separation technique is based on two calculations: 1) The radiation-induced voltage shift due to interface traps, $\Delta V_{i t}$, using the change in subthreshold swing, $\Delta S$, and 2) The radiation-induced shift from oxide charge, $\Delta V_{o x}$, using $\Delta V_{i t}$, and the change in threshold voltage, $\Delta V_{t h}$ Assuming $\alpha>>C_{d e p} / C_{o x}$, the pre-irradiation subthreshold swing $S^{p r e}$ can be written as

$$
S^{p r e}=\frac{k T}{q_{0}} \ln 10\left(1+\frac{C_{d e p}}{C_{o x}}\right)
$$

and the post-irradiation subthreshold swing, $S^{\text {post }}$, is defined by equation,

$$
S^{p o s t}=\frac{k T}{q_{0}} \ln 10\left(1+\frac{C_{d e p}+C_{i t}}{C_{o x}}\right)
$$


The difference between the pre- and post-irradiation subthreshold swings, $\Delta S$, is

$$
\Delta S=S^{p o s t}-S^{p r e}=\frac{k T}{q_{0}} \ln 10 \frac{C_{i t}}{C_{o x}}
$$

If the interface states are uniformly distributed between midgap and inversion, the interface state capacitance, $C_{i t}$, causes the voltage shift, $\Delta V_{i t}$, which is equivalent to

$$
\Delta V_{i t}=\frac{C_{i t}}{C_{o x}} \phi_{b}
$$

Using Eq. 6.12 and 6.13, the value of $\Delta V_{i t}$ is obtained from $\Delta S$ where

$$
\Delta V_{i t}=\frac{q_{0} \phi_{b}}{k T \ln 10} \Delta S
$$

Eq. 6.14 is based on the following assumptions, 1) no lateral nonuniformities of trapped oxide charge in the oxide, 2) the net charge of interface traps at midgap is zero and is uniform between midgap and inversion and 3) $\alpha>>C_{d e p} / C_{o x}$ and $\alpha>>\left(C_{d e p}+C_{i t}\right) / C_{o x}$. The assumption that $\alpha>>\left(C_{d e p}+C_{i t}\right) / C_{o x}$ is a fairly stringent restraint. As the number of interface traps increases, $C_{i t}$ also increases. This assumption essentially limits the application of this method. At high total dose levels, this assumption should be checked. Similar to the midgap voltage method, the voltage shift due to oxide charge, $\Delta V_{o x}$, is calculated using the shift in threshold or inversion voltage from the

$$
\Delta V_{o x}=\Delta V_{t h}-\Delta V_{i t}
$$

\subsection{Discussion of variables and assumptions in the subthreshold charge sep- aration technique}

The analysis of a MOSFET that has a near linear subthreshold swing using the subthreshold charge separation technique is well defined. The subthreshold swing is extrapolated to find the midgap voltage. The extrapolation is necessary because the midgap current is below the leakage of the device (for low-ohmic Si substrate) and the instrumentation is not capable of measuring such low current levels. When a device exhibits a nonlinear subthreshold swing, one must choose what part of the curve to extrapolate. In some cases, the choice can yield widely different midgap voltages, which affects the trapped hole and interface state results. The correct choice depends on the purpose of the analysis and on the robustness of the subthreshold charge separation technique. There are inherent assumptions in the theoretical development, and violations of these assumptions may or may not greatly affect the analysis results. To analyze nonlinear characteristics, the analyst must hypothesize possible causes and determine how the subthreshold technique is affected. Combining these hypotheses with the aim of the study, the analyst must determine the portion of the curve to extrapolate [161]. 


\subsubsection{Mobility}

Mobility is an important parameter in equation. Two mobility regions can be defined in a MOSFET, 1) the mobility which occurs in strong inversion and 2) the mobility in weak inversion or subthreshold. The strong inversion mobility is greatly affected by the surface of the $\mathrm{Si}_{-} \mathrm{SiO}_{2}$ interface. In the inversion region, the vertical electric field is much greater than the transverse electric field. This pulls the carriers to the interface, lowering the mobility due to the roughness of the $\mathrm{Si}_{-} \mathrm{SiO}_{2}$ interface. The rougher the interface the lower the mobility. In the subthreshold region, the transverse electric field is smaller, and the interface roughness has less effect on the mobility. Thus, as the surface potential is lowered from strong to weak inversion, the interface roughness has less effect and the mobility increases. The mobility in Eq. 6.1 is the weak inversion mobility and can be substantially different than the strong inversion mobility. The bulk mobility in silicon is a function of total impurity concentration (the number of charged impurity atoms). When both n- and p-type impurities exist in silicon, acceptors receive electrons from the donors and all impurity atoms are charged. Thus, in theory, the weak inversion mobility can be no larger than the bulk mobility. The value of mobility affect the midgap current and hence the determination of the midgap voltage. The channel mobility as a function of surface potential has been studied. Measurements of mobility have not been done for surface potentials much below inversion. The effect of surface potential and radiation-induced oxide charge and interface traps on channel mobility has been suggested [165]. Fig. 6.3 (a) shows a possible scenario for a typical unirradiated device.

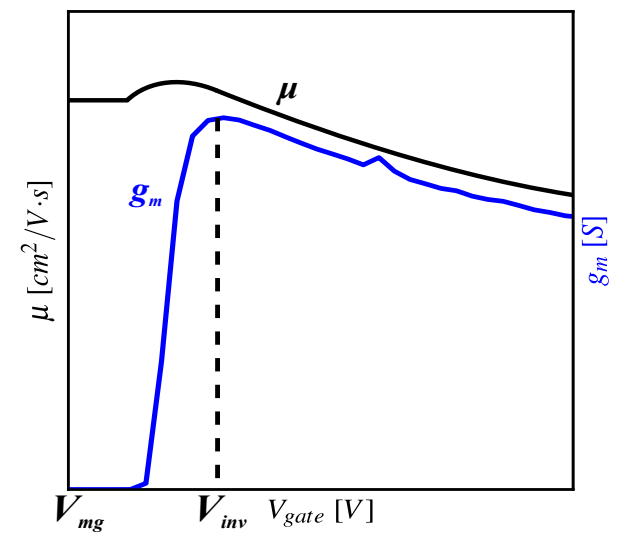

(a)

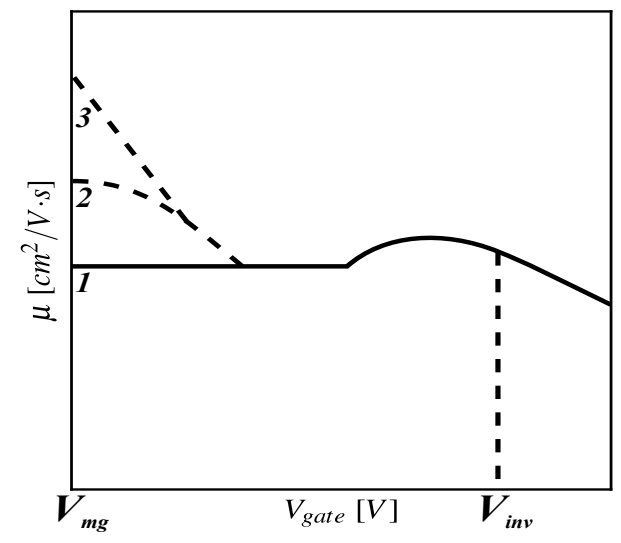

(b)

Figure 6.3: (a) Effect of increasing mobility near midgap on the extrapolation of the subthreshold curve, (b) Possible effect of oxide charges and interface traps on mobility for an irradiated MOSFET. Figures adapted from [161].

Little change is expected to occur in the subthreshold region. It has been generally accepted that ionizing radiation induces interface traps and trapped oxide charge within $5 \mathrm{~nm}$ of the surface degrade mobility. Interface traps act as charged scattering centers and degrade the mobility similar to impurity doping. Nonuniformity of interface traps and trapped charge can create further degradation in the mobility by causing minority carrier fluctuations, which have a large effect near inversion. For low trapped charge densities, the mobility is expected to decrease with surface potential above inversion Fig. 6.3 (a), but for high trapped charge densities, it can increase with surface potential because of the decreasing effect of minority carrier fluctuations. 
Fig. 6.3 (b) shows possible effects of the radiation-induced trapped charges. The curves of interest are the ones which show mobility increasing as the surface potential approaches midgap. The hypothesis for this occurrence relies on the assumption that most interface states have neutral charge at midgap. This implies the post-irradiation mobility near midgap should increase toward the pre-irradiation condition. Since studies have shown post-irradiation mobility is reduced in the subthreshold, the subsequent increase seems possible, due to the decrease in charged interface traps. The question is how rapidly does the mobility increase as the surface potential approaches midgap. The increase in mobility near inversion is caused by a decreased effect from minority fluctuations. If the mobility follows curve 3 in Fig. 6.3 (b), the effect on the subthreshold swing would be noticeable. At some point on the subthreshold curve, as the surface potential approaches midgap, the swing would increase. This could cause a second swing or increasing swing condition as shown in Fig. 6.4. The lower portion of the curve has a larger voltage swing per decade. In this case, it would be caused by a changing mobility and not additional by interface states in the bandgap. Extrapolation of measured data to find the midgap voltage requires judgement. The subthreshold curve will not continue at the larger swing, when the mobility is near the maximum value. Extrapolating from the top or bottom of the curve may not yield the correct midgap voltage. An example is given in Fig. 6.5 (a) for the effect of the increasing mobility as shown in Fig. 6.3 (b) on the subthreshold curve. To analyze this data using the subthreshold technique, one must decide from which part of the curve to do the linear extrapolation. Since the subthreshold technique assumes that the mobility is constant and uses Eq. 6.1, the first swing should be used. This will give the voltage shifts caused by the positive trapped oxide charge. If the second swing is used, the technique will give the voltage shifts caused by the number of interface traps and trapped oxide charge to produce the combined trapped charge and mobility effect on the subthreshold swing.

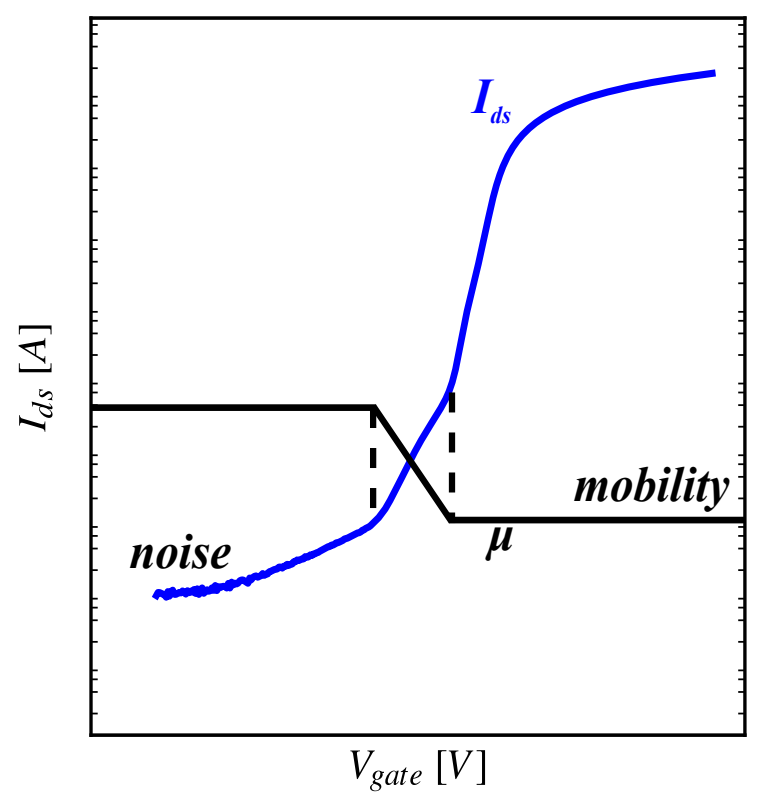

Figure 6.4: Effect of varying mobility on the MOSFET subthreshold curve.

Assuming a nonuniform mobility, the choice of which part of the curve to use in Fig. 6.5 (b) to extrapolate depends on the purpose of the analysis. To find the correct number of trapped holes and interface states in the gate oxide, the top part of the curve is used. 


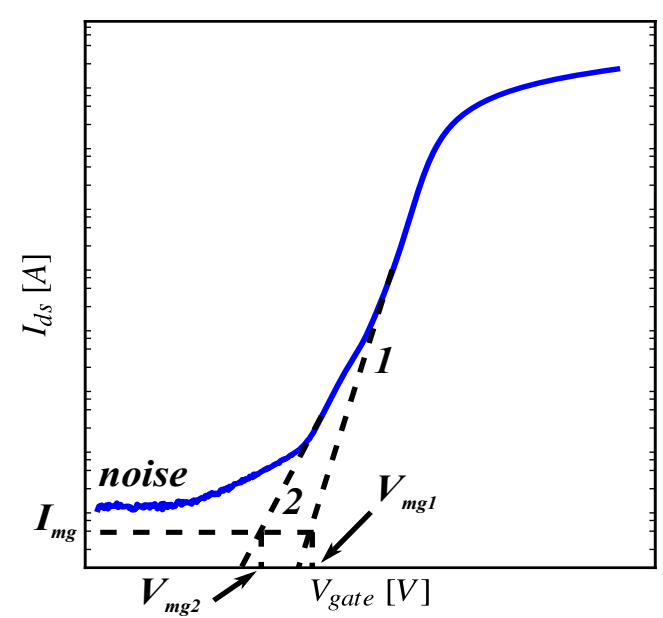

(a)

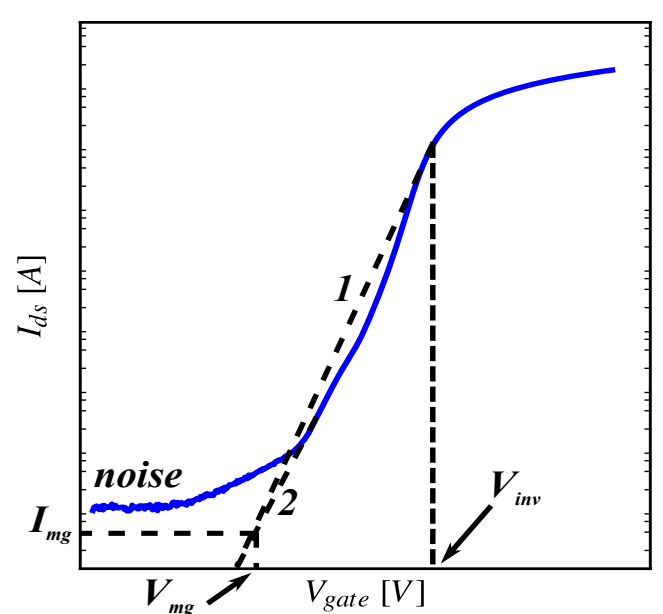

(b)

Figure 6.5: (a) Effect of an increasing mobility near midgap on the extrapolation of the subthreshold curve, (b) Voltage shifts determined from the second slope are identical to the dashed line from midgap to inversion.

\subsubsection{Doping}

Processing methods can be used to produce a nonuniform doping in the MOSFET channel regions. For MOSFETs produced on the same wafer with silicon sensors, vertical nonuniform doping occurs in case of p-spray implant isolation. This implant can increase the subthreshold swing depending on the depth of the implant $[28,166]$, but it will not cause irregularities in the swing. The initial increase in the swing should not affect the subthreshold charge separation technique of the comparison of pre- to post-irradiation curves. MOSFETs which have nonuniform doping in the lateral direction under the gate cause a bending of the conduction and valence bands with respect to the Fermi level. As a result, the entire silicon channel will not be at the same potential. It is necessary to determine an effective channel doping to use in Eq. 6.1. Assuming the device does not turn on until the entire channel is inverted, the effective channel doping would be the larger value near the source. However, in the present study the application of the method has not been tested for the case of nonuniform doping in the channel region.

\subsubsection{Interface and trapped charge assumptions}

Separation of radiation-induced threshold voltage shifts into $\Delta V_{o x}$ and $\Delta V_{i t}$ using the subthreshold charge separation technique is sensitive to the assumptions about the oxide trapped charge and interface traps. The subthreshold technique assumes that the interface traps are amphoteric [96]. Only acceptor traps are above midgap and donors below midgap. This provides the basis for assuming that all interface traps are neutral at the midgap potential, $V_{m g}[85,130]$. Assuming that only holes are trapped in the oxide (positive oxide charge), the midgap voltage shift, $\Delta V_{m g}$, is representative of the quantity of trapped holes in the oxide. Research has been performed which proposes the trapping of electrons in the oxide [107] or at the interface [167] in hardened oxides. The trapped electrons would simply have a canceling effect on the oxide trapped holes. In this case, the midgap voltage shift would represent the difference between the trapped 
holes and electrons and still provide a good tool for judging radiation hardness. Violation of the assumption that the interface traps are amphoteric would cause a non-neutral interface charge at midgap, but would not affect the linearity of the subthreshold characteristics. Research has been performed showing that interface traps are amphoteric $[96,168]$ and non-amphoteric [169]. In n-channel MOSFETs, donors above midgap and in p-channel MOSFETs, acceptors below midgap will result in an overestimation by the subthreshold technique of the oxide charge and an underestimation of the interface traps by the number of non-amphoteric traps [161].

\subsection{High-ohmic p- and n-MOSFETs}

Silicon sensors are produced on high-ohmic p- or n-doped material. The application of the subthreshold current technique on MOSFET test devices produced on the same wafer with the sensors, is a challenge. In this section is presented the midgap method of the subthreshold current technique and the determination of the oxide trapped charge, the interface traps and the density of interface traps as a function of energy in the Si bandgap on high-ohmic material. In order to perform the analysis, the following data set and parameters must be known, 1) the drain and/or source current (which best represents the channel current) as a function of gate voltage for gate voltages corresponding to below midgap through strong inversion. This data set may be taken either in the linear or saturated region provided $V_{d s}>>k T / q_{0}$. In the present analysis the data set have been taken in the linear region $\left(\left|V_{d s}\right|=50 \mathrm{mV}\right)$ in order to avoid the pinch off region in the MOSFET. In addition 2) the measurement of mobility, 3) the gate oxide thickness and 4) the interface channel region doping density. For an ideal MOSFET, the drain current will be composed almost entirely of the channel current under the gate. In this case, the drain current will follow an exponential dependence on gate voltage in the subthreshold with a single valued slope and will yield to ideal MOSFET subthreshold characteristics. In real MOSFETs, there are several other components of drain current that will result in a departure from the ideal subthreshold curve. These components include drain to substrate leakage, gate leakage, edge or sidewall leakage and back channel leakage. A technique for reducing the interference from leakage sources is to measure the source current, $I_{s}$, rather than the drain current, $I_{d}$. This will eliminate the drain to substrate leakage current, since source and substrate are usually common, and it may reduce other leakage components as well. Before a decision is made whether to monitor $I_{d}$ as a function of $V_{g a t e}$, or $I_{s}$ as a function of $V_{g a t e}$, the currents in all available leads of the MOSFET should be monitored as a function of $V_{\text {gate }}$ on the unirradiated samples. In the following data the drain-source current $I_{d s}$, has been measured from the source electrode, $I_{s}$, of the circular MOSFET design in order to avoid leakage current effects. To apply the subthreshold charge separation technique, a value for the channel mobility is required. From the parametrization mobility model presented in the previous chapter the strong inversion mobility as a function of gate voltage can be extracted. In the subthreshold region the mobility needed is the weak inversion value. Since, for a MOSFET, the mobility increases as the surface potential is lowered, the maximum strong inversion value should be used. The interface states degrade the mobility and it is expected that the post-irradiation mobility will be less that the pre-irradaition value. The subthreshold charge separation technique applied to low-ohmic MOSFETs has been introduced using the pre-irradiation mobility to analyze the post-irradiation data, and re-calculating the mobility for each post-irradiation data set [161]. If the pre-irradiation value is used, it is assumed that the weak inversion mobility changes little with irradiation or the mobility calculated from the post-irradiation curves are a worse estimate of the post-irradiation weak inversion mobility. Using the 
interface state neutrality, the mobility at midgap could be better approximated by the pre-irradiation value. At midgap, all interface states located above midgap have neutral charge, and it is assumed that there is no trapped oxide charge near the interface to affect the mobility. However according to Ref. [140] the mobility is affected by the oxide trapped charge at the $\mathrm{Si}-\mathrm{SiO}_{2}$ interface. So for the calculation of the midgap current in the pre- and post-irradiation data the extracted maximum mobility in strong inversion has been used in the analysis of high-ohmic MOSFETs. Fig. 6.6 (a) is an illustration of a typical pre- and post-irradiation plot of $I_{d s}$ as a function of $V_{\text {gate }}$ in log scale for a p-channel MOSFET showing the separation of $\Delta V_{\text {th }}$ into $\Delta V_{o x}$ and $\Delta V_{i t}$. In Fig. 6.6 (b) a n-channel pre- and post-irradiation subthreshold characteristics are shown.

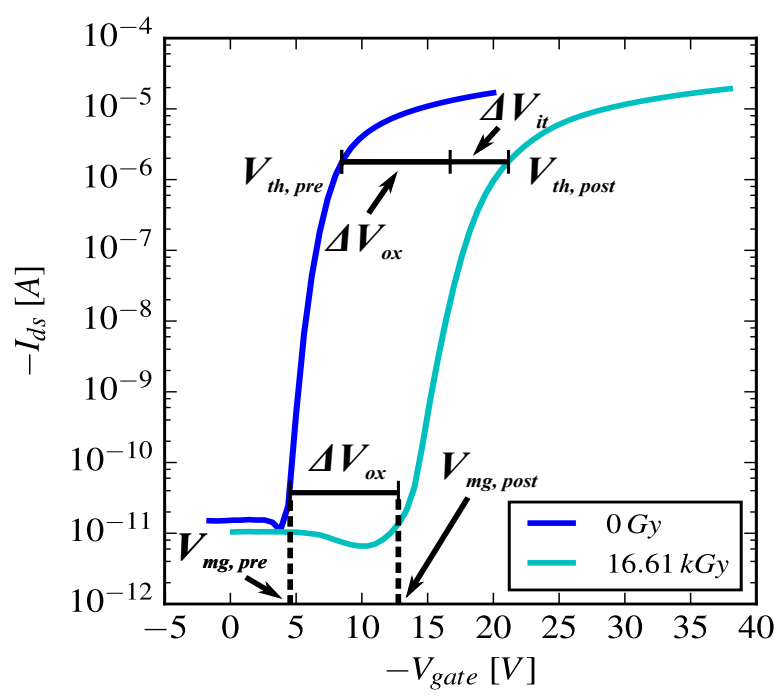

(a)

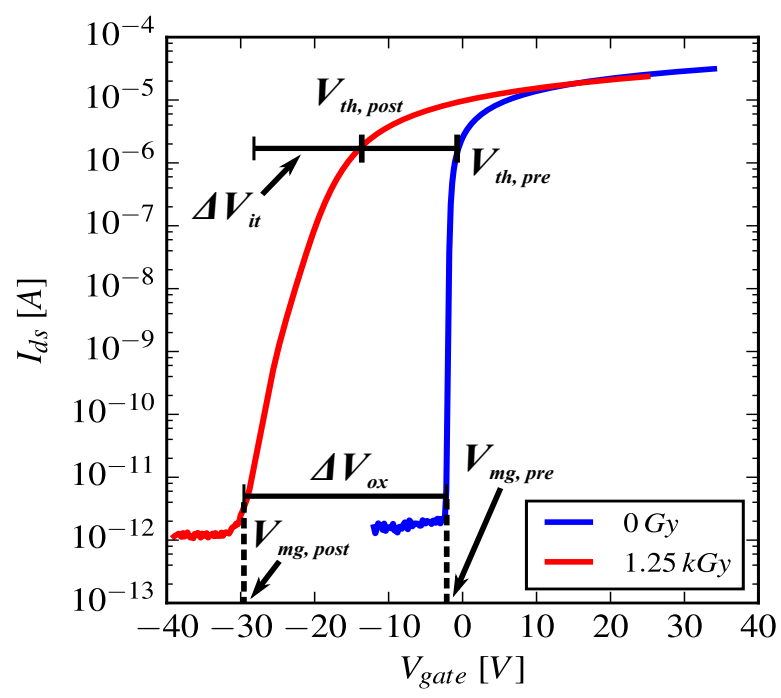

(b)

Figure 6.6: Subthreshold-current characteristics before and after X-ray irradiation (a) p-MOSFET $<111>$ and (b) n-MOSFET $<100>$.

\subsubsection{The p-MOSFET irradiated with $E_{\text {field }}$ pointing from the $\mathrm{Si}$ into the $\mathrm{SiO}_{2}$}

Using the Eqs. 6.1, 6.3 and assuming no interface traps the $I_{d s}^{\text {ideal }}\left(V_{\text {gate }}\right)$ subthreshold theoretical curve of p-MOSFET is calculated and presented in Fig. 6.7 (a). The $V_{t h}$ is extracted using the parametrization model fit, $I_{d s}^{f i t}$, in strong inversion as presented in Ch. 5. For the mobility value in weak inversion, $\mu$ in Eq. 6.1, the $\mu_{0}$ extracted from the parametrization model has been used. The channel midgap current, $I_{m g}=I_{d s}\left(\phi_{s}\right)$, is calculated at $\phi_{s}=\phi_{b}$. The horizontal $I_{m g}$ line crosses the pre-irradiation subthreshold $I_{d s}$ as a function of $V_{\text {gate }}$ characteristic. The gate voltage at $I_{m g}$ is $V_{m g}$. For high-ohmic material, with channel doping $N_{d} \approx 10^{12}$ $\mathrm{cm}^{-3}$, the calculated $I_{m g}$ value is in the pA range. This is an important difference between $I_{m g}$ values in high and low-ohmic material, Fig. 6.1. Allows the determination of interface trap density between pre-irradiation and the theoretical subthreshold curve. In addition, the energy distribution of the interface traps, $D_{i t}$, in the Si bandgap can be determined from the dependence of the gate voltage on the surface potential, $V_{\text {gate }}^{i t}\left(\phi_{s}\right)$. As presented in Fig. 6.7 (a) the measured subthreshold curve for a p-MOSFET has a different swing than the theoretical calculated curve, $I_{d s}^{\text {ideal }}$, due to the presence of donor traps above the midgap, Fig. 6.7 (b). Similar to the Terman technique [170] the density of interface traps $D_{i t}\left[\mathrm{states} /\left(\mathrm{V} \cdot \mathrm{cm}^{2}\right)\right]$, as a function of the 
surface potential $\phi_{s}[\mathrm{~V}]$ is calculated from Eq. 6.16 where $V_{\text {gate }}^{i t}$ is the applied gate bias for the measured data and $V_{\text {gate }}^{\text {ideal }}$ the gate bias of the ideal subthreshold curve. According to Eq. $6.3 d V_{\text {gate }}^{\text {ideal }} / d \phi_{s} \approx 1$, so the $D_{i t}$ is calculated from Eq. 6.17.

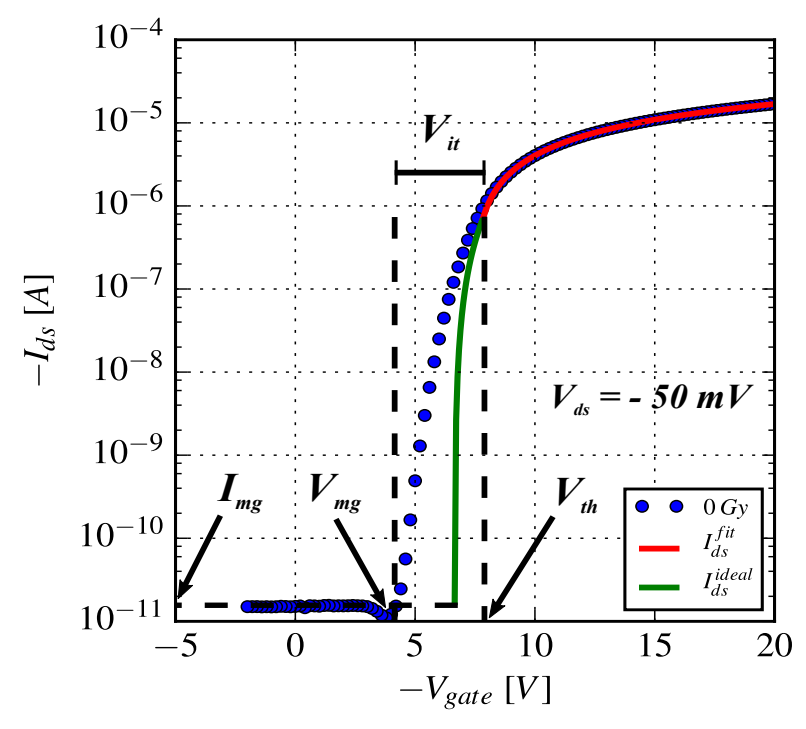

(a)

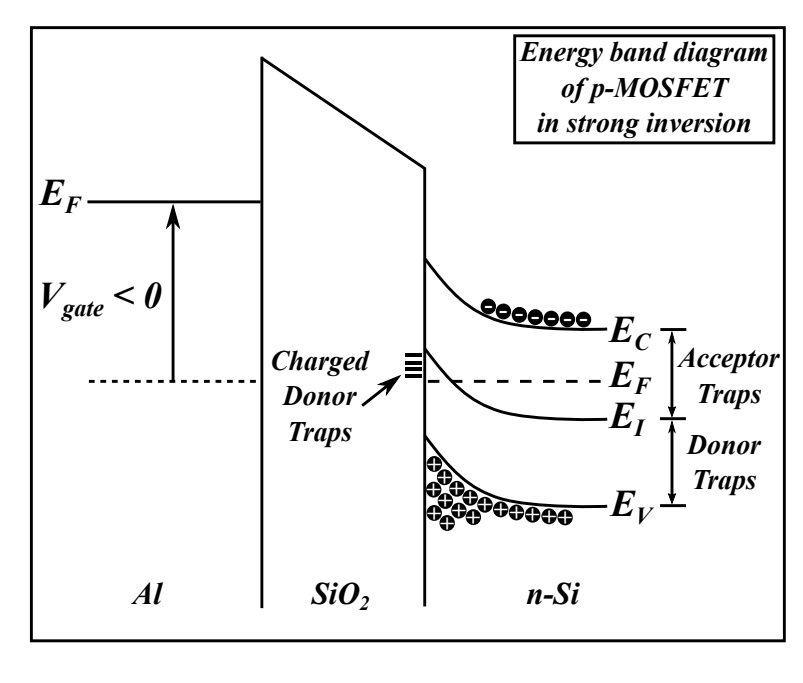

(b)

Figure 6.7: (a) $I_{d s}\left(V_{\text {gate }}\right)$ data before X-ray irradiation, $I_{d s}^{\text {ideal }}\left(V_{\text {gate }}\right)$ subthreshold theoretical curve, and $I_{d s}^{f i t}\left(V_{\text {gate }}\right)$ parametrization model in strong inversion for a p-MOSFET $<111>$ and (b) Energy band diagram of a p-MOSFET in strong inversion showing donor traps.

$$
\begin{gathered}
D_{i t}\left(\phi_{s}\right)=\frac{C_{o x}}{q_{0}}\left(\frac{d V_{\text {gate }}^{i t}}{d \phi_{s}}-\frac{d V_{\text {gate }}^{\text {ideal }}}{d \phi_{s}}\right) \\
D_{i t}\left(\phi_{s}\right)=\frac{C_{o x}}{q_{0}}\left(\frac{d V_{\text {gate }}^{i t}}{d \phi_{s}}-1\right)
\end{gathered}
$$

In Fig. 6.8 (a) is presented the $I_{d s}\left(V_{\text {gate }}\right)$ as a function of X-ray dose in the subthreshold region. The increase in "stretchout" as a function of X-ray dose, $V_{i t}$, of the subthreshold curves along the gate voltage axis is caused by the increase of occupied interface traps in the Si bandgap between midgap and threshold. For a p-MOSFET (n-bulk Si) the subthreshold characteristics are influenced by the increase of donor traps below midgap (lower part of Si bandgap). The midgap method has been used to determine the contribution of fixed oxide charge and interface traps to the effective oxide charge which has been calculated using the extracted threshold voltage, Fig. 6.8 (b). The midgap voltage $V_{m g}$, is determined after the calculation of the midgap current, $I_{m g}$, for each subthreshold curve. The midgap mobility is required for the calculation of the $I_{m g}$. Since, for a MOSFET, the mobility increases as the surface potential is lowered, the maximum strong inversion value should be used [161]. The mobility $\mu_{0}$ values extracted in strong inversion, Fig. 6.8 (b), are used for the calculation of $I_{m g}$. As presented in Ch. 5, $\mu_{0}$ decreases, so $\left|I_{m g}\right|$ decreases with increasing dose. The calculated $I_{m g}$ and extracted $V_{m g}$ values are presented in Table 6.1. The fixed oxide charge density, $N_{o x}$, is calculated from the Eq. 6.18. The voltage difference between threshold and midgap is related to the 
contribution of donor interface traps to the effective oxide charge. This voltage difference $V_{i t}$, is calculated from the Eq. 6.19. Similar to the oxide charge density, the interface trap density, $N_{i t}$, is calculated from Eq. 6.20 with (-) for n-bulk Si.

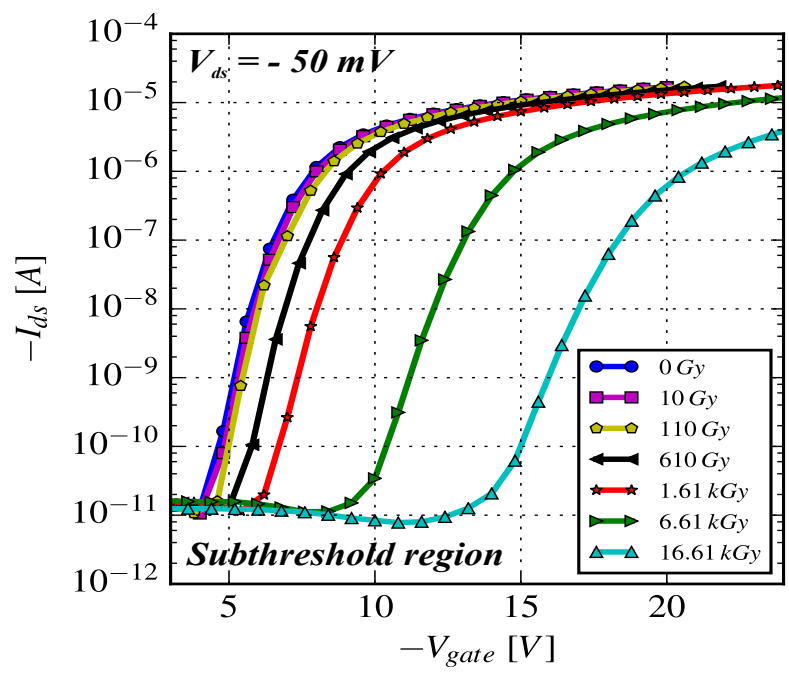

(a)

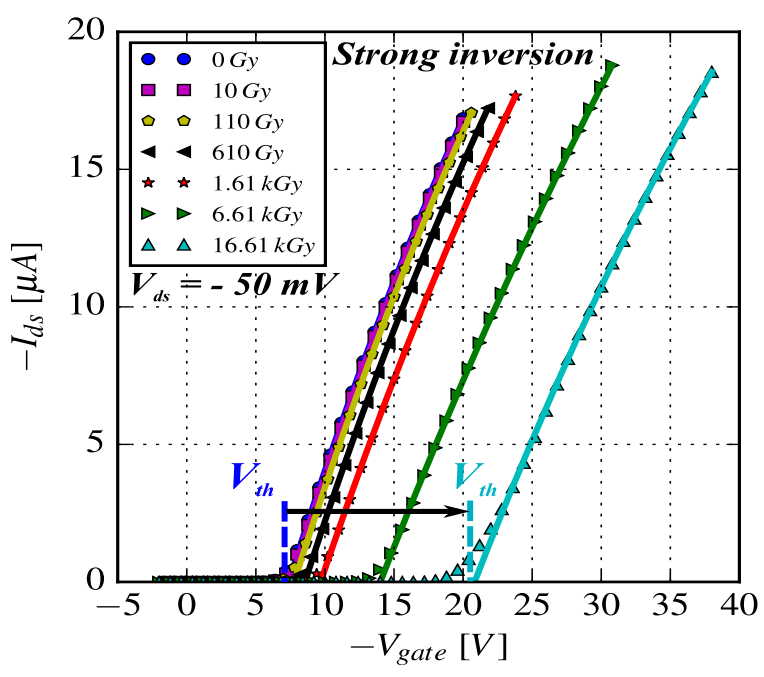

(b)

Figure 6.8: (a) $I_{d s}\left(V_{\text {gate }}\right)$ in the subthreshold region, (b) $I_{d s}\left(V_{\text {gate }}\right)$ in strong inversion and the parametrization model fit used to determine the threshold voltage, $V_{t h}$, before and after X-ray irradiation of the p-MOSFET $<111>$ irradiated with $E_{\text {field }} \uparrow$.

$$
\begin{gathered}
N_{o x}=\frac{C_{o x}\left(-V_{m g}+\phi_{b}+\phi_{m s}\right)}{q_{0}} \\
V_{i t}=V_{t h}-V_{m g} \\
N_{i t}=\frac{C_{o x}\left(-V_{i t}+\phi_{b} \mp \frac{\sqrt{2 \varepsilon_{s i} \varepsilon_{0} q_{0} N_{d} 2\left|\phi_{b}\right|}}{C_{o x}}\right)}{q_{0}}
\end{gathered}
$$

Table 6.1: Charge separation results as a function of X-ray dose of the p-MOSFET $<111>$ irradiated with $E_{\text {field }} \uparrow$.

\begin{tabular}{llllllll}
\hline Dose $[\mathrm{Gy}]$ & 0 & 10 & 110 & 610 & 1610 & 6610 & 16610 \\
\hline$V_{\text {inv }}^{\text {inr }}[\mathrm{V}]$ & -20.0 & -20.0 & -20.0 & -20.6 & -21.8 & -23.8 & -29.0 \\
$E_{\text {fir }}^{\text {iield }}[\mathrm{kV} / \mathrm{cm}]$ & -500 & -500 & -490 & -500 & -500 & -460 & -440 \\
$-I_{m g}[\mathrm{pA}]$ & 12.95 & 13.04 & 12.93 & 12.57 & 11.94 & 10.42 & 10.04 \\
$-V_{\text {th }}[\mathrm{V}]$ & 7.39 & 7.54 & 7.79 & 8.54 & 9.62 & 13.71 & 19.85 \\
$-V_{\text {mg }}[\mathrm{V}]$ & 4.0 & 4.2 & 4.4 & 5.0 & 5.6 & 8.2 & 12.0 \\
$-V_{\text {it }}[\mathrm{V}]$ & 3.39 & 3.34 & 3.39 & 3.54 & 4.02 & 5.51 & 7.85 \\
$N_{\text {off }}^{\text {eff }}\left[10^{10} \mathrm{~cm}^{-2}\right]$ & 57.9 & 59.3 & 61.4 & 67.8 & 77.2 & 112.4 & 165.3 \\
$N_{\text {ox }}\left[10^{10} \mathrm{~cm}^{-2}\right]$ & 29.8 & 31.6 & 33.3 & 38.5 & 43.6 & 66.1 & 98.8 \\
$N_{i t}\left[10^{10} \mathrm{~cm}^{-2}\right]$ & 28.1 & 27.7 & 28.1 & 29.4 & 33.5 & 46.3 & 66.5 \\
$\bar{D}_{\text {it }}\left[10^{12} \mathrm{eV}^{-1} \mathrm{~cm}^{-2}\right]$ & 1.16 & 1.06 & 1.07 & 1.16 & 1.56 & 1.80 & 2.79 \\
\hline
\end{tabular}




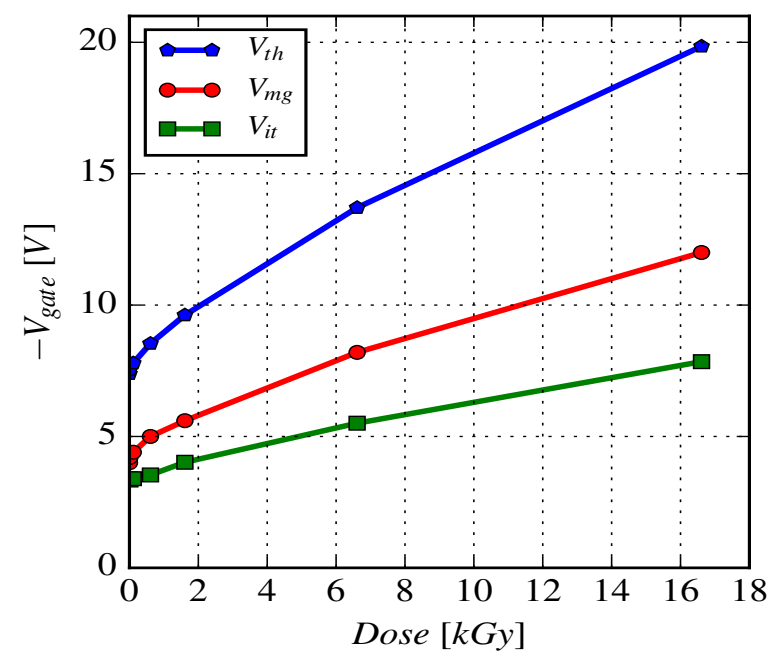

(a)

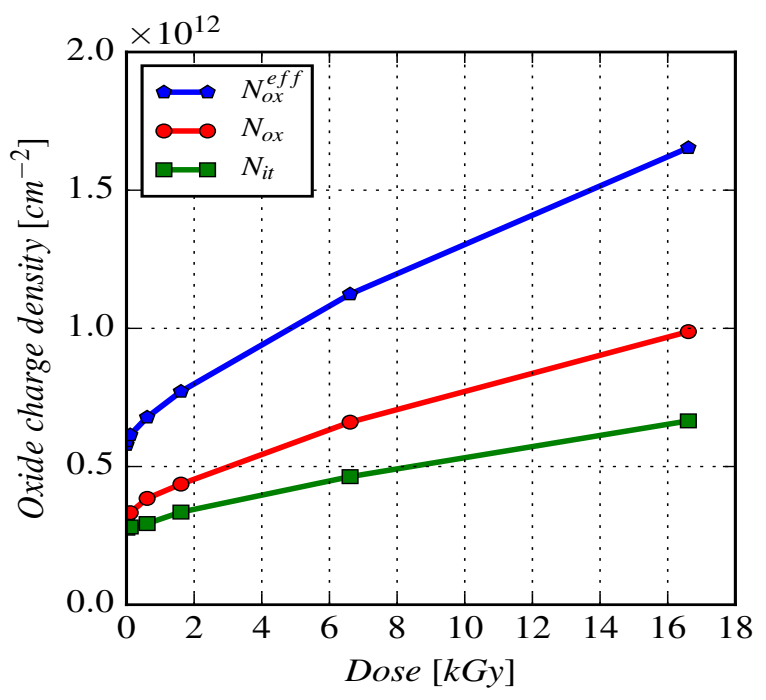

(b)

Figure 6.9: (a) Gate voltage and, (b) Oxide charge density as a function of X-ray dose of the p-MOSFET $<111>$ irradiated with $E_{\text {field }} \uparrow$.

The dose dependence of $V_{t h}, V_{m g}$ and $V_{i t}$ is presented in Fig. 6.9 (a) and the calculated $N_{o x}^{e f f}, N_{o x}$ and $N_{i t}$ in Fig. 6.9 (b). $V_{t h}$ shifts from $\approx-7.4 \mathrm{~V}$ to $\approx-19.9 \mathrm{~V}$ up to an X-ray dose of $16.61 \mathrm{kGy}$. The contribution of $V_{m g}$ and $V_{i t}$ to the net $V_{t h}$ shift is from $-4.0 \mathrm{~V}$ to $-12.0 \mathrm{~V}$ and $\approx-3.4 \mathrm{~V}$ to $\approx-7.9 \mathrm{~V}$ respectively. The change in $V_{t h}$ is explained by the additive effect of positive charge contribution of interface traps and positive fixed oxide charge to the total net $V_{t h}$ negative shift. In particular, $N_{o x}^{e f f}$ increases from $\approx 5.8 \cdot 10^{11} \mathrm{~cm}^{-2}$ to $\approx 1.7 \cdot 10^{12} \mathrm{~cm}^{-2}, N_{o x}$ increases from $\approx 3.0 \cdot 10^{11} \mathrm{~cm}^{-2}$ to $\approx 9.9 \cdot 10^{11} \mathrm{~cm}^{-2}$ and $N_{i t}$ increases from $\approx 2.8 \cdot 10^{11} \mathrm{~cm}^{-2}$ to $\approx 6.7 \cdot 10^{11} \mathrm{~cm}^{-2}$. In order to determine the gate voltage dependence on the surface potential, $V_{\text {gate }}^{\text {it }}\left(\phi_{s}\right)$, between midgap and threshold the non-linear equation $I_{d s}^{\text {ideal }}\left(\phi_{s}\right)-I_{d s}=0$ has been solved.

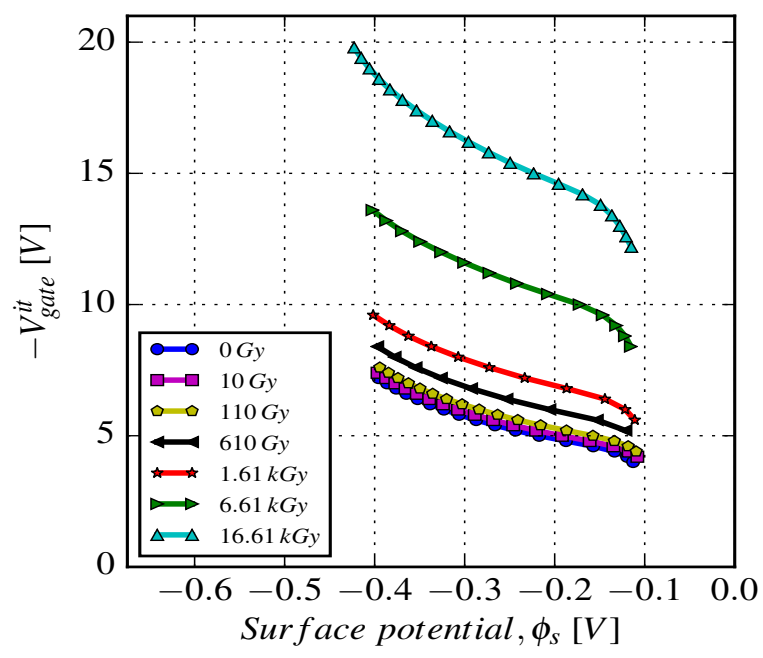

Figure 6.10: Gate voltage as a function of surface potential in the subthreshold region before and after X-ray irradiation of the p-MOSFET $<111>$ irradiated with $E_{\text {field }} \uparrow$. 
The solution gives the surface potential values, $\phi_{s}$, and the $V_{\text {gate }}^{i t}\left(\phi_{s}\right)$ as a function of X-ray dose, which is presented in Fig. 6.10. The slope of the $V_{\text {gate }}^{i t}\left(\phi_{s}\right)$ curves is related to the response of the surface potential to the gate voltage. The presence of interface traps causes the surface potential to be less sensitive to changes in gate voltage and will result in a increase in slope of $V_{\text {gate }}^{i t}\left(\phi_{S}\right)[171,172]$. The density of interface traps as a function of surface potential, $D_{i t}\left(\phi_{s}\right)$, is calculated from Eq. 6.17 and presented in Fig. 6.11 (a). So far with this method has been extracted the energy distribution of interface traps (donor traps) between midgap, $\phi_{s}=\phi_{b}$ and threshold or strong inversion $\left|\phi_{s}\right| \geq\left|2 \phi_{b}\right|$ for the p-MOSFET. In Fig. 6.11 (b) is presented the energy distribution of interface traps, $D_{i t}\left(E_{i t}\right)$, where $E_{i t}=q_{0}\left(\phi_{s}-\phi_{b}\right)$ is calculated from the middle of the Si bandgap. The integrated $D_{i t}$ values, between midgap and threshold, correspond to the $N_{i t}$ values extracted for each X-ray dose, presented in Fig. 6.9 (b).

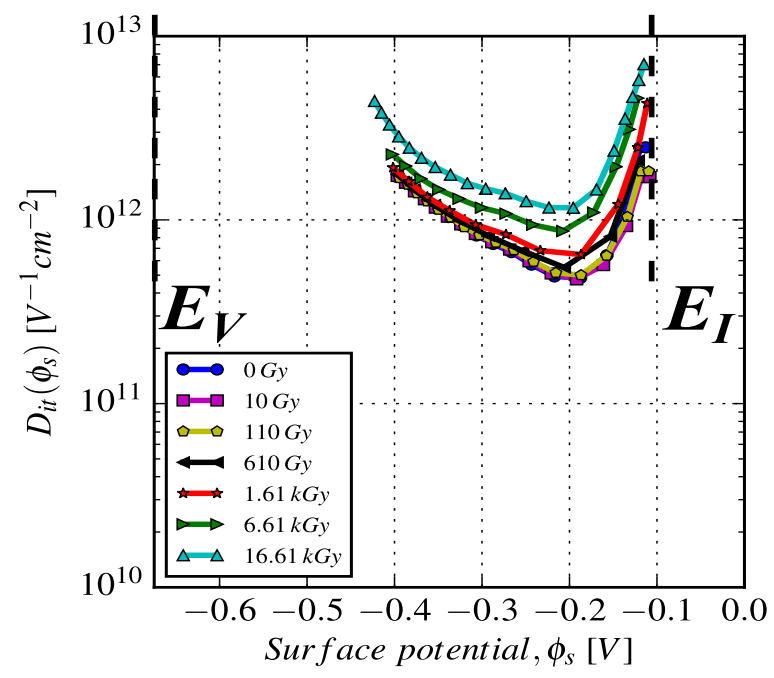

(a)

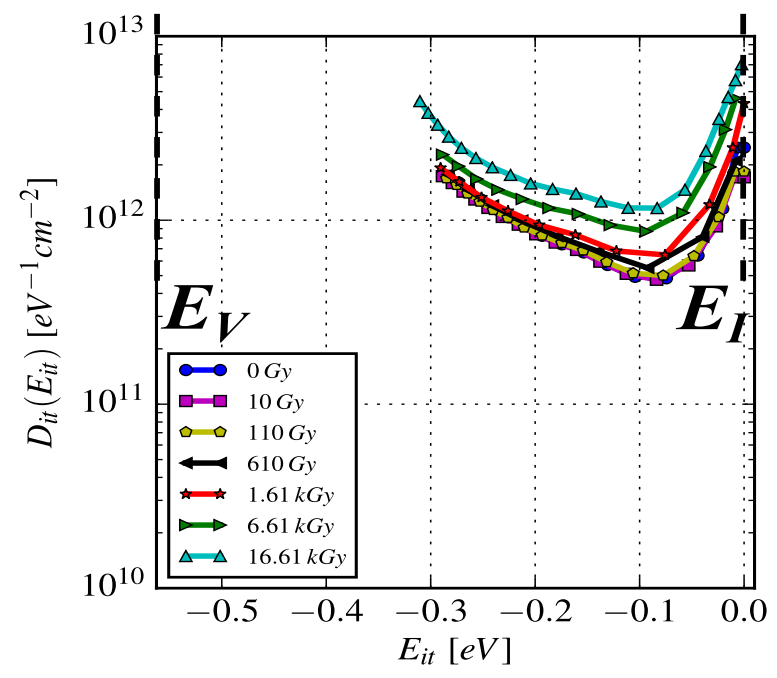

(b)

Figure 6.11: (a) $D_{i t}$ as a function of the surface potential and, (b) Energy in the Si bandgap before and after $\mathrm{X}$-ray irradiation of the p-MOSFET $<111>$ irradiated with $E_{\text {field }} \uparrow$.

The $D_{i t}$ values in the midgap position increase from $\approx 2.0 \cdot 10^{12} \mathrm{eV}^{-1} \mathrm{~cm}^{-2}$ to $\approx 7.0 \cdot 10^{12} \mathrm{eV}^{-1} \mathrm{~cm}^{-2}$ and at threshold $0.3 \mathrm{eV}$ below midgap the increase is from $\approx 2.0 \cdot 10^{12} \mathrm{eV}^{-1} \mathrm{~cm}^{-2}$ to $\approx 5.0 \cdot 10^{12} \mathrm{eV}^{-1} \mathrm{~cm}^{-2}$ up to $\mathrm{X}$-ray dose of $16.61 \mathrm{kGy}$. The average value of $\bar{D}_{i t}$ in the energy range between midgap and threshold increases from $\approx 1.2 \cdot 10^{12} \mathrm{eV}^{-1} \mathrm{~cm}^{-2}$ to $\approx 2.8 \cdot 10^{12} \mathrm{eV}^{-1} \mathrm{~cm}^{-2}$ and is higher than the integrated interface trap density, $N_{i t}$. The energy distribution of interface traps shows a minimum $0.1 \mathrm{eV}$ below midgap, $E_{I}$, and then increases towards the valence band edge, $E_{V}$. The results suggest a half "U" shaped distribution of traps, in the lower part of the Si bandgap with a "peak" at midgap. Unfortunately with the subthreshold current technique applied on p-MOSFET, the $D_{i t}$ in the upper part of the bandgap cannot be extracted.

\subsubsection{The p-MOSFET irradiated with $E_{\text {field }}$ pointing from the $\mathrm{SiO}_{2}$ into the $\mathrm{Si}$}

Similar to the analysis presented in the previous section, the fixed oxide charge density, the interface trap density and the energy distribution of interface traps are extracted from the p-MOSFET $<111>$ irradiated with $E_{\text {field }}$ pointing $\downarrow$ from the $\mathrm{SiO}_{2}$ into the Si. In Fig. 6.12 (a) is presented the $I_{d s}\left(V_{\text {gate }}\right)$ in log scale as 
a function of X-ray dose in the subthreshold region. As mentioned above for the p-MOSFET, the increase in "stretchout" as a function of X-ray dose of the subthreshold curves is caused by the increase of interface traps in the lower part of the Si bandgap.

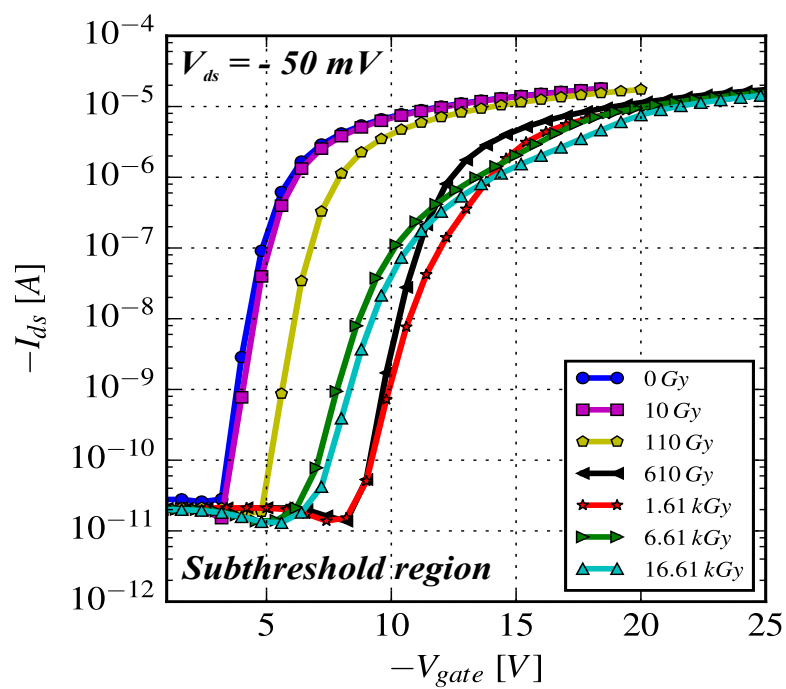

(a)

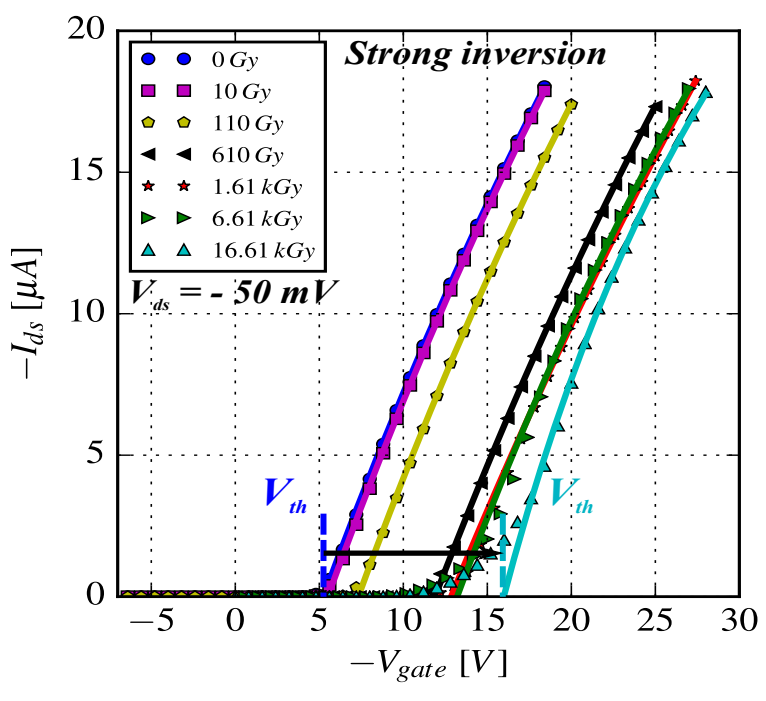

(b)

Figure 6.12: (a) $I_{d s}\left(V_{\text {gate }}\right)$ in the subthreshold region, (b) $I_{d s}\left(V_{\text {gate }}\right)$ in strong inversion and the parametrization model fit used to determine the threshold voltage, $V_{t h}$, before and after X-ray irradiation of the pMOSFET $<111>$ irradiated with $E_{\text {field }} \downarrow$.

Using the midgap method, the midgap voltage, $V_{m g}$, is determined after the calculation of the midgap current, $I_{m g}$, for each subthreshold curve. However for this case the mobility, $\mu_{0}$, values extracted in the onset of strong inversion $\left(E_{\text {field }} \approx 0\right.$ ), Fig. 6.12 (b), remain constant with increasing dose and increase only for the highest dose steps, Ch. 5. As shown in Fig. 6.3 (b), $\mu_{0}$ probably increases due to decrease of minority carrier fluctuations after the increase of interface traps. In the subthreshold curves, Fig. 6.12 (a), the effect is observed as a second swing around $V_{\text {gate }} \approx-15 \mathrm{~V}$ for the X-ray doses of 6.61 and $16.61 \mathrm{kGy}$. In order to overcome this non-usual $\mu_{0}$ behavior as a function of X-ray dose, for the calculation of $I_{m g}$ the Si bulk hole mobility is used $\approx 450 \mathrm{~cm}^{2} /(\mathrm{Vs})$. As discussed above the weak inversion mobility cannot be larger than the bulk mobility.

Table 6.2: Charge separation results as a function of X-ray dose of the p-MOSFET $<111>$ irradiated with

\begin{tabular}{llllllll} 
& \multicolumn{7}{c}{$E_{\text {field } \downarrow .}$} \\
\hline Dose $[\mathrm{Gy}]$ & 0 & 10 & 110 & 610 & 1610 & 6610 & 16610 \\
\hline$V_{\text {acc }}^{\text {irr }}[\mathrm{V}]$ & 7.0 & 7.0 & 7.0 & 6.0 & 1.0 & 0 & 0 \\
$E_{\text {field }}^{\text {ir }}[\mathrm{kV} / \mathrm{cm}]$ & 490 & 500 & 540 & 520 & 460 & 510 & 570 \\
$-I_{m g}[\mathrm{pA}]$ & 22.63 & 22.63 & 22.63 & 22.63 & 22.63 & 22.63 & 22.63 \\
$-V_{\text {th }}[\mathrm{V}]$ & 5.25 & 5.50 & 7.32 & 11.89 & 12.84 & 13.20 & 15.97 \\
$-V_{m g}[\mathrm{~V}]$ & 3.0 & 3.4 & 4.8 & 8.6 & 8.6 & 6.2 & 6.6 \\
$-V_{\text {it }}[\mathrm{V}]$ & 2.25 & 2.1 & 2.52 & 3.29 & 4.24 & 7.0 & 9.37 \\
$N_{\text {off }}^{\text {eff }}\left[10^{10} \mathrm{~cm}^{-2}\right]$ & 39.5 & 41.7 & 57.3 & 96.7 & 105.0 & 108.0 & 131.9 \\
$N_{\text {ox }}\left[10^{10} \mathrm{~cm}^{-2}\right]$ & 21.2 & 24.7 & 36.7 & 69.5 & 69.5 & 48.8 & 52.3 \\
$N_{i t}\left[10^{10} \mathrm{~cm}^{-2}\right]$ & 18.3 & 16.7 & 20.6 & 27.2 & 35.5 & 59.2 & 79.7 \\
$\bar{D}_{\text {it }}\left[10^{12} \mathrm{eV}^{-1} \mathrm{~cm}^{-2}\right]$ & 0.91 & 0.65 & 0.80 & 1.06 & 1.58 & 2.94 & 4.30 \\
\hline
\end{tabular}




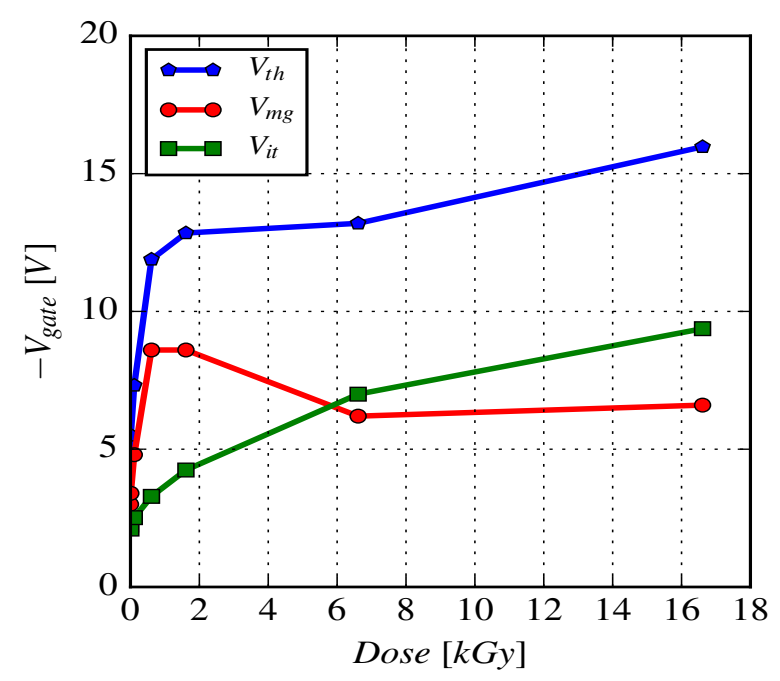

(a)

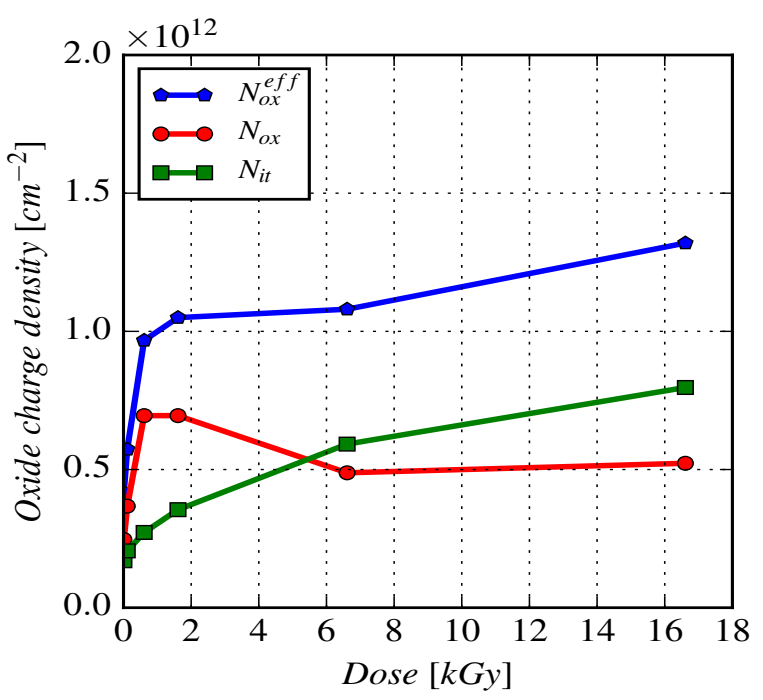

(b)

Figure 6.13: (a) Gate voltage and, (b) Oxide charge density as a function of X-ray dose of the p-MOSFET $<111>$ irradiated with $E_{\text {field }} \downarrow$.

The calculated $I_{m g}$ and extracted $V_{m g}$ values are presented in Table 6.2. The fixed oxide charge density, $N_{o x}$, is calculated from the Eq. 6.18. The $V_{i t}$ "stretchout" is related to the presence of donor traps between midgap and threshold and calculated from Eq. 6.19, where the interface trap density, $N_{i t}$, is calculated from Eq. 6.20 with (-) for n-bulk Si. The dose dependence of $V_{t h}, V_{m g}$ and $V_{i t}$ is presented in Fig. 6.13 (a) and the calculated $N_{o x}^{e f f}, N_{o x}$ and $N_{i t}$ in Fig. 6.13 (b) for the p-MOSFET $<111>$ irradiated with $E_{\text {field }} \downarrow$. $V_{t h}$ shifts from $\approx-5.3 \mathrm{~V}$ to $\approx-16.0 \mathrm{~V}$ up to $\mathrm{X}$-ray dose of $16.61 \mathrm{kGy}$. The contribution of $V_{m g}$ and $V_{i t}$ to the net shift is from $-3.0 \mathrm{~V}$ to $-6.6 \mathrm{~V}$ and from $\approx-2.3 \mathrm{~V}$ to $\approx-9.4 \mathrm{~V}$ respectively.

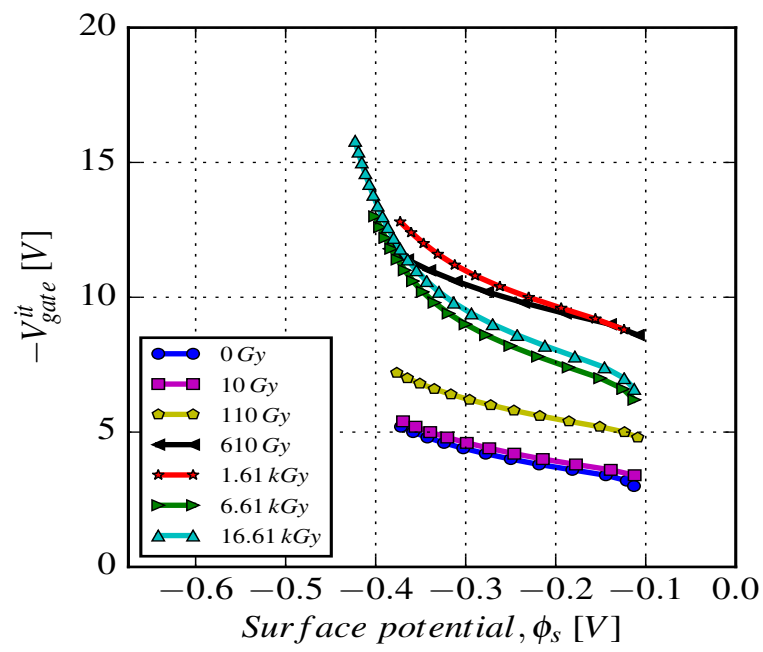

Figure 6.14: Gate voltage as a function of surface potential in the subthreshold region before and after X-ray irradiation of the p-MOSFET $<111>$ irradiated with $E_{\text {field }} \downarrow$.

Similar as for p-MOSFET irradiated with $E_{\text {field }} \uparrow$, the change in $V_{t h}$ is explained by the additive effect of positive charge contribution of interface traps and positive fixed oxide charge to the total net $V_{t h}$. So 
far, $N_{o x}^{e f f}$ increases from $\approx 4.0 \cdot 10^{11} \mathrm{~cm}^{-2}$ to $\approx 1.3 \cdot 10^{12} \mathrm{~cm}^{-2}, N_{o x}$ increases from $\approx 2.1 \cdot 10^{11} \mathrm{~cm}^{-2}$ to $\approx 5.2 \cdot 10^{11} \mathrm{~cm}^{-2}$ and $N_{i t}$ increases from $\approx 1.8 \cdot 10^{11} \mathrm{~cm}^{-2}$ to $\approx 8.0 \cdot 10^{11} \mathrm{~cm}^{-2}$. The $V_{\text {gate }}^{i t}\left(\phi_{s}\right)$ between midgap and threshold is determined after solving the non-linear equation $I_{d s}^{\text {ideal }}\left(\phi_{s}\right)-I_{d s}=0$. The solution gives the surface potential values, $\phi_{s}$, and the $V_{\text {gate }}^{i t}\left(\phi_{s}\right)$ as a function of X-ray dose is presented in Fig. 6.14. The slope of the $V_{\text {gate }}^{i t}\left(\phi_{S}\right)$ curves increases as a function of dose due to the increase of interface traps. The density of interface traps as a function of surface potential, $D_{i t}\left(\phi_{s}\right)$, is calculated from Eq. 6.17 and presented in Fig. 6.15 (a). In addition, the energy distribution of interface traps, $D_{i t}\left(E_{i t}\right)$, where $E_{i t}=q_{0}\left(\phi_{s}-\phi_{b}\right)$ is calculated from the middle of the Si bandgap is presented in Fig. 6.15 (b).

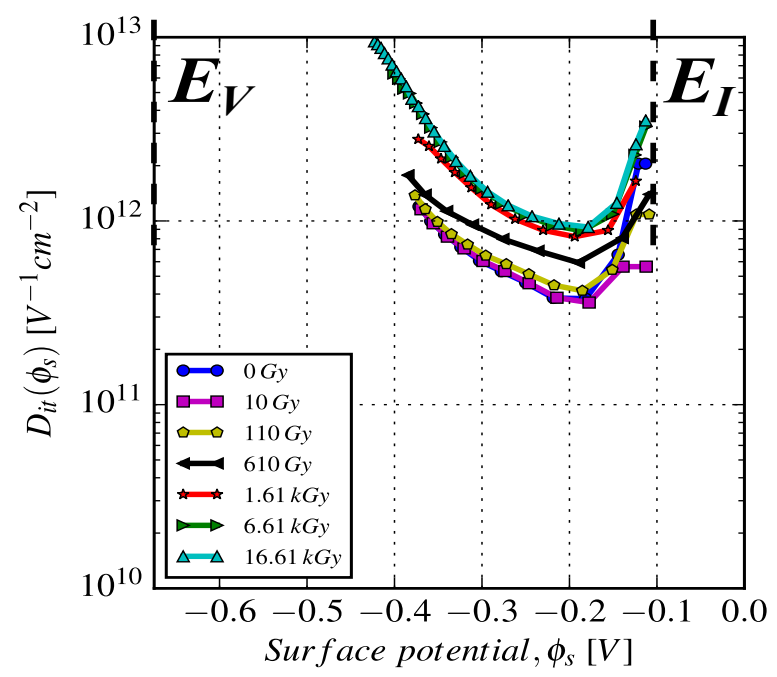

(a)

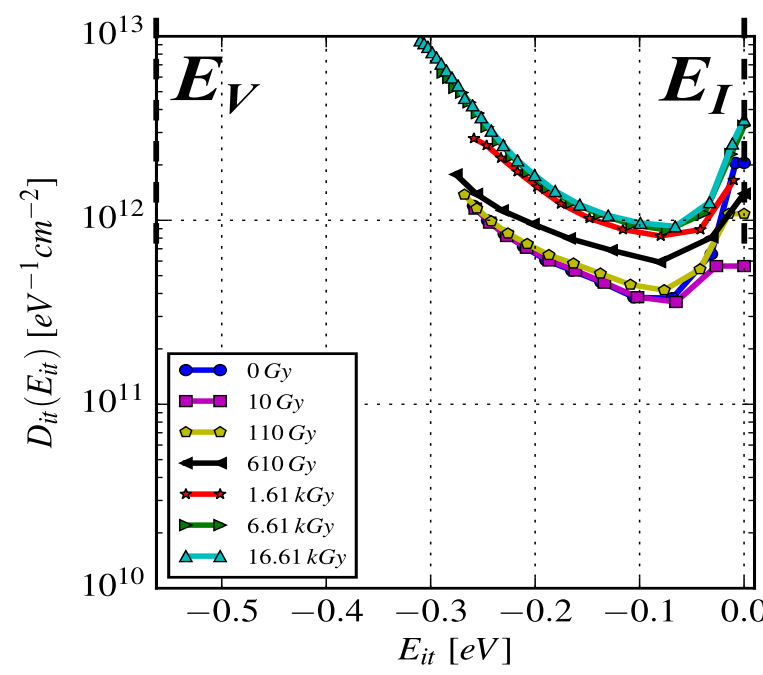

(b)

Figure 6.15: (a) $D_{i t}$ as a function of the surface potential and, (b) Energy in the Si bandgap before and after X-ray irradiation of the p-MOSFET $<111>$ irradiated with $E_{\text {field }} \downarrow$.

The $D_{i t}$ values in the midgap position decrease from $\approx 2.0 \cdot 10^{12} \mathrm{eV}^{-1} \mathrm{~cm}^{-2}$ to $\approx 6.0 \cdot 10^{11} \mathrm{eV}^{-1} \mathrm{~cm}^{-2}$ and for the last irradiation steps increase up to $\approx 3.5 \cdot 10^{12} \mathrm{eV}^{-1} \mathrm{~cm}^{-2}$. At the energy position $0.3 \mathrm{eV}$ below midgap (threshold) the increase is from $\approx 1.2 \cdot 10^{12} \mathrm{eV}^{-1} \mathrm{~cm}^{-2}$ to $\approx 1.0 \cdot 10^{13} \mathrm{eV}^{-1} \mathrm{~cm}^{-2}$ up to X-ray dose of $16.61 \mathrm{kGy}$. The average value of $\bar{D}_{i t}$ in the energy range between midgap and threshold increases from $\approx 0.9 \cdot 10^{12} \mathrm{eV}^{-1} \mathrm{~cm}^{-2}$ to $\approx 4.3 \cdot 10^{12} \mathrm{eV}^{-1} \mathrm{~cm}^{-2}$ and is higher than the integrated interface trap density, $N_{i t}$. The energy distribution of interface traps shows a minimum $0.1 \mathrm{eV}$ below midgap, $E_{I}$, and then increases towards the valence band edge, $E_{V}$. The results suggest a half "U" shaped distribution of traps, in the lower part of the Si bandgap with a "peak" at midgap.

\subsubsection{The n-MOSFET irradiated with $E_{\text {field }}$ pointing from the $\mathrm{Si}$ into the $\mathrm{SiO}_{2}$}

In addition, the midgap method is used on the n-MOSFET $<100>$ irradiated with $E_{\text {field }}$ pointing $\uparrow$ from the Si to the $\mathrm{SiO}_{2}$. In Fig. 6.16 (a) is presented the $I_{d s}\left(V_{\text {gate }}\right)$ in log scale as a function of X-ray dose in the subthreshold region. The increase in "stretchout" as a function of X-ray dose, $V_{i t}$, of the subthreshold curves along the gate voltage axis is caused by the increase of occupied interface traps in the Si bandgap between midgap and threshold. For the n-MOSFET (p-bulk Si) the subthreshold characteristics are influenced by 
the increase of acceptor traps above midgap (upper part of Si bandgap). In particular as the gate voltage is increased towards threshold, the distribution of electrons which are held by the interface traps will increase. Thus at threshold, the net change in the charge contribution due to interface traps compared to the net charge by fixed oxide charge will be negative. This negative charge will contribute to a positive shift in n-MOSFET threshold voltage [171, 172].

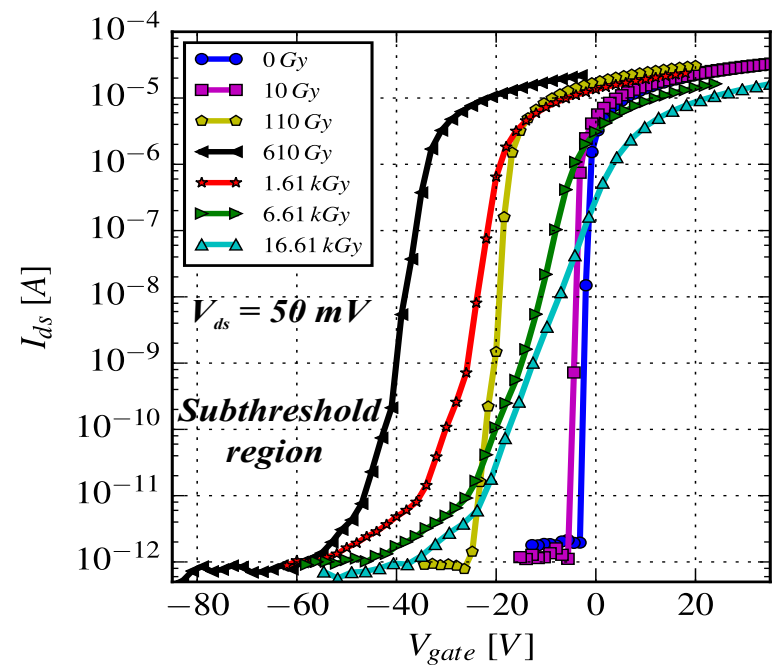

(a)

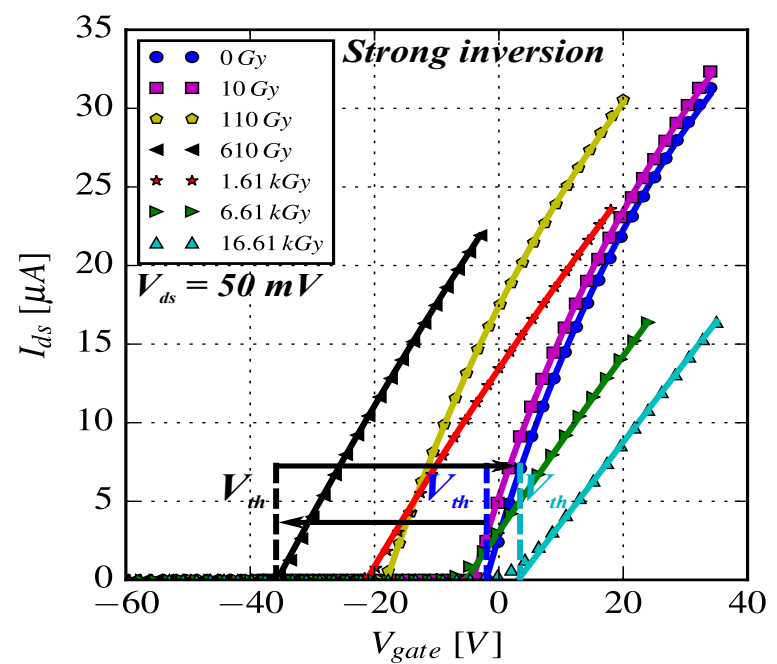

(b)

Figure 6.16: (a) $I_{d s}\left(V_{\text {gate }}\right)$ in the subthreshold region, (b) $I_{d s}\left(V_{\text {gate }}\right)$ in strong inversion and the parametrization model fit used to determine the threshold voltage, $V_{t h}$, before and after X-ray irradiation of the n-

MOSFET $<100>$ irradiated with $E_{\text {field }} \uparrow$.

With the midgap method is determined the contribution of fixed oxide charge and interface traps to the effective oxide charge which has been calculated from the extracted threshold voltage, Fig. 6.16 (b). The midgap voltage $V_{m g}$, is determined after the calculation of the midgap current, $I_{m g}$, for each subthreshold curve. The mobility $\mu_{0}$ values extracted in strong inversion, Fig. 6.16 (b), are used for the calculation of $I_{m g}$. As presented in Ch. 5, $\mu_{0}$ decreases, so $I_{m g}$ decreases with increasing dose.

Table 6.3: Charge separation results as a function of X-ray dose of the n-MOSFET $<100>$ irradiated with

\begin{tabular}{llllllll}
\multicolumn{7}{c}{$E_{\text {field }} \uparrow}$. \\
\hline Dose $[\mathrm{Gy}]$ & 0 & 10 & 110 & 610 & 1610 & 6610 & 16610 \\
\hline$V_{\text {acc }}^{\text {irr }}[\mathrm{V}]$ & -38.0 & -38.0 & -40.0 & -55.0 & -76.0 & -62.0 & -50.0 \\
$E_{\text {fir }}^{\text {field }}[\mathrm{kV} / \mathrm{cm}]$ & -510 & -500 & -520 & -600 & -590 & -680 & -740 \\
$I_{m g}[\mathrm{pA}]$ & 9.5 & 8.96 & 8.06 & 5.04 & 4.55 & 3.95 & 3.52 \\
$V_{\text {th }}[\mathrm{V}]$ & -2.06 & -4.23 & -18.04 & -35.66 & -21.29 & -5.29 & 3.07 \\
$V_{\text {mg }}[\mathrm{V}]$ & -2.4 & -5.0 & -23.6 & -48.6 & -40.4 & -32.0 & -27.4 \\
$V_{i t}[\mathrm{~V}]$ & 0.34 & 0.77 & 5.56 & 12.94 & 19.11 & 26.71 & 30.47 \\
$N_{\text {ox }}^{\text {eff }}\left[10^{10} \mathrm{~cm}^{-2}\right]$ & 5.6 & 12.3 & 54.8 & 109.0 & 64.8 & 15.6 & -10.2 \\
$N_{\text {ox }}\left[10^{10} \mathrm{~cm}^{-2}\right]$ & 5.7 & 13.7 & 71.0 & 150.0 & 122.7 & 96.9 & 82.7 \\
$N_{\text {it }}\left[10^{10} \mathrm{~cm}^{-2}\right]$ & -0.1 & -1.4 & -16.2 & -38.9 & -57.9 & -81.3 & -92.9 \\
$\bar{D}_{i t}\left[10^{12} \mathrm{eV}^{-1} \mathrm{~cm}^{-2}\right]$ & 0.02 & 0.10 & 0.67 & 1.58 & 2.51 & 2.39 & 2.58 \\
\hline
\end{tabular}

The calculated $I_{m g}$ and extracted $V_{m g}$ values are presented in Table 6.3. The fixed oxide charge density, $N_{o x}$, is calculated from Eq. 6.18. The voltage difference between threshold and midgap is related to the contribution 
of acceptor traps to the effective oxide charge. This voltage difference $V_{i t}$, is calculated from Eq. 6.19. The interface trap density, $N_{i t}$, is calculated from Eq. 6.20 with (+) for p-bulk Si.

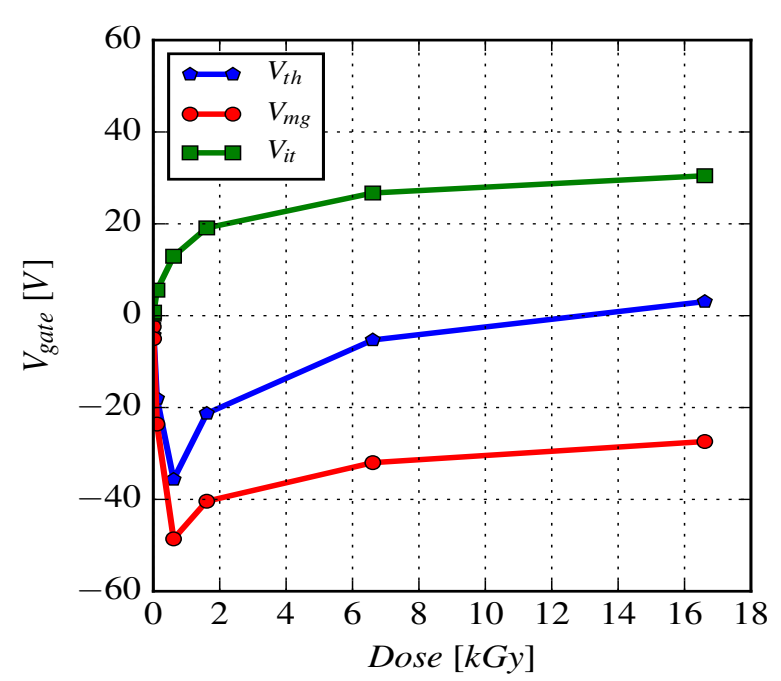

(a)

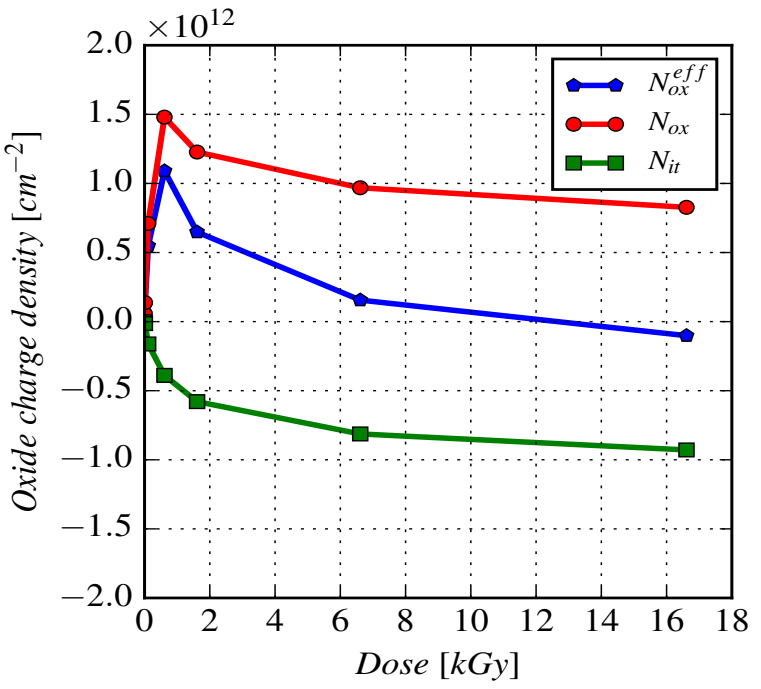

(b)

Figure 6.17: (a) Gate voltage and, (b) Oxide charge density as a function of X-ray dose of the n-MOSFET $<100>$ irradiated with $E_{\text {field }} \uparrow$.

The dose dependence of $V_{t h}, V_{m g}$ and $V_{i t}$ is presented in Fig. 6.17 (a) and the calculated $N_{o x}^{e f f}, N_{o x}$ and $N_{i t}$ in Fig. 6.17 (b). $V_{t h}$ shows a negative shift from $\approx-2.1 \mathrm{~V}$ to $\approx-35.7 \mathrm{~V}$ up to an X-ray dose of $610 \mathrm{~Gy}$ and positive shift from $\approx-35.7 \mathrm{~V}$ to $\approx 3.1 \mathrm{~V}$ for dose values of $610 \mathrm{~Gy}$ to $16.61 \mathrm{kGy}$.

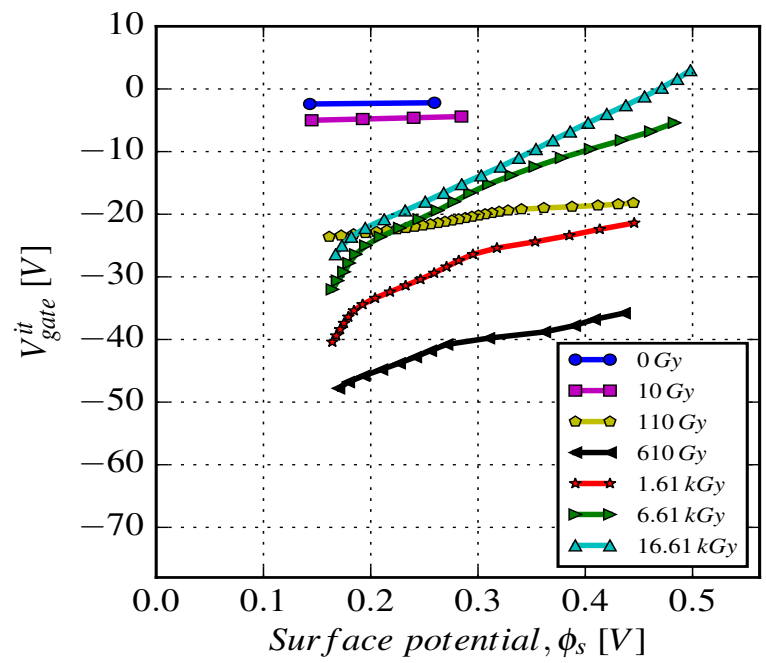

Figure 6.18: Gate voltage as a function of surface potential in the subthreshold region before and after X-ray irradiation of the n-MOSFET $<100>$ irradiated with $E_{\text {field }} \uparrow$.

The contribution of $V_{m g}$ to the net $V_{t h}$ shift is from $\approx-2.4 \mathrm{~V}$ to $\approx-48.6 \mathrm{~V}$ and from $\approx-48.6 \mathrm{~V}$ to $\approx$ $-27.4 \mathrm{~V}$. The contribution of $V_{i t}$ to the net $V_{t h}$ shift is from $\approx 0.3 \mathrm{~V}$ to $\approx 12.9 \mathrm{~V}$ and from $12.9 \mathrm{~V}$ to $30.5 \mathrm{~V}$. The change in $V_{t h}$ is explained by the subtractive effect of the charge contribution of interface traps and the 
fixed oxide charge to the net $V_{t h}$ shift. So far, $N_{o x}^{e f f}$ increases from $\approx 5.6 \cdot 10^{10} \mathrm{~cm}^{-2}$ to $\approx 1.1 \cdot 10^{12} \mathrm{~cm}^{-2}$ up to 610 Gy and then decreases to $\approx-1.0 \cdot 10^{11} \mathrm{~cm}^{-2}$ up to X-ray dose of $16.61 \mathrm{kGy}$. In particular, $N_{o x}$ increases from $\approx 5.7 \cdot 10^{10} \mathrm{~cm}^{-2}$ to $\approx 1.5 \cdot 10^{12} \mathrm{~cm}^{-2}$ and then decreases to $\approx 8.3 \cdot 10^{11} \mathrm{~cm}^{-2}$. The negative charge on the interface traps, $N_{i t}$, increases from $\approx 0.1 \cdot 10^{10} \mathrm{~cm}^{-2}$ to $\approx 9.3 \cdot 10^{11} \mathrm{~cm}^{-2}$ up to X-ray dose of $16.61 \mathrm{kGy}$. The $V_{\text {gate }}^{i t}\left(\phi_{s}\right)$ between midgap and threshold is determined after solving the non-linear equation $I_{d s}^{\text {ideal }}\left(\phi_{s}\right)-I_{d s}=0$. The solution gives the surface potential values, $\phi_{s}$, and the $V_{g a t e}^{i t}\left(\phi_{s}\right)$ as a function of X-ray dose is presented in Fig. 6.18. The slope of the $V_{\text {gate }}^{\text {it }}\left(\phi_{s}\right)$ curves increases as a function of dose due to the increase of interface traps.

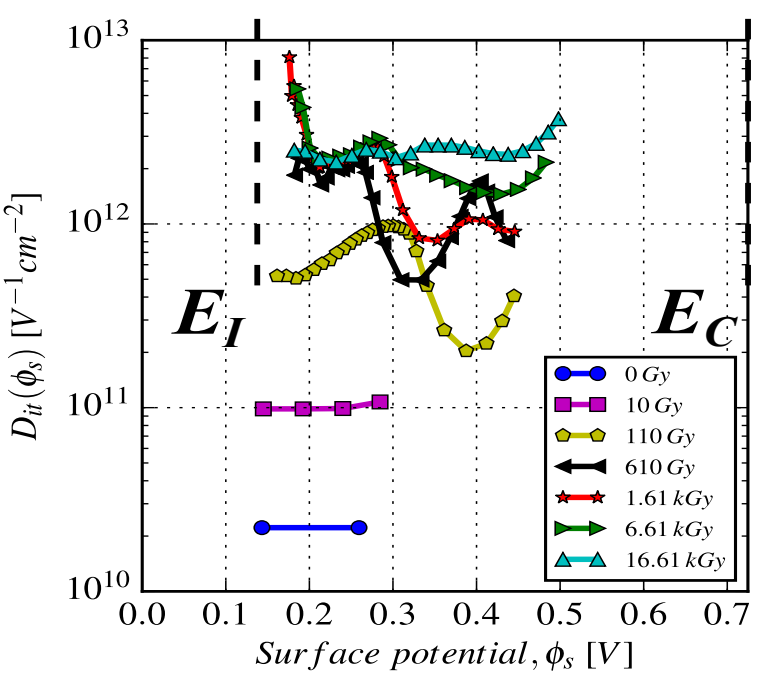

(a)

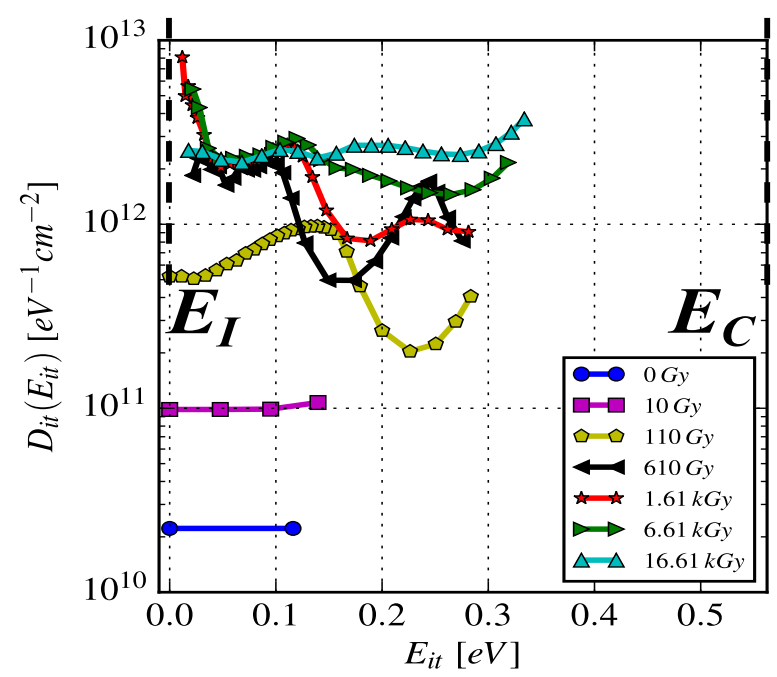

(b)

Figure 6.19: (a) $D_{i t}$ as a function of the surface potential and, (b) Energy in the Si bandgap before and after $\mathrm{X}$-ray irradiation of the n-MOSFET $<100>$ irradiated with $E_{\text {field }} \uparrow$.

The density of interface traps as a function of surface potential, $D_{i t}\left(\phi_{s}\right)$, is calculated from Eq. 6.17 and presented in Fig. 6.19 (a). With this method the energy distribution of interface traps (acceptor traps) between midgap, $\phi_{s}=\phi_{b}$ and threshold or strong inversion $\phi_{s} \geq 2 \phi_{b}$ has been extracted for the n-MOSFET. In Fig. 6.19 (b) is presented the energy distribution of interface traps, $D_{i t}\left(E_{i t}\right)$, where $E_{i t}=q_{0}\left(\phi_{s}-\phi_{b}\right)$ is calculated from the middle of the Si bandgap. The integrated $D_{i t}$ values, between midgap and threshold, correspond to the $\left|N_{i t}\right|$ values extracted for each X-ray dose, presented in Fig. 6.17 (b). The $D_{i t}$ in the midgap position increases from $\approx 2.0 \cdot 10^{10} \mathrm{eV}^{-1} \mathrm{~cm}^{-2}$ to $\approx 8.0 \cdot 10^{12} \mathrm{eV}^{-1} \mathrm{~cm}^{-2}$ up to $1.61 \mathrm{kGy}$ and then decreases to $\approx 2.0 \cdot 10^{12} \mathrm{eV}^{-1} \mathrm{~cm}^{-2}$ up to $16.61 \mathrm{kGy} \mathrm{X}$-ray dose. The energy position of the threshold voltage varies from $\approx 0.12$ to $\approx 0.33 \mathrm{eV}$ above midgap and the $D_{i t}$ increases from $\approx 2.0 \cdot 10^{10} \mathrm{eV}^{-1} \mathrm{~cm}^{-2}$ to $\approx 4.0 \cdot 10^{12} \mathrm{eV}^{-1} \mathrm{~cm}^{-2}$ up to X-ray dose of $16.61 \mathrm{kGy}$. The average value of $\bar{D}_{i t}$ in the energy range between midgap and threshold increases from $\approx 2 \cdot 10^{10} \mathrm{eV}^{-1} \mathrm{~cm}^{-2}$ to $\approx 2.6 \cdot 10^{12} \mathrm{eV}^{-1} \mathrm{~cm}^{-2}$ and is higher than the integrated interface trap density, $\left|N_{i t}\right|$. The energy distribution of interface traps from midgap $E_{I}$, towards the conduction band edge $E_{C}$, is constant for 0 and $10 \mathrm{~Gy}$, shows "peaks" for $110 \mathrm{~Gy}$ up to $1.61 \mathrm{kGy}$ and approximately is flattened for 6.61 and $16.61 \mathrm{kGy} \mathrm{X}$-ray dose. 


\subsubsection{The n-MOSFET irradiated with $E_{\text {field }}$ pointing from the $\mathrm{SiO}_{2}$ into the $\mathrm{Si}$}

Similar to the analysis presented in the previous section, the fixed oxide charge density, the interface trap density and the energy distribution of interface traps are extracted for the n-MOSFET $<100>$ irradiated with $E_{\text {field }}$ pointing $\downarrow$ from the $\mathrm{SiO}_{2}$ into the Si. In Fig. 6.20 (a) is presented the $I_{d s}\left(V_{\text {gate }}\right)$ in log scale as a function of X-ray dose in the subthreshold region.

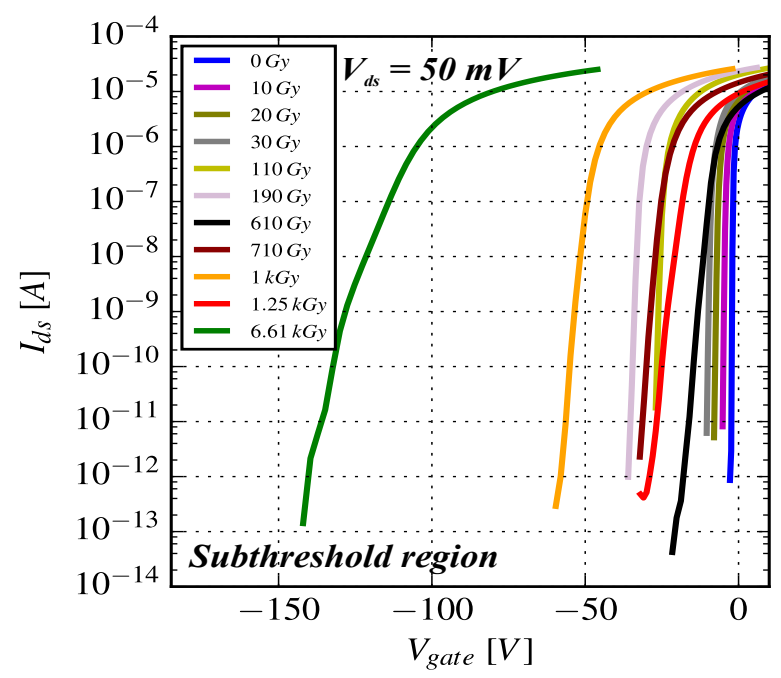

(a)

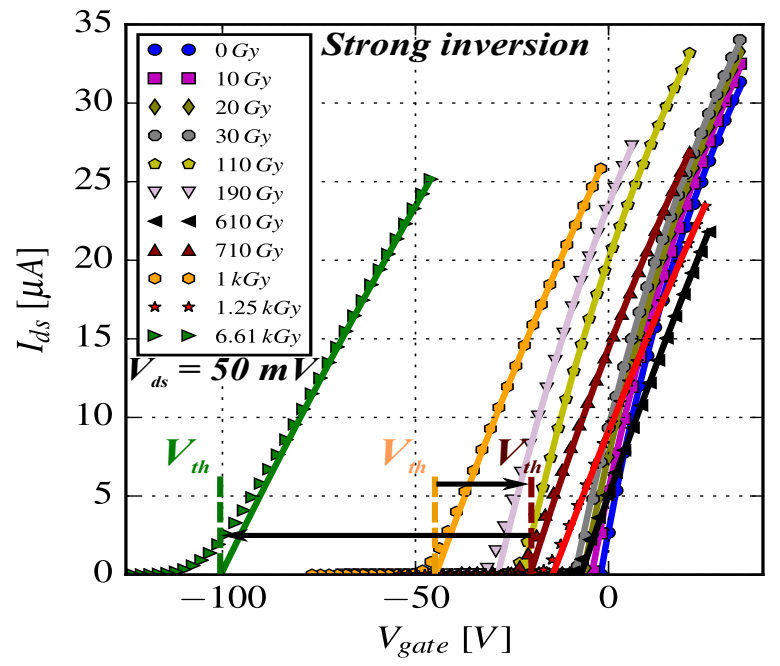

(b)

Figure 6.20: (a) $I_{d s}\left(V_{\text {gate }}\right)$ in the subthreshold region, (b) $I_{d s}\left(V_{\text {gate }}\right)$ in strong inversion and the parametrization model fit used to determine the threshold voltage, $V_{t h}$, before and after X-ray irradiation of the nMOSFET $<100>$ irradiated with $E_{\text {field }} \downarrow$.

As mentioned above for the n-MOSFET, the increase in "stretchout" as a function of X-ray dose of the subthreshold curves is caused by the increase of interface traps in the upper part of the Si bandgap. Using the midgap method, the midgap voltage, $V_{m g}$, is determined after the calculation of the midgap current, $I_{m g}$, for each subthreshold curve. The mobility $\mu_{0}$ values extracted in strong inversion, Fig. 6.20 (b), are used for the calculation of $I_{m g}$. As presented in Ch. $5, \mu_{0}$ decreases, so $I_{m g}$ decreases with increasing dose. The calculated $I_{m g}$ and extracted $V_{m g}$ values are presented in Table 6.4. The fixed oxide charge density, $N_{o x}$, is calculated from Eq. 6.18. The $V_{i t}$ "strechout" is related to the presence of acceptor traps between midgap and threshold and calculated from Eq. 6.19, where the interface trap density, $N_{i t}$, is calculated from Eq. 6.20 with (+) for p-bulk Si. The dose dependence of $V_{t h}, V_{m g}$ and $V_{i t}$ is presented in Fig. 6.21 (a) and the calculated $N_{o x}^{e f f}, N_{o x}$ and $N_{i t}$ in Fig. 6.21 (b). $V_{t h}$ shows a negative shift from $\approx-2.1 \mathrm{~V}$ to $\approx-28.7 \mathrm{~V}$ up to an X-ray dose of $190 \mathrm{~Gy}$ and a positive shift from $\approx-28.7 \mathrm{~V}$ to $\approx-7.1 \mathrm{~V}$ for a dose of 190 to $610 \mathrm{~Gy}$. Again a negative shift from $\approx-7.1 \mathrm{~V}$ to $\approx-44.8 \mathrm{~V}$ for a dose of $610 \mathrm{~Gy}$ to $1 \mathrm{kGy}$, a positive shift from $\approx-44.8 \mathrm{~V}$ to $\approx-14.5 \mathrm{~V}$ for 1 to $1.25 \mathrm{kGy}$ and negative shift from $\approx-14.5 \mathrm{~V}$ to $\approx-100.4 \mathrm{~V}$ for 1.25 to $6.61 \mathrm{kGy}$. The contribution of $V_{m g}$ to the net $V_{t h}$ shift is from $\approx-2.4 \mathrm{~V}$ to $\approx-35.4 \mathrm{~V}$, from $\approx-35.4 \mathrm{~V}$ to $\approx-16.8 \mathrm{~V}$, from $\approx-16.8 \mathrm{~V}$ to $\approx-57.0 \mathrm{~V}$, from $\approx-57.0 \mathrm{~V}$ to $\approx-27.4 \mathrm{~V}$ and from $\approx-27.4 \mathrm{~V}$ to $\approx-138.6 \mathrm{~V}$. The contribution of $V_{i t}$ to the net $V_{t h}$ shift is from $\approx 0.4 \mathrm{~V}$ to $\approx 6.7 \mathrm{~V}$, from $\approx 6.7 \mathrm{~V}$ to $\approx 9.7 \mathrm{~V}$, from $\approx 9.7 \mathrm{~V}$ to $\approx 12.2 \mathrm{~V}$ and from $\approx 12.2 \mathrm{~V}$ to $\approx 38.2 \mathrm{~V}$. 
Table 6.4: Charge separation results as a function of X-ray dose of the n-MOSFET $<100>$ irradiated with $E_{\text {field }} \downarrow$.

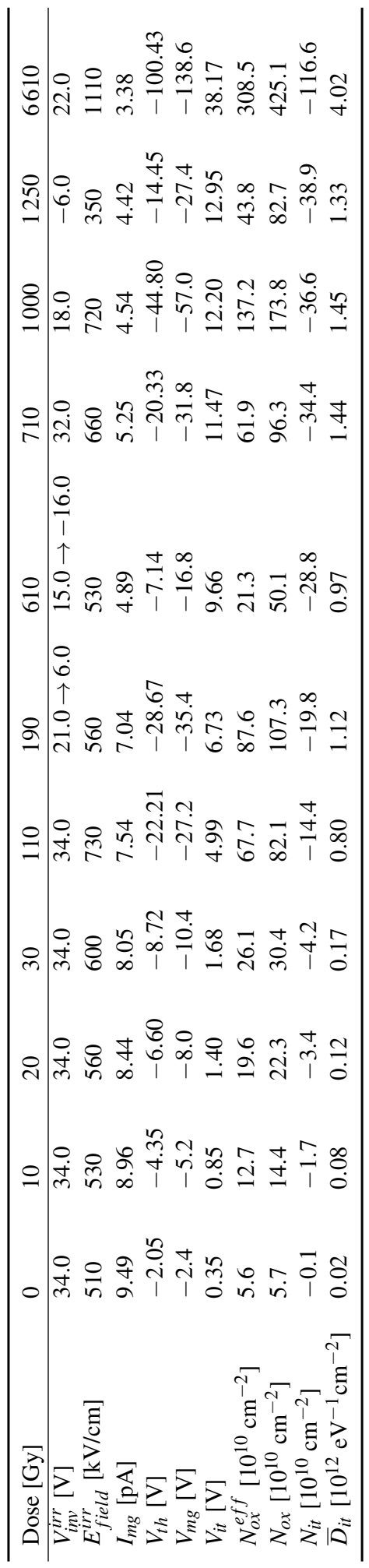




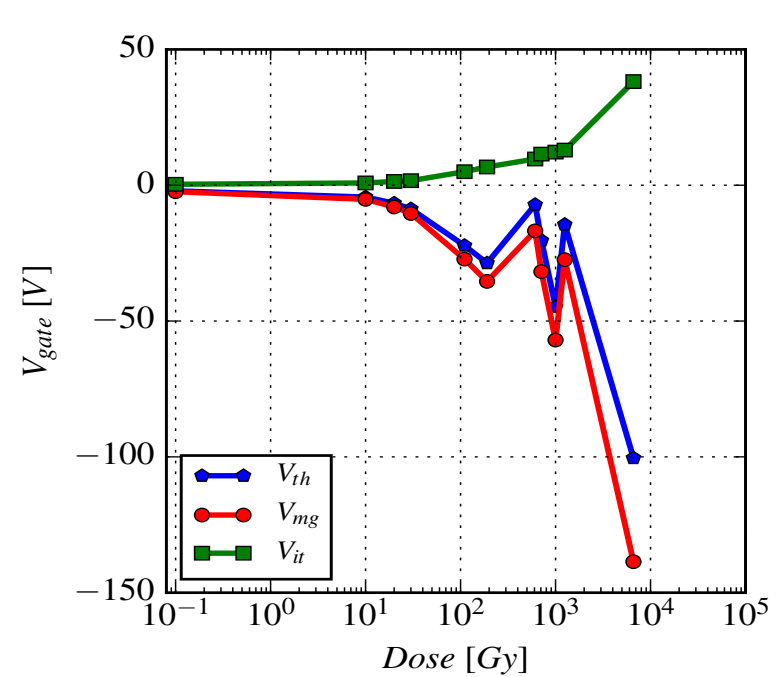

(a)

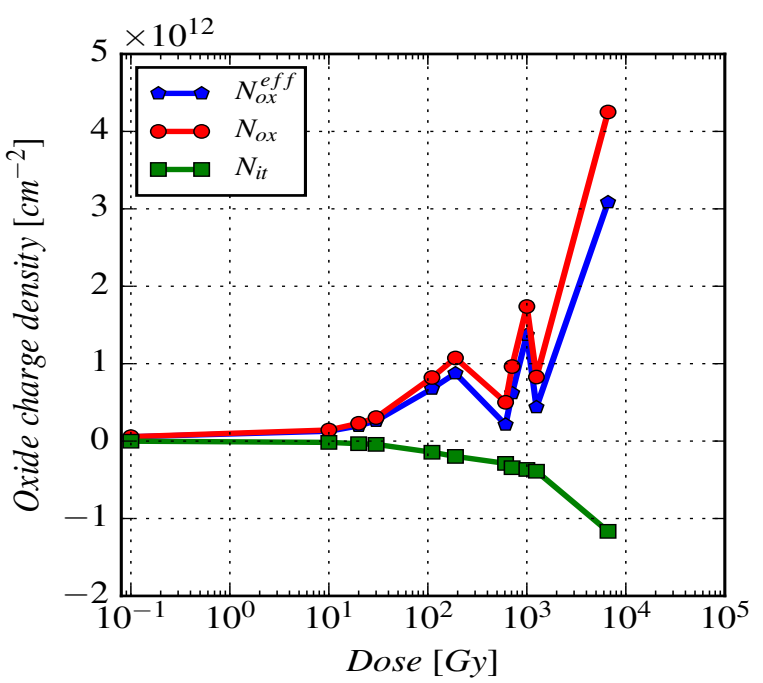

(b)

Figure 6.21: (a) Gate voltage and, (b) Oxide charge density as a function of X-ray dose of the n-MOSFET $<100>$ irradiated with $E_{\text {field }} \downarrow$.

As for the n-MOSFET irradiated with $E_{\text {field }} \uparrow$, the change in $V_{\text {th }}$ is explained by the subtractive effect of charge contribution of interface traps and fixed oxide charge to the net $V_{t h}$ shift. $N_{o x}^{e f f}$ increases from $\approx 5.6 \cdot 10^{10} \mathrm{~cm}^{-2}$ to $\approx 8.8 \cdot 10^{11} \mathrm{~cm}^{-2}$ up to $190 \mathrm{~Gy}$, decreases to $\approx 2.1 \cdot 10^{11} \mathrm{~cm}^{-2}$ up to $610 \mathrm{~Gy}$ and increases to $\approx 1.4 \cdot 10^{12} \mathrm{~cm}^{-2}$ up to $1 \mathrm{kGy} \mathrm{X}$-ray dose. Again decreases to $\approx 4.4 \cdot 10^{11} \mathrm{~cm}^{-2}$ up to $1.25 \mathrm{kGy}$ and increases to $\approx 3.1 \cdot 10^{12} \mathrm{~cm}^{-2}$ up to $6.61 \mathrm{kGy}$.

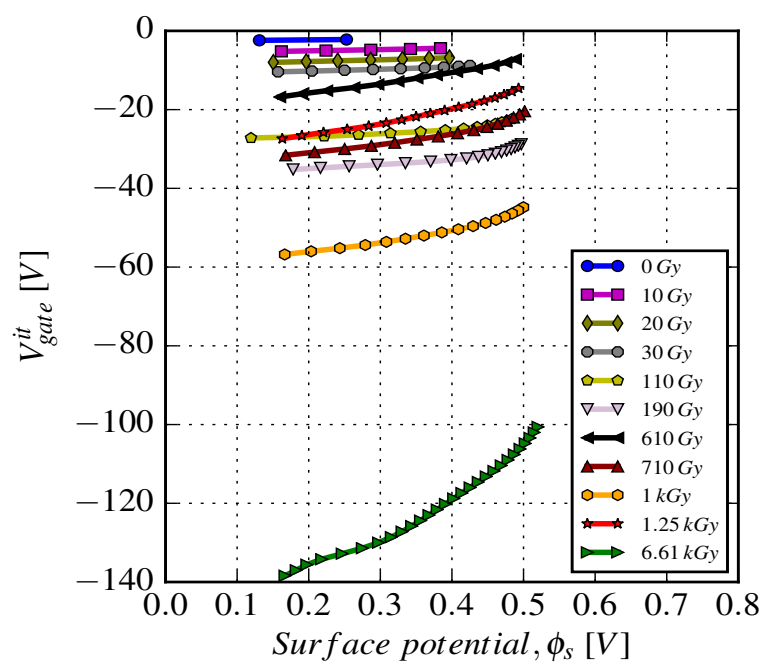

Figure 6.22: Gate voltage as a function of surface potential in the subthreshold region before and after X-ray irradiation of the n-MOSFET $<100>$ irradiated with $E_{\text {field }} \downarrow$.

In particular, $N_{o x}$ increases from $\approx 5.7 \cdot 10^{10} \mathrm{~cm}^{-2}$ to $\approx 1.1 \cdot 10^{12} \mathrm{~cm}^{-2}$, decreases to $\approx 5.0 \cdot 10^{11} \mathrm{~cm}^{-2}$, increases to $\approx 1.7 \cdot 10^{12} \mathrm{~cm}^{-2}$, decreases to $\approx 8.3 \cdot 10^{11} \mathrm{~cm}^{-2}$ and increases to $\approx 4.3 \cdot 10^{12} \mathrm{~cm}^{-2}$. The negative charge on the interface traps, $N_{i t}$, increases from $\approx 0.1 \cdot 10^{10} \mathrm{~cm}^{-2}$ to $\approx 1.2 \cdot 10^{12} \mathrm{~cm}^{-2}$. The $V_{\text {gate }}^{\text {it }}\left(\phi_{s}\right)$ between midgap and threshold is determined after solving the non-linear equation $I_{d s}^{\text {ideal }}\left(\phi_{s}\right)-I_{d s}=$ 
0 . The solution gives the surface potential values, $\phi_{s}$, and the $V_{\text {gate }}^{i t}\left(\phi_{s}\right)$ as a function of X-ray dose is presented in Fig. 6.22. The slope of the $V_{\text {gate }}^{i t}\left(\phi_{s}\right)$ curves increases as a function of dose due to the increase of interface traps. The density of interface traps as a function of surface potential, $D_{i t}\left(\phi_{s}\right)$, is calculated using Eq. 6.17 and presented in Fig. 6.23 (a). In addition, the energy distribution of interface traps, $D_{i t}\left(E_{i t}\right)$, where $E_{i t}=q_{0}\left(\phi_{s}-\phi_{b}\right)$ is calculated from the middle of the Si bandgap, is presented in Fig. 6.23 (b).

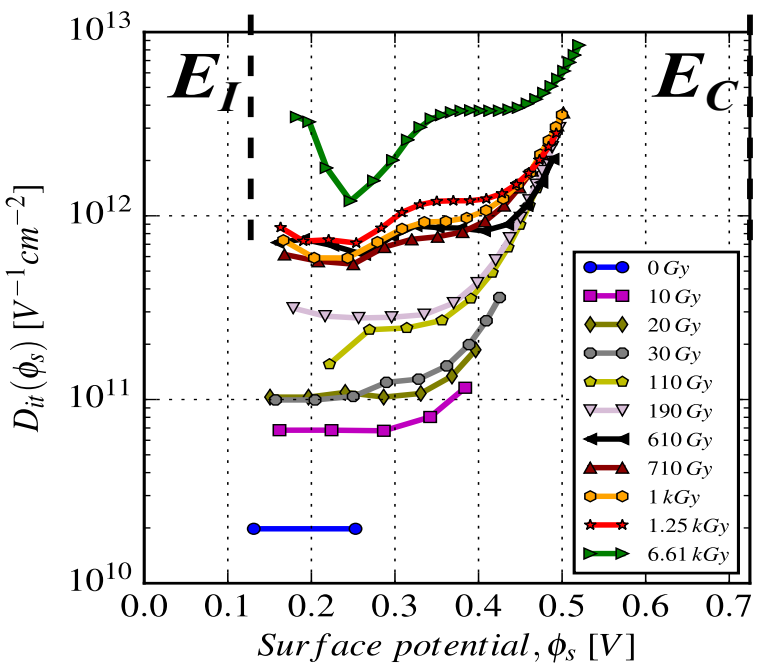

(a)

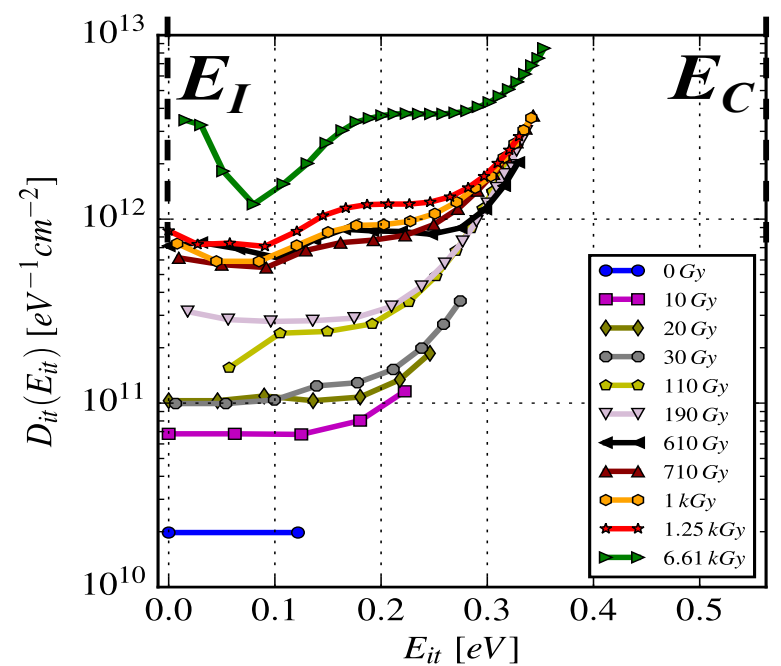

(b)

Figure 6.23: (a) $D_{i t}$ as a function of the surface potential and, (b) Energy in the Si bandgap before and after X-ray irradiation of the n-MOSFET $<100>$ irradiated with $E_{\text {field }} \downarrow$.

The $D_{i t}$ in the midgap position increases from $\approx 2.0 \cdot 10^{10} \mathrm{eV}^{-1} \mathrm{~cm}^{-2}$ to $\approx 1.2 \cdot 10^{12} \mathrm{eV}^{-1} \mathrm{~cm}^{-2}$ up to $6.61 \mathrm{kGy} \mathrm{X}$-ray dose. The energy position of the threshold has a variation from $\approx 0.12$ to $0.35 \mathrm{eV}$ above midgap and the $D_{i t}$ increases from $\approx 2.0 \cdot 10^{10} \mathrm{eV}^{-1} \mathrm{~cm}^{-2}$ to $\approx 10^{13} \mathrm{eV}^{-1} \mathrm{~cm}^{-2}$ up to X-ray dose of $6.61 \mathrm{kGy}$. The average value of $\bar{D}_{i t}$ in the energy range between midgap and threshold increases from $\approx 2.0 \cdot 10^{10} \mathrm{eV}^{-1} \mathrm{~cm}^{-2}$ to $\approx 4.0 \cdot 10^{12} \mathrm{eV}^{-1} \mathrm{~cm}^{-2}$ and is higher than the integrated interface trap density, $\left|N_{i t}\right|$. The results suggest a half "U" shaped distribution of traps, in the upper part of the Si bandgap with a "peak" at midgap after 610 Gy X-ray dose.

Discussion on the interface traps results As presented above and described in Refs. [173,174] the measurement of the electric field dependence of interface state build-up in thick oxides is complicated by the conflicting requirements to use a low dose rate to minimize the pertubation of the internal oxide field by space charge build-up and to use higher doses in order to minimize error in the measurement of $D_{i t}$. The electric field in the oxide is almost surely grossly pertubated for doses above $100 \mathrm{~Gy}$. Charge build-up strongly enhances the field at the $\mathrm{Si}_{-} \mathrm{SiO}_{2}$ interface under positive bias and tends to maintain it constant under negative bias [175].

The results for two oxide thicknesses, $250 \mathrm{~nm}$ and $700 \mathrm{~nm}$, indicate that it is not the properties of the $\mathrm{Si}_{-} \mathrm{SiO}_{2}$ interface alone that determine the build-up of interface state, a bulk effect must also be present. A possible mechanism is a variation in interface state build-up with strain at the interface that is in turn a function 
of oxide thickness [173]. To obtain these results I-V curves were recorded using gate voltage ramp rate $\approx 1 \mathrm{~V} / \mathrm{s}$. The $D_{i t}$ distributions have been extracted with a slow ramp, approaching the "true" interface state distributions. It is probable that prompt interface state build-up occurs in gate oxides but is usually masked by the two stage process $[90,176]$. The dominance of one build-up mechanism over the other is probably controlled largely by the oxide thickness. At applied fields below about $1 \mathrm{MV} / \mathrm{cm}$, interface state build-up in thick oxides takes place primarily. The strong effect under negative bias suggests that the $E_{\text {field }}$ at the $\mathrm{Si}-\mathrm{SiO}_{2}$ interface is particularly important to $N_{i t}$ build-up, and consequently the prompt $N_{i t}$ build-up may result primarily from local interactions near the interface rather than in the oxide bulk [174]. The prompt interface states may be caused by either a bulk process such as bond-breaking or lattice distortion leading to interface strain and consequential to $N_{i t}$, or by direct creation of states near the $\mathrm{Si}_{-} \mathrm{SiO}_{2}$. So far, important similarities have been observed for the two different orientations $<100>$ and $<111>$ irradiated up to $\approx 17 \mathrm{kGy}$ Xray dose. In a short list: i) all samples after irradiation exhibit a characteristic peak above the midgap, ii) all samples exhibit a peak below midgap at approximately the same energy position and iii) the peak below the midgap seems to be more stable than the peak above the midgap, similar to Ref. [177].

\subsection{Summary}

In this chapter an effort is made to separate the contribution of the oxide charge density and interface trap density to the effective oxide charge density determined in $\mathrm{Ch}$. 5. The method used is based on the Brews charge sheet model for long channel MOSFETs. It is only sensitive to interface traps in parts of the bandgap below midgap for p-MOSFETs and above midgap for n-MOSFETs. It is found that the interface trap density increases with X-ray dose for p-MOSFETs and n-MOSFETs and both directions of the electric field during irradiation in a similar way. In this chapter many assumptions have been made and further work is required to validate them. 


\section{Gate Controlled Diode measurements on MOSFETs}

A MOSFET with source floating or drain floating or source connected to drain is similar to a Gate Controlled Diode structure $[178,179]$. In the present chapter, measurements have been performed on $\mathrm{p}$ - and n-MOSFETs at room temperature before and after irradiation. It is presented that the surface generation current, $I_{\text {surf }}$, and the density of the generation recombination centers at the $\mathrm{Si}_{-} \mathrm{SiO}_{2}$ interface can be obtained from the gate controlled diode measurement on MOSFETs.

\subsection{Surface current}

As presented in Refs. $[178,180,181]$ in a GCD structure, the p-n junction reverse current may consist of one or more of the following three components depending on the gate voltage: (i) the current which corresponds to generation within the depletion region of the metallurgical junction $I_{m j}$, (ii) the current which corresponds to generation within the depletion region of the field induced junction $I_{f i j}$, and (iii) the current which corresponds to the surface generation component $I_{\text {surf }}$. Explanations of the above statements is suggested in Figs. 7.1 and 7.2. At room temperature the reverse current of p-n junctions is due to electron-hole pairs generated through the action of Shockley-Read-Hall type centers within the depletion region [182-184]. The

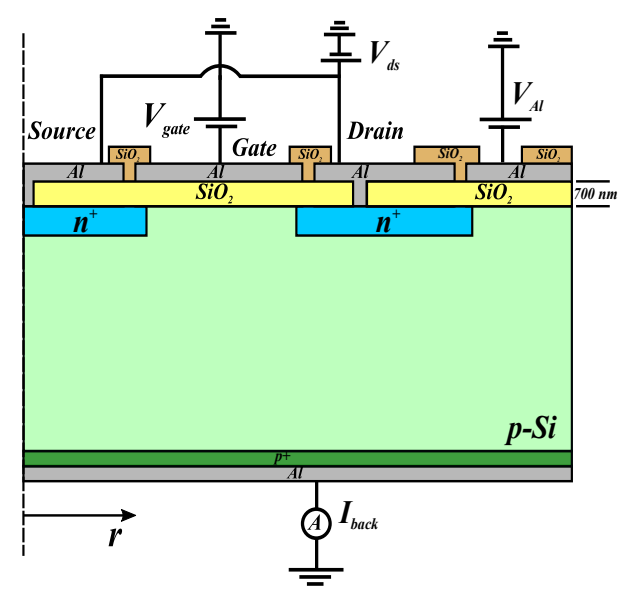

(a)

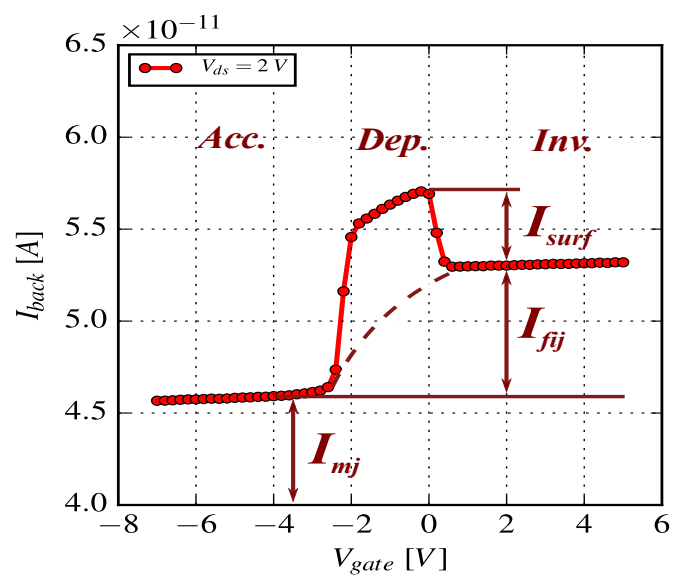

(b)

Figure 7.1: (a) Bias configuration with source connected to drain under test for the gate controlled measurement, and (b) Typical gate controlled diode characteristics $I_{\text {back }}$ as a function of $V_{\text {gate }}$ of the non-irradiated n-MOSFET <100>. 
magnitude of the reverse current depends on the total number of such centers included within the junction depletion region. For the n-MOSFET circular design shown in Fig. 7.1 (a), an external voltage $V_{A l}=-25 \mathrm{~V}$ is necessary in order to form a hole accumulation layer at the edge of the depletion region.

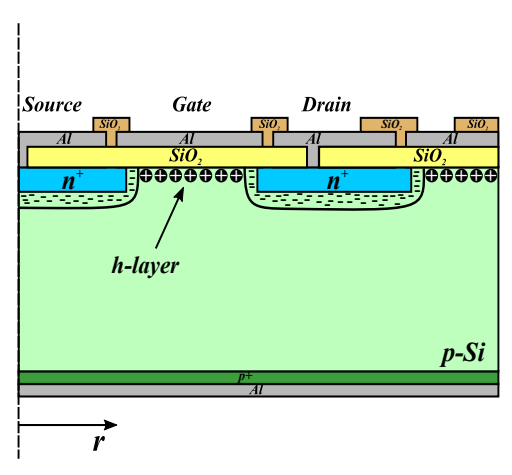

(a)

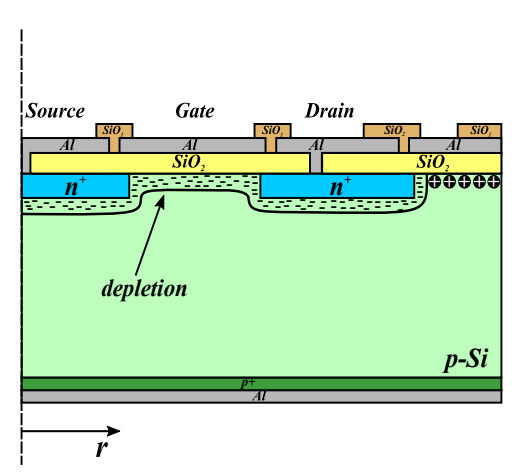

(c)

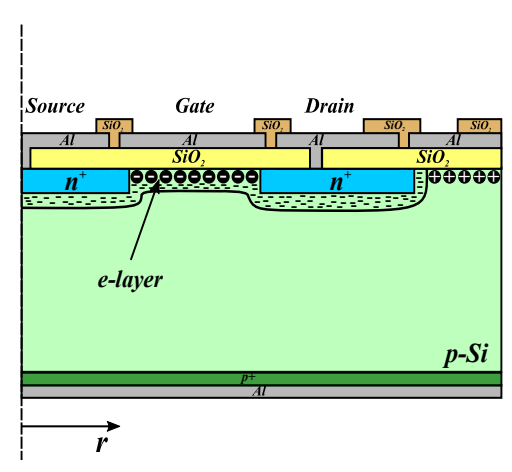

(e)

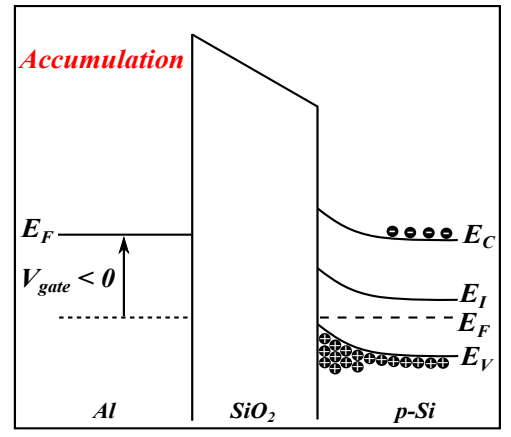

(b)

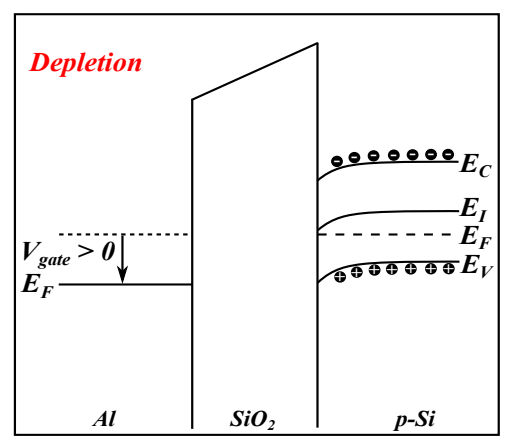

(d)

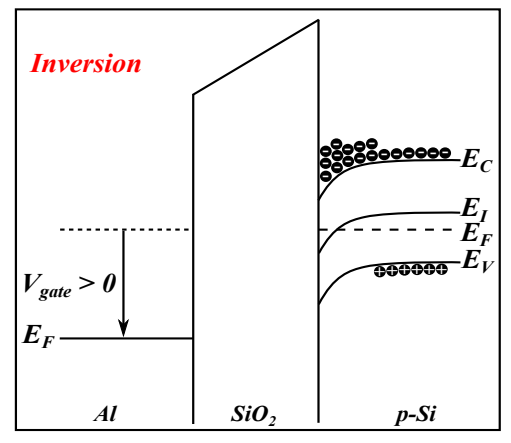

(f)

Figure 7.2: Cross section and Energy band diagram of the n-MOSFET $<100>$ in accumulation (a) and (b), depletion (c) and (d), inversion (e) and (f) respectively.

The hole accumulation layer prevents the surface current measurement from edge leakage current due to the junction between the p-Si substrate and the $\mathrm{SiO}_{2}$. When the surface under the gate is accumulated, 
Figs. 7.2 (a) and (b), only those centers which are within the depletion region of the metallurgical p-n junction contribute to the generation current. When the surface under the gate is inverted, Figs. 7.2 (e) and (f), centers within the depletion region of the field induced junction between the inversion layer and underlying substrate also contribute to the total generation current which is therefore larger than in the first case. When the surface is depleted, Figs. 7.2 (c) and (d), centers at the $\mathrm{Si}_{-} \mathrm{SiO}_{2}$ interface provide another contribution to the total generation current resulting in a peak in the reverse current vs. gate voltage characteristics. Such surface generation recombination centers or fast surface states can be charged and discharged. The contribution to the generation current associated with the surface depletion region is related to the width of this region $x_{d}$. When the surface is depleted, $x_{d}$ and hence this current component increases with increasing gate voltage $V_{\text {gate }}$, as indicated by the dotted line in Fig. 7.1 (b). When the surface is inverted, $x_{d}$ reaches its maximum value and there is no further increase in this current component. So far, the generation current may consist of one or more of the following three components depending on the nature of the surface space charge region [180],

$$
\begin{gathered}
I_{m j}=q_{0} U_{m j} W A_{m j} \\
I_{f i j}=q_{0} U_{f i j} x_{d_{\max }} A_{s} \\
I_{\text {surf }}=q_{0} U_{s} A_{s}
\end{gathered}
$$

where $U_{m j}$ and $U_{f i j}$ are the carrier generation rates per unit volume in the depletion regions of the metallurgical and field induced junctions, respectively. $U_{s}$ is the carrier generation rate per unit area at the $\mathrm{Si}_{-} \mathrm{SiO}_{2}$ interface, and $\mathrm{W}$ and $x_{d_{\max }}$ are the depletion region widths of the metallurgical and field induced junctions. $A_{m j}$ is the area of the metallurgical junction, and $A_{s}$ is the area of the substrate under the gate. It is evident from Eqs. 7.1 and 7.2 that whereas both bulk generation components should depend on the magnitude of the reverse bias voltage through $\mathrm{W}$ and $x_{d_{\max }}$. However the surface generation current, Eq. 7.3, is independent of the reverse bias [180].

\subsubsection{Before $X$-ray irradiation}

The gate controlled diode measurement on MOSFETs can be carried out with the source floating, reverse voltage $V_{d}$ applied on the drain, drain floating with reverse voltage $V_{s}$ applied to the source or the source connected to the drain with the reverse voltage $V_{d s}$ applied to source and drain $[178,180]$. The $V_{\text {gate }}$ for n-MOSFET is increased from a negative voltage (which corresponds to surface accumulation) to a positive voltage (which corresponds to strong inversion), while the drain (or the source or both the drain and source) is kept at a constant positive voltage and the current is measured from the backside $I_{\text {back }}$. The ramp rate of $V_{\text {gate }}$ should be low so the MOSFET is under steady-state conditions $[178,185]$. For the bias configuration (source floating) shown in Fig. 7.3 (a), when a positive voltage $\left(V_{d}>0\right)$ is applied to the drain, the p-n junction between the substrate and the drain is reverse biased. To describe this steady state situation the 
single Fermi level in equilibrium is replaced by the quasi-Fermi level $E_{f n}$ for electrons and the quasi-Fermi level $E_{f p}$ for holes, and the difference is

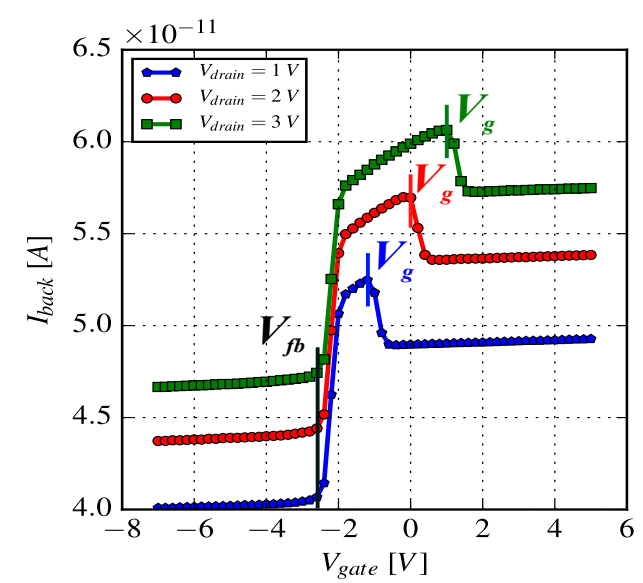

(a)

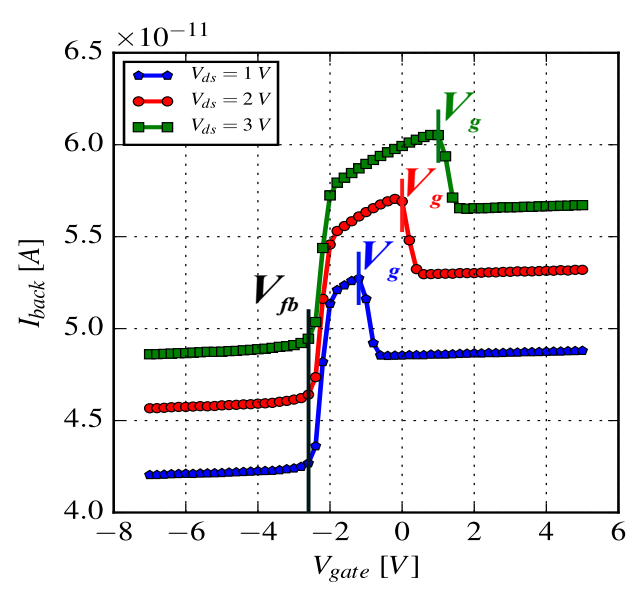

(b)

Figure 7.3: $I_{\text {back }}$ as a function of $V_{\text {gate }}$ before irradiation of the n-MOSFET $<100>$ (a) with source floating, reverse voltage $V_{d}$ on drain, and (b) with source connected to drain, reverse voltage $V_{d s}$.

$$
E_{f p}-E_{f n}=q_{0} V_{d}
$$

The quasi-Fermi level for electrons at the surface of the substrate under the gate is lowered by an amount $q_{0} V_{d}$ relative to the bulk Fermi level in the substrate. At the onset of weak inversion (midgap), the intrinsic level coincides with the quasi-Fermi level for electrons, so the corresponding surface potential is

$$
\phi_{s}=V_{d}+\phi_{b}
$$

where $q_{0} \phi_{b}$ is the energy difference between the intrinsic and the bulk Fermi level. The gate voltage at the onset of weak inversion can be written as

$$
V_{g}=V_{f b}+V_{d}+\phi_{b}+\frac{\sqrt{2 \varepsilon_{S i} \varepsilon_{0} q_{0} N_{d} 2 \phi_{b}}}{C_{o x}}
$$

where $V_{f b}$ is the flatband voltage of the MOS structure, $\varepsilon_{S i}$ the Si permittivity, $N_{d}$ the bulk doping of the substrate and $C_{o x}$ the oxide capacitance per unit area. The voltages $V_{f b}$ and $V_{g}$ are indicated in Figs. 7.3 (a) and (b) for $I_{\text {back }}\left(V_{\text {gate }}\right)$ measurements for different values of $V_{d}$ and $V_{d s}$. For a sufficiently negative $V_{\text {gate }}$ the surface is in accumulation and only the centers within the depletion region of the metallurgical drain/substrate p-n junction contribute to the generation current $I_{d m j}$. When $V_{\text {gate }}$ is sufficiently positive the surface is in inversion and the source is connected to drain internally by the inversion layer. In this case, the centers within the depletion regions of the metallurgical source/substrate $p-n$ junction and the field induced p-n junction also contribute to the generation current. So far, the reverse current is the sum of the currents $I_{d m j}, I_{s m j}$ and $I_{f i j}$ when the surface is strongly inverted, where $I_{s m j}$ and $I_{f i j}$ are the generation currents of 
the metallurgical source/substrate junction and the field induced junction respectively. When the surface is depleted, the generation-recombination centers at the $\mathrm{Si}_{-} \mathrm{SiO}_{2}$ add the surface current component $I_{\text {surf }}$. As a result, the maximum reverse current is observed at a $V_{\text {gate }}$ for which the silicon surface under the gate is depleted. However as the metallurgical source/substrate and drain/substrate junctions are not identical, the current $I_{d m j}$ of the drain/substrate junction is not equal to the current $I_{s m j}$ of the source/drain junction. Hence the current $I_{f i j}$ cannot be determined simply from the gate controlled measurement with source floating. This problem can be solved with the measurement where the source is connected to drain [178]. In this case, when the surface is accumulated both the source/substrate and drain/substrate junctions contribute to the reverse current $I_{a c c}$ or $I_{m j}$ where,

$$
I_{a c c}=I_{m j}=I_{s m j}+I_{d m j}
$$

and when the surface is strongly inverted the reverse current $I_{i n v}$ is defined as,

$$
I_{i n v}=I_{s m j}+I_{d m j}+I_{f i j}
$$

According to Eqs. 7.7 and 7.8, $I_{f i j}$ can be determined from $I_{f i j}=I_{i n v}-I_{a c c}$. Similar to the bulk G-R centers, interface traps at the $\mathrm{Si}_{-} \mathrm{SiO}_{2}$ interface can also act as G-R centers and they are responsible for the observed surface generation current $I_{\text {surf }}$. As shown in Refs. [178, 181], only the interface traps energy levels $E_{i t}$ close to the midgap $E_{I}$ (i.e. $E_{i t} \approx E_{I}$ ) are efficient surface G-R centers. When the surface under the gate is depleted, the surface generation current component can be written as

$$
I_{\text {surf }}=\frac{1}{2} q_{0} n_{i} \sigma v_{t h} N_{i t} A_{s}
$$

where $v_{t h}$ is the thermal velocity, $N_{i t}$ the density of interface traps with energy states near midgap and $\sigma$ is their capture cross section which is assumed to be the same for electrons and holes. When the $I_{\text {surf }}$ is extracted the surface G-R centers can be obtained from Eq. 7.9.

\subsubsection{After X-ray irradiation}

Surface current measurements after X-ray irradiation have been performed on the n-MOSFET $<100>$ irradiated with $E_{\text {field }} \uparrow$. As shown in Fig. 7.4 (a), the $I_{\text {back }}\left(V_{\text {gate }}\right)$ with drain-source voltage $V_{d s}=10 \mathrm{~V}$ has been measured as a function of X-ray dose. The value of $V_{f b}$ shows a negative shift up to $610 \mathrm{~Gy} \mathrm{X}$-ray dose, due to the increase of the oxide charge density. For the higher doses up to $16.61 \mathrm{kGy}$ a positive shift is observed, due to the decrease of the oxide charge, in agreement with the results presented in Chs. 5 and 6. However the surface current $I_{\text {surf }}$ increases as a function of X-ray dose, independent of the $V_{f b}$ shift, due to the increase of the interface trap density. For a cross check of the reverse applied voltage on drain-source $V_{d s}$, or drain voltage $V_{d}$, several reverse voltages have been applied from 1.0 up to $10 \mathrm{~V}$, as shown in Fig. 7.4 (b). As the reverse voltage increases, the weak inversion shows a positive shift, as described by Eq. 7.6. In addition, the leakage current shows a small increase with the reverse voltage and is visible in the accumulation and 
inversion conditions. $V_{f b}$ is independent of the reverse voltage and depends only on the oxide charge density. So far, $V_{d s}$ was chosen $10 \mathrm{~V}$, as the $I_{\text {surf }}$ is approximately saturated, Fig. 7.4 (b). However, there are small differences between the three possible bias configurations, reverse voltage $V_{d s}$ on drain-source, reverse voltage $V_{d}$ on drain with source floating and reverse voltage $V_{\text {source }}$ on source with drain floating.

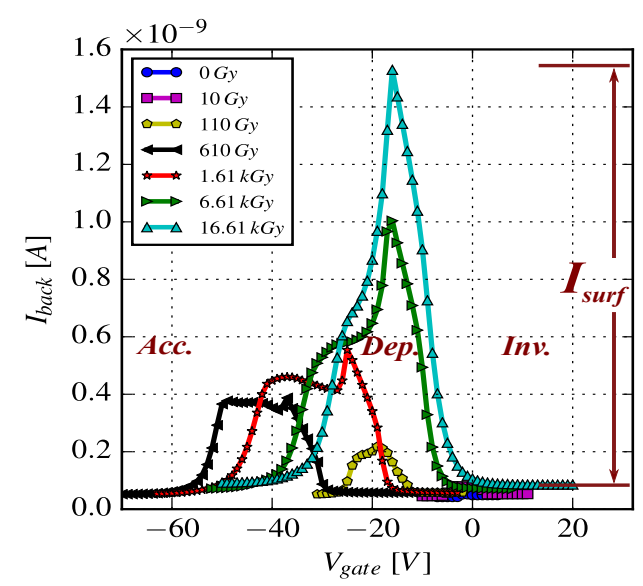

(a)

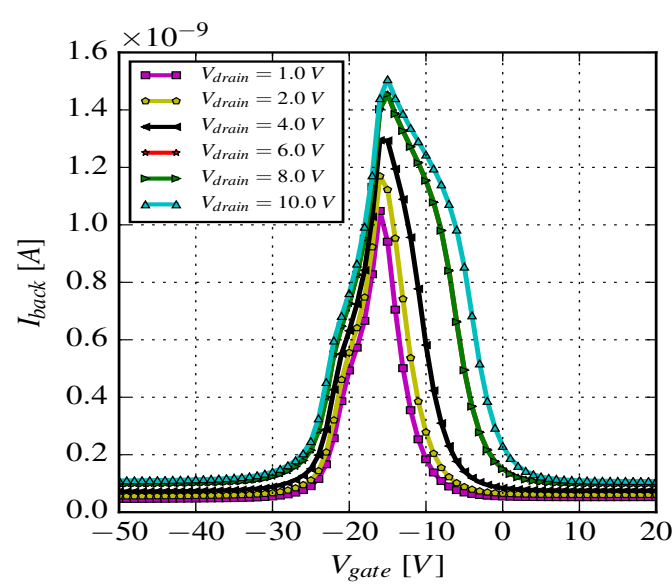

(b)

Figure 7.4: $I_{\text {back }}$ as a function of $V_{\text {gate }}$ of n-MOSFET <100> irradiated with $E_{\text {field }} \uparrow$ (a) with source connected to drain, reverse voltage $V_{d s}=10 \mathrm{~V}$, as a function of X-ray dose, and (b) with source floating, reverse voltage $V_{d}$ on drain after $16.61 \mathrm{kGy} \mathrm{X}$-ray dose.

As presented in Fig. 7.5 (a) for the n-MOSFET $<100>$ irradiated with $E_{\text {field }} \uparrow$ after $16.61 \mathrm{kGy}$ X-ray dose, the measurement with reverse voltage $V_{\text {source }}$ shows a lower leakage current in accumulation due to the smaller area of the metallurgical p-n junction. The measurement with drain-source reverse voltage $V_{d s}$ shows a higher surface current, due to the higher depleted surface area below the gate compared to the other two bias configurations. Also the differences in the depleted surface area, are shown in the differences of $V_{f b}$ which is influenced from the oxide charge density. As expected, no differences are observed in the weak inversion voltage, as the reverse voltage of $10 \mathrm{~V}$ is the same for the three bias configurations.

In order to overcome the problem with the non-depleted surface, the measurement with reverse voltage on drain-source $V_{d s}$ has been chosen for the extraction of the surface current. The extracted parameters as a function of X-ray dose and electric field during irradiation $E_{\text {field }}^{\text {irr }}$ are presented in Table 7.1.

Table 7.1: Surface current density, $J_{\text {surf }}$, as a function of X-ray dose of the n-MOSFET <100> irradiated with $E_{\text {field }} \uparrow$.

\begin{tabular}{llllllll}
\hline Dose $[\mathrm{Gy}]$ & 0 & 10 & 110 & 610 & 1610 & 6610 & 16610 \\
\hline$E_{\text {field }}^{\text {irr }}[\mathrm{kV} / \mathrm{cm}]$ & -500 & -500 & -490 & -500 & -500 & -460 & -440 \\
$I_{\text {surf }}[\mathrm{nA}]$ & 0.003 & 0.02 & 0.14 & 0.38 & 0.54 & 0.97 & 1.42 \\
$J_{\text {surf }}\left[\mathrm{nA} / \mathrm{cm}^{2}\right]$ & 0.9 & 6 & 40 & 110 & 150 & 270 & 400 \\
\hline
\end{tabular}

The surface current density $J_{\text {surf }}=I_{\text {surf }} / A_{s}$ increases to $0.4 \mu \mathrm{A} / \mathrm{cm}^{2}$ up to $16.61 \mathrm{kGy}$ X-ray dose due to the increase of interface trap density. An attempt to present the contribution of the interface traps to the surface current is shown in Fig. 7.5 (b). The measurement of the surface current $I_{\text {back }}\left(V_{\text {gate }}\right)$ with reverse voltage on drain, $V_{d}$, is plotted together with the surface potential $\phi_{s}\left(V_{\text {gate }}\right)$ extracted with the subthreshold current 


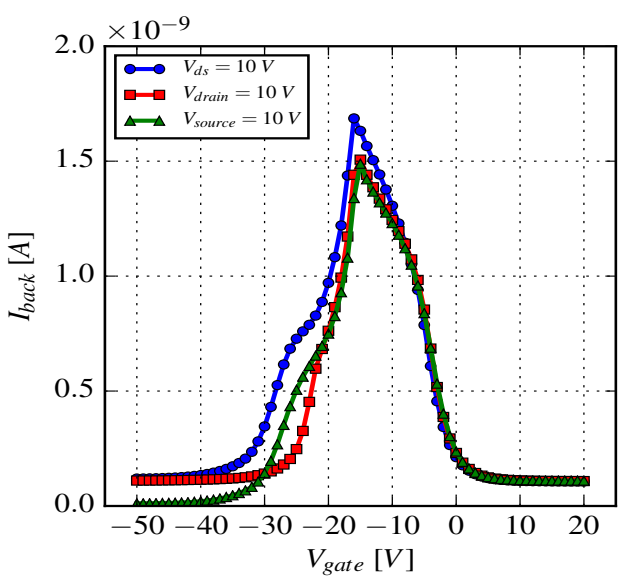

(a)

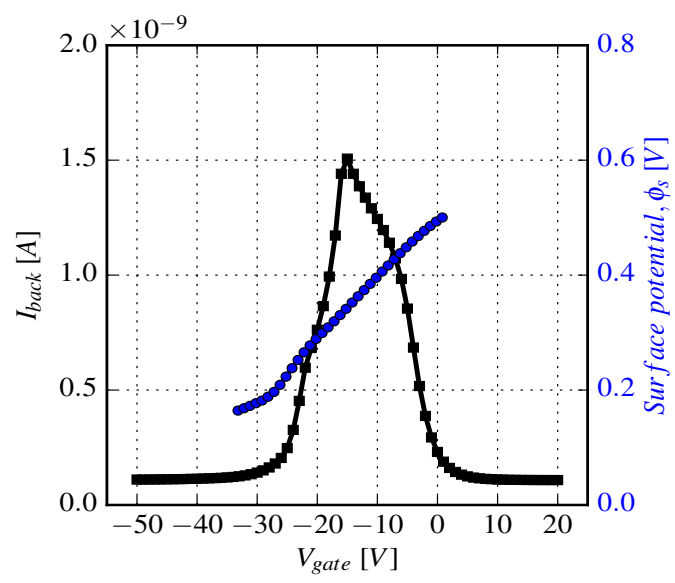

(b)

Figure 7.5: $I_{\text {back }}$ as a function of $V_{\text {gate }}$ of n-MOSFET $<100>$ irradiated with $E_{\text {field }} \uparrow$ after $16.61 \mathrm{kGy}$ X-ray dose (a) for the three possible bias configurations and (b) with source floating, reverse voltage $V_{d}=10 \mathrm{~V}$ on drain, and surface potential $\phi_{s}$ as a function of $V_{\text {gate }}$ extracted with the subthreshold current technique.

technique at $16.61 \mathrm{kGy} \mathrm{X}$-ray dose. The $I_{\text {back }}$ starts to increase due to $I_{\text {surf }}$ above $V_{f b}$ and the $\phi_{s}\left(V_{\text {gate }}\right)$ starts to increase from midgap to strong inversion as $V_{\text {gate }}$ increases. The small differences in gate voltage region $V_{\text {gate }}$, between the contribution of $I_{\text {surf }}$ to the $I_{\text {back }}$ and the increase of $\phi_{s}$ are related to the difference of the measurement techniques. The $\phi_{S}\left(V_{\text {gate }}\right)$ has been extracted from the drain-source current measurement $I_{d s}\left(V_{\text {gate }}\right)$. Similar the surface current measurement has been performed also for the n-MOSFET $<100>$ irradiated with $E_{\text {field }} \downarrow$ after $1.25 \mathrm{kGy}$ X-ray dose.

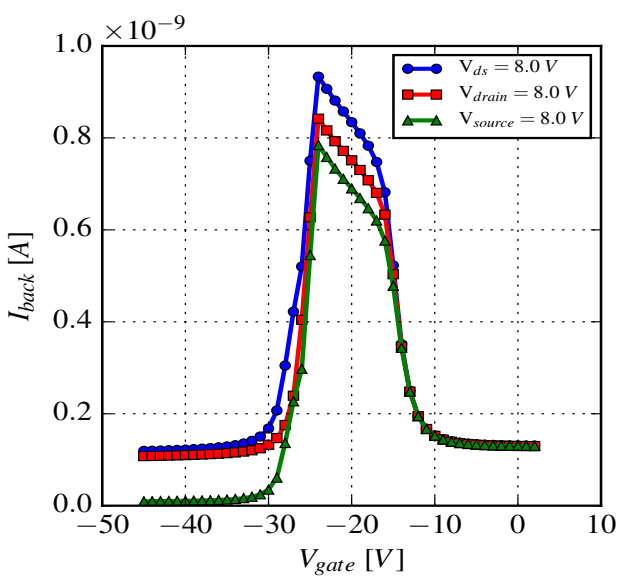

(a)

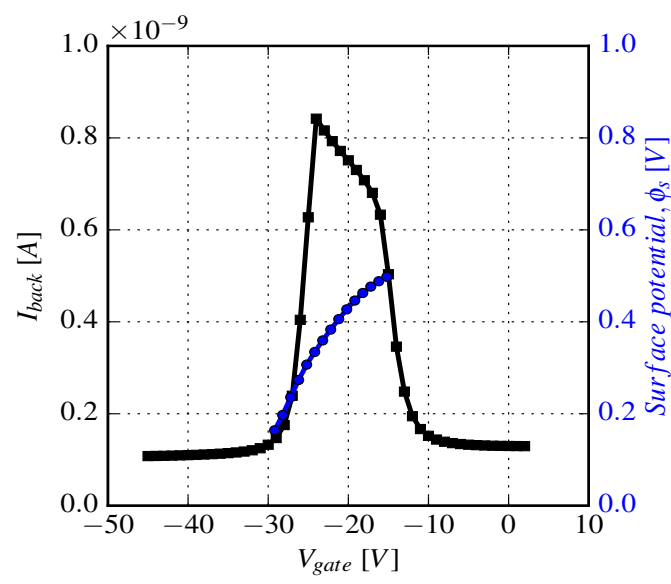

(b)

Figure 7.6: $I_{\text {back }}$ as a function of $V_{\text {gate }}$ of n-MOSFET $<100>$ irradiated with $E_{\text {field }} \downarrow$ after 1.25 kGy X-ray dose (a) for the three possible bias configurations and (b) with source floating, reverse voltage $V_{d}=8 \mathrm{~V}$ on drain, and $\phi_{s}$ as a function of $V_{\text {gate }}$ extracted with the subthreshold current technique.

As presented in Fig. 7.6 (a), the measurement with reverse voltage $V_{\text {source }}$ shows a lower leakage current in accumulation, the measurement with drain-source reverse voltage $V_{d s}$ shows a higher surface current and 
differences are found for $V_{f b}$. No differences are observed in the weak inversion voltage, as the reverse voltage of $8 \mathrm{~V}$ is the same for the three bias configurations. In Fig. 7.6 (b) is presented the contribution of the interface traps to the surface current. The $I_{b a c k}$ starts to increase due to $I_{\text {surf }}$ after $V_{f b}$ and the $\phi_{s}\left(V_{\text {gate }}\right)$ starts to increase from midgap to strong inversion as the $V_{\text {gate }}$ increases. The contribution of $I_{\text {surf }}$ to $I_{\text {back }}$ and the increase of $\phi_{s}$ is presented approximately in the same gate voltage region $V_{\text {gate }}$.

Measurements on p-MOSFET Surface current measurements have also been performed on the p-MOSFET circular design. For the p-MOSFET no external voltage is necessary, in order to form an accumulation layer and prevent the measurement from edge leakage current. There is no junction formed between the n-Si and the $\mathrm{SiO}_{2}$ at least for low X-ray doses. As shown in Figs. 7.7 (a) and Figs. 7.7 (b) for the p-MOSFET, $V_{\text {gate }}$ is changed from a positive voltage (which corresponds to surface accumulation) to a negative voltage (which corresponds to strong inversion), while the drain (or the source or both the drain and source) is kept at a constant negative voltage and the current is measured from the backside $I_{\text {back }}$. The relevant charge layers below the gate in accumulation, depletion and inversion and the corresponding band bending before irradiation are presented in Fig. 7.8.

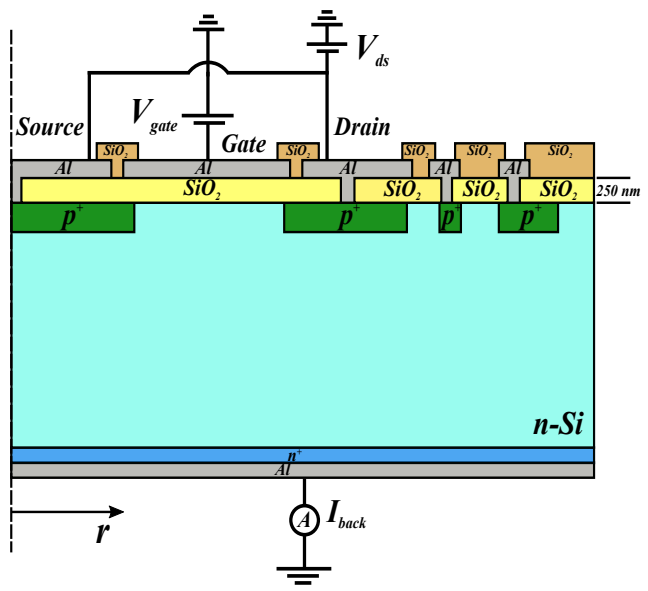

(a)

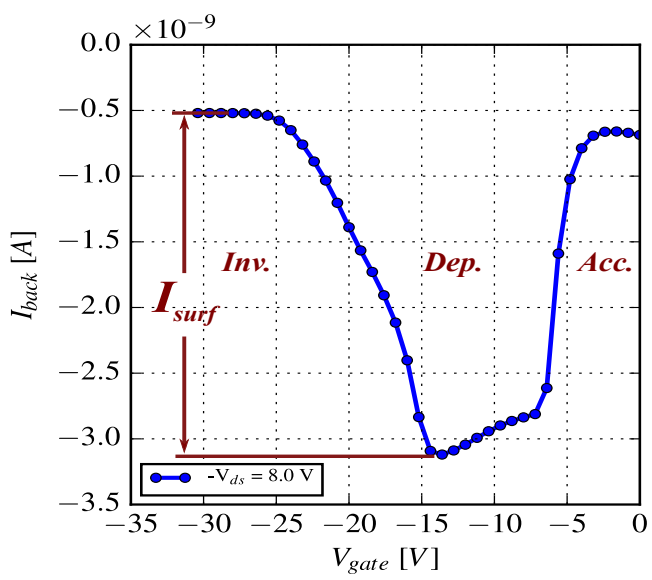

(b)

Figure 7.7: (a) Bias configuration with source connected to drain under test for the gate controlled measurement, and (b) Typical gate controlled diode characteristics $I_{\text {back }}$ as a function of $V_{\text {gate }}$ of the irradiated p-MOSFET <111>.

As presented in Fig. 7.9 (a) the surface current measurement has been performed for the p-MOSFET <111> irradiated with $E_{\text {field }} \downarrow$ after $16.61 \mathrm{kGy}$ X-ray dose for the three possible bias configurations. The same effects are observed as for the n-MOSFET measurement and this is evidence that the surface current measurement on MOSFETs is valid for both types of substrate p- and n-Si. In addition, in Fig. 7.9 (b) is presented the contribution of the interface traps to the surface current. The $\left|I_{\text {back }}\right|$ starts to increase due to $I_{\text {surf }}$ after $V_{f b}$ and the $\left|\phi_{s}\left(V_{\text {gate }}\right)\right|$ starts to increase from midgap to strong inversion as the $\left|V_{\text {gate }}\right|$ increases. The contribution of $I_{\text {surf }}$ to the $I_{\text {back }}$ and the increase of $\left|\phi_{s}\right|$ is presented approximately in the same gate voltage region $V_{\text {gate }}$, similar to the n-MOSFET measurements. 


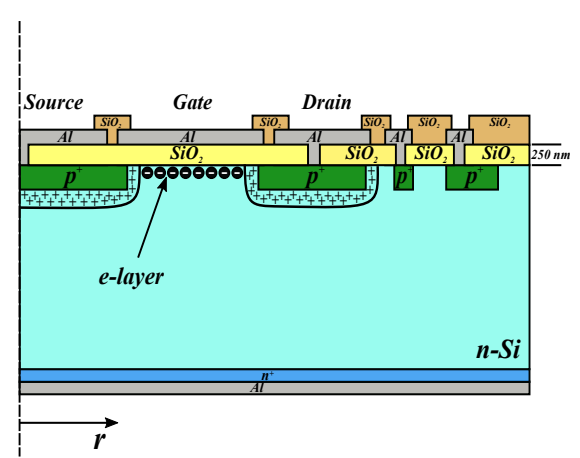

(a)

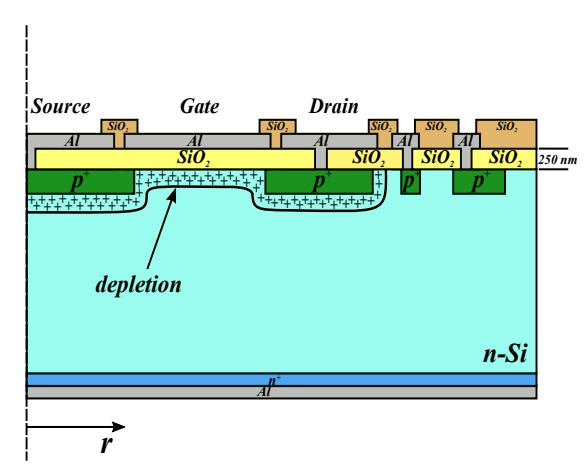

(c)

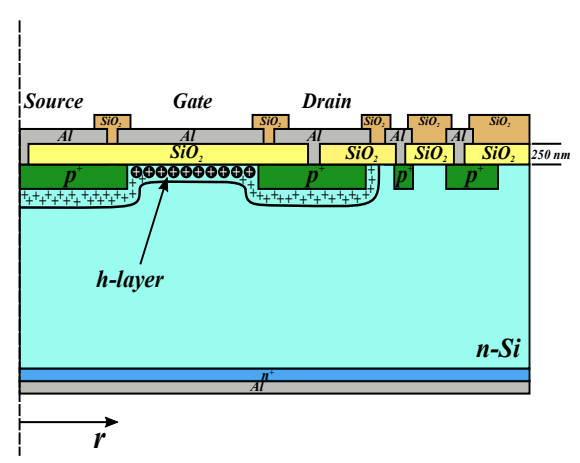

(e)

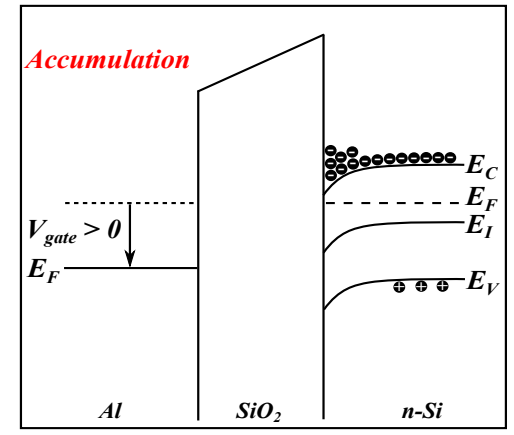

(b)

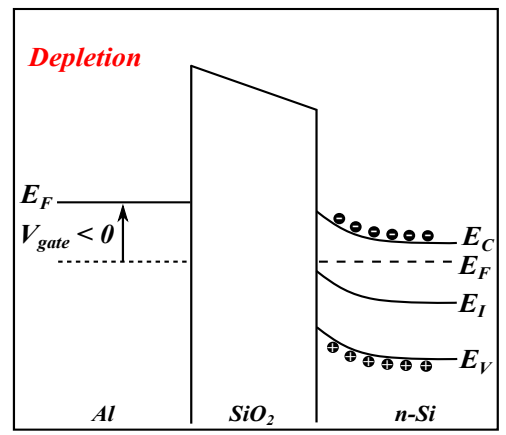

(d)

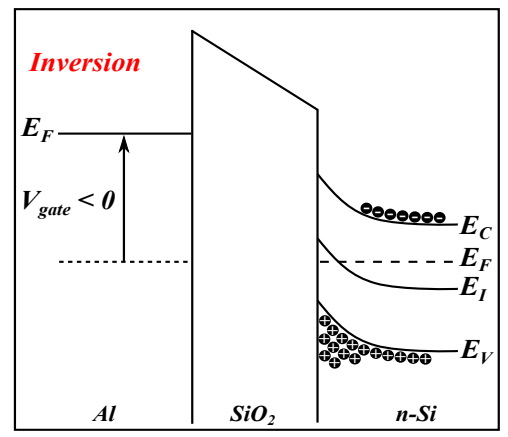

(f)

Figure 7.8: Cross section and Energy band diagram of the p-MOSFET $<111>$ in accumulation (a) and (b), depletion (c) and (d), inversion (e) and (f) respectively. 


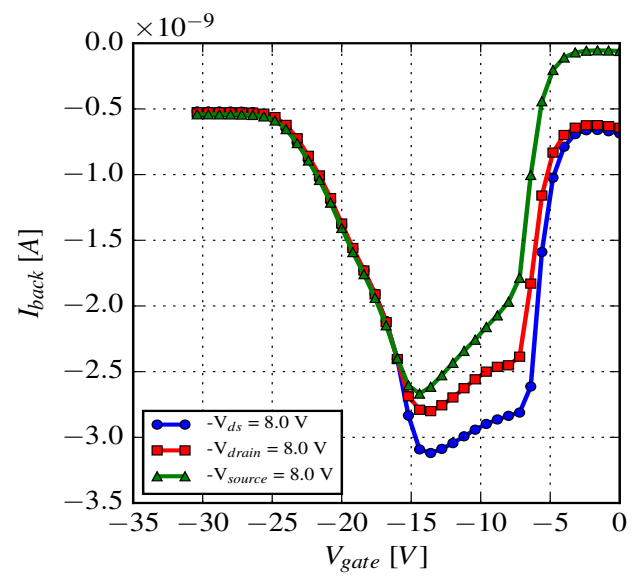

(a)

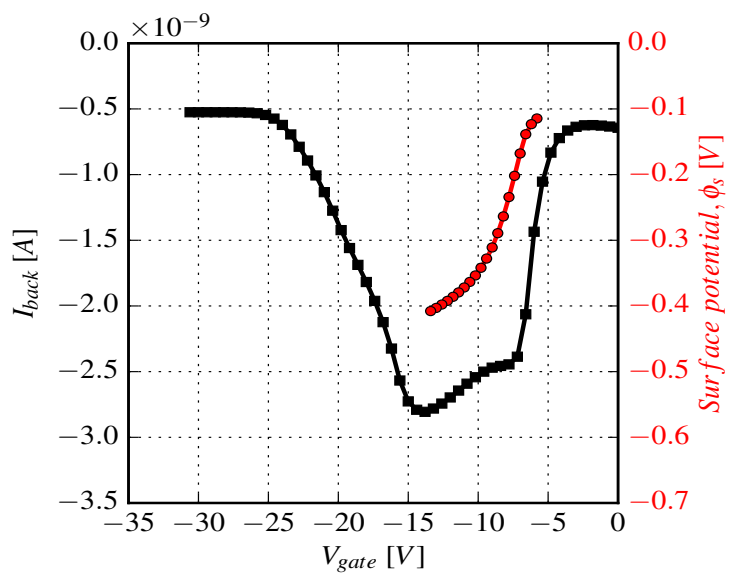

(b)

Figure 7.9: $I_{\text {back }}$ as a function of $V_{\text {gate }}$ of p-MOSFET <111> irradiated with $E_{\text {field }} \downarrow$ after $16.61 \mathrm{kGy}$ X-ray dose (a) for the three possible bias configurations and (b) with source floating, reverse voltage $-V_{d}=8 \mathrm{~V}$ on drain, and $\phi_{s}$ as a function of $V_{\text {gate }}$ extracted with the subthreshold current technique.

\subsection{Summary}

In this chapter is presented how a MOSFET can be used to measure the surface current. The method is similar to the measurements using a Gate Controlled Diode. In addition, is shown the correlation of the surface current with the surface potential. Results as a function of X-ray dose are presented for the nMOSFET irradiated with the electric field pointing into the $\mathrm{SiO}_{2}$. The values found are similar to the values obtained previously using a GCD irradiated without applied voltage. 


\section{Annealing study of oxide charge and interface traps}

Trapped positive charge annealing in MOS devices has been studied in detail in Refs. [85, 96,97, 102, 107, 129-133, 186-193]. Two main processes are proposed: (1) "true annealing" which occurs when the positive oxide charge is removed via trapped hole emission or electron tunneling and (2) "charge compensation" which occurs when electron trapping in the oxide leads to the formation of dipolar defects that wholly or partially neutralize the trapped positive charge. In this chapter is presented the annealing dependence of $N_{o x}^{e f f}, N_{o x}$ and $N_{i t}$ up to 256 hours at temperature of $60^{\circ} \mathrm{C}$.

\subsection{The p-MOSFET irradiated with $E_{\text {field }}$ pointing from the $\mathrm{Si}$ into the $\mathrm{SiO}_{2}$}

Annealing study of the p-MOSFET irradiated with $E_{\text {field }}$ pointing $\uparrow$ from the $\mathrm{Si}$ into the $\mathrm{SiO}_{2}$, has been performed. The test structure biased in inversion at a field in the $\mathrm{SiO}_{2}$ of about $500 \mathrm{kV} / \mathrm{cm}$ during irradiation and irradiated by X-rays up to a dose of about $17 \mathrm{kGy}\left(\mathrm{SiO}_{2}\right)$ in different irradiation steps. Before and after each irradiation, the gate voltage has been cycled from inversion to accumulation conditions and back and the threshold voltage and the hole mobility at the $\mathrm{Si}_{-} \mathrm{SiO}_{2}$ interface determined. The annealing was performed at a temperature of $60{ }^{\circ} \mathrm{C}$ without electric field across the gate. After each annealing step the sample was transferred $(\approx 10$ minutes delay time) from the oven to the measuring station and a measurement cycle has been applied. The cycle allows to study the annealing dependence of the effective oxide charge density, the charging-up and discharging of border traps and the hole mobility at the $\mathrm{Si}_{-} \mathrm{SiO}_{2}$ interface.

Measurement after annealing and analysis procedure Fig. 8.1 shows the measurement cycle adopted after each annealing step. On the top is shown, as a function of the measurement time, $t$, the applied gate voltage $V_{\text {gate }}(t)$, and on the bottom, the measured drain-source current $I_{d s}(t)$. $V_{\text {gate }}$ is changed from accumulation to inversion, $V_{i n v}$, kept at inversion for 3 hours, and then changed back to accumulation, $V_{a c c}$. After 2 minutes at $V_{a c c}$, this sequence is repeated. Two sequences are used, in order to check the reproducibility of the measurement results. The values of $V_{a c c}$ and $V_{i n v}$ for the annealing cycle are chosen 2.0 and $-38.0 \mathrm{~V}$ respectively, the same as for the last irradiation step of $10 \mathrm{kGy}$. The electric field in the oxide in accumulation, $E_{\text {field }}^{a c c}$, is varied from $\approx 830$ to $540 \mathrm{kV} / \mathrm{cm}$ and in inversion, $E_{\text {field }}^{i n v}$ from $\approx-830$ to $-1130 \mathrm{kV} / \mathrm{cm}$ for the different annealing steps.

The p-MOSFET has been annealed to a total time of 256 hours in steps of 1/6, 1/3, 1/2, 1, 2, 4, 6, 8, 16, $32,64,128$ hours. During the measurements and the storage, the p-MOSFET was kept at a temperature of about $23{ }^{\circ} \mathrm{C}$. From the measured $I_{d s}\left(V_{\text {gate }}\right)$ after annealing, for which $V_{\text {gate }}$ was changed from $V_{a c c}$ to $V_{i n v}$, 


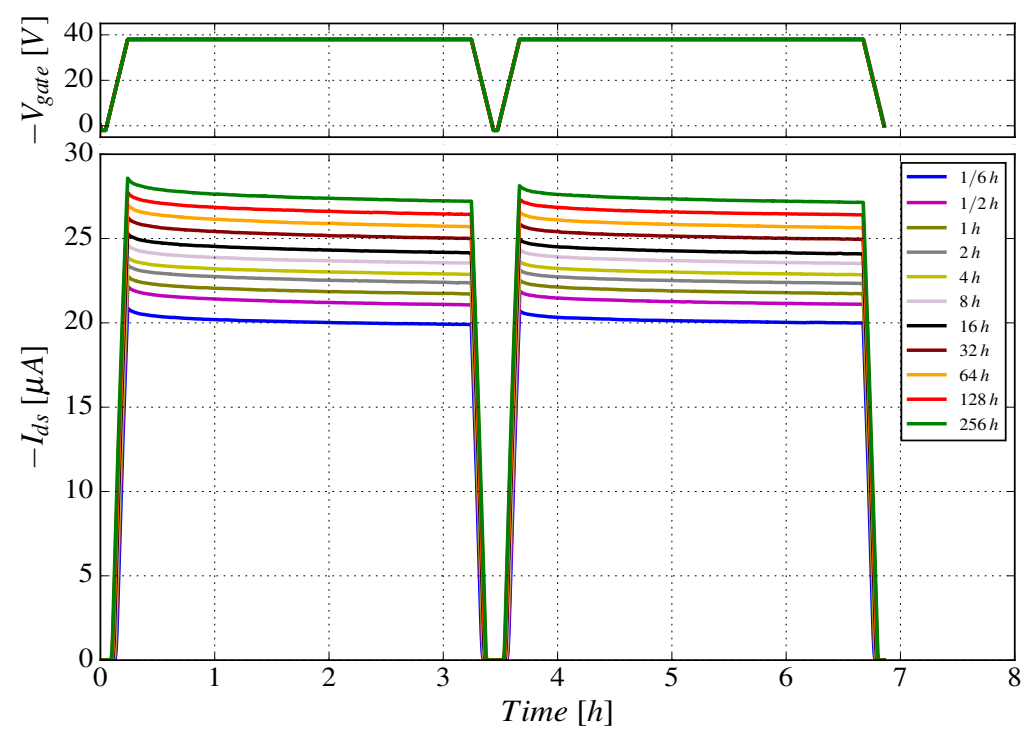

Figure 8.1: Measurement cycle after annealing: the applied gate voltage, $V_{\text {gate }}(t)$, is shown on the top, and the measured drain-source current, $I_{d s}(t)$, on the bottom of the p-MOSFET <111> irradiated with $E_{\text {field }} \uparrow$.

the values of $V_{t h}, \mu_{0}$ and $V_{1 / 2}$ are obtained using Eq. 5.1. From the $V_{t h}$ values the $N_{o x}^{e f f}$ is calculated using Eq. 5.5. Fig. 8.2 (a) shows the $I_{d s}\left(V_{\text {gate }}\right)$ calibration curves together with the fits for the different annealing steps.

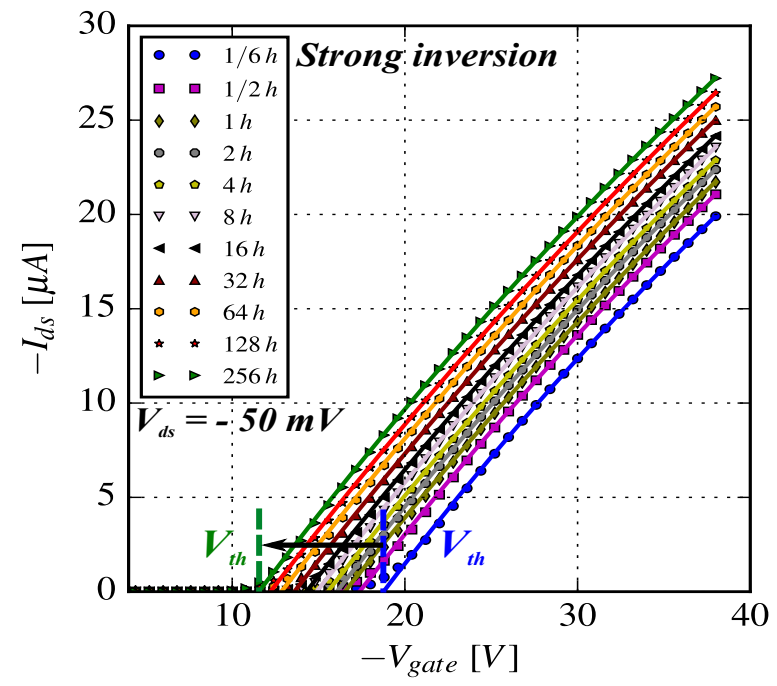

(a)

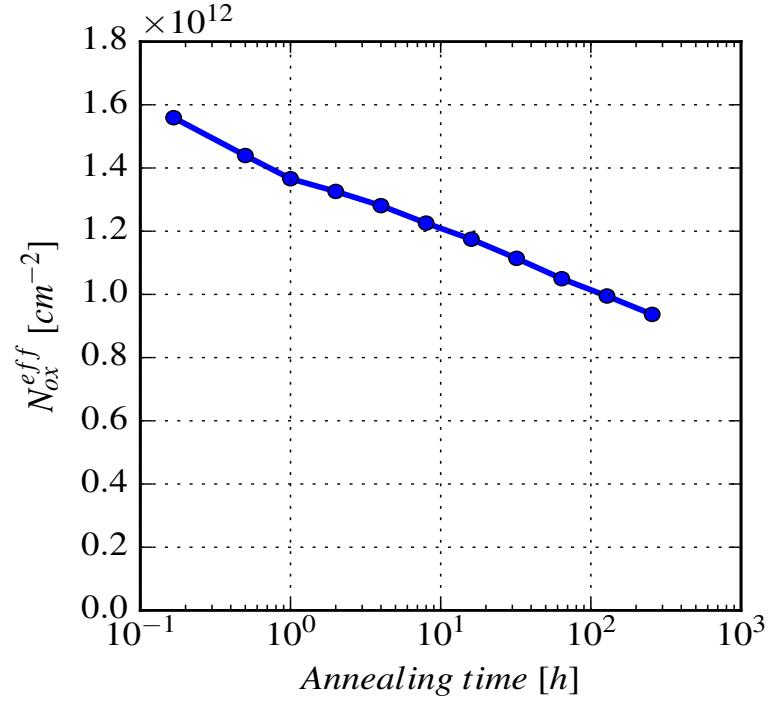

(b)

Figure 8.2: (a) $I_{d s}$ as a function of $V_{\text {gate }}$ in the linear region, and the results of the fit used to determine $V_{t h}$ and $\mu_{0}$ of p-MOSFET after annealing, and (b) Effective oxide charge density, $N_{o x}^{e f f}$ as a function of annealing time of the p-MOSFET $<111>$ irradiated with $E_{\text {field }} \uparrow$.

It is found that the values of $V_{t h}$ change from $\approx-18 \mathrm{~V}$ to $\approx-12 \mathrm{~V}$ due to the decrease of positive oxide charges. The slope of the $I_{d s}\left(V_{\text {gate }}\right)$ calibration curves is approximately constant with annealing time. In addition Fig. 8.1 shows that after every forward calibration, $I_{d s}$ decreases with time at the constant gate 
voltage $V_{\text {gate }}=V_{i n v}$. The time constant is of the order of 30 minutes. A decrease of $I_{d s}$ corresponds to an increase of $N_{o x}^{e f f}$. For $V_{g a t e}=V_{i n v}$ the p-MOSFET is in strong inversion: at the $\mathrm{Si}_{-} \mathrm{SiO}_{2}$ interface a hole inversion layer has formed, and the $E_{\text {field }}$ points $\uparrow$ from the $\mathrm{Si}$ into the $\mathrm{SiO}_{2}$. As a result, border traps are charged up positively. As seen in Fig. 8.1, the value of $I_{d s}$ at the start of the reverse calibration is lower than the value at the end of the forward calibration. This corresponds to a reduction of $N_{o x}^{e f f}$. For $V_{\text {gate }} \gg V_{t h}$, the p-MOSFET is in accumulation: at the $\mathrm{Si}_{-} \mathrm{SiO}_{2}$ interface an electron accumulation layer has formed, and the $E_{\text {field }}$ points $\downarrow$ from the $\mathrm{SiO}_{2}$ into the $\mathrm{Si}$. As a result, border traps are discharged.

Table 8.1: Conditions and extracted values of the surface radiation parameters as a function of annealing time.

\begin{tabular}{|c|c|c|c|c|c|c|c|c|c|c|}
\hline $\begin{array}{c}\text { Annealing time } \\
{[\mathrm{h}]}\end{array}$ & $\begin{array}{l}V_{a c c} \\
{[\mathrm{~V}]}\end{array}$ & $\begin{array}{l}V_{i n v} \\
{[\mathrm{~V}]} \\
\end{array}$ & $\begin{array}{c}E_{\text {field }}^{a c c} \\
{[\mathrm{kV} / \mathrm{cm}]}\end{array}$ & $\begin{array}{c}E_{\text {field }}^{\text {inv }} \\
{[\mathrm{kV} / \mathrm{cm}]}\end{array}$ & $\begin{array}{l}N_{\text {ox }, \text { ann }}^{\text {eff }} \\
{\left[\mathrm{cm}^{-2}\right]}\end{array}$ & $\begin{array}{l}\Delta N_{o x, c h} \\
{\left[\mathrm{~cm}^{-2}\right]}\end{array}$ & $\begin{array}{c}\Delta N_{\text {ox }, \text { disch }} \\
{\left[\mathrm{cm}^{-2}\right]}\end{array}$ & $\begin{array}{c}\mu_{0} \\
{\left[\mathrm{~cm}^{2} /(\mathrm{Vs})\right]}\end{array}$ & $\begin{array}{l}V_{1 / 2} \\
{[\mathrm{~V}]}\end{array}$ & $\begin{array}{c}\Delta \\
{[\mathrm{nm}]}\end{array}$ \\
\hline $1 / 6$ & 2.0 & -38.0 & 830 & -830 & $155.9 \cdot 10^{10}$ & $9.3 \cdot 10^{10}$ & $11.5 \cdot 10^{10}$ & 192 & -119 & 0.49 \\
\hline 1 & 2.0 & -38.0 & 740 & -930 & $136.6 \cdot 10^{10}$ & $10.4 \cdot 10^{10}$ & $10.1 \cdot 10^{10}$ & 184 & -153 & 0.38 \\
\hline 2 & 2.0 & -38.0 & 720 & -940 & $132.6 \cdot 10^{10}$ & $10.6 \cdot 10^{10}$ & $10.2 \cdot 10^{10}$ & 188 & -146 & 0.40 \\
\hline 4 & 2.0 & -38.0 & 700 & -960 & $128.1 \cdot 10^{10}$ & $10.1 \cdot 10^{10}$ & $10.4 \cdot 10^{10}$ & 187 & -149 & 0.39 \\
\hline 32 & 2.0 & -38.0 & 620 & -1050 & $111.4 \cdot 10^{10}$ & $12.3 \cdot 10^{10}$ & $10.3 \cdot 10^{10}$ & 190 & -153 & 0.38 \\
\hline 64 & 2.0 & -38.0 & 590 & -1080 & $105.0 \cdot 10^{10}$ & $13.0 \cdot 10^{10}$ & $9.2 \cdot 10^{10}$ & 190 & -156 & 0.37 \\
\hline 128 & 2.0 & -38.0 & 570 & -1110 & $99.5 \cdot 10^{10}$ & $12.8 \cdot 10^{10}$ & $11.3 \cdot 10^{10}$ & 191 & -156 & 0.37 \\
\hline 256 & 2.0 & -38.0 & 540 & -1130 & $93.7 \cdot 10^{10}$ & $13.5 \cdot 10^{10}$ & $10.2 \cdot 10^{10}$ & 193 & -154 & 0.38 \\
\hline
\end{tabular}

Table 8.1 presents for the individual annealing steps the integrated annealing time, the values of $V_{a c c}, V_{i n v}$ applied to the gate for the measurement cycle and the corresponding electric field values in accumulation, $E_{\text {field }}^{a c c}$, and inversion, $E_{\text {field }}^{\text {inv }}$. Fig. 8.2 (b) presents the values of $N_{o x}^{e f f}$ as a function of annealing time, $N_{o x}^{e f f}$ ann $(t)$ in the sixth column of Table 8.1. These values determined at the end of the first sequence of the cycle. Before the annealing, at an irradiation dose of $16.61 \mathrm{kGy}\left(\mathrm{SiO}_{2}\right), N_{o x, \text { ann }}^{\text {eff }}(0)=1.9 \cdot 10^{12} \mathrm{~cm}^{-2}$. For the maximum annealing time of 256 hours, $N_{o x, \text { ann }}^{e f f}(256 \mathrm{~h})=9.4 \cdot 10^{11} \mathrm{~cm}^{-2}$. From the values shown in the Table 8.1 as well as from Fig. 8.2 (b), one sees the $N_{o x, a n n}^{e f f}(t)$ has a decrease of $\approx 40 \%$ after 256 hours annealing.

Border traps and hole mobility Border traps, the near-interfacial oxide traps that communicate with the Si over a wide range of time scales, has been measured after each annealing step. The seventh column of Table 8.1 shows the total annealing time dependence of $\Delta N_{o x, c h}(t)$, the charging up of the border traps during the three hours after each annealing step, and the eighth column $\Delta N_{o x}$, disch $(t)$, the discharging of border traps, when changing $V_{\text {gate }}$ from inversion to accumulation, remaining 2 minutes in accumulation and then biasing back to inversion. A significant charging up and discharging of border traps, at the level of 5-15\% of $N_{o x}^{e f f}$, is observed. For the highest annealing time values, the $\Delta N_{o x, c h}(t)$ values start to increase and the $\Delta N_{o x, d i s c h}(t)$ stay approximately constant up to 256 hours of annealing at $60{ }^{\circ} \mathrm{C}$, shown in Fig. 8.3 (a). The electric field during charging, $\left|E_{\text {field }}^{i n v}\right|$, increases as a function of annealing time and during discharging, $E_{\text {field }}^{a c c}$, decreases due to $V_{t h}$ shift in the positive direction, as shown in Fig. 8.3 (b).

In addition, the $I_{d s}\left(V_{\text {gate }}\right)$ calibrations allow to determine the dependence of the hole mobility on $V_{\text {gate }}$ for different dose values. The parametrization is presented in Ch. 5. The results for $\mu_{0}$ and $V_{1 / 2}$ for the reverse calibration curves after the annealing steps are shown in Table 8.1 and in Fig. 8.4. Before the annealing, at the irradiation dose of $16.61 \mathrm{kGy}\left(\mathrm{SiO}_{2}\right)$ the extracted hole mobility at $E_{\text {field }} \approx 0$, is $\mu_{0}=200 \mathrm{~cm}^{2} /(\mathrm{V} \cdot \mathrm{s})$ and 


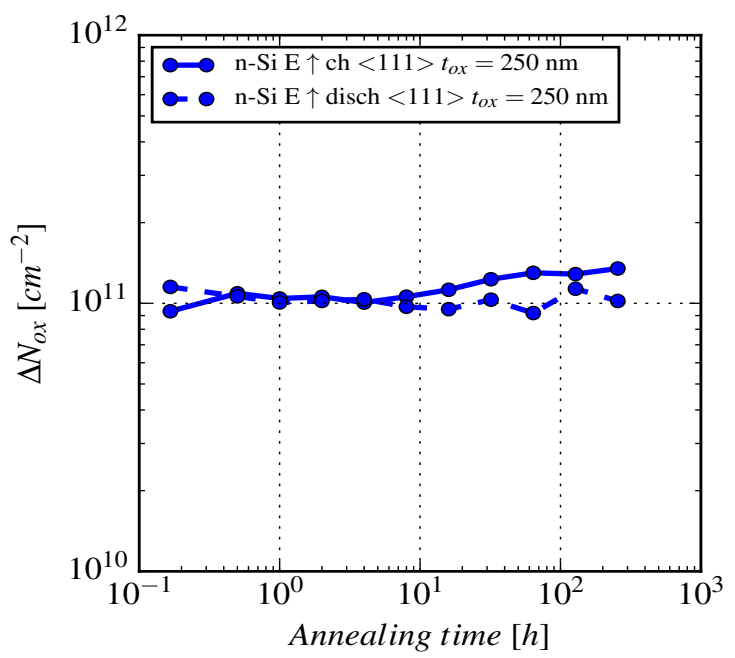

(a)

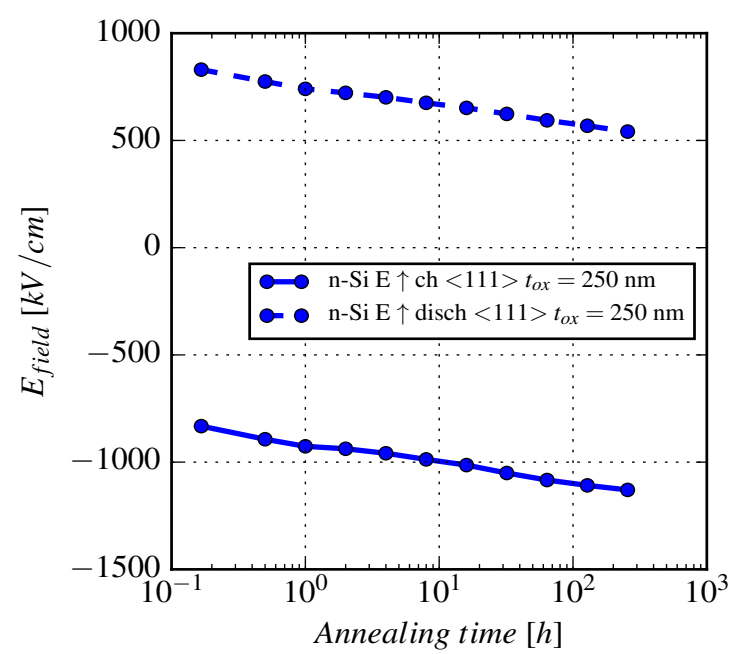

(b)

Figure 8.3: (a) $\Delta N_{o x}$ corresponding to the charging and discharging of border traps, and (b) $E_{\text {field }}$ values during the charging and discharging of border traps as a function of the annealing time of the p-MOSFET irradiated with $E_{\text {field }} \uparrow$.

after 256 hours annealing at $60{ }^{\circ} \mathrm{C}, \mu_{0}=193 \mathrm{~cm}^{2} /(\mathrm{V} \cdot \mathrm{s})$. As shown in Fig. 8.4 (a) $\mu_{0}$ stays approximately constant as a function of annealing time. The value of $V_{1 / 2}$ changes from $-119 \mathrm{~V}$ to about $-154 \mathrm{~V}$. In Fig. 8.4 (b) is presented the $V_{1 / 2}$ and the surface roughness amplitude $\Delta$ as a function of annealing time.

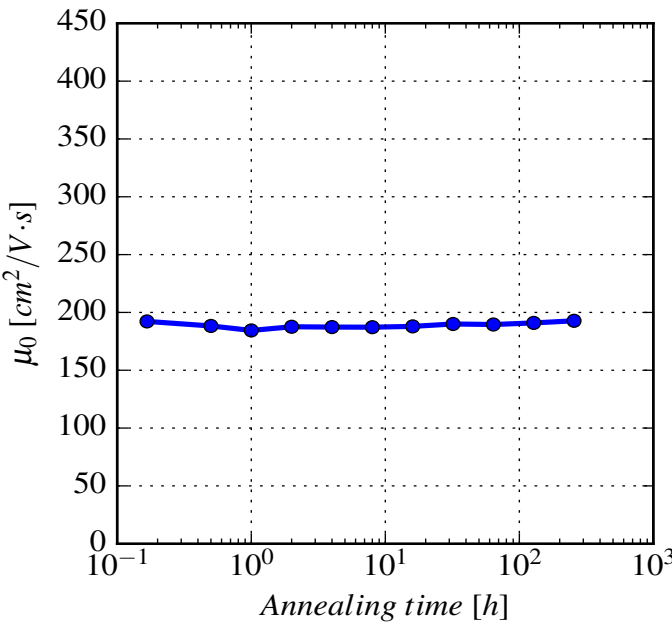

(a)

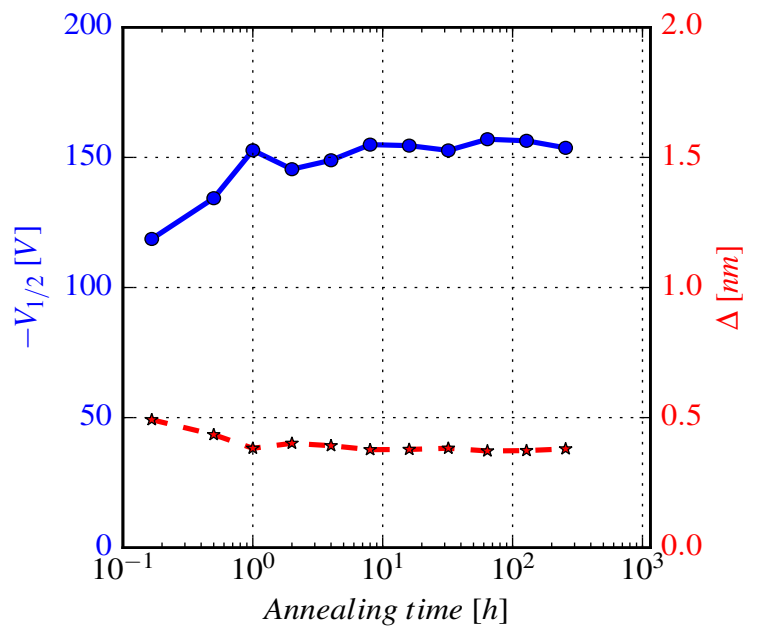

(b)

Figure 8.4: (a) Hole mobility, $\mu_{0}$, and (b) $V_{1 / 2}$ and $\Delta$ as a function of annealing time of the p-MOSFET $<111>$ irradiated with $E_{\text {field }} \uparrow$. 


\subsection{Annealing dependence of $N_{o x}, N_{i t}$ and $D_{i t}$}

The key to separating the contributions of oxide and interface trap charge using the subthreshold current technique is based on the assumption that radiation-induced interface traps are charge neutral at midgap [85, 104]. This assumption is generally consistent with electron-spin-resonance measurements [96], as well as capacitance-voltage [102,112] and conductance studies [168] performed on MOS capacitors. However doubts have been raised in Ref. [169] about the accuracy and the applicability of this assumption. The answer is presented below, as the subthreshold current technique provides a consistent description of the long-term annealing response of the irradiated p-MOSFET, which strongly supports the validity of the midgap charge neutrality. The annealing dependence of the fixed oxide charge density $N_{o x}$, the interface trap density $N_{i t}$ and the energy distribution of the interface traps $D_{i t}$ is extracted from the p-MOSFET $<111>$ irradiated with $E_{\text {field }}$ pointing $\uparrow$ from the $\mathrm{Si}$ into the $\mathrm{SiO}_{2}$ to a dose of $16.61 \mathrm{kGy}$. In Fig. 8.5 (a) is presented the $I_{d s}\left(V_{\text {gate }}\right)$ in log scale as a function of annealing time in the subthreshold region.

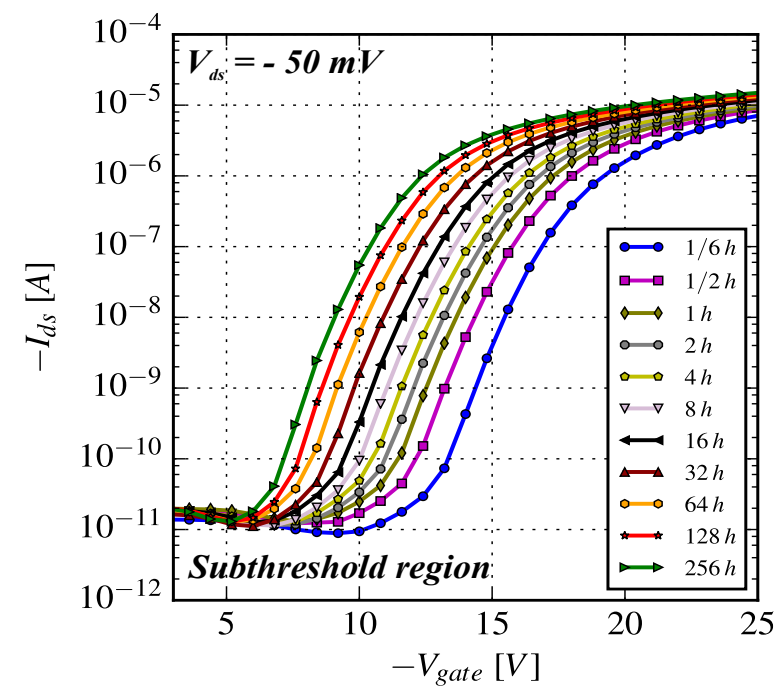

(a)

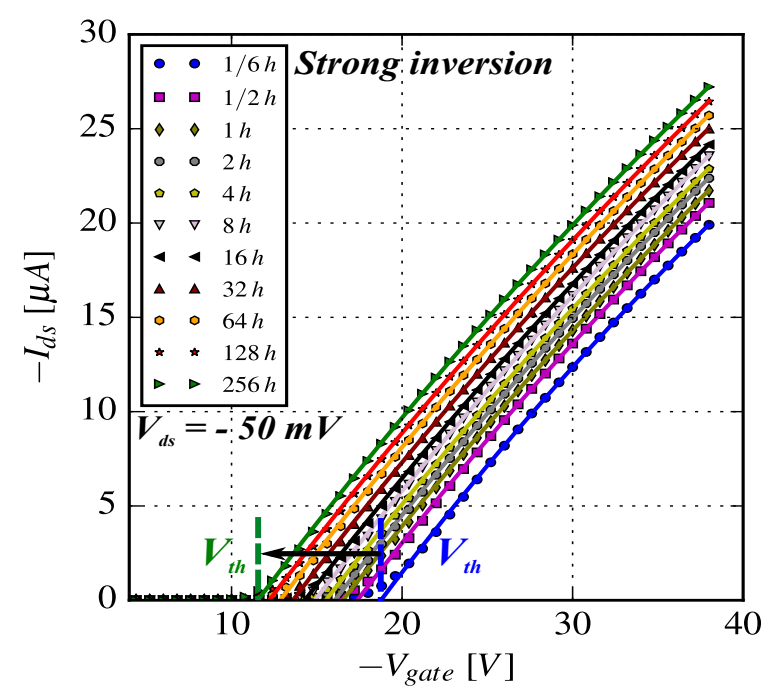

(b)

Figure 8.5: (a) $I_{d s}\left(V_{\text {gate }}\right)$ in the subthreshold region, (b) $I_{d s}\left(V_{\text {gate }}\right)$ in strong inversion and the parametrization model fit used to determine the threshold voltage, $V_{t h}$, as a function of the annealing time of the p-MOSFET $<111>$ irradiated with $E_{\text {field }} \uparrow$.

The subthreshold slope (stretchout) of the curves stays approximately constant and $V_{t h}$ shifts to the positive direction as the annealing time increases. Using the midgap method, the midgap voltage, $V_{m g}$, is determined after the calculation of the midgap current, $I_{m g}$, for each subthreshold curve. The calculated $I_{m g}$ and extracted $V_{m g}$ values are presented in Table 8.2. The fixed oxide charge density, $N_{o x}$, is calculated from the Eq. 6.18. The $V_{i t}$ "stretchout" is related to the presence of donor traps between midgap and threshold and calculated from Eq. 6.19, where the interface trap density, $N_{i t}$, is calculated from Eq. 6.20 with (-) for n-bulk Si.

The annealing dependence of $V_{t h}, V_{m g}$ and $V_{i t}$ is presented in Fig. 8.6 (a) and the calculated $N_{o x}^{e f f}, N_{o x}$ and $N_{i t}$ in Fig. 8.6 (b) for the p-MOSFET $<111>$ irradiated with $E_{\text {field }} \uparrow$. 
Table 8.2: Charge separation results as a function of annealing time of the p-MOSFET $<111>$ irradiated with $E_{\text {field }} \uparrow$.

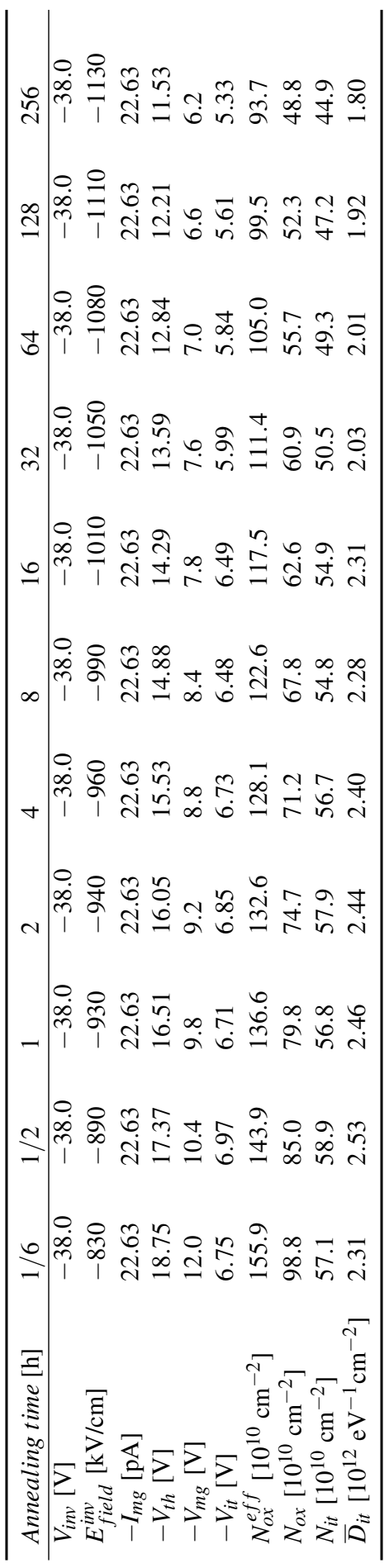




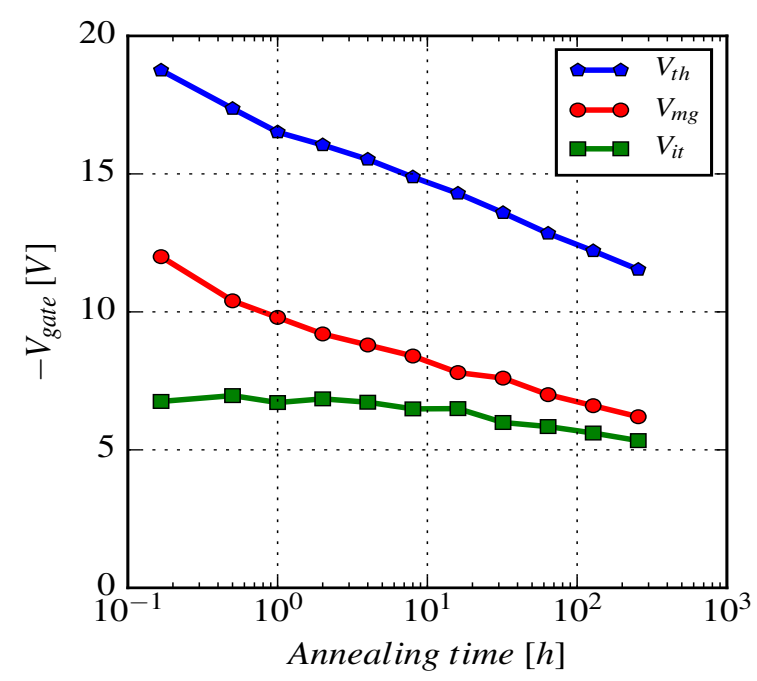

(a)

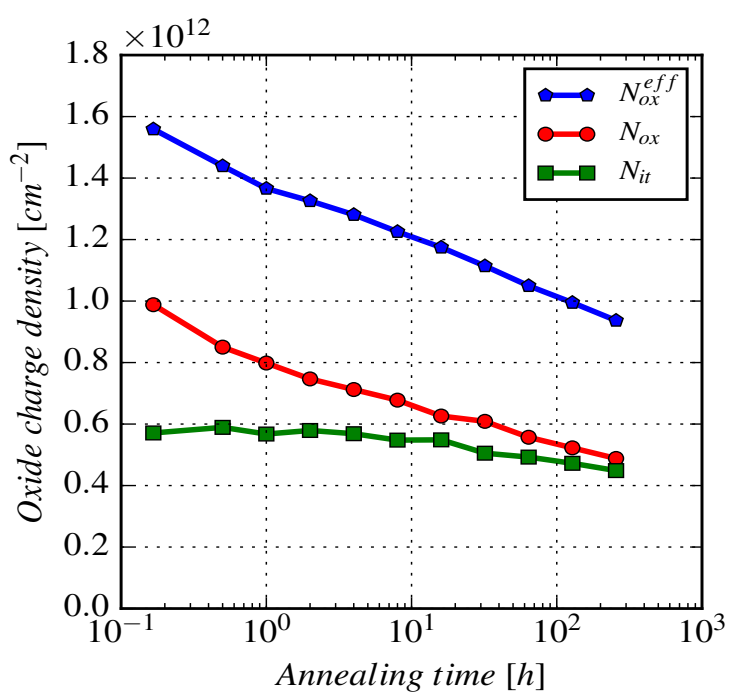

(b)

Figure 8.6: (a) Gate voltage and, (b) Oxide charge density as a function of annealing time of the p-MOSFET $<111>$ irradiated with $E_{\text {field }} \uparrow$.

$V_{\text {th }}$ shifts from $\approx-19.9 \mathrm{~V}$ after $16.61 \mathrm{kGy} \mathrm{X}$-ray dose, to $\approx-11.5 \mathrm{~V}$ after 256 hours annealing at $60{ }^{\circ} \mathrm{C}$. The contribution of $V_{m g}$ and $V_{i t}$ to the net shift is from $-12.0 \mathrm{~V}$ to $-6.2 \mathrm{~V}$ and from $\approx-7.9 \mathrm{~V}$ to $\approx-5.3 \mathrm{~V}$ respectively. The decrease in $V_{t h}$ is explained by the additive effect of positive charge decrease of interface traps and positive fixed oxide charge decrease to the total net $V_{t h}$. The $N_{o x}^{e f f}$ decreases from $\approx 1.7 \cdot 10^{12} \mathrm{~cm}^{-2}$ to $\approx 9.4 \cdot 10^{11} \mathrm{~cm}^{-2}, N_{o x}$ decreases from $\approx 9.9 \cdot 10^{11} \mathrm{~cm}^{-2}$ to $\approx 4.9 \cdot 10^{11} \mathrm{~cm}^{-2}$ and $N_{i t}$ decreases from $\approx 6.7 \cdot 10^{11} \mathrm{~cm}^{-2}$ to $\approx 4.5 \cdot 10^{11} \mathrm{~cm}^{-2}$.

In particular $N_{o x}$ decreases linearly with logarithmic time while $N_{i t}$ remains significant. The $N_{o x}$ neutralization can be accelerated by elevating the annealing temperature [106,137]. However high annealing temperatures remove all of the trapped holes also remove most or all of the interface traps [194] and no conclusion about the midgap charge neutrality can be extracted. A significant interface trap density still remains after annealing of 256 hours at $60{ }^{\circ} \mathrm{C}$ and the oxide charge can be extrapolated to its pre-irradiation value, as shown in Fig. 8.7. The small decrease of interface trap density as a function of logarithmic time, probably is related to the presence of "donor-like" border traps in the subthreshold region. Unfortunately the effective frequency $(\approx 1 \mathrm{~Hz}$ ) of the I-V measurement does not allow a further separation between border and interface traps. These results support the idea that the radiation-induced interface traps are charge neutral at midgap.

Similar to the analysis in Ch. 6, the $V_{\text {gate }}^{i t}\left(\phi_{s}\right)$ between midgap and threshold is determined after solving the non-linear equation $I_{d s}^{\text {ideal }}\left(\phi_{s}\right)-I_{d s}=0$. The solution gives the surface potential values, $\phi_{s}$, and the $V_{g a t e}^{i t}\left(\phi_{s}\right)$ as a function of annealing time and is presented in Fig. 8.8. The slope of the $V_{\text {gate }}^{i t}\left(\phi_{s}\right)$ curves shows a small decrease as a function of annealing due to the small decrease of the interface trap density. The density of interface traps as a function of surface potential, $D_{i t}\left(\phi_{s}\right)$, is calculated from Eq. 6.17 and presented in Fig. 8.9 (a). In addition, the energy distribution of interface traps, $D_{i t}\left(E_{i t}\right)$, where $E_{i t}=q_{0}\left(\phi_{s}-\phi_{b}\right)$ is calculated from the middle of the Si bandgap is presented in Fig. 8.9 (b). The $D_{i t}$ in the midgap position decreases from $\approx 7.0 \cdot 10^{12} \mathrm{eV}^{-1} \mathrm{~cm}^{-2}$ to $\approx 3.0 \cdot 10^{12} \mathrm{eV}^{-1} \mathrm{~cm}^{-2}$. At the energy of $0.28 \mathrm{eV}$ below midgap (threshold) the 


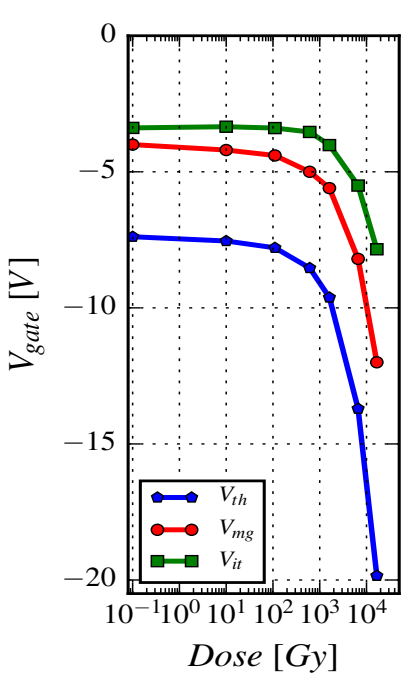

(a)

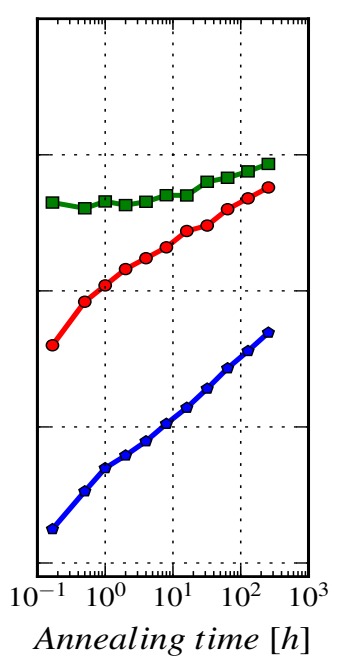

Annealing time $[h]$

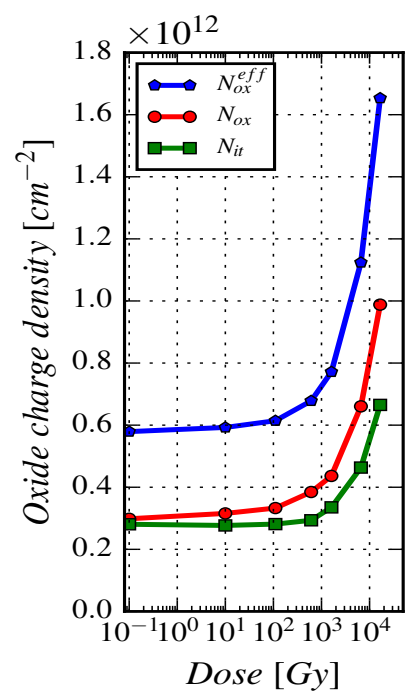

(b)

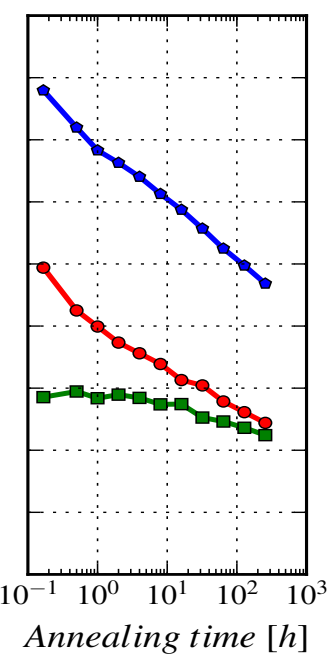

Figure 8.7: (a) Gate voltage and, (b) Oxide charge density as a function of X-ray dose and annealing time of the p-MOSFET $<111>$ irradiated with $E_{\text {field }} \uparrow$.

decrease is from $\approx 5.0 \cdot 10^{12} \mathrm{eV}^{-1} \mathrm{~cm}^{-2}$ to $\approx 2.6 \cdot 10^{12} \mathrm{eV}^{-1} \mathrm{~cm}^{-2}$ up to 256 hours annealing. The average value of $\bar{D}_{i t}$ in the energy range between midgap and threshold decreases from $\approx 2.8 \cdot 10^{12} \mathrm{eV}^{-1} \mathrm{~cm}^{-2}$ to $\approx 1.8 \cdot 10^{12} \mathrm{eV}^{-1} \mathrm{~cm}^{-2}$ and is higher than the integrated interface trap density, $N_{i t}$. The energy distribution of interface traps shows a minimum $0.1 \mathrm{eV}$ below midgap, $E_{I}$, and then increases towards the valence band edge, $E_{V}$. The half "U" shaped distribution of traps, in the lower part of the Si bandgap with a "peak" at midgap remains also after annealing of 256 hours at $60{ }^{\circ} \mathrm{C}$, as shown in Fig. 8.9.

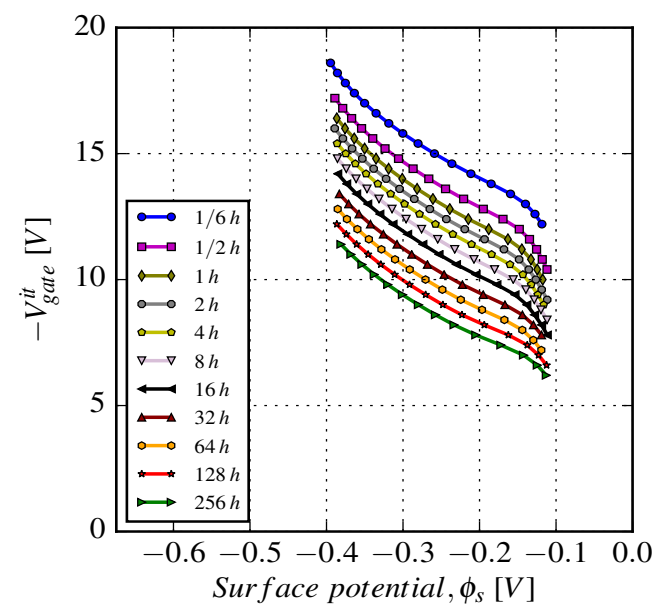

Figure 8.8: Gate voltage as a function of surface potential in the subthreshold region as a function of annealing time of the p-MOSFET $<111>$ irradiated with $E_{\text {field }} \uparrow$. 


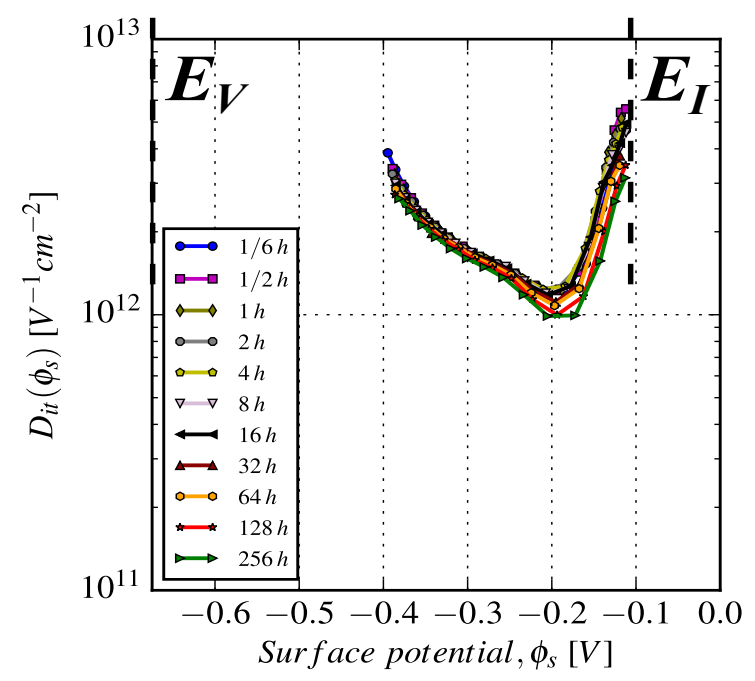

(a)

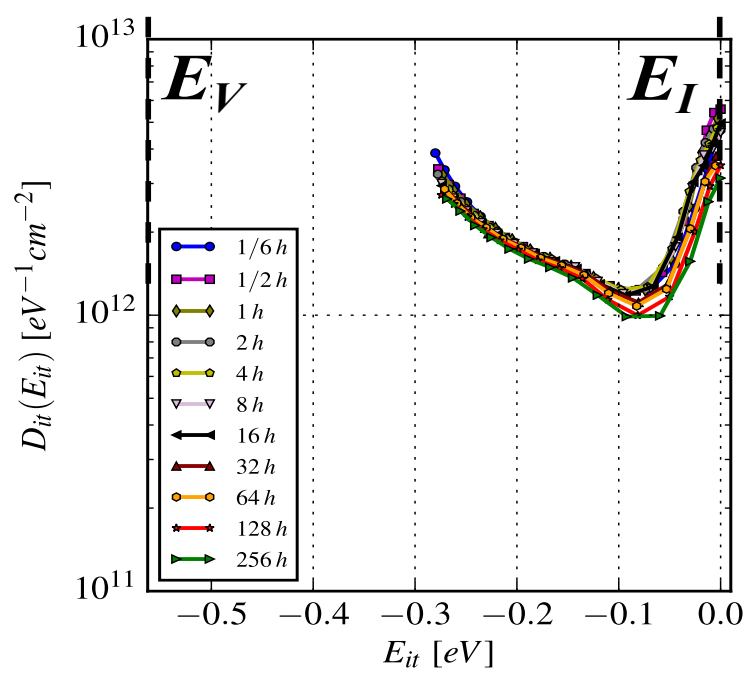

(b)

Figure 8.9: (a) $D_{i t}$ as a function of the surface potential and, (b) Energy in the Si bandgap as a function of annealing time of the p-MOSFET $<111>$ irradiated with $E_{\text {field }} \uparrow$.

\subsection{Summary}

In this chapter the annealing of oxide charges and interface traps for the p-MOSFET irradiated to a dose of about $17 \mathrm{kGy}$ with an electric field of approximately $500 \mathrm{kV} / \mathrm{cm}$ pointing from the $\mathrm{Si}$ into $\mathrm{SiO}_{2}$ has been studied. The annealing was made at $60{ }^{\circ} \mathrm{C}$ in different time steps up to 256 hours. It is found that the density of oxide charges decreases approximately linearly as a function of the logarithm of the annealing time, whereas the density of interface traps and the charging and discharging of border traps remains approximately constant. Also the mobility remains approximately constant, which confirms the observation that the interface traps do not anneal. The results are compatible with the assumption of the midgap charge neutrality, which is used for the extraction of the interface trap density from the subthreshold current. 



\section{Critical evaluation of the subthreshold current tech- nique}

In this chapter, the validity of the midgap method to determine the oxide charge and interface trap density is examined for different drain-source values, $V_{d s}$, in the linear region. In addition, are presented the influence of the gate voltage ramp rate, $V_{\text {ramp }}$, on the extracted parameters, the comparison between midgap and subthreshold swing method and the influence of the surface current on the drain-source current below strong inversion.

\subsection{Evaluation for different drain-source voltage and ramp rate}

Evaluation for different $V_{d s}$ values In order to apply the subthreshold charge separation technique and in particular the midgap method, the drain-source voltage $V_{d s}$ can be set for either linear or saturated operation in inversion [161]. If $V_{d s}$ is set for linear operation, the condition $V_{d s} \gg k T / q_{0}$ must be satisfied.

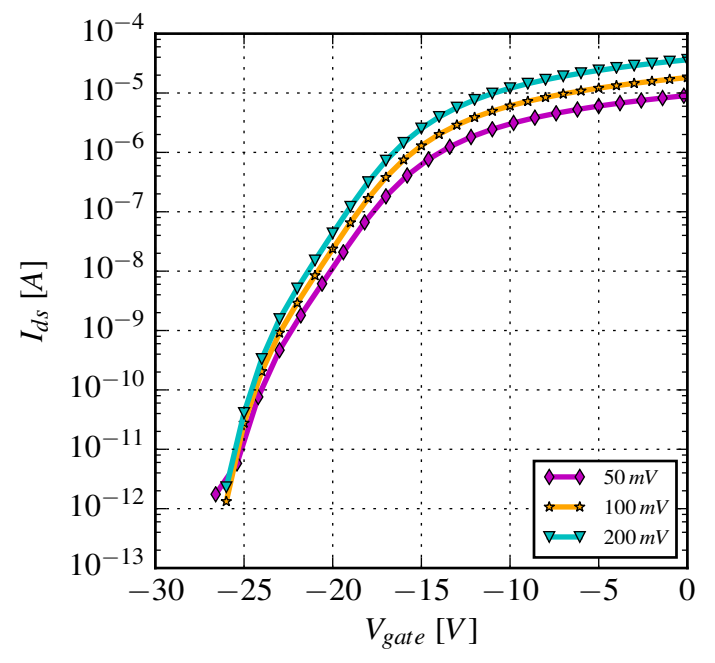

(a)

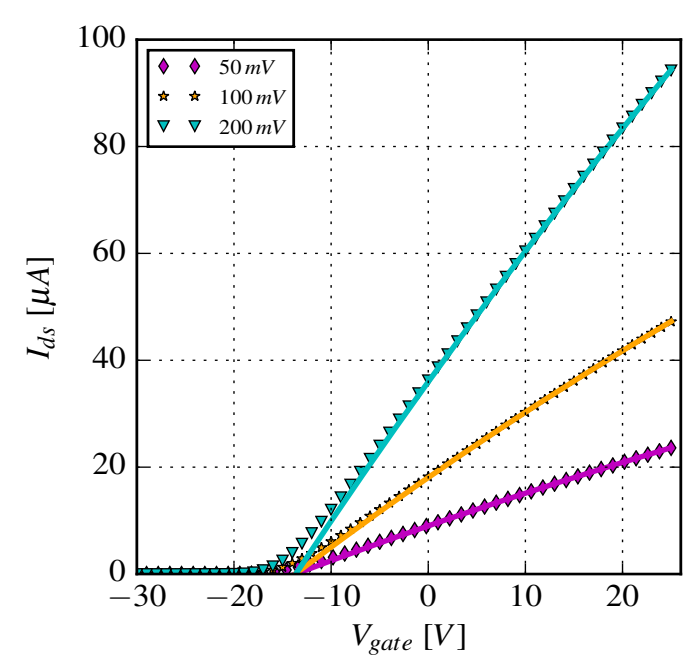

(b)

Figure 9.1: (a) $I_{d s}\left(V_{\text {gate }}\right)$ in the subthreshold region, and (b) $I_{d s}\left(V_{\text {gate }}\right)$ in strong inversion and the parametrization model fit used to determine the threshold voltage, $V_{t h}$, for different $V_{d s}$ values of the nMOSFET $<100>$ irradiated with $E_{\text {field }} \downarrow$ at $1.25 \mathrm{kGy}$ X-ray dose. 
Table 9.1: Charge separation results for different $V_{d s}$ values of the n-MOSFET $<100>$ irradiated with $E_{\text {field }}$ $\downarrow$ at $1.25 \mathrm{kGy} \mathrm{X}$-ray dose.

\begin{tabular}{llll}
\hline$V_{d s}[\mathrm{mV}]$ & 50 & 100 & 200 \\
\hline$V_{a c c}[\mathrm{~V}]$ & -55.0 & -55.0 & -55.0 \\
$V_{\text {inv }}[\mathrm{V}]$ & 25.0 & 25.0 & 25.0 \\
$E_{\text {field }}[\mathrm{kV} / \mathrm{cm}]$ & -590.3 & -590.0 & -590.9 \\
$\mu_{0}\left[\mathrm{~cm}^{2} /(\mathrm{Vs})\right]$ & 562.5 & 562.7 & 562.7 \\
$I_{m g}[\mathrm{pA}]$ & 4.66 & 4.66 & 4.66 \\
$V_{t h}[\mathrm{~V}]$ & -13.68 & -13.70 & -13.64 \\
$V_{m g}[\mathrm{~V}]$ & -25.6 & -25.8 & -26.0 \\
$V_{i t}[\mathrm{~V}]$ & 11.92 & 12.10 & 12.36 \\
$N_{o x}^{e f f}\left[10^{10} \mathrm{~cm}^{-2}\right]$ & 41.4 & 41.5 & 41.3 \\
$N_{o x}\left[10^{10} \mathrm{~cm}^{-2}\right]$ & 77.2 & 77.8 & 78.4 \\
$N_{i t}\left[10^{10} \mathrm{~cm}^{-2}\right]$ & -35.8 & -36.3 & -37.1 \\
$\bar{D}_{i t}\left[10^{12} \mathrm{eV}^{-1} \mathrm{~cm}^{-2}\right]$ & 1.31 & 1.29 & 1.26 \\
\hline
\end{tabular}

In the analysis of Chs. 6,8 , was chosen $V_{d s}=50 \mathrm{mV}$, where at room temperature $V_{d s} \simeq 2 k T / q_{0}$. The $V_{d s}=$ $50 \mathrm{mV}$ was selected in order to avoid short channel effects and large drain to substrate currents. So the validity of the method has to be checked for higher $V_{d s}$. The fixed oxide charge density, the interface trap density and the energy distribution of interface traps are extracted from the n-MOSFET $<100>$ irradiated with $E_{\text {field }}$ pointing $\downarrow$ from the $\mathrm{SiO}_{2}$ into the $\mathrm{Si}$ at $1.25 \mathrm{kGy}$ X-ray dose. In Fig. 9.1 (a) is presented the $I_{d s}\left(V_{\text {gate }}\right)$ in $\log$ scale for different $V_{d s}$ values in the subthreshold region.

The increase of the $I_{d s}$ in the subthreshold region for higher $V_{d s}$ values is related to the increase of the junction current between drain and substrate, which is measured as drain-source current at the source electrode. Using the midgap method, the midgap voltage, $V_{m g}$, is determined after the calculation of the midgap current, $I_{m g}$, for each subthreshold curve. The mobility $\mu_{0}$ values extracted in strong inversion, the data shown in Fig. 9.1

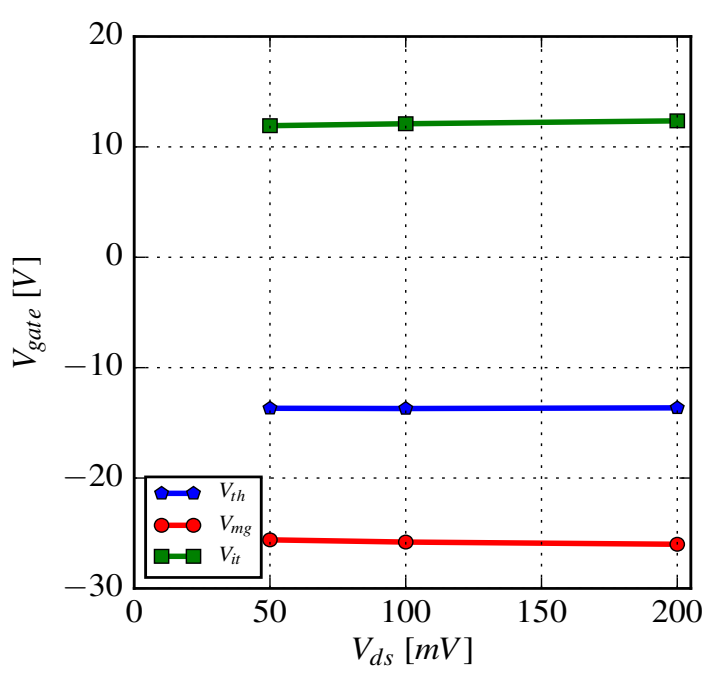

(a)

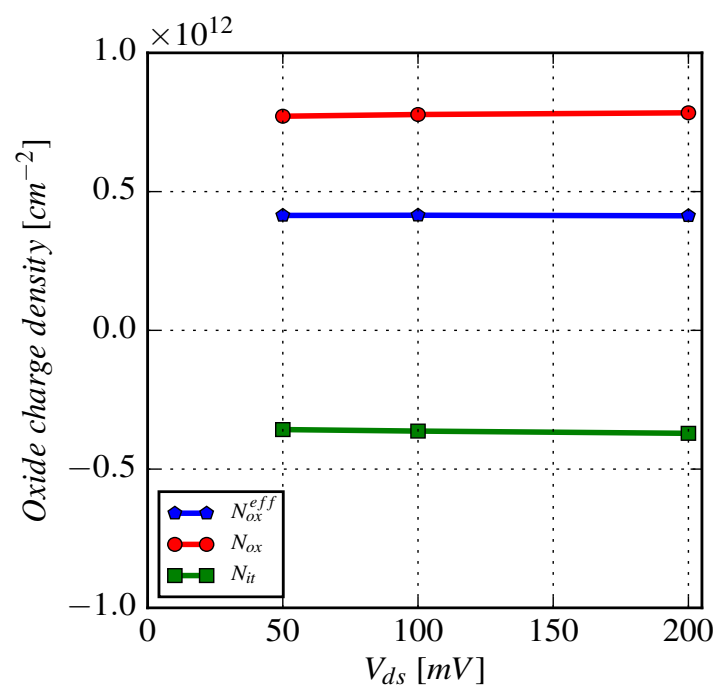

(b)

Figure 9.2: (a) Gate voltage and, (b) Oxide charge density for different $V_{d s}$ values of the n-MOSFET $<100>$ irradiated with $E_{\text {field }} \downarrow$ at $1.25 \mathrm{kGy}$ X-ray dose. 


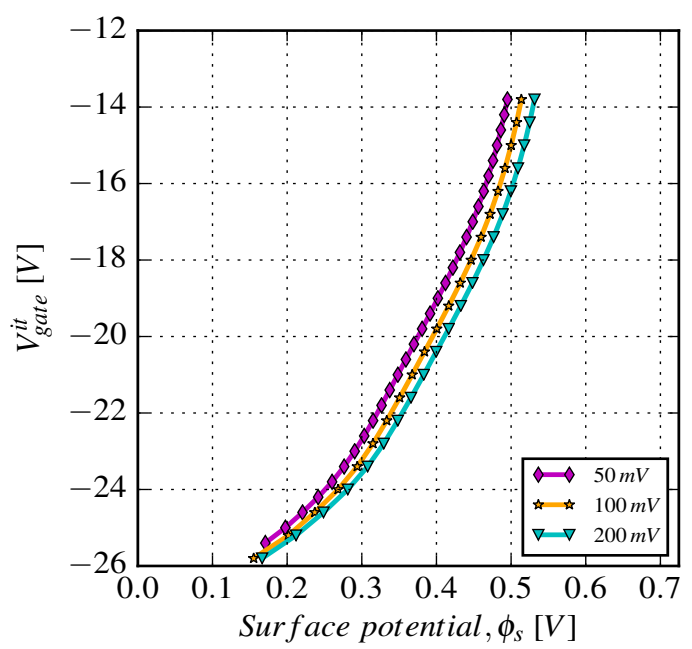

Figure 9.3: Gate voltage as a function of surface potential in the subthreshold region for different $V_{d s}$ values of the n-MOSFET $<100>$ irradiated with $E_{\text {field }} \downarrow$ at $1.25 \mathrm{kGy}$ X-ray dose.

(b), are used for the calculation of $I_{m g}$. As presented in Table 9.1, $\mu_{0}$ is found to be independent of $V_{d s}$, so the calculated $I_{m g}$ is constant and the extracted $V_{m g}$ values show a difference of $0.2 \mathrm{~V}$.

The fixed oxide charge density, $N_{o x}$, is calculated from Eq. 6.18. The $V_{i t}$ "stretchout" is related to the presence of acceptor traps between midgap and threshold and calculated from Eq. 6.19, where the interface trap density, $N_{i t}$, is calculated from Eq. 6.20 with (+) for p-bulk Si. The $V_{d s}$ dependence of $V_{t h}, V_{m g}$ and $V_{i t}$ is presented in Fig. 9.2 (a) and the calculated $N_{o x}^{e f f}, N_{o x}$ and $N_{i t}$ in Fig. 9.2 (b) for the n-MOSFET $<100>$ irradiated with $E_{\text {field }} \downarrow$ at $1.25 \mathrm{kGy}$ X-ray dose. In particular, no $V_{d s}$ dependence is observed for the extracted parameters. Similar as presented in previous chapters for n-MOSFET, the contribution of interface traps and fixed oxide charge is explained by the subtractive effect to the net $V_{t h}$. In order to determine the $V_{d s}$

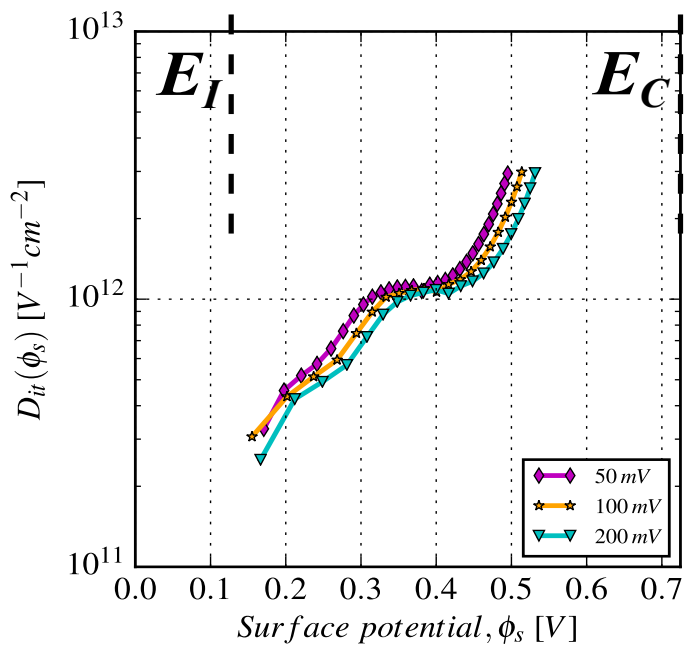

(a)

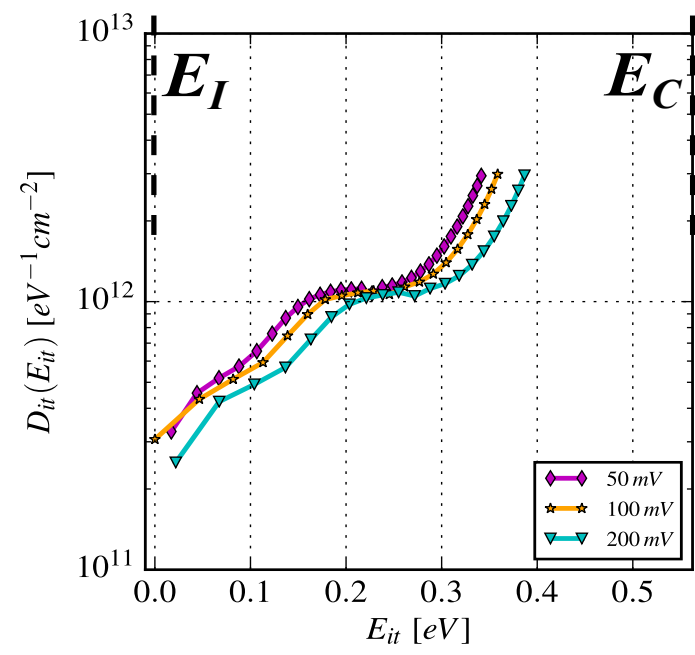

(b)

Figure 9.4: (a) $D_{i t}$ as a function of the surface potential and, (b) Energy in the Si bandgap for different $V_{d s}$ values of the n-MOSFET $<100>$ irradiated with $E_{\text {field }} \downarrow$ at $1.25 \mathrm{kGy}$ X-ray dose. 
dependence on the $D_{i t}$, the $V_{\text {gate }}^{i t}\left(\phi_{s}\right)$ between midgap and threshold is determined after solving the non-linear equation $I_{d s}^{\text {ideal }}\left(\phi_{s}\right)-I_{d s}=0$. The solution gives the surface potential values, $\phi_{s}$, and the $V_{\text {gate }}^{\text {it }}\left(\phi_{s}\right)$ for different $V_{d s}$ values presented in Fig. 9.3. The shift of the $V_{g a t e}^{i t}\left(\phi_{s}\right)$ to higher potential values with the increase of $V_{d s}$, is related on the increase of the quasi-Fermi level of the channel electrons. The density of interface traps as a function of surface potential, $D_{i t}\left(\phi_{s}\right)$, is calculated from Eq. 6.17 and presented in Fig. 9.4 (a). In addition the energy distribution of interface traps, $D_{i t}\left(E_{i t}\right)$, where $E_{i t}=q_{0}\left(\phi_{s}-\phi_{b}\right)$ is calculated from the middle of the Si bandgap is presented in Fig. 9.4 (b). The shift is also presented in the calculation of $D_{i t}\left(\phi_{S}\right)$ or $D_{i t}\left(E_{i t}\right)$ due to the effect on $V_{\text {gate }}^{\text {it }}\left(\phi_{s}\right)$.

Evaluation for different voltage ramp rates According to Refs. [102, 129] the calculation of interface trap density from MOS capacitors (Capacitance-Voltage measurements) shows a gate voltage ramp rate, $V_{\text {ramp }}$, dependence. It is observed that the "stretchout" of these $\mathrm{C}-\mathrm{V}$ curves from midgap to inversion strongly depends on the ramp rate used to measure the curve. The "stretchout" increases with the reduction of the $V_{\text {ramp }}$, due to the larger number of interface states that respond between midgap and inversion. In order to check the validity of the midgap method and the influence of the results presented in previous chapters on $V_{\text {ramp }}$, three ramp rate values, $0.87,0.93$ and $1.01 \mathrm{~V} / \mathrm{s}$, were tested on the n-MOSFET $<100>$ irradiated with $E_{\text {field }} \downarrow$ to $1.25 \mathrm{kGy}$ X-ray dose. The $V_{d s}$ dependence of $V_{t h}, V_{m g}$ and $V_{i t}$ is presented in Fig. 9.5 (a) and the calculated $N_{o x}^{e f f}, N_{o x}$ and $N_{i t}$ in Fig. 9.5 (b) for $V_{d s}=50 \mathrm{mV}$.

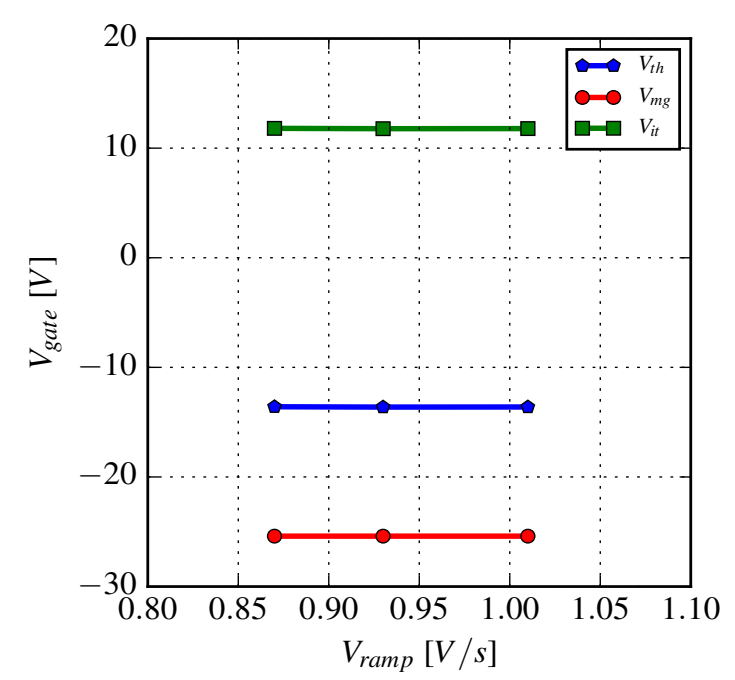

(a)

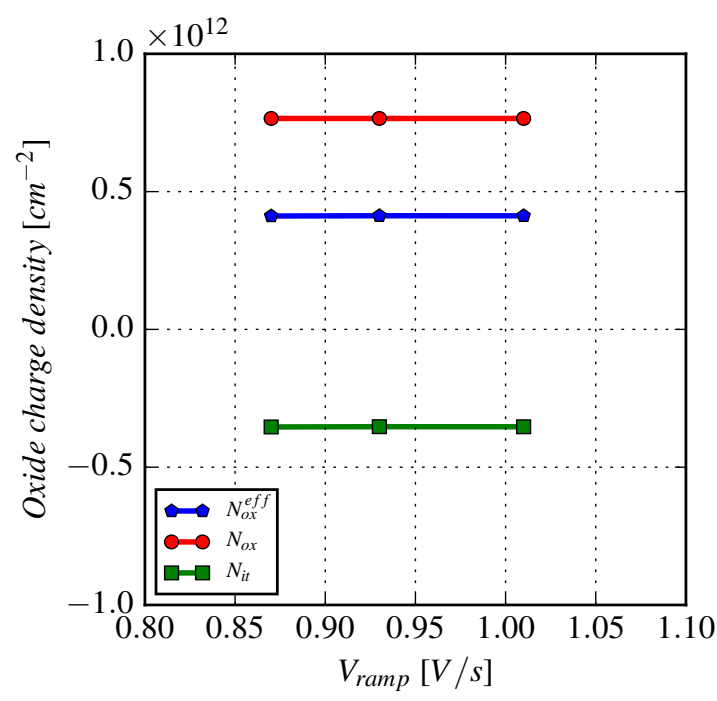

(b)

Figure 9.5: (a) Gate voltage and, (b) Oxide charge density for different $V_{\text {ramp }}$ values of the n-MOSFET $<100>$ irradiated with $E_{\text {field }} \downarrow$ at $1.25 \mathrm{kGy}$ X-ray dose.

No $V_{\text {ramp }}$ dependence is observed for the selected ramp rates in the slow regime. For the calculation of $D_{i t}$, the $V_{\text {gate }}^{\text {it }}\left(\phi_{s}\right)$ between midgap and threshold is determined for different $V_{\text {ramp }}$ values and presented in Fig. 9.6 (a). The curves are identical, which verifies that there is no $V_{\text {ramp }}$ dependence of the subthreshold "stretchout" in the slow ramp regime. Also, the energy distribution of the interface traps, $D_{i t}\left(E_{i t}\right)$, for the three different $V_{\text {ramp }}$ values are identical, as shown in Fig. 9.6 (b). 


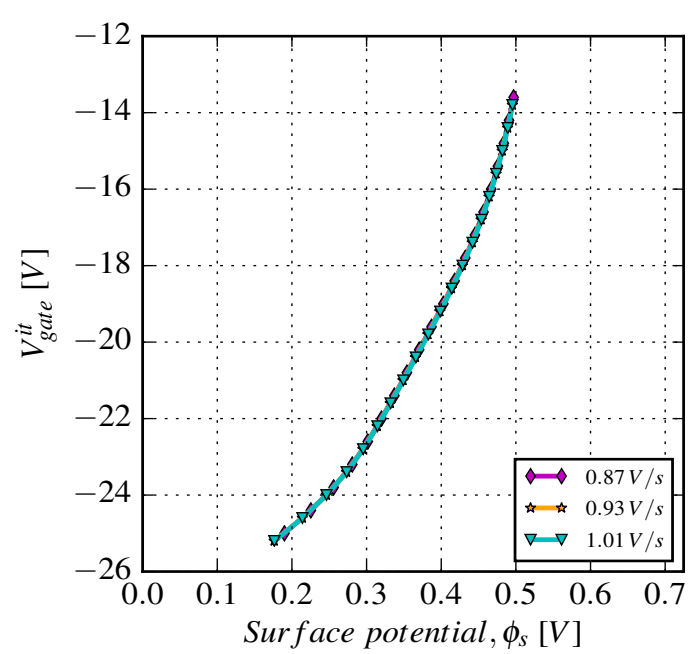

(a)

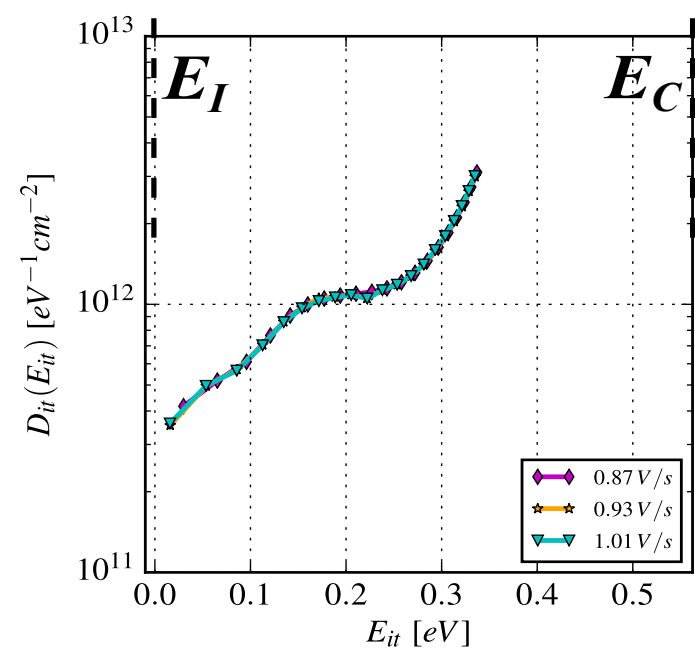

(b)

Figure 9.6: (a) Gate voltage as a function of surface potential in the subthreshold region and, (b) $D_{i t}$ as a function of energy in the Si bandgap for different $V_{\text {ramp }}$ values of the n-MOSFET $<100>$ irradiated with $E_{\text {field }} \downarrow$ at $1.25 \mathrm{kGy}$ X-ray dose.

\subsection{Evaluation of midgap and subthreshold swing method}

\subsubsection{The effect of charge lateral nonuniformities}

In this paragraph the two methods of the subthreshold charge separation technique are compared on the post-irradiation response of the n-MOSFET $<100>$ irradiated with $E_{\text {field }} \downarrow$. As described above, the primary method used to analyze the MOSFETs was the midgap method, similar to the high frequency Terman analysis $[170,187]$. The main idea of the method is the mapping of the surface potential to the gate voltage $V_{\text {gate }}$, by calculating the subthreshold current as a function of the surface potential, $I_{d s}^{\text {ideal }}\left(\phi_{s}\right)$. By solving the non-linear equation $I_{d s}^{\text {ideal }}\left(\phi_{s}\right)$ minus the measured $I_{d s}$, the calculated subthreshold current is related to the measured $I_{d s}\left(V_{\text {gate }}\right)$, for the appropriate surface potential $\phi_{s}$. Recording the $V_{\text {gate }}$ which resulted in the measured $I_{d s}$, the $V_{\text {gate }}\left(\phi_{s}\right)$ is mapped in this way. Then, using Eq. 6.17 the $D_{i t}\left(\phi_{s}\right)$ is calculated over a limited region of the Si bandgap. The analysis assumes that the interface traps are acceptors in the upper part of the bandgap and donors in the lower $[96,161,187]$. While this assumption remains an area of argumentation $[168,169,187]$, the increase in the "stretchout" of the subthreshold curves indicates the increase in the number of interface traps between midgap and threshold, independent of the validity of the assumption. However, also charge lateral nonuniformities LNUs can cause a "stretchout" similar to that caused by interface traps $[135,161,195-197]$. As a result, the voltage shift due to interface traps is overestimated. LNUs are differences in the oxide charge density at different locations on the $\mathrm{Si}_{-} \mathrm{SiO}_{2}$ interface along the channel. The surface potential is directly affected by the LNUs. Irregularities of the surface potential across the MOSFET channel can cause preferred current paths, so that at given surface potential the channel is not conducting uniformly. The effect of LNUs on a MOSFET channel can be simulated as many parallel transistors all with different threshold voltages but identical subthreshold curves, as presented in Fig. 9.7. 
In Figs. 9.7 (a) and 9.7 (c) the different regions at the $\mathrm{Si}_{-} \mathrm{SiO}_{2}$ interface are split into a group of parallel transistors, each associated with a different amount of oxide charge. Fig. 9.7 (a) presents curves where the differences in the oxide charge are large and Fig. 9.7 (c) presents curves where the oxide charge is more homogeneous. Fig. 9.7 (b) presents the composite subthreshold curves resulting from these two groups of parallel transistors. The regions with high oxide charge density will "turn on" at a lower gate voltage than those regions with lower oxide charge densities. The net effect is a "stretchout" of the subthreshold curves. The case with the larger LNU results in a reduction of the composite subthreshold slope and an increase of the subthreshold swing [135].

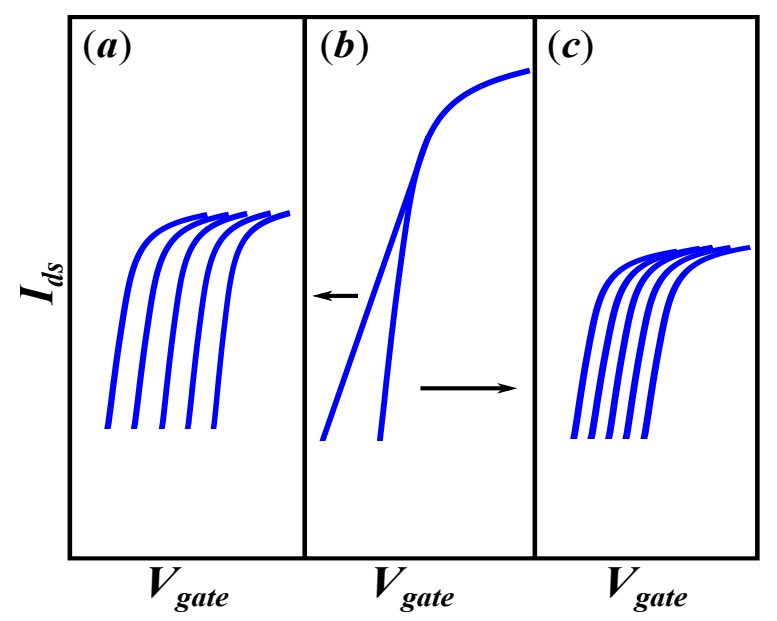

Figure 9.7: Schematic representation of the effect of lateral nonuniformities LNUs in trapped charge on the shape of the subthreshold curve. (a) Hypothetical set of curves of parallel transistors with significant LNUs, (c) Comparable case where LNUs are less serious, and (b) Resulting composite curves obtained from the parallel transistors (a) and (c). Figure adapted from [135].

The creation of LNUs are radiation bias, dose rate, and energy dependent. Low energy high dose rate radiation such from an X-ray source seem to produce greater LNU effects than $\mathrm{Co}^{60}$ radiation [135,161]. The effects of LNUs appear to anneal at room temperature in relatively short times, less than an hour [135, 161], however are more stable at cryogenic temperatures [195]. LNUs in irradiated MOSFETs can be identified by annealing of the interface traps (reduction of subthreshold swing) at room temperature at early times after irradiation. For this reason in Ch. 6, the subthreshold current technique and in particular the midgap method has been applied on the last part of the post-irradiation cycle, in the subthreshold curves after a few hours biasing in both states ("on" and "off"). In addition, the increase of interface traps after irradiation is compatible with the decrease of the mobility of holes and electrons at the $\mathrm{Si}_{-} \mathrm{SiO}_{2}$ interface, as observed in Ch. 5 .

\subsubsection{Comparison between midgap and subthreshold swing method}

As a further check of the midgap method on the extracted density of interface traps, $D_{i t}$, the subthreshold swing is calculated for curves of the n-MOSFET $<100>$ irradiated with $E_{\text {field }} \downarrow$. As described in Refs. [28, $165,198]$ and in Ch. 6 , the swing $\mathrm{S}$ is defined as the gate voltage needed to reduce the current by one decade, as the current in the subthreshold region varies as $\sim \exp \left(q_{0} \phi_{s} / \mathrm{kT}\right)$. As shown in Fig. 9.8 (a), the subthreshold 
swing $S$ as a function of $V_{\text {gate }}$ for different X-ray doses is calculated for the n-MOSFET irradiated up to $6.61 \mathrm{kGy} \mathrm{X}$-ray dose. The swing $S$, increases with X-ray dose due to the increase of interface traps between midgap and threshold. The change in the density of interface traps $\Delta D_{i t}$, as a function of the surface potential $\phi_{s}[102,161,198]$ is calculated from

$$
\Delta D_{i t}\left(\phi_{s}\right)=\frac{\Delta S\left(\phi_{s}\right) C_{o x}}{k T \ln 10}
$$

where $\Delta S\left(\phi_{s}\right)$ is the difference between the pre- and post-irradiation subthreshold swings. In Fig. 9.8 (b) is presented the $\Delta D_{i t}$ as a function of the surface potential for different X-ray doses, calculated from the midgap method (points) and the subthreshold swing method (line). The agreement between the two methods is satisfactory. The swing $\mathrm{S}$ has been calculated in the same subthreshold region between midgap and threshold, as used for the midgap method. The two methods have been applied on subthreshold measurements in the forward direction of the last part of the post-irradiation cycle.

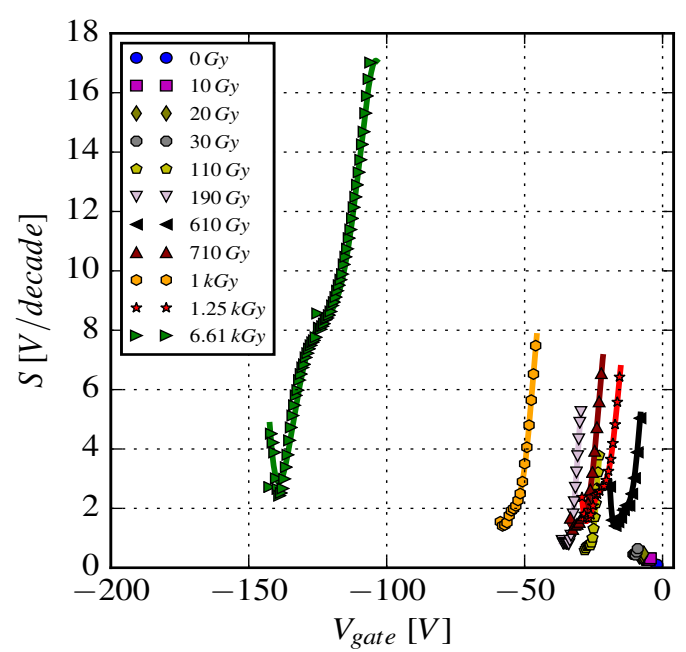

(a)

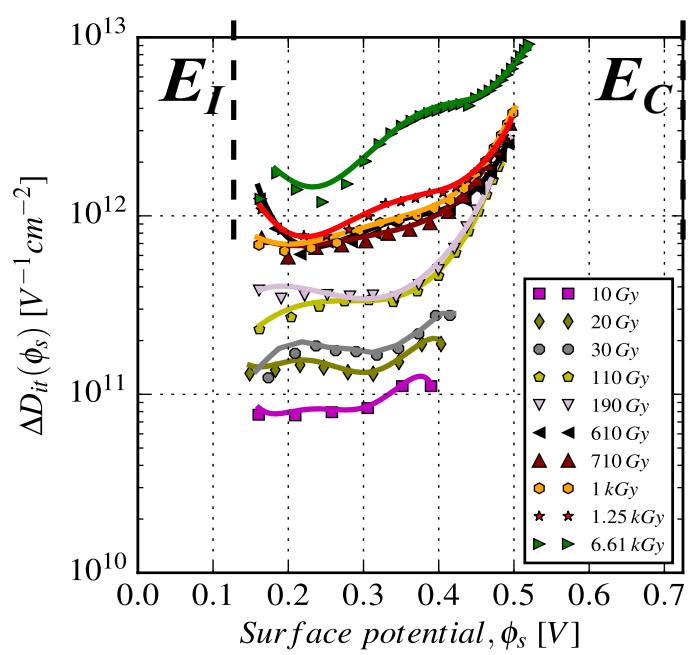

(b)

Figure 9.8: (a) Subthreshold swing $S$ as a function of $V_{\text {gate }}$ data points and interpolation straight line of the data and, (b) $\Delta D_{i t}$ as a function of the surface potential, calculated from the midgap method (points) and the subthreshold swing method (line) of the n-MOSFET <100> irradiated with $E_{\text {field }} \downarrow$.

\subsection{Surface current contribution on the subthreshold curve}

As indicated in Ch. 6 the validity of the subthreshold current technique depends on the diffusion current which flows in the channel in the weak inversion. This current should not influenced from gate and substrate leakages, other parasitic effects or surface current due to generation-recombination centers at the $\mathrm{Si}-\mathrm{SiO}_{2}$ interface. However the extracted density of interface traps in the Si bandgap, presented in Ch. 6 shows an increase of $D_{i t}$ as a function of X-ray dose in the midgap position both for n- and p-MOSFETs. A possible explanation is that the surface current contributes to the subthreshold current in the drain-source current measurement and influences the subthreshold swing of the curve. If this explanation is correct then the 
increase of $D_{i t}$ in the midgap position as a function of X-ray dose, is related to the higher contribution of surface current and not to a real effect as the interface traps are assumed to be amphoteric. In Figs. 9.9 (a) and (b) is shown the cross section and the biasing scheme for the drain-source current and the surface current measurement respectively.

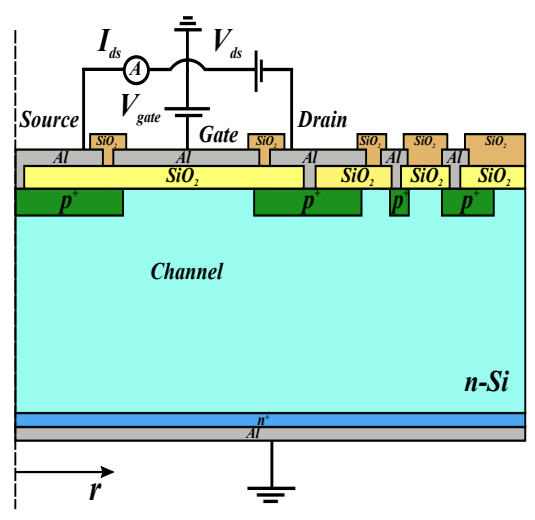

(a)

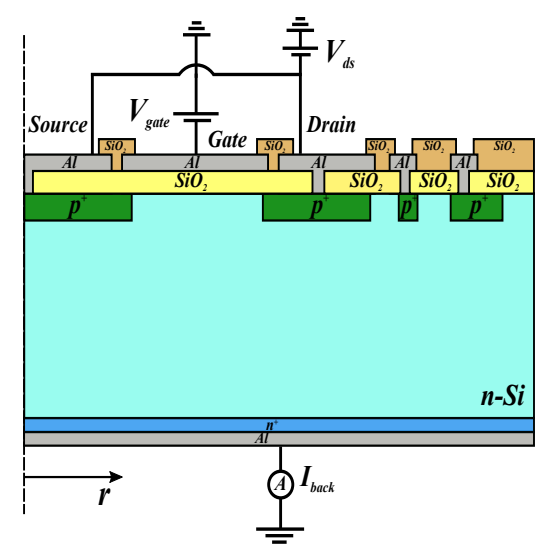

(b)

Figure 9.9: Cross section and biasing scheme of (a) drain-source current and (b) surface current measurement of the p-MOSFET $<111>$.

In Fig. 9.10 (a) is presented the $D_{i t}\left(\phi_{S}\right)$ as a function of X-ray dose of the p-MOSFET $<111>$ irradiated with $E_{\text {field }} \downarrow$, the $D_{i t}$ in the midgap position, $E_{I}$, decreases from $\approx 2.0 \cdot 10^{12} \mathrm{eV}^{-1} \mathrm{~cm}^{-2}$ to $\approx 6.0 \cdot 10^{11} \mathrm{eV}^{-1} \mathrm{~cm}^{-2}$ and for the last irradiation steps increases up to $\approx 3.5 \cdot 10^{12} \mathrm{eV}^{-1} \mathrm{~cm}^{-2}$.

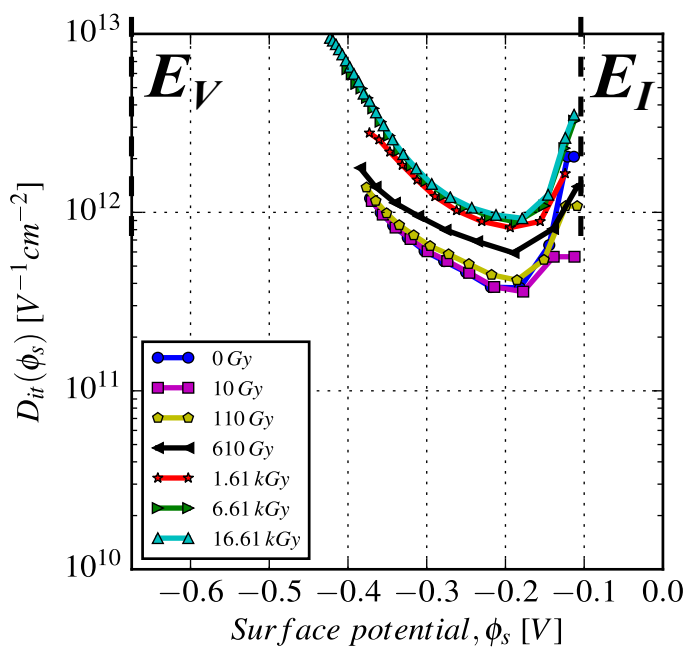

(a)

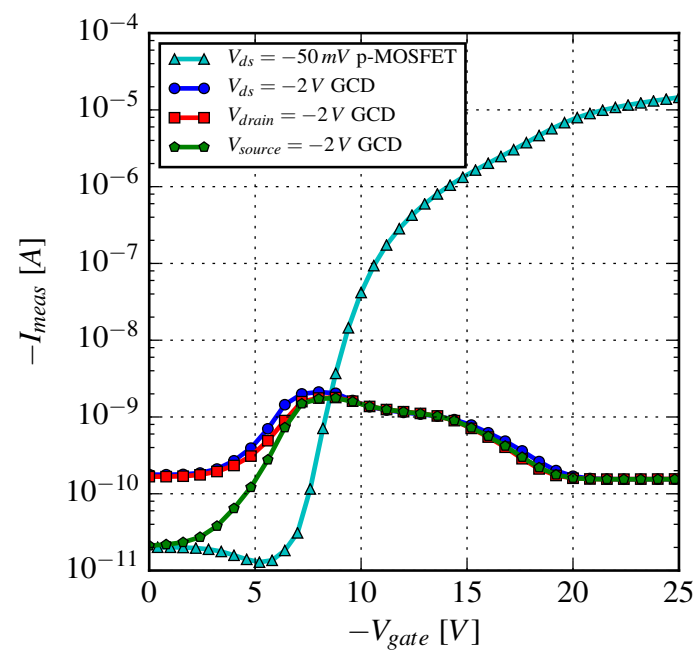

(b)

Figure 9.10: (a) $D_{i t}$ as a function of the surface potential before and after X-ray irradiation and, (b) Current measurement $I_{\text {meas }}$ as a function of $V_{\text {gate }}$ after $16.61 \mathrm{kGy} X$-ray dose of the p-MOSFET $<111>$ irradiated with $E_{\text {field }} \downarrow$. 
The explanation is that the surface current influences the subthreshold "stretchout" as shown in Fig. 9.10 (b) for the highest X-ray dose of $16.61 \mathrm{kGy}$. The surface current has been measured for the three possible bias configurations discussed in Ch. 7, reverse voltage $V_{d s}=-2 \mathrm{~V}$ on drain-source, reverse voltage $V_{\text {drain }}=-2 \mathrm{~V}$ on drain with source floating and reverse voltage $V_{\text {source }}=-2 \mathrm{~V}$ on source with drain floating. 



\section{Summary and conclusions}

In this thesis the surface radiation damage of the $\mathrm{Si}_{-} \mathrm{SiO}_{2}$ system on high-ohmic material has been investigated. The densities of the oxide charge $N_{o x}$, interface traps $N_{i t}$ and border traps at the $\mathrm{Si}_{-} \mathrm{SiO}_{2}$ interface have been determined as a function of X-ray dose. The study focused on the dependence of the above parameters on the electric field $E_{\text {field }}$ normal to the $\mathrm{Si}_{-} \mathrm{SiO}_{2}$ interface during irradiation and the time dependence after irradiation when the biasing conditions are changed. Irradiations were performed up to X-ray doses of $16.61 \mathrm{kGy}$ and for two field directions at the maximum $E_{\text {field }}$ according to TCAD simulations at the $\mathrm{Si}-\mathrm{SiO}_{2}$ interface in segmented silicon sensors under normal operation. In addition, annealing results of $N_{o x}, N_{i t}$ and border traps are presented after 256 hours annealing at $60{ }^{\circ} \mathrm{C}$.

The experimental techniques used to extract the $N_{o x}$ and $N_{i t}$ are standard techniques used to study ionizing radiation effects in electronics using $\mathrm{p}$ - and n-MOSFETs built on $\mathrm{n}$ - and $\mathrm{p}$-type $\mathrm{Si}$, respectively. Differences between silicon sensors and electronics are the lower doping density of the bulk material and the thicker oxide. Measurements were performed during and after irradiation, and parameter changes with long time constants were observed. The results show a significant field-direction dependence of the surface radiation damage parameters on high-ohmic material.

When the electric field direction during irradiation is pointing from the $\mathrm{Si}$ into the $\mathrm{SiO}_{2}$, for the n-type $\mathrm{Si}$, the $N_{o x}$ increases due to the increase of radiation-induced hole traps with increasing X-ray dose. However, for the p-type $\mathrm{Si}$, the $N_{o x}$ increases only up to $610 \mathrm{~Gy}$ and then decreases due to two electric field directions in the $\mathrm{SiO}_{2}$ (charge neutralization effect). When the electric field direction during irradiation is pointing from the $\mathrm{SiO}_{2}$ into the $\mathrm{Si}$, for the n-type $\mathrm{Si}$, the $N_{o x}$ increases up to $1.61 \mathrm{kGy}$ and then decreases. In a similar way for the p-type $\mathrm{Si}$, the $N_{o x}$ increases and decreases up to $6.61 \mathrm{kGy}$, depending on the field direction. In particular, for this case, the presence of two electric field components in the $\mathrm{SiO}_{2}$ was confirmed when a negative gate voltage was chosen during irradiation.

An increase of the $N_{i t}$ has been observed for all the conditions. However, MOSFETs are sensitive to the "active" interface traps which is only a fraction of interface traps in the Si bandgap. Assuming that the interface traps are acceptor-like in the upper part and donor-like in the lower part, for the n-type Si the "active" interface traps are donors below midgap and above the Fermi level. The acceptors above midgap are assumed to be neutral. For the p-type Si the "active" interface traps are acceptors above midgap and below the Fermi level and the donors below midgap are assumed to be neutral. The validity of the above assumption has been confirmed with the annealing results.

Charging and discharging of border traps has been measured by changing the $E_{\text {field }}$ direction after irradiation. The maximum effect has been found to be $25 \%$ of the oxide charge density after three hours biasing in the 
post-irradiation condition. Time constants in the order of 30 minutes have been observed for the charging and discharging, which confirms the near-interfacial nature of this kind of oxide traps.

The results show that surface radiation damage on high-ohmic material is qualitatively similar to radiation damage in electronics. In the past the Hamburg group has studied surface radiation damage up to 1 GGy $\mathrm{X}$-ray dose without electric field during irradiation using MOSCs and GCDs built on n-type Si. Now the investigated dose range was up to $16.61 \mathrm{kGy}$. It is found that for p-type Si irradiated with electric field pointing from the $\mathrm{SiO}_{2}$ into the $\mathrm{Si}$, the maximum oxide charge density already reached at a dose of $6.61 \mathrm{kGy}$ is approximately equal to the oxide charge density measured after irradiation of $1 \mathrm{GGy}$. The effects of $N_{o x}$, $N_{i t}$ and border traps have been separated for the two field directions during irradiation for n- and p-type Si. It is shown that surface radiation damage is more complicated than presently used in simulations, due to the X-ray dose and electric field dependence of $N_{o x}, N_{i t}$ and charging and discharging of the border traps. The results predict a spatial nonuniformity of the radiation-induced oxide charges at the $\mathrm{Si}_{-} \mathrm{SiO}_{2}$ interface in segmented silicon sensors due to the position-dependent electric field in the $\mathrm{SiO}_{2}$ and at the $\mathrm{Si}-\mathrm{SiO}_{2}$ interface. 


\section{Bibliography}

[1] The European X-Ray Free-Electron Laser (EuXFEL). URL:http: //www . xfel . eu/de/.

[2] J. Zhang, E. Fretwurst, H. Graafsma, R. Klanner, I. Kopsalis and J. Schwandt. Study of X-ray radiation damage in the AGIPD sensor for the European XFEL. Journal of Instrumentation, 9(5):C05022 arXiv:1312.3928v1, May 2014.

[3] M.M. Seibert et al. Single minivirus particles intercepted and imaged with an X-ray laser. Nature, 470:78-82, February 2011.

[4] J. Schwandt. Design of a Radiation Hard Pixel Sensor for X-ray Sciense. PhD thesis, University of Hamburg, DESY-THESIS-2014-029, 2014.

[5] AGIPD. URL:http://photon-science.desy.de/research/technical_groups/ detectors/projects/agipd/index_eng.html.

[6] Synopsis TCAD. URL:https://www. synopsys.com.

[7] J. Schwandt, E. Fretwurst, R. Klanner, I. Kopsalis, and J. Zhang. Design and first tests of a radiationhard pixel sensor for the European X-Ray Free-Electron Laser. IEEE Transactions on Nuclear Science, 61(4):1894-1901, August 2014.

[8] J. Zhang. X-ray radiation damage studies and design of a silicon pixel sensor for science at the XFEL. PhD thesis, University of Hamburg, DESY-THESIS-2013-018, 2013.

[9] SINTEF ICT. URL:https://www. sintef.no/.

[10] CMS Collaboration. Technical Proposal for the Phase-II Upgrade of the CMS Detector. URL:https://cds.cern.ch/record/2020886/files/LHCC-P-008.pdf.

[11] G. Steinbrück for the CMS Collaboration. The CMS silicon pixel detector for HL-LHC. Proceedings of Science, (Vertex 2016), September 2016.

[12] G. Steinbrïck for the CMS Collaboration. The upgrade of the CMS outer tracker. 2014 IEEE Nuclear Science Symposium and Medical Imaging Conference, November 2014.

[13] G. Steinbrück for the CMS Collaboration. Small pitch pixel sensors for the CMS Phase II upgrade. 2015 IEEE Nuclear Science Symposium and Medical Imaging Conference, October 2015.

[14] J. Erfle. Irradiation Study of Different Silicon Materials for the CMS Tracker Upgrade. PhD thesis, University of Hamburg, DESY-THESIS-2014-010, 2014. 
[15] M. Centis Vignali. Silicon Sensors for the Upgrades of the CMS Pixel Detector. $\mathrm{PhD}$ thesis, University of Hamburg, DESY-THESIS-2015-052, 2015.

[16] J. Schwandt, E. Fretwurst, E. Garutti, R. Klanner, I. Kopsalis. Surface effects in segmented silicon sensors. Nuclear Instruments and Methods in Physics Research A, doi.org/10.1016/j.nima.2016.06.032.

[17] J. Yorkston, A.C. Shotter, D.B. Syme, G. Huxtable. Interstrip surface effects in oxide passivated ionimplanted silicon strip detectors. Nuclear Instruments and Methods in Physics Research A, 262:353358, December 1987.

[18] A. Longoni, M. Sampietro, L. Strüder. Instability of the behaviour of high resistivity silicon detectors due to the presence of oxide charges. Nuclear Instruments and Methods in Physics Research A, 288(1):35-43, March 1990.

[19] C. Gössling, C. Lichau, M. Peters, J. Wüstenfeld and R. Wunstorf. Influence of surface damage on highly segmented silicon detectors. Il Nuovo Cimento A, 112(11):1369-1376, November 1999.

[20] J. Kalliopuska, S. Eränen, R. Orava. 3D simulations of 3D silicon radiation detector structures. Nuclear Instruments and Methods in Physics Research A, 568(1):27-33, November 2006.

[21] H. McDuff, M.R. Hoeferkamp, S. Seidel, R. Wang, C.J. Kenney, J. Hasi, S.I. Parker. The effect of humidity on reverse breakdown in $3 D$ silicon sensors. Nuclear Instruments and Methods in Physics Research A, 785:1-4, June 2015.

[22] T. Poehlsen, E. Fretwurst, R. Klanner, S. Schuwalow, J. Schwandt, J. Zhang. Charge losses in segmented silicon sensors at the $\mathrm{Si}_{-} \mathrm{SiO}_{2}$ interface. Nuclear Instruments and Methods in Physics Research A, 700:22-39, February 2013.

[23] T. Poehlsen, E. Fretwurst, R. Klanner, J. Schwandt, J. Zhang. Time dependence of charge losses at the $\mathrm{Si}_{-} \mathrm{SiO}_{2}$ interface in $p^{+} n$-silicon strip sensors. Nuclear Instruments and Methods in Physics Research A, 731:172-176, December 2013.

[24] T. Poehlsen. Charge Losses in Silicon Sensors and Electric-Field Studies at the $\mathrm{Si}_{-} \mathrm{SiO}_{2}$ Interface. $\mathrm{PhD}$ thesis, University of Hamburg, DESY-THESIS-2013-025, 2013.

[25] The Tracker Group of the CMS Collaboration. Impact of low-dose electron irradiation on $n^{+} p$ silicon strip sensors. Nuclear Instruments and Methods in Physics Research A, 803:100-112, August 2015.

[26] J. Zhang, I. Pintilie, E. Fretwurst, R. Klanner, H. Perrey and J. Schwandt. Study of radiation damage induced by $12 \mathrm{keV} \mathrm{X-rays} \mathrm{in} \mathrm{MOS} \mathrm{structures} \mathrm{built} \mathrm{on} \mathrm{high-resistivity} \mathrm{n-type} \mathrm{silicon.} \mathrm{Journal} \mathrm{of}$ Synchrotron Radiation, 19(3):340-346, May 2012.

[27] K. Ton That. Surface resistances in silicon sensors. BSc thesis, University of Hamburg, 2014.

[28] J.R. Brews. Physics of the MOS transistor. Silicon Integrated Circuits, Part A, Ed. D. Kahng, Applied Solid-State Science Series, Academic Press, New York, 1981.

[29] N. Arora. MOSFET modeling for VLSI simulation. World Scientific, Singapore, 1993. 
[30] E.H. Nicollian, J.R. Brews. MOS (Metal Oxide Semiconductor) Physics and Technology. John Wiley \& Sons, New Jersey, 2009.

[31] R. Lindner. Semiconductor surface varactor. The Bell System Technical Journal, XLI(3):803-831, May 1962.

[32] C.T. Sah. Characteristics of the Metal-Oxide-Semiconductor transistors. IEEE Transactions on Electron Devices, 11(7):324-345, July 1964.

[33] H.C. Pao and C.T. Sah. Effects of diffusion current on characteristics of metal-oxide (insulator)semiconductor transistors. Solid-State Electronics, 9(10):927-937, October 1966.

[34] C.T. Sah and H.C. Pao. The effects of fixed bulk charge on the characteristics of metal-oxidesemiconductor transistors. IEEE Transactions on Electron Devices, 13(4):393-409, April 1966.

[35] H.K.J. Ihantola and J.L. Moll. Design theory of a surface field-effect transistor. Solid-State Electronics, 7(6):423-430, June 1964.

[36] J.R. Brews. A charge-sheet model of the MOSFET. Solid-State Electronics, 21(2):345-355, February 1978.

[37] R.M. Swanson and J.M. Meindl. Ion-implanted complementary MOS transistors in low-voltage circuits. IEEE Journal of Solid-State Circuits, 7(2):146-153, April 1972.

[38] F. Van de Wiele. A long-channel MOSFET model. Solid-State Electronics, 22(12):991-997, December 1979 .

[39] M.B. Barron. Low level currents in insulated gate field effect transistors. Solid-State Electronics, 15(3):293-302, March 1972.

[40] R.F. Pierret and J.A. Shields. Simplified long-channel MOSFET theory. Solid-State Electronics, 26(2):143-147, February 1983.

[41] H. Katto and Y. Itoh. Analytical expressions for the static MOS transistor characteristics based on the gradual channel model. Solid-State Electronics, 17(12):1283-1292, December 1974.

[42] A. Nussbaum, R. Sinha, and D. Dokos. The theory of the long-channel MOSFET. Solid-State Electronics, 27(1):97-106, January 1984.

[43] S.C. Sun, J.D. Plummer. Electron mobility in inversion and accumulation layers on thermally oxidized silicon surfaces. IEEE Transactions on Electron Devices, 27(8):1497-1508, August 1980.

[44] S. Takagi, A. Toriumi, M. Iwase, and H. Tango. On the universality of inversion layer mobility in Si MOSFET's: Part I-effects of substrate impurity concentration. IEEE Transactions on Electron Devices, 41(12):2357-2362, December 1994.

[45] Y.C. Cheng and E.A. Sullivan. Relative importance of phonon scattering to carrier mobility in Si surface layer at room temperature. Journal of Applied Physics, 44(8):3619-3625, August 1973.

[46] Y.C. Cheng and E.A. Sullivan. Effect of Coulomb scattering on silicon surface mobility. Journal of Applied Physics, 45(1):187-192, January 1974. 
[47] Y.C. Cheng. On the scattering of electrons in magnetic and electric surface states by surface roughness. Surface Science, 27(3):663-666, October 1971.

[48] A. Hartstein, T.H. Ning and A.B. Fowler. Electron scattering in silicon inversion layers by oxide and surface roughness. Surface Science, 58(1):178-181, August 1976.

[49] Y.C. Cheng and E.A. Sullivan. On the role of scattering by surface roughness in silicon inversion layers. Surface Science, 34(3):717-731, February 1973.

[50] M.-S. Lin. A better understanding of the channel mobility of Si MOSFETs based on the physics of quantized subbands. IEEE Transactions on Electron Devices, 35(12):2406-2411, December 1988.

[51] D.K. Ferry. Effects of surface roughness in inversion layer transport. Electron Devices Meeting, 30:605-608, January 1984.

[52] T. Ohmi, K. Kotani, A. Teramoto, and M. Miyashita. Dependence of electron channel mobility on Si-SiO ${ }_{2}$ interface microroughness. IEEE Electron Device Letters, 12(12):652-654, December 1991.

[53] A.G. Sabnis and J.T. Clemens. Characterization of the electron mobility in the inverted $<100>S i$ surface. Electron Devices Meeting, 25(1):18-21, July 1979.

[54] Y. Tsividis. Operation and Modeling of The MOS Transistor. Oxford University Press, New York, 1998.

[55] D.K. Schroder. Semiconductor Material and Device Characterization. John Wiley \& Sons, New Jersey, 2006.

[56] F.H. De La Moneda, H.N. Kotecha, and M. Shatzkes. Measurement of MOSFET constants. IEEE Electron Device Letters, 3(1):10-12, January 1982.

[57] P.I. Suciu and R.L. Johnston. Experimental derivation of the source and drain resistance of MOS transistors. IEEE Transactions on Electron Devices, 27(9):1846-1848, September 1980.

[58] K.K. Ng and J.R. Brews. Measuring the effective channel length of MOSFETs. IEEE Circuits and Devices Magazine, 6(6):33-38, November 1990.

[59] S.S.-S. Chung and J.-S. Lee. A new approach to determine the drain-and-source series resistance of LDD MOSFET's. IEEE Transactions on Electron Devices, 40(9):1709-1711, September 1993.

[60] Y. Taur. MOSFET channel length: Extraction and interpretation. IEEE Transactions on Electron Devices, 47(1):160-170, January 2000.

[61] J.G.J. Chern, P.Chang, R.F. Motta, and N. Godinho. A new method to determine MOSFET channel length. IEEE Electron Device Letters, 1(9):170-173, September 1980.

[62] K. Terada and H. Muta. A new method to determine effective MOSFET channel length. Japanese Journal of Applied Physics, 18(5):953-959, May 1979.

[63] C. Hao, B. Cabon-Till, S. Cristoloveanu and G. Ghibaudo. Experimental determination of shortchannel MOSFET parameters. Solid-State Electronics, 28(10):1025-1030, October 1985. 
[64] F.B. McLean, T.R. Oldham. Basic mechanisms of radiation effects in electronic materials and devices. Technical Report US Army Lab Command, HDL-TR-2129, US Army Command, Harry Diamond Laboratories, MD, USA, 1987.

[65] R. D. Evans. The Atomic Nucleus. McGraw-Hill, New York, 1955.

[66] E. Segre. Nuclei and Particles. W. A. Benjamin, New York, 1965.

[67] V.A.J. van Lint, T.M. Flanagan, R.E. Leadon, J.A. Naber, and V.C. Rogers. Mechanisms of radiation effects in electronic materials, volume 1. Wiley-Interscience, New York, 1980.

[68] J. W. Corbett. Electron radiation damage in semiconductors and metals. Academic Press, New York, 1966.

[69] J.E. Gover. Basic radiation effects in electronics technology. 1984 IEEE NSREC Short Course, Colorado Springs, CO, July 1984.

[70] J.R. Srour. Basic mechanisms of radiation effects on electronic materials, devices, and integrated circuits. Technical Report Defense Nuclear Agency, DNA-TR-82-20, Defense Nuclear Agency, Northrop Corporation, California, USA, 1982.

[71] J.R. Schwank. Basic mechanisms of radiation effects in the natural space radiation environment. Technical Report US Department of Energy, SAND-94-1580C, US Department of Energy, Sandia National Laboratories, NM, USA, 1994.

[72] T.R. Oldham. Ionizing radiation effects in MOS oxides. World Scientific, Singapore, 1999.

[73] T.R. Oldham, and F.B. McLean. Total ionizing dose effects in MOS oxides and devices. IEEE Transactions on Nuclear Science, 50(3):483-499, June 2003.

[74] H.J. Barnaby. Total-ionizing-dose effects in modern CMOS technologies. IEEE Transactions on Nuclear Science, 53(6):3103-3121, December 2006.

[75] J.R. Schwank, M.R. Shaneyfelt, D.M. Fleetwood, J.A. Felix, P.E. Dodd, P. Pailet, and V. FerletCavrois. Radiation effects in MOS oxides. IEEE Transactions on Nuclear Science, 55(4):1833-1853, August 2008.

[76] H.L. Hughes and J.M. Benedetto. Radiation effects and hardening of MOS technology: Devices and circuits. IEEE Transactions on Nuclear Science, 50(3):500-521, June 2003.

[77] R.C. Alig and S. Bloom. Electron-hole-pair creation energies in semiconductors. Physical Review Letters, 35(22):1522-1525, December 1975.

[78] M. Moll. Radiation Damage in Silicon Particle Detectors Microscopic Defects and Macroscopic Properties. PhD thesis, University of Hamburg, DESY-THESIS-1999-040, 1999.

[79] National Institute of Standards and Technology. NIST SRD 124 Stopping-Power and Range Tables for Electrons, Protons, and Helium Ions. URL:http: / physics . nist.gov/PhysRefData/ Star/Text/ESTAR.html. 
[80] S. Franssila. Introduction to Microfabrication. John Wiley \& Sons, West Sussex, 2004.

[81] D.M. Fleetwood, S.T. Pantelides, R.D. Schrimpf. Defects in Microelectronic Materials and Devices. CRC Press, Taylor\& Francis Group, Florida, 2009.

[82] P.M. Lenahan and J.F. Conley. What can electron paramagnetic resonance tell us about the $\mathrm{Si}_{/} \mathrm{SiO}_{2}$ system? Journal of Vacuum Science \& Technology B, 16(4):2134-2153, July/August 1998.

[83] E.H. Poindexter and P.J. Caplan. Interface states and electron spin resonance centers in thermally oxidized (111) and (100) silicon wafers. Journal of Applied Physics, 52(2):879-884, February 1981.

[84] G.J. Gerardi, E.H. Poindexter, P.J. Caplan and N.M. Johnson. Interface traps and $P_{b}$ centers in oxidized (100) silicon wafers. Applied Physics Letters, 49(6):348-350, August 1986.

[85] D.M. Fleetwood. Long-term annealing study of midgap interface-trap charge neutrality. Applied Physics Letters, 60(23):2883-2885, June 1992.

[86] B.E. Deal. Standardized terminology for oxide charges associated with thermally oxidized silicon. IEEE Transactions on Electron Devices, 27(3):606-608, March 1980.

[87] D.M. Fleetwood. "Border traps" in MOS devices. IEEE Transactions on Nuclear Science, 39(2):269_ 271, April 1992.

[88] I.S. Esqueda. Modeling of Total Ionizing Dose Effects in Advanced Complementary Metal-OxideSemiconductor Technologies. PhD thesis, Arizona State University, 2011.

[89] P.S. Winokur, H.E. Boesch, J.M. McGarrity, and F.B. McLean. Field- and time-dependent radiation effects at the $\mathrm{SiO}_{2} / \mathrm{Si}$ interface of hardened MOS capacitors. IEEE Transactions on Nuclear Science, 24(6):2113-2118, December 1977.

[90] P.S. Winokur, F.B. McLean, H.E. Boesch. Physical processes associated with radiation-induced interface states. Technical Report US Army Lab Command, HDL-TR-2081, US Army Command, Harry Diamond Laboratories, MD, USA, 1986.

[91] N.S. Saks, D.B. Brown, and R.W. Rendell. Effects of switched gate bias on radiation-induced interface trap formation. IEEE Transactions on Nuclear Science, 38(6):1130-1139, December 1991.

[92] H.E. Boesch and F.B. McLean. Hole transport and trapping in field oxides. IEEE Transactions on Nuclear Science, 32(6):3940-3945, December 1985.

[93] F.B. McLean. A framework for understanding radiation-induced interface states in $\mathrm{SiO}_{2} \mathrm{MOS}$ structures. IEEE Transactions on Nuclear Science, 27(6):1651-1657, December 1980.

[94] D.A. Buchanan and D.J. DiMaria. Interface and bulk trap generation in metal-oxide-semiconductor capacitors. Journal of Applied Physics, 67(12):7439-7452, June 1990.

[95] M.R. Shaneyfelt, J.R. Schwank, D.M. Fleetwood, P.S. Winokur, K.L. Hughes, and F.W. Sexton. Field dependence of interface-trap buildup in polysilicon and metal gate MOS devices. IEEE Transactions on Nuclear Science, 37(6):1632-1640, December 1990. 
[96] P.M. Lenahan and P.V. Dressendorfer. Hole traps and trivalent silicon centers in metal/oxide/silicon devices. Journal of Applied Physics, 55(10):3495-3499, May 1984.

[97] D.B. Brown and N.S. Saks. Time dependence of radiation-induced interface trap formation in metaloxide-semiconductor devices as a function of oxide thickness and applied field. Journal of Applied Physics, 70(7):3734-3747, October 1991.

[98] A.J. Lelis, T.R. Oldham, H.E. Boesch, and F.B. McLean. The nature of the trapped hole annealing process. IEEE Transactions on Nuclear Science, 36(6):1808-1815, December 1989.

[99] P.V. Dressendorfer. Basic mechanisms for the millennium. Technical Report US Department of Energy, SAND-98-1388C, US Department of Energy, Sandia National Laboratories, NM, USA, 1998.

[100] D.M. Fleetwood. Radiation-induced charge neutralization and interface-trap buildup in metal-oxidesemiconductor devices. Journal of Applied Physics, 67(1):580-583, January 1990.

[101] D.M. Fleetwood, P.S. Winokur and L.C. Riewe. Predicting switched-bias response from steady-state irradiations. IEEE Transactions on Nuclear Science, 37(6):1806-1817, December 1990.

[102] P.S. Winokur, J.R. Schwank, P.J. McWhorter, P.V. Dressendorfer, and D.C Turpin. Correlating the radiation response of MOS capacitors and transistors. IEEE Transactions on Nuclear Science, 31(6):1453-1460, December 1984.

[103] P.J. McWhorter and P.S. Winokur. Simple technique for separating the effects of interface traps and trapped-oxide charge in metal-oxide-semiconductor transistors. Applied Physics Letters, 48(2):133135, January 1986.

[104] D.M. Fleetwood. Dual-transistor method to determine threshold-voltage shifts due to oxide-trapped charge and interface traps in metal-oxide-semiconductor devices. Applied Physics Letters, 55(5):466468, July 1989.

[105] C.M. Dozier, D.M. Fleetwood, D.B. Brown, and P.S. Winokur. An evaluation of low-energy X-ray and cobalt-60 irradiations of MOS transistors. IEEE Transactions on Nuclear Science, 34(6):1535-1539, December 1987.

[106] J.R. Schwank, P.S. Winokur, P.J. McWhorter, F.W. Sexton, P.V Dressendorfer, and D.C Turpin. Physical mechanisms contributing to device "rebound". IEEE Transactions on Nuclear Science, 31(6):1434-1438, December 1984.

[107] A.J. Lelis, H.E. Boesch, T.R. Oldham, and F.B. McLean. Reversibility of trapped hole annealing. IEEE Transactions on Nuclear Science, 35(6):1186-1191, December 1988.

[108] T.H. Ning. High-field capture of electrons by Coulomb-attractive centers in silicon dioxide. Journal of Applied Physics, 47(7):3203-3208, July 1976.

[109] A.H. Johnston. Super recovery of total dose damage in MOS devices. IEEE Transactions on Nuclear Science, 31(6):1427-1433, December 1984.

[110] R. Freeman and A. Holmes-Siedle. A simple model for predicting radiation effects in MOS devices. IEEE Transactions on Nuclear Science, 25(6):1216-1225, December 1978. 
[111] T.P. Ma, G. Scoggan, and R. Leone. Comparison of interface-state generation by 25-keV electron beam irradiation in p-type and n-type MOS capacitors. Applied Physics Letters, 27(2):61-63, July 1975.

[112] G.A. Scoggan and T.P. Ma. Effects of electron-beam radiation on MOS structures as influenced by the silicon dopant. Journal of Applied Physics, 48(1):294-300, January 1977.

[113] M. Knoll, D. Bräunig, and W.R. Fahrner. Generation of oxide charge and interface states by ionizing radiation and by tunnel injection experiments. IEEE Transactions on Nuclear Science, 29(6):14711478, December 1982.

[114] J.R. Schwank and W.R. Dawes. Irradiated silicon gate MOS device bias annealing. IEEE Transactions on Nuclear Science, 30(6):4100-4104, December 1983.

[115] P.V. Dressendorfer, J.M. Soden, J.J. Harrington, and T.V. Nordstrom. The effects of test conditions on MOS radiation-hardness results. IEEE Transactions on Nuclear Science, 28(6):4281-4287, December 1981.

[116] J. Zhang, E. Fretwurst, R. Klanner, J. Schwandt, J. Becker, I. Kopsalis, I. Pintilie and M. Turcato. $X$-ray induced radiation damage in segmented $p^{+} n$ silicon sensors. Proceeding of Science (Vertex 2012), (019), 2013.

[117] D. Brueske. Investigation of the field dependence of the injection of positive charges into the $\mathrm{SiO}_{2}$ at the Si-SiO 2 interface. BSc thesis, University of Hamburg, 2014.

[118] D. Brueske, E. Garutti, R. Klanner, I. Kopsalis, J. Schwandt, K. Ton That, J. Zhang. Investigation of the insulator layers for segmented silicon sensors before and after X-ray irradiation. 2014 IEEE Nuclear Science Symposium and Medical Imaging Conference, November 2014.

[119] J. Schwandt. Private communication.

[120] I. Kopsalis, R. Klanner and J. Schwandt. Measurement of the electric-field and time dependence of the effective oxide-charge density of the $\mathrm{Si}_{-} \mathrm{SiO}_{2}$ system. Journal of Instrumentation, 11(2):C02017, February 2016.

[121] R. Klanner, E. Fretwurst, I. Pintilie, J. Schwandt, J. Zhang. Study of high-dose X-ray radiation damage of silicon sensors. Nuclear Instruments and Methods in Physics Research A, 732:117-121, June 2013.

[122] G. Ghibaudo. An analytical model of conductance and transconductance for enhanced-mode MOSFETs. Phys. Stat. Sol. (a), 95(1):323-335, May 1986.

[123] G. Ghibaudo. New method for the extraction of MOSFET parameters. Electronics Letters, 24(9):543545, April 1988.

[124] A. Ortiz-Conde, F.J. García Sánchez, J.J. Liou, A. Cerdeira, M. Estrada, Y. Yue. A review of recent MOSFET threshold voltage extraction methods. Microelectronics Reliability, 42(4-5):583-596, AprilMay 2002. 
[125] L. Dobrescu, M. Petrov, D. Dobrescu, C. Ravariu. Threshold voltage extraction methods for MOS transistors. Semiconductor Conference, CAS 2000 Proceedings, 1:371-374, 10-14 October 2000.

[126] G. Ghibaudo. Analytical modeling of the MOS transistor. Physica Status Solidi (a), 113(1):223-240, May 1989.

[127] K. Rais. Surface roughness mobility model for silicon MOS transistors. Phys. Stat. Sol. (a), 146(2):853-858, May 1994.

[128] D.M. Fleetwood. Fast and slow border traps in MOS devices. IEEE Transactions on Nuclear Science, 43(3):779-786, June 1996.

[129] D.M. Fleetwood, M.R. Shaneyfelt, W.L. Warren, J.R. Schwank, T.L. Meisenheimer and P.S. Winokur. Border traps: Issues for MOS radiation response and long-term reliability. Microelectronics Reliability, 35(3):403-428, March 1995.

[130] D.M. Fleetwood, M.R. Shaneyfelt, L.C. Riewe, P.S. Winokur, and R.A. Reber. The role of border traps in MOS high-temperature postirradiation annealing response. IEEE Transactions on Nuclear Science, 40(6):1323-1334, December 1993.

[131] D.M. Fleetwood, M.R. Shaneyfelt, and J.R. Schwank. Estimating oxide-trap, interface-trap, and border-trap charge densities in metal-oxide-semiconductors transistors. Applied Physics Letters, 64(15):1965-1967, April 1994.

[132] D.M. Fleetwood, P.S. Winokur, R.A. Reber, T.L. Meisenheimer, J.R. Schwank, M.R. Shaneyfelt and L.C. Riewe. Effects of oxide traps, interface traps, and "border traps" on metal-oxide-semiconductor devices. Journal of Applied Physics, 73(10):5058-5074, May 1993.

[133] D.M. Fleetwood, W.L. Waren, J.R. Schwank, P.S. Winokur, M.R. Shaneyfelt, and L.C. Riewe. Effects of interface traps and border traps on MOS postirradiation annealing response. IEEE Transactions on Nuclear Science, 42(6):1698-1707, December 1995.

[134] D.M. Fleetwood, P.S. Winokur, L.C. Riewe, and R.A. Reber. Bulk oxide traps and border traps in metal-oxide-semiconductor capacitors. Journal of Applied Physics, 84(11):6141-6148, December 1998.

[135] R.K. Freitag, C.M. Dozier, and D.B. Brown. Growth and annealing of trapped holes and interface states using time-dependent biases. IEEE Transactions on Nuclear Science, 34(6):1172-1177, December 1987.

[136] V.S. Pershenkov, V.V. Belyakov, S.V. Cherepko, A.Y. Nikiforov, A.V. Sogoyan, V.N. Ulimov, and V.V. Emelianov. Effect of electron traps on reversibility of annealing. IEEE Transactions on Nuclear Science, 42(6):1750-1757, December 1995.

[137] D.M. Fleetwood, P.S. Winokur, and J.R. Schwank. Using laboratory X-ray and cobalt-60 irradiations to predict CMOS device response in strategic and space environments. IEEE Transactions on Nuclear Science, 35(6):1497-1505, December 1988. 
[138] D.M. Fleetwood, M.R. Shaneyfelt, J.R. Schwank, P.S. Winokur, and F.W. Sexton. Theory and application of dual-transistor charge separation analysis. IEEE Transactions on Nuclear Science, 36(6):1816-1824, December 1989.

[139] K.F. Galloway, M. Gaitan and T.J. Russell. A simple model for separating interface and oxide charge effects in MOS device characteristics. IEEE Transactions on Nuclear Science, 31:1497-1501, December 1984.

[140] D. Zupac, K.F. Galloway, R.D. Schrimpf, and P. Augier. Effects of radiation-induced oxide-trapped charge on inversion-layer hole mobility at 300 and 77 K. Journal of Applied Physics, 60(25):31563158, June 1992.

[141] D. Zupac, K.F. Galloway, P. Khosropour, S.R. Anderson, R.D. Schrimpf and P. Calvel. Separation of effects of oxide-trapped charge and interface-trapped charge on mobility in irradiated power MOSFETs. IEEE Transactions on Nuclear Science, 40(6):1307-1315, December 1993.

[142] F.B. McLean and H.E. Boesch. Time-dependent degradation of MOSFET channel mobility following pulsed irradiation. IEEE Transactions on Nuclear Science, 36(6):1772-1783, December 1989.

[143] A.P. Gnana Prakash, S.C. Ke and K. Siddappa. High-energy radiation effects on subthreshold characteristics, transconductance and mobility of n-channel MOSFETs. Semiconductor Science and Technology, 18(12):1037-1042, September 2003.

[144] A. Phanse, D. Sharma, A. Mallik, and J. Vasi. Carrier mobility degradation in metal-oxidesemiconductor field-effect transistors due to oxide charge. Journal of Applied Physics, 74(1):757759, July 1993.

[145] J.R. Brews. Surface potential fluctuations generated by interface charge inhomogeneities in MOS devices. Journal of Applied Physics, 43(5):2306-2313, May 1972.

[146] G. Declerck, R. Van Overstraeten, and G. Broux. Discussion of the surface-potential fluctuations caused by oxide-charge fluctuations. Journal of Applied Physics, 45(6):2593-2595, June 1974.

[147] R.J. Van Overstraeten, G. Declerck and G.L. Broux. The influence of surface potential fluctuations on the operation of the MOS transistor in weak inversion. IEEE Transactions on Electron Devices, 20(12):1154-1158, December 1973.

[148] R.J. Van Overstraeten, G. Declerck, and G.L. Broux. Inadequacy of the classical theory of the MOS transistor operating in weak inversion. IEEE Transactions on Electron Devices, 20(12):1150-1153, December 1973.

[149] Z. Shanfield. Thermally stimulated current measurements on irradiated MOS capacitors. IEEE Transactions on Nuclear Science, 30(6):4064-4070, December 1983.

[150] D.M. Fleetwood, R.A. Reber, L.C. Riewe, P.S. Winokur. Thermally stimulated current in $\mathrm{SiO}_{2}$. Microelectronics Reliability, 39(9):1323-1336, September 1999.

[151] R.A. Reber and D.M Fleetwood. Thermally stimulated current measurements of $\mathrm{SiO}_{2}$ defect density and energy in irradiated metal-oxide-semiconductor capacitors. Review of Scientific Instruments, 63(12):5714-5725, December 1992. 
[152] K. Lehovec, A. Slobodskoy, and J.L. Sprague. Field effect-capacitance analysis of surface states on silicon. Physica Status Solidi (b), 3(3):447-464, 1963.

[153] K. Lehovec. Frequency dependence of the impedance of distributed surface states in MOS structures. Applied Physics Letters, 8(2):48-50, January 1966.

[154] E.H. Nicollian, A. Goetzberger and A.D. Lopez. Expedient method of obtaining interface state properties from MIS conductance measurements. Solid-State Electronics, 12(12):937-944, December 1969.

[155] H. Deuling, E. Klausmann and A. Goetzberger. Interface states in $\mathrm{Si}_{-} \mathrm{SiO}_{2}$ interfaces. Solid-State Electronics, 15(5):559-571, May 1972.

[156] F.P. Heiman and G. Warfield. The effects of oxide traps on the MOS capacitance. IEEE Transactions on Electron Devices, 12(4):167-178, April 1965.

[157] M.A. Jupina and P.M. Lenahan. A spin dependent recombination study of radiation induced defects at and near the $\mathrm{Si}_{\mathrm{S}} \mathrm{O}_{2}$ interface. IEEE Transactions on Nuclear Science, 36(6):1800-1807, December 1989.

[158] W.L Warren, M.R. Shaneyfelt, D.M. Fleetwood, J.R. Schwank, P.S. Winokur, and R.A.B. Devine. Microscopic nature of border traps in MOS oxides. IEEE Transactions on Nuclear Science, 41(6):18171827, December 1994.

[159] W.L. Warren, D.M. Fleetwood, M.R. Shaneyfelt, and P.S. Winokur. Defect-defect hole transfer and the identity of border traps in $\mathrm{SiO}_{2}$ films. Physical Review B, 50(19):14710-14713, November 1994.

[160] G. Groeseneken, H.E. Maes, N. Beltran, and R.F. De Keersmaecker. A reliable approach to chargepumping measurements in MOS transistors. IEEE Transactions on Electron Devices, 31(1):42-53, January 1984.

[161] E.W. Enlow, R.L. Pease, D.R. Alexander. Subthreshold technique for fixed and interface trapped charge separation in irradiated MOSFETs. Technical Report Defense Nuclear Agency, DNA-TR-89157, Defense Nuclear Agency, Mission Research Corporation, NM, USA, 1990.

[162] M.R. Shaneyfelt, D.M. Fleetwood, P.S. Winokur, J.R. Schwank, and T.L. Meisenheimer. Effects of device scaling and geometry on MOS radiation hardness assurance. IEEE Transactions on Nuclear Science, 40(6):1678-1685, December 1993.

[163] F.W. Sexton and J.R. Schwank. Correlation of radiation effects in transistors and integrated circuits. IEEE Transactions on Nuclear Science, 32(6):3975-3981, December 1985.

[164] J.R. Brews. Subthreshold behavior of uniformly and nonuniformly doped long-channel MOSFET. IEEE Transactions on Electron Devices, 26(9):1282-1291, September 1979.

[165] J.R. Brews. Carrier-density fluctuations and the IGFET mobility near threshold. Journal of Applied Physics, 46(5):2193-2203, May 1975.

[166] J.R. Brews. Threshold shifts due to nonuniform doping profiles in surface channel MOSFET's. IEEE Transactions on Electron Devices, 26(11):1696-1710, November 1979. 
[167] Z. Shanfield, G.A. Brown, A.G. Revesz, and H.L. Hughes. A new MOS radiation-induced charge: Negative fixed interface charge. IEEE Transactions on Nuclear Science, 39(2):303-307, April 1992.

[168] P.J. McWhorter, P.S. Winokur, and R.A. Pastorek. Donor/acceptor nature of radiation-induced interface traps. IEEE Transactions on Nuclear Science, 35(6):1154-1159, December 1988.

[169] Z. Shanfield and M.M. Moriwaki. Critical evaluation of the midgap-voltage-shift method for determining oxide trapped charge in irradiated MOS devices. IEEE Transactions on Nuclear Science, 34(6):1159-1165, December 1987.

[170] L.M. Terman. An investigation of surface states at a silicon/silicon oxide interface employing metaloxide-silicon diodes. Solid-State Electronics, 5(5):285-299, September-October 1962.

[171] M. Gaitan, T.J. Russell. Measurement of radiation-induced interface traps using MOSFETs. IEEE Transactions on Nuclear Science, 31(6):1256-1260, December 1984.

[172] K.F. Galloway, C.L. Wilson, and L.C. Witte. Charge-sheet model fitting to extract radiation-induced oxide and interface charge. IEEE Transactions on Nuclear Science, 32(6):4461-4465, December 1985.

[173] H.E. Boesch and T.L. Taylor. Charge and interface state generation in field oxides. IEEE Transactions on Nuclear Science, 31(6):1273-1279, December 1984.

[174] H.E. Boesch. Interface-state generation in thick $\mathrm{SiO}_{2}$ layers. IEEE Transactions on Nuclear Science, 29(6):1445-1451, December 1982.

[175] H.E. Boesch and J.M. McGarrity. Charge yield and dose effects in MOS capacitors at $80 \mathrm{~K}$. IEEE Transactions on Nuclear Science, 23(6):1520-1525, December 1976.

[176] P.S. Winokur and H.E. Boesch. Interface-state generation in radiation-hard oxides. IEEE Transactions on Nuclear Science, 27(6):1647-1650, December 1980.

[177] T.P. Ma. Interface trap transformation in radiation or hot-electron damaged MOS structures. Semiconductor Science and Technology, 4(12):1061-1079, December 1989.

[178] T.P. Chen, D.S.H. Chan and W.K. Chim. Determination of substrate doping, substrate carrier lifetime and density of surface recombination centres of MOSFETs by gate-controlled-diode measurements. Semiconductor Science and Technology, 11:672-678, August 1996.

[179] C. Becker, C. Gößling, C. Lichau, T. Wübben, J. Wüstenfeld, R. Wunstorf. Gate-controlled diodes for characterization of the $\mathrm{Si}_{-} \mathrm{SiO}_{2}$ interface with respect to surface effects of silicon detectors. Nuclear Instruments and Methods in Physics Research A, 444(3):605-613, April 2000.

[180] A.S. Grove and D.J. Fitzgerald. Surface effects on p-n junctions: Characteristics of surface spacecharge regions under non-equilibrium conditions. Solid-State Electronics, 9(8):783-806, August 1966.

[181] A. Grove. Physics and Technology of Semiconductor Devices. John Wiley \& Sons, New York, 1967. 
[182] W. Shockley and W.T. Read. Statistics of the recombinations of holes and electrons. Physical Review, 87(5):835-842, September 1952.

[183] C.T. Sah, R.N. Noyce and W. Shockley. Carrier generation and recombination in p-n junctions and p-n junction characteristics. Proc. Inst. Radio Engrs., 45(9):1228-1243, September 1957.

[184] J. Cai and C.T. Sah. Evidence of discrete interface traps on thermally grown thin silicon oxide films. Applied Physics Letters, 74(2):257-259, January 1999.

[185] R.F. Pierret. The gate-controlled diode $s_{0}$ measurement and steady-state lateral current flow in deeply depleted MOS structures. Solid-State Electronics, 17(12):1257-1269, December 1974.

[186] T.P. Ma and P.V. Dressendorfer. Ionizing radiation effects in MOS devices and circuits. John Wiley \& Sons, New York, 1989.

[187] P.J. McWhorter, D.M. Fleetwood, R.A. Pastorek and G.T. Zimmerman. Comparison of MOS capacitor and transistor postirradiation response. IEEE Transactions on Nuclear Science, 36(6):1792-1799, December 1989.

[188] M.R. Shaneyfelt, J.R. Schwank, D.M. Fleetwood and P.S. Winokur. Effects of irradiation temperature on MOS radiation response. IEEE Transactions on Nuclear Science, 45(3):1372-1378, June 1998.

[189] Y. Roh, L. Trombetta, and D.J. DiMaria. Interface traps induced by hole trapping in metal-oxide semiconductor devices. Journal of Non-Crystalline Solids, 187:165-169, July 1995.

[190] T.R. Oldham, A.J. Lelis, and F.B. McLean. Spatial dependence of trapped holes determined from tunneling analysis and measured annealing. IEEE Transactions on Nuclear Science, 33(6):12031209, December 1986.

[191] D.M. Fleetwood, R.A. Reber, P.S. Winokur. Trapped-hole annealing and electron trapping in metaloxide-semiconductor devices. Applied Physics Letters, 60(16):2008-2010, April 1992.

[192] P.J. McWhorter, S.L. Miller, and W.M. Miller. Modeling the anneal of radiation-induced trapped holes in a varying thermal environment. IEEE Transactions on Nuclear Science, 37(6):1682-1689, December 1990.

[193] S. Scharf, M. Schmidt and D. Bräunig. Temperature-dependent positive oxide charge annealing by electron tunneling. Semiconductor Science and Technology, 10(5):586-591, December 1995.

[194] A.J. Lelis, T.R. Oldham, and W.M. DeLancey. Response of interface traps during high-temperature anneals. IEEE Transactions on Nuclear Science, 38(6):1590-1597, December 1991.

[195] N.S. Saks and M.G. Ancona. Generation of interface states by ionizing radiation at 80K measured by charge pumping and subthreshold slope techniques. IEEE Transactions on Nuclear Science, 34(6):1348-1354, December 1987.

[196] J.M. Benedetto and H.E. Boesch. MOSFET and MOS capacitor responses to ionizing radiation. IEEE Transactions on Nuclear Science, 31(6):1461-1466, December 1984. 
[197] J.R. Schwank, D.M. Fleetwood, M.R. Shaneyfelt, and P.S. Winokur. A critical comparison of chargepumping, dual-transistor, and midgap measurement techniques. IEEE Transactions on Nuclear Science, 40(6):1666-1677, December 1993.

[198] S.M. Sze and K.K. Ng. Physics of Semiconductor Devices. John Wiley \& Sons, New Jersey, 2007. 


\section{List of publications}

\section{First author}

2016 Measurement of the electric-field and time dependence of the effective oxide-charge density of the $\mathrm{Si}_{-} \mathrm{SiO}_{2}$ system Journal of Instrumentation 11 C02017 (2016)

\section{Contributing author}

2016 Surface effects in segmented silicon sensors

Nuclear Instruments and Methods in Physics Research A, doi:10.1016/j.nima.2016.06.032

2016 The influence of edge effects on the determination of the doping profile of silicon pad diodes

arXiv: $1605.00778 \mathrm{v} 1$

2014 Investigation of the insulator layers for segmented silicon sensors before and after X-ray irradiation (conference paper)

2014 IEEE Nuclear Science Symposium and Medical Imaging Conference

2014 Design and first tests of a radiation-hard pixel sensor for the European X-Ray Free-Electron Laser

IEEE Transactions on Nuclear Science Vol.61 (4) 1894-1901

2014 Study of X-ray radiation damage in the AGIPD sensor for the European XFEL

Journal of Instrumentation 9 C05022 (2014)

arXiv:1312.3928v1 



\section{Acknowledgements}

First of all, I would like to thank Prof. Dr. Erika Garutti and Prof. Dr. Robert Klanner for giving me the opportunity of joining the Detector Lab and supervising this PhD Thesis. Cordial thanks for your support and the helpful ideas on this interesting and difficult topic of ionizing radiation effects on silicon sensors. Also, I would like to thank Prof. Dr. Heinz Graafsma and Dr. Georg Steinbrück for agreeing to referee my $\mathrm{PhD}$ Thesis and Prof. Dr. Günter Sigl for chairing the disputation. Many thanks to Dr. Eckhart Fretwurst for the useful discussions on various topics on silicon sensors. In addition, I would like to thank my close colleagues, Dr. Joern Schwandt and Dr. Jiaguo Zhang, for the excellent collaboration on measurements, irradiations and TCAD simulations. I am sincerely thankful to the German Academic Exchange Service, Deutscher Akademischer Austauschdienst "DAAD", for funding my PhD fellowship for three years at the University of Hamburg. Thanks to the Bachelor students that we worked together, Dominik Brüske, Khai Ton That and Martin Weberpals for their measurements and the critical questions that I received from them. Also, I would like to thank the technical group of the Detector Lab, Michael Matysek, Peter Buhmann, Wolfgang Gärtner and Jörg Wellhausen for maintaining the measurement infrastructure in an excellent shape. Many thanks to the secretary of the Lab, Gundula Serbser for her support on my applications for fellowship extension every year. Furthermore, many thanks to the IT support on Linux systems from Rainer Peter Feller and Ronald Mohrmann. Thank you very much to all the students of the Detector Lab for the table soccer games after lunch. Of course, I would like to thank my friends for the nice moments in the Hamburg metropolitan area. Finally, I would like to thank my family for their support during my PhD studies. 



\section{Declaration of Authorship}

I, IOANNIS KOPSALIS, declare that this thesis titled, "Surface effects in segmented silicon sensors" and the work presented in it are my own. I confirm that:

- This work was done mainly while in candidature for my $\mathrm{PhD}$ degree at the University of Hamburg.

- Where any part of this thesis has previously been submitted for a degree or any other qualification at this University or any other institution, this has been clearly stated.

- Where I have consulted the published work of others, this is always clearly attributed.

- Where I have quoted from the work of others, the source is always given. With the exception of such quotations, this thesis is entirely my own work.

- I have acknowledged all main sources of help.

- Where the thesis is based on work done by myself jointly with others, I have made clear exactly what was done by others and what I have contributed myself.

Signed: Ioannis Kopsalis

Place: Hamburg, Germany

Date: 05.01.2017 
